\title{
Search for Supersymmetry in Final States with Leptons with the ATLAS Detector at the Large Hadron Collider
}

Dissertation

zur Erlangung des mathematisch-naturwissenschaftlichen Doktorgrades

"Doctor rerum naturalium"

der Georg-August-Universität Göttingen

im Promotionsprogramm ProPhys

der Georg-August University School of Science (GAUSS)

vorgelegt von

Matthias Hamer

aus Paderborn

Göttingen, 2013 
1) Dr. Carsten Hensel, Elementarteilchenphysik/LHC, II. Physikalisches Institut

2) Prof. Dr. Arnulf Quadt, Elementarteilchenphysik/LHC, II. Physikalisches Institut

3) Prof. Dr. Wolfram Kollatschny, Astrophysik, Institut für Astrophysik

Mitglieder der Prüfungskommission

Referent: $\quad$ Prof. Dr. Arnulf Quadt, Elementarteilchenphysik/LHC, II. Physikalisches Institut

Korreferent: Dr. Carsten Hensel, Elementarteilchenphysik/LHC, II. Physikalisches Institut

2. Korreferent: Prof. Dr. Klaus Desch, Elementarteilchenphysik/LHC,ILC, Universität Bonn

Weitere Mitglieder der Prüfungskommission:

1) Prof. Dr. Wolfram Kollatschny, Astrophysik, Institut für Astrophysik

2) Priv. Doz. Dr. Kevin Kröninger, Elementarteilchenphysik/LHC, II. Physikalisches Institut

3) Prof. Dr. Ariane Frey, Elementarteilchenphysik/KEKB/ILC, II. Physikalisches Institut

4) Priv. Doz. Dr. Jörn Große-Knetter, Elementarteilchenphysik/Detektorentwicklung, II. Physikalisches Institut

Tag der mündlichen Prüfung: 10.05.2013 


\section{Search for Supersymmetry in Final States with Leptons with the ATLAS Detector at the Large Hadron Collider}

Dissertation

zur Erlangung des mathematisch-naturwissenschaftlichen Doktorgrades

"Doctor rerum naturalium"

der Georg-August-Universität Göttingen

im Promotionsprogramm ProPhys

der Georg-August University School of Science (GAUSS)

vorgelegt von

Matthias Hamer

aus Paderborn

Göttingen, 2013

II.Physik-UniGö-Diss-2013/02 



\title{
Search for Supersymmetry in Final States with Leptons with the ATLAS Detector at the Large Hadron Collider
}

\author{
by \\ Matthias Hamer
}

A study of the CMSSM, where a phenomenologically motivated measure for finetuning is introduced, is presented to motivate SUSY searches in final states with leptons at the LHC. For these searches a study of ATLAS lepton triggers is performed, and a technique for the application of trigger efficiencies in data analyses dealing with MC simulations is described. Particular emphasis is put on a new method for the propagation of uncertainties on the sum of event weights. Finally, a search for RPC SUSY in final states with four or more leptons is presented, where no significant excess above the SM expectation is found. Limits are set in two simplified models for the production of a $\tilde{\chi}_{2}^{0}$ in association with a $\tilde{\chi}_{3}^{0}$. Assuming a well defined SUSY mass hierarchy, a Bino-like neutralino LSP with a mass of up to 250 $\mathrm{GeV}$ is excluded at the $95 \%$ confidence level. 



\section{Contents}

1 Introduction 1

2 The Standard Model of Elementary Particle Physics and the Minimal Supersymmetric Standard Model $\quad 5$

2.1 The Standard Model of Elementary Particle Physics . . . . . . . . . . . . . . . 5

2.1.1 Local Gauge Invariance, Interactions and the Higgs Mechanism . . . . . . 6

2.1.2 Particle Content . . . . . . . . . . . . . . . . . . . . . 9 9

2.1.3 Renormalisation and the Running of Parameters . . . . . . . . . . . . 10

2.1.4 Scattering Amplitudes at Hadron Colliders . . . . . . . . . . . . . . . . . 11

2.1.5 Unanswered Questions and Fundamental Problems . . . . . . . . . . . . . 12

2.2 Extensions of the Standard Model . . . . . . . . . . . . . . . . . . . 13

2.3 The Minimal Supersymmetric Standard Model . . . . . . . . . . . . . . . . 15

2.3 .1 Supersymmetry . . . . . . . . . . . . . . . . . 15

2.3.2 The Minimal Supersymmetric Standard Model . . . . . . . . . . . . . . 18

2.3.3 Supersymmetry Breaking . . . . . . . . . . . . . . . . . . . 20

2.3.4 The Phenomenology of the MSSM . . . . . . . . . . . . . . 23

2.3.5 Simplified SUSY Breaking Models . . . . . . . . . . . . . . . 28

2.3 .6 Fine-Tuning . . . . . . . . . . . . . . . . . . . . . . . . 30

3 The Large Hadron Collider and the ATLAS Experiment 33

3.1 The Large Hadron Collider at CERN . . . . . . . . . . . . . . . . . . . . . 33

3.1.1 Design and Experiments . . . . . . . . . . . . . . . . 33

3.1 .2 Operation in $2010-2012 \ldots \ldots \ldots \ldots \ldots \ldots$

3.2 The ATLAS Experiment . . . . . . . . . . . . . . . . . 37

3.2.1 The ATLAS Coordinate System . . . . . . . . . . . . . . 37

3.2 .2 The ATLAS Inner Detector . . . . . . . . . . . . . . . . . . 38

3.2 .3 The ATLAS Calorimeters . . . . . . . . . . . . . . . . . . . . 39

3.2.4 The ATLAS Muon Systems . . . . . . . . . . . . . . . . . . . . . 41

3.2.5 The ATLAS Magnet Systems . . . . . . . . . . . . . . . . . . . 42

3.2 .6 The ATLAS Trigger System . . . . . . . . . . . . . . . . . . . . 43

3.2 .7 Performance of the ATLAS detector . . . . . . . . . . . . . 46

4 The CMSSM and NUHM1 After Two Years of LHC Operation 49

4.1 Global Fits of SUSY Models . . . . . . . . . . . . . . . . . . . . . . . . 49

4.1 .1 The Idea . . . . . . . . . . . . . . . . . . . . . . . . . . . . . . . 49

4.1 .2 The Fittino Framework . . . . . . . . . . . . . . . 50

4.1 .3 Observables . . . . . . . . . . . . . . . . . . 53

4.2 The CMSSM/mSUGRA-Model after Two Years of LHC data . . . . . . . . . 58

$4.2 .1 \quad$ Fit Results . . . . . . . . . . . . . . . . . . . 58

4.2 .2 Finetuning . . . . . . . . . . . . . . . . . . . . . . . 59

4.3 Summary of Results . . . . . . . . . . . . . . . . . . . . 66 
5 Measurements and Application of ATLAS Lepton Trigger Efficiencies $\quad 69$

5.1 Leptonic Final States at the LHC . . . . . . . . . . . . . . . . . . . 69

5.1.1 Final States with Leptons in the Standard Model . . . . . . . . . . . . . . 70

5.1.2 Searches for Supersymmetry in Leptonic Final States . . . . . . . . . 70

5.2 Trigger Efficiencies and Statistics . . . . . . . . . . . . . . . 71

5.2 .1 Definition of a Trigger Efficiency . . . . . . . . . . . . . 71

5.2 .2 Trigger Matching . . . . . . . . . . . . . . . . . . . 73

5.2 .3 Interpretation of Measurements of Trigger Efficiencies . . . . . . . . . . . . 74

5.3 Application of Trigger Efficiencies in Data Analyses . . . . . . . . . . . . . 76

5.3.1 Scale Factors and Event Weights . . . . . . . . . . . . . . 76

5.3 .2 Calculation of Event Weights . . . . . . . . . . . . . . . 78

5.3.3 Calculation and Propagation of Uncertainties on Event Weights . . . . . . 81

5.3.4 Sanity Checks for Trigger Reweighting . . . . . . . . . . . . . . . . . . 84

5.4 Performance of the ATLAS Muon Triggers during the $\sqrt{s}=7 \mathrm{TeV}$ Run $\ldots . . .101$

5.4.1 Techniques for Measuring Muon Trigger Efficiencies . . . . . . . . . . . . 101

5.4.2 Performance of the Primary ATLAS Muon Triggers during the $\sqrt{s}=7 \mathrm{TeV}$

Run in $2011 \ldots \ldots$. . . . . . . . . . . . . . . . 103

6 Search for R-Parity Conserving SUSY in Final States with Four Leptons 109

6.1 Simplified Models for $\tilde{\chi}_{2}^{0}-\tilde{\chi}_{3}^{0}$ Production . . . . . . . . . . . . . . 109

6.1 .1 The pMSSM as a Motivation . . . . . . . . . . . . . . 110

6.1 .2 The Simplified Model $v 2 A \ldots \ldots \ldots$. . . . . . . . . . . 120

6.1.3 The Simplified Model v2B . . . . . . . . . . . . . . . . . . . . . 125

6.2 Analysis Overview . . . . . . . . . . . . . . . . . 126

6.3 Object Reconstruction and Event Preselection . . . . . . . . . . . . . . . 127

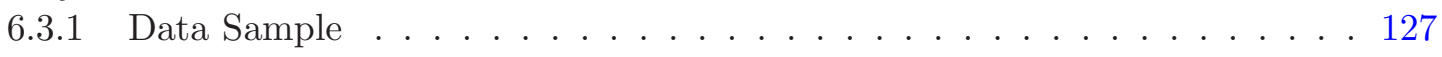

6.3 .2 Trigger . . . . . . . . . . . . . . . . . . . . . . . 128

6.3 .3 Cleaning Cuts . . . . . . . . . . . . . . . . . . . . . 128

6.3 .4 Object Reconstruction . . . . . . . . . . . . . . . . . . . . 129

6.4 MC samples . . . . . . . . . . . . . . . . . . . . . . . . . . . . . 132

$6.4 .1 \mathrm{SM} \mathrm{MC} \mathrm{.} \mathrm{.} \mathrm{.} \mathrm{.} \mathrm{.} \mathrm{.} \mathrm{.} \mathrm{.} \mathrm{.} \mathrm{.} \mathrm{.} \mathrm{.} \mathrm{.} \mathrm{.} \mathrm{.} \mathrm{.} \mathrm{.} \mathrm{.} \mathrm{.} \mathrm{.} 132$

6.4 .2 Signal MC . . . . . . . . . . . . . . . . . . . . . 133

6.4.3 Corrections to the $\mathrm{MC} \ldots \ldots \ldots \ldots \ldots$

6.5 Signal Region . . . . . . . . . . . . . . . . . . . . 133

6.6 Estimation of the SM Background . . . . . . . . . . . . . . . 135

6.6.1 Irreducible Background Processes . . . . . . . . . . . . . . . . . . 135

6.6.2 Reducible Background Processes . . . . . . . . . . . . . . . . 137

6.6.3 Background Expectations from MC . . . . . . . . . . . . . . . 138

6.6.4 Estimation of the Reducible Background . . . . . . . . . . . . . . . . 139

6.6.5 Validation of the Background Modelling . . . . . . . . . . . . . . 144

6.7 Analysis Results . . . . . . . . . . . . . . . . . . . . . 148

6.7.1 Statistical Interpretation of the Results . . . . . . . . . . . . . . 149

7 Discussion and Outlook $\quad 153$

7.1 Summary and Discussion . . . . . . . . . . . . . . 153

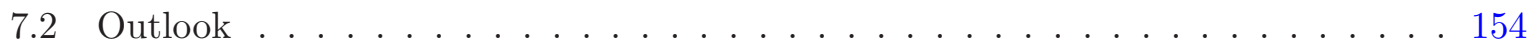

$\begin{array}{lr}\text { Bibliography } & 156\end{array}$ 
Acknowledgements 175 



\section{Introduction}

More than a century ago, it was believed that physicists had solved the last great mysteries of nature [1]. The remaining caveats, which could not be explained at that time, like the spectrum of the black-body radiation, were thought of as minor inconsistencies to be resolved very soon. However, the approaches that were followed for the resolution of the remaining problems brought up the formulation of quantum theory and general relativity, messing up the whole view of the world as accepted in those days and pushing open the door to new scientific territories, which were unknown to exist until then. Now, more than a hundred years after most questions in physics seemed to be answered and the theoretical description of nature was assumed to be complete, we have to admit that the number of unanswered, fundamental questions has steadily increased since then.

One of the fields in physics that arose from a number of new experimental results and new theoretical concepts is the field of elementary particle physics, whose birthday is sometimes defined by the discovery of the electron by J. J. Thompson in 1897 [2]. In the following decades, discoveries of more and more elementary particles and bound states were announced, finally opening out in the discovery of what is today known as the top quark [3, 4] and the tau neutrino [5]. The current theory of particle physics, the Standard Model, which has been developed for many years, has until now been most successfull in describing the experimental results.

However, it is known today that the Standard Model is not the full story. There is a number of shortcomings, which require either an extended or a completely new theory. It is one of the goals of the experiments at the Large Hadron Collider (LHC) at the European Organization for Nuclear Research (CERN) near Geneva, Switzerland, to perform searches for new physics and thus provide experimental input for the construction of such an extended or new theory.

The LHC has been running smoothly for nearly three years by now, and with a steadily increasing instantaneous luminosity and an increase in the centre-of-mass energy up to $8 \mathrm{TeV}$, physicists all over the earth have started to investigate an energy regime that has not been studied under laboratory conditions before, making their first steps in the task to "explore strange new worlds, to seek out new particles and new interactions, to boldly go where no man has gone before".

A milestone was already achieved in the summer of 2012, when two of the major experiments at the LHC announced the discovery of a new boson in the search for the Standard Model Higgs boson at a mass of $126 \mathrm{GeV}$, roughly [6, 7]. While it is yet to be revealed if this is truly the Standard Model Higgs boson, the quest for physics beyond the Standard Model continues.

One family of the extensions to the SM, which resolve some of its shortcomings with bravery, are supersymmetric extensions, the best studied of which is the Minimal Supersymmetric Standard Model. This theory predicts a number of so far undiscovered particles, the so called superpartners of the SM particles, which makes it attractive not only for particle physics but also for cosmology. This is because the lightest of these new particles might turn out to constitute a significant amount of the cold dark matter in the universe, the nature of which remains unknown until now.

The question if supersymmetry is realized in nature is therefore one of the most interesting unanswered questions in modern particle physics. Although a number of experiments at the 
Large Electron Positron Collider and at the Tevatron, as well as astrophysical experiments, have excluded certain regions in the supersymmetric parameter space, the experiments at the LHC can put even more powerful constraints on supersymmetric models.

While early searches at the LHC have not shown any evidence for supersymmetry, there is still a large variety of supersymmetric models than can possibly be discovered by the experiments analysing the collision data. In particular if measurements that have been peformed in the pre-LHC era are taken into account, searches for supersymmetry with leptons in the final state seem interesting. Such events provide a clear signature via the leptons, and can efficiently be selected for further analysis by the experiments using lepton triggers. As in the Standard Model the inclusive lepton production cross section at the LHC decreases with an increasing lepton multiplicity, searches for supersymmetry in events with a large number of leptons in the final state can possibly provide clear evidence for new physics.

Studies for searches for supersymmetry with two and more leptons in the final state are presented in this thesis, which is organised as follows: In chapter 2, a short review of the Standard Model of elementary particle physics is given, followed by a roundup of supersymmetry in general and the minimal supersymmetric standard model. The LHC and the ATLAS experiment are briefly described in chapter 3 , followed by a global analysis of two highly constrained supersymmetric models in chapter 4 . In chapter 5 , a method of applying lepton trigger efficiencies in data analyses is described, where emphasis is put on a newly developed method for the calculation of uncertainties on the sum of event weights, respecting event-by-event correlation coefficients. Finally, in chapter 6, a search for supersymmetry in events with four or more leptons with the ATLAS experiment at the LHC is presented, where limits are set in two simplified models for the associate production of two neutralinos. 
Conventions Whenever it is feasible, natural units are used throughout this thesis, i.e. $c=$ $\hbar=1$. Three-vectors are written in bold symbols, while normal size symbols are used to indicate scalars or four-vectors, for which sometimes also Greek indices are added. The contravariant space-time coordinates are given by

$$
x^{\mu}=(t, \mathbf{x}),
$$

the contravariant four-momentum is given by

$$
p^{\mu}=(E, \mathbf{p}) .
$$

The space-time metric $g_{\mu \nu}$ is the Minkowski metric with $g_{00}=1$. The $\gamma$ matrices and the Pauli spin matrices are used as defined in [8]. Context dependent the Pauli matrices are denoted by the symbols $\sigma$ or $\tau$.

SM vector fields are described by latin letters with a relativistic index $\mu$ or $\nu$, Dirac fermions are usually described by the Greek letter $\psi$, Weyl fermion fields by the Greek letters $\xi, \lambda$ and $\chi$, and scalar fields are usually described by the Greek letter $\phi$. For the fermions, the notation as used in [8] is followed closely. An important convention is, that a Dirac spinor is written as an object consisting of two Weyl spinors:

$$
\psi_{D}=\left(\begin{array}{c}
\chi_{\alpha} \\
\xi^{\dagger \dot{\alpha}}
\end{array}\right) \quad \text { with } \alpha=1,2 \text { and } \dot{\alpha}=1,2,
$$

where dotted indices are used for right chiral spinors, which are the hermitian conjugates of left chiral spinors, such that

$$
\chi_{\dot{\alpha}}^{\dagger}=\left(\chi_{\alpha}\right)^{\dagger}=\left(\chi^{\dagger}\right)_{\dot{\alpha}} .
$$

If useful, the chirality of a Weyl fermion is indicated by indices $L$ or $R$. Usually sums over family indicies $i$ and gauge group indices $a$ are implied. Whenever it is feasible, such and similar indices are suppressed. In the Feynman diagrams, the horizontal axis is usually the time-axis. 



\section{The Standard Model of Elementary Particle Physics and the Minimal Supersymmetric Standard Model}

The Standard Model of Elementary Particle Physics (SM) describes the dynamics and interactions of particles at what is today believed to be the most fundamental level. While it has a long history of remarkable successes in explaining and predicting observations $[9,10,11,12]$, as of today it is known to be an effective theory with a number of shortcomings and it has to be extended to become a more complete theory of nature. As symmetries of various types have played a special role in the development of the SM, it seems natural to extend the existing theory by imposing new non-trivial external symmetries, which without spoiling the consistency of the theory inevitably leads to Supersymmetry (SUSY).

The main features of the SM and the Minimal Supersymmetric Standard Model (MSSM) are explained in this chapter, with a focus on the concepts that are important for the following chapters. After a brief review of the SM and its shortcomings, some candidates for an extension are outlined, followed by a more detailed charactersisation of SUSY and the Minimal Supersymmetric Standard Model.

It is the goal of this chapter to set the theoretical framework for the study that is presented in this thesis. For that matter only a brief overview of the most essential features is provided here, and the given description of the SM and the MSSM is neither complete nor self-contained. More information on each topic can be found in the literature. The overview of the Standard Model in this chapter is heavily based on $[13,14,15,16,17]$. The description of Supersymmetry and the Minimal Supersymmetric Standard Model are mainly taken from $[8,15,18]$.

\subsection{The Standard Model of Elementary Particle Physics}

The SM is a locally gauge invariant, renormalisable, Lorentz covariant quantum field theory. On a very fundamental level, it distinguishes between two types of elementary particles, which are fermions with half-integer spin and bosons with integer spin. All known, visible matter in the universe is made of fermions, while vector bosons mediate forces between the matter particles. Both bosons and fermions acquire inert mass through their coupling to the only known scalar field in the SM, the Higgs field.

While it is today believed that there are four fundamental interactions between particles, the SM can only give a description of three of these. Electromagnetic interactions take place between particles that carry an electric charge, while the strong force affects all particles carrying a colour charge. All known left chiral fermions interact via the weak force. There is no description of gravity in the SM, which in contrast to the interactions that are described by it in some theories is mediated by a tensor boson. The strength of all interactions is characterised by a coupling constant, which at relatively low scales $(\leq 1 \mathrm{GeV})$ is at the order of 1 for strong interactions, 0.7 for weak interactions, and $10^{-2}$ for electromagnetic interactions. Despite being called coupling constants, these numbers vary as a function of the relevant energy scale. At scales accessible by 
experiments today, gravity is much weaker than any of the other three interactions, and it is therefore neglected in calculations.

\subsubsection{Local Gauge Invariance, Interactions and the Higgs Mechanism}

The equations of motion for a system with an arbitrary collection of fields $\left\{\Psi_{i}\right\}$ is usually derived from the action integral

$$
S\left[\Psi_{i}\right]=\int \mathcal{L}\left(\Psi_{i}, \partial_{\mu} \Psi_{i}, x^{\mu}\right) d^{4} x,
$$

where $x^{\mu}$ are the relativistic space-time coordinates and $\mathcal{L}$ is the Lagrangian density, by using Hamilton's principle

$$
\delta S\left[\Psi_{i}\right]=0,
$$

for the physical field configurations. This yields the Euler-Lagrange equations,

$$
\partial_{\mu}\left(\frac{\partial \mathcal{L}}{\partial\left(\partial_{\mu} \Psi_{i)}\right.}\right)-\frac{\partial \mathcal{L}}{\partial \Psi_{i}}=0
$$

A physical system is said to be invariant under a transformation $\mathcal{T}$, if the action integral (2.1) does not change under $\mathcal{T}$, which implies that $\mathcal{L}$ may only change by a total derivative. $\mathcal{T}$ is then called a symmetry transformation. It was shown by Emmy Noether that continous symmetries of physical systems are directly related to conserved quantities [19]. For a continous symmetry transformation,

$$
\begin{aligned}
& x^{\mu} \rightarrow x^{\mu}+\delta x^{\mu}=x^{\mu}+\varepsilon X^{\mu}, \\
& \Psi_{i} \rightarrow \Psi_{i}+\delta \Psi_{i}=\Psi_{i}+\varepsilon^{\prime} \Xi_{i},
\end{aligned}
$$

Noether's theorem states that the continuity equation

$$
\partial_{\mu} j^{\mu}=0,
$$

holds for the current

$$
j^{\mu}=-\frac{\partial \mathcal{L}}{\partial\left(\partial_{\mu} \Psi_{i}\right)} \Xi_{i}+\left(\frac{\partial \mathcal{L}}{\partial\left(\partial_{\mu} \Psi_{i}\right)} \partial_{\nu} \Psi_{i}-\mathcal{L} \delta_{\mu}^{\nu}\right) X^{\nu} .
$$

The implication of a conserved quantity $Q=\int j^{0} d^{3} x$ is one of the most remarkable consequences of a symmetry of a physical system.

On quantum states, such transformations are performed by operators which are representations of Lie groups. The elements of the Lie groups depend on one or more continuous parameters and are differentiable with respect to these. Wigner showed that symmetry transformations on quantum states must be performed by either unitary or anti-unitary operators [20]. Using the generators of the Lie group, $\mathbf{A}$, any unitary operator $U$ can then be written as a function of the group parameters $\Theta$ :

$$
\begin{array}{r}
U(\boldsymbol{\Theta})=e^{i \boldsymbol{\Theta} \cdot \mathbf{A}}, \\
U(d \boldsymbol{\Theta})=1+i d \boldsymbol{\Theta} \cdot \mathbf{A} .
\end{array}
$$

$U$ being unitary requires the generators $\mathbf{A}$ to be hermitian, which means that the generators of symmetry transformations may correspond to observables. In general, the group parameters 
can depend on the space-time coordinates, $\Theta=\Theta(x)$. If (not) so, the physical system is said to be locally (globally) invariant under the symmetry transformations $U$. For a consistent physical quantum field theory it is mandatory to impose local gauge invariance.

Invariance under local phase transformations can only be achieved by introducing a covariant derivative and therefore extending the field content of the Lagrangian, if it contains at least one possible state with a non-zero eigenvalue to the generators of that symmetry transformations. The procedure introduces interaction terms in the Lagrangian of an a-priori free theory and is another example for the unique importance of symmetries in the SM. Corresponding to three of the known interactions, the SM Lagrangian is required to be invariant under local $S U(3)_{C} \times$ $S U(2)_{L} \times U(1)_{Y}$ phase transformations.

The strong interaction, that is described by the $S U(3)_{C}$ symmetry, is mediated by eight linearly independent gluons. Demanding the free Lagrangian for an arbitrary number of massless fermion fields $\left\{\psi_{i}\right\}$ to be invariant under local $S U(3)_{C}$ phase transformations forces the introduction of a colour octet $G_{\mu}^{a}$ in the covariant derivative and requires a kinetic term for it, such that with the coupling constant $g_{s}$

$$
\mathcal{L}_{S U(3)_{C}}=\underbrace{\mathcal{L}_{\text {fermion }}^{\text {free }}}_{=i \overline{\psi_{i}} \gamma^{\mu} \partial_{\mu} \psi_{i}}-g_{s}\left(\overline{\psi_{i}} \gamma^{\mu} T_{a} \psi_{i}\right) G_{\mu}^{a}-\frac{1}{4} G_{\mu \nu}^{a} G_{a}^{\mu \nu},
$$

where the $T_{a}$ are the generators of the $S U(3)_{C}$. For an infinitesimal phase transformation $U=1+\alpha_{a} T_{a}$, the gluon fields are required to transform as

$$
G_{\mu}^{a} \rightarrow G_{\mu}^{a}-\frac{1}{g_{s}} \partial_{\mu} \alpha_{a}(x)-f_{a b c} \alpha_{b} G_{\mu}^{c},
$$

with the $S U(3)$ structure constants $f_{a b c}$. The strong field strength tensor is given by

$$
G_{\mu \nu}^{a}=\partial_{\mu} G_{\nu}^{a}-\partial_{\nu} G_{\mu}^{a}-g_{s} f_{a b c} G_{\mu}^{b} G_{\nu}^{c} .
$$

Imposing invariance under local $S U(2)_{L}$ gauge transformations requires the addition of an $S U(2)_{L}$ triplet in the covariant derivative and the addition of a kinetic term for it in the Lagrangian. Early experiments have shown the $V-A$ structure of the weak interaction, which means that the vector fields introduced by the $S U(2)_{L}$ symmetry do only couple to fermion states with negative chirality. The chirality of a Dirac fermion is obtained by applying the operator

$$
\gamma^{5}=i \gamma^{0} \gamma^{1} \gamma^{2} \gamma^{3}
$$

to the state. An eigenstate with the eigenvalue $+1(-1)$ is called a right (left) chiral fermion. The structure constants of the $S U(2)_{L}$ are closely related to the asymmetric symbol $\varepsilon_{i j k}$, such that the $S U(2)_{L}$ field strength tensor can be written as

$$
\mathbf{W}_{\mu \nu}=\partial_{\mu} \mathbf{W}_{\nu}-\partial_{\nu} \mathbf{W}_{\mu}-g \mathbf{W}_{\mu} \times \mathbf{W}_{\nu},
$$

where $g$ is a weak coupling constant. The Lagrangian for a system of massless fermion fields after imposing an $S U(3)_{C} \times S U(2)_{L}$ symmetry is given by

$$
\mathcal{L}_{S U(3)_{C} \times S U(2)_{L}}=\mathcal{L}_{S U(3)_{C}}-\frac{g}{2} \bar{\chi}_{i L} \gamma^{\mu} \boldsymbol{\tau} \mathbf{W}_{\mu} \chi_{i L}-\frac{1}{4} \mathbf{W}_{\mu \nu} \mathbf{W}^{\mu \nu},
$$

in which the weak isospin eigenvalue $\frac{1}{2}$ for the known left chiral fermion fields has been inserted and $\boldsymbol{\tau}$ are the Pauli spin matrices. There are no couplings between the $\mathbf{W}$ bosons and fermion states with positive chirality. 
Finally, by demanding invariance under local $U(1)_{Y}$ phase transformations, the vector field $B_{\mu}$ is introduced, which yields the Lagrangian

$$
\mathcal{L}_{S U(3)_{C} \times S U(2)_{L} \times U(1)_{Y}}=\mathcal{L}_{S U(3)_{C} \times S U(2)_{L}}-\bar{\psi}_{i} \gamma^{\mu} \frac{g^{\prime} Y}{2} B_{\mu} \psi_{i}-\frac{1}{4} B_{\mu \nu} B^{\mu \nu},
$$

with $B_{\mu \nu}=\partial_{\mu} B_{\nu}-\partial_{\nu} B_{\mu}$ and the coupling constant $g^{\prime}$. The introduced fields $\mathbf{W}_{\mu}$ and $B_{\mu}$ represent the bosons in their gauge eigenstates. In the SM these mix according to the weak mixing angle $\theta_{W}$ to give the physical mass eigenstates,

$$
\begin{aligned}
A_{\mu}^{0} & =\cos \theta_{W} B_{\mu}+\sin \theta_{W} W_{\mu}^{0} \\
Z_{\mu} & =-\sin \theta_{W} B_{\mu}+\cos \theta_{W} W_{\mu}^{0} \\
W_{\mu}^{ \pm} & =\frac{1}{\sqrt{2}}\left(W_{\mu}^{1} \pm W_{\mu}^{2}\right)
\end{aligned}
$$

The interaction terms in the Lagrangian do not only describe the coupling of the fermions to the gauge bosons, but since both the $S U(3)$ as well as the $S U(2)$ groups have non-vanishing structure constants, triple and quadruple couplings of the gluons as well as the electroweak bosons are introduced.

Nearly all known fermions do have a non-vanishing rest mass, as do three of the twelve gauge bosons known in the SM. Mass terms for fermions and vector bosons

$$
\mathcal{L}_{\text {mass }}^{\text {fermion }}=-m \bar{\psi} \psi \quad \text { and } \mathcal{L}_{\text {mass }}^{\text {boson }}=-\frac{1}{2} m_{B}^{2} B_{\mu} B^{\mu}
$$

would spoil the gauge invariance of the theory and are therefore forbidden. Thus the massive particles must acquire their masses in a different, gauge invariant way. In the SM, this is achieved via the Higgs mechanism [21, 22, 23, 24].

An additional complex scalar $S U(2)_{L}$ doublet $\phi$ with weak hypercharge $Y=1$ is added to the theory with a potential $V(\phi)$. The Lagrangian for this part is given by

$$
\begin{aligned}
\mathcal{L}_{\text {Higgs }} & =D_{\mu} \phi^{\dagger} D^{\mu} \phi-V\left(\phi, \phi^{\dagger}\right) \\
& =\left|\left(\partial_{\mu}-i g \mathbf{T} \cdot \mathbf{W}_{\mu}-i g^{\prime} \frac{Y}{2} B_{\mu}\right) \phi\right|^{2}-V\left(\phi, \phi^{\dagger}\right)
\end{aligned}
$$

with $V\left(\phi, \phi^{\dagger}\right)=\frac{1}{2} \mu^{2} \phi^{\dagger} \phi+\frac{1}{4} \lambda\left(\phi^{\dagger} \phi\right)^{2}$. Depending on the choice of $\mu$ and $\lambda$, the minimum of the potential and with it the natural choice for the field variables for perturbative calculations in the Lagrangian, is not equal to $\phi=0$. If $\mu^{2}<0$ and $\lambda>0$, the minimum of the Higgs potential is given by the vacuum expectation value $v$,

$$
\phi^{\dagger} \phi=-\frac{\mu^{2}}{\lambda}=v^{2} .
$$

If one of the states satisfying (2.19) is chosen as the ground state, it spontaneously breaks local gauge invariance by adding a constant term to one of the components of the scalar field. Apart from one massive scalar boson (the Higgs boson), due to Goldstone's theorem, this gives rise to three massless scalar bosons (Goldstone bosons, see [25, 26] and also [27]). The degrees of freedom of these can be absorbed by the gauge fields introduced by the local symmetry, which by this mechanism acquire a mass term. By spontaneously breaking the local $S U(2)_{L}$ gauge invariance in the SM, three of the intermediate vector bosons get a mass. Also, trilinear as well as quadrilinear couplings between the Higgs boson and the massive vector bosons are introduced. 
In the SM, all fermion masses are generated by the same complex scalar $S U(2)_{L}$ doublet by adding gauge invariant terms of the form

$$
\mathcal{L}_{\text {Higgs }}^{\text {Fermion }}=-G_{f}\left(\bar{\chi}_{i L} \phi \xi_{i R}+\bar{\xi}_{i R} \phi^{*} \chi_{i L}\right) .
$$

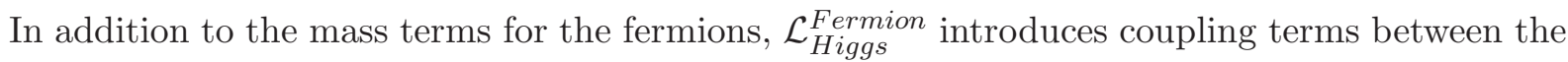
fermions and the Higgs boson. The strength of each of these couplings is proportional to the respective fermion mass. The full Lagrangian of the SM is then given by

$$
\mathcal{L}_{S M}=\mathcal{L}_{S U(3)_{C} \times S U(2)_{L} \times U(1)_{Y}}+\mathcal{L}_{\text {Higgs }}+\mathcal{L}_{\text {Higgs }}^{\text {Fermion }} .
$$

\subsubsection{Particle Content}

The fermion fields of the SM are summarised in Table 2.1. These fermions are usually divided into two different groups, the quarks, which carry a colour charge, and the leptons, which do not. Leptons, for which as for quarks there are six different mass eigenstates, can be observed as quasi-free particles in nature, while quarks almost exclusively appear as constituents of bound states (hadrons); due to confinement any observable state in the SM must not carry any net colour charge, such that baryons (consisting of three quarks or three anti-quarks) and mesons (one quark and one anti-quark) are the only bound states of quarks that have been observed so far. The SM does also allow bound states with more constituents - pentaquarks, for instance but the experimental evidence for such states is controversial [28, 29, 30, 31, 32, 33]. There are no known bound states with top-quarks because their average lifetime is much smaller than the hadronisation time. Each quark eigenstate can carry one of three different types of colour charges $(\mathrm{R}, \mathrm{G}, \mathrm{B})$ and does therefore form an $S U(3)_{C}$ triplet. Quarks carry either one or two thirds of the electric charge of an electron or positron. Concerning the electroweak interactions, the gauge eigenstates of the quarks are different from the mass eigenstates. The gauge eigenstates for $T_{3}=-\frac{1}{2}$ mix to the mass eigenstates according to the $C K M$-Matrix,

$$
\left(\begin{array}{c}
d^{\prime} \\
s^{\prime} \\
b^{\prime}
\end{array}\right)=\left(\begin{array}{lll}
V_{u d} & V_{u s} & V_{u b} \\
V_{c d} & V_{c s} & V_{c b} \\
V_{t d} & V_{t s} & V_{t b}
\end{array}\right)\left(\begin{array}{l}
d \\
s \\
b
\end{array}\right) .
$$

Left chiral quarks can be combined to $S U(2)_{L}$ doublets, while right handed quarks form $S U(2)_{L}$ singlets.

While they do not couple strongly, concerning the electroweak interactions leptons are very similar to quarks. Although the details are not yet fully understood, also for neutrinos the gauge eigenstates are different from the mass eigenstates and a mixing similar to equation (2.22) appears (PMNS-Matrix). However, due to their small masses neutrinos are usually considered to be massless in the SM and the $S U(2)_{L}$ doublets are usually defined as in Table 2.3. This also implies that there are neither right chiral neutrinos nor left chiral anti-neutrinos. In contrast to quarks, leptons carry integer electric charge and do not carry any net colour charge. Therefore leptons are observed as quasi-free particles as well as in bound states with hadrons, for instance.

Leptons are assigned a lepton number, $L$, which is +1 for leptons and -1 for anti-leptons. Quarks are assigned a baryon number, $B$, which is $+\frac{1}{3}$ for quarks and $-\frac{1}{3}$ for anti-quarks. In the SM, both $L$ and $B$ are conserved.

The vector boson fields of the SM are listed in Table 2.2. As described above, only the $W$ bosons and the $Z$ boson acquire a mass due to their coupling to the Higgs field. Both the photon and the gluon have a rest mass of 0 and possess therefore only two linearly independent polarisation states. The most relevant quantum numbers of all gauge fields and the fermion fields are listed in Table 2.3. 


\begin{tabular}{|rllll|}
\hline Particle & Type & Generation & Mass $[\mathrm{MeV}]$ & El. Charge \\
\hline electron-neutrino $\left(\nu_{e}\right)$ & lepton & I & $\geq 0$ & 0 \\
electron $(\mathrm{e})$ & lepton & I & 0.511 & -1 \\
\hline muon-neutrino $\left(\nu_{\mu}\right)$ & lepton & II & $\geq 0$ & 0 \\
muon $(\mu)$ & lepton & II & 105.7 & -1 \\
\hline tau-neutrino $\left(\nu_{\tau}\right)$ & lepton & III & $\geq 0$ & 0 \\
tau $(\tau)$ & lepton & III & $1,776.82 \pm 0.16$ & -1 \\
\hline up $(\mathrm{u})$ & quark & I & $1.8 \leq m_{u} \leq 3.0$ & $+2 / 3$ \\
down $(\mathrm{d})$ & quark & I & $4.5 \leq m_{d} \leq 5.5$ & $-1 / 3$ \\
\hline charm $(\mathrm{c})$ & quark & II & $1,275_{-25}^{+25}$ & $+2 / 3$ \\
strange $(\mathrm{s})$ & quark & II & $95_{-5}^{+5}$ & $-1 / 3$ \\
\hline top (t) & quark & III & $(173.5 \pm 1.0) \cdot 10^{3}$ & $+2 / 3$ \\
bottom $(\mathrm{b})$ & quark & III & $\left(4.18_{-0.03}^{+0.03}\right) \cdot 10^{3}$ & $-1 / 3$ \\
\hline & & & &
\end{tabular}

Table 2.1: The SM fermions with their masses and electric charge. Leptons and quarks are separated into three generations, as illustrated here. The masses and charges are taken from [34]. The extraordinary role of the top quark in particle physics becomes clear, as its mass is nearly two orders of magnitude above the mass of the b-quark, which is the next heaviest fermion. Due to its high mass, the top quark decays before it can build any bound states. For the top quark, the mass from direct measurements is quoted, while the value of the b-quark mass in the $\overline{\mathrm{MS}}$ scheme is quoted. Small uncertainties have been omitted in the table.

\begin{tabular}{|rlll|}
\hline Particle & Associated Interaction & Mass $[\mathrm{GeV}]$ & El. Charge \\
\hline gluon $(\mathrm{g})$ & strong & 0 & 0 \\
photon $(\gamma)$ & electromagnetic & 0 & 0 \\
$W^{ \pm}$ & weak & $80.385 \pm 0.015$ & \pm 1 \\
$Z$ & weak & $91.188 \pm 0.002$ & 0 \\
\hline
\end{tabular}

Table 2.2: The Standard Model gauge bosons with their masses and electric charge. The numbers are taken from [34]. In addition to their masses and charge, the associated gauge interactions are shown.

\subsubsection{Renormalisation and the Running of Parameters}

The SM is an effective theory that is valid only up to a certain energy scale, the cut-off scale $\Lambda_{C}$. Nevertheless, calculations in the SM do necessarily include contributions from above that energy scale, such that the results of calculations can become infinite. In order to restore the physical meaning of the theory, the infinities arising in the calculations are absorbed in a consistent way in the parameters of the SM, such as the particle masses and coupling constants. The process introduces radiative corrections to the parameters, which this way become a function of the energy scale and are normalised to the actual scale the calculation is carried out at. If a finite number of parameters is sufficient to restore the predictive power of the theory by removing all infinities, a theory is called renormalisable.

For the SM, which is a renormalisable theory, the dependence of a parameter $\alpha$ on the energy scale $Q$ is described by beta functions,

$$
\frac{\partial \alpha}{\partial Q}=\frac{1}{Q} \beta_{n}(\alpha)
$$

where $n$ is the maximum number of loops included in the diagrams that are used to calculate 


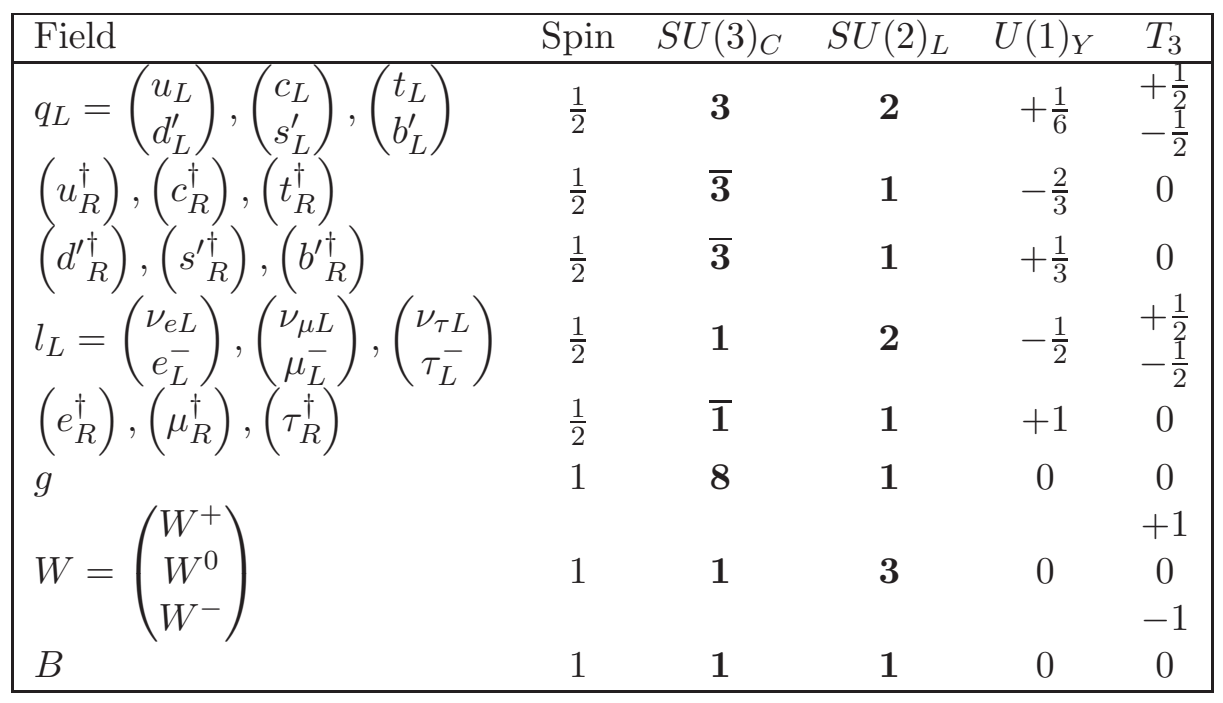

Table 2.3: The Standard Model fields and their spin, their $S U(3)_{C}$ and $S U(2)_{L}$ representations, the weak hypercharge and the third component of the weak isospin. The Higgs field is not included in this table.

the radiative corrections to the parameters.

It is by loop corrections that an observable quantity such as the physical mass of a particle can depend on nearly all parameters of the theory. For instance, the observable mass of the Higgs boson does not only depend on the parameter $\mu$ in the Higgs potential but by loop corrections it is heavily correlated with the mass of the top quark, for instance. The loop corrections do as well affect observables such as branching fractions, decay widths and coupling strengths, but depending on the mass scale of the particles included in the loops, they can become very small, such that most accurate measurements are needed to assess the nature of all loops included in a certain process.

\subsubsection{Scattering Amplitudes at Hadron Colliders}

In the parton model, hadrons are described as bound states consisting of quarks and gluons. In each hadron there are two or three valence quarks, that define the quantum numbers of the bound state, as well as a sea of gluons and quark-antiquark pairs. The sea is produced by soft QCD interactions within the hadron. The total momentum of a hadron is then carried by its constituents according to so called parton distribution functions (PDFs). Given the total longitudinal momentum $P$ of a hadron $\mathrm{H}$, the probability for finding a quark, antiquark or gluon $i$ with longitudinal momentum $x P$ in that hadron is given by the PDFs $f_{i}^{H}\left(x, q^{2}\right)$. The argument $q^{2}$ is the transferred momentum squared in the partonic interaction, and the dependence of the PDFs on $q^{2}$ are described by the so-called DGLAP equations. The PDFs for one hadron are constrained by the requirement

$$
\sum_{i} \int_{0}^{1} x f_{i}^{H}\left(x, q^{2}\right) d x=1,
$$

where the sum includes separately all quarks, all anti-quarks and the gluon. Various groups provide fits of the proton PDFs to data from deep inelastic scattering experiments for instance $[35,36]$. These PDFs can be used via the factorisation theorem to calculate cross-sections 
for processes at hadron colliders, such as the LHC. For the process $p p \rightarrow F X$, where $F$ is some interesting final state and $X$ are the proton remnants, the total cross-section can be calculated to

$$
\sigma(p p \rightarrow F X)=\sum_{i, j} \int d x_{1} d x_{2} f_{i}^{1}\left(x, \mu^{2}\right) f_{j}^{2}\left(x, \mu^{2}\right) \hat{\sigma}_{i j \rightarrow F}\left(x_{1} P_{1}, x_{2} P_{2}, \alpha_{s}\left(\mu^{2}\right), \frac{q^{2}}{\mu^{2}}\right),
$$

with the proton momenta $P_{1}$ and $P_{2}$, the partonic cross-section $\hat{\sigma}_{i j \rightarrow F}$ and the factorisation scale $\mu$, that is used to separate between the perturbative region and the non-perturbative region.

If $F$ contains any coloured particles, such as quarks or gluons in the SM, these particles will hadronise very quickly, i.e. they will form colourless bound states. This is usually described by phenomenologically motivated models that are tuned towards data from experiments due to the impossibility to perform perturbative calculations in the relevant energy regime.

Scattering amplitudes for processes including new physics can be calculated using equation (2.25) by using the appropriate non-SM Feynman rules. Usually the mass scale of such models is assumed to be large, such that the SM parton distributions functions are used.

\subsubsection{Unanswered Questions and Fundamental Problems}

Despite its unmatched success in explaining and predicting a huge number of measurements and observations in the field of high energy physics during the last decades, the SM is an effective theory and does not provide a complete description of nature. There is a number of both aesthetical as well as physical problems with the SM.

The loop corrections to the mass of the Higgs boson are quadratically divergent in the cut-off scale,

$$
\delta m_{H}^{2} \propto \Lambda_{C}^{2}
$$

Electroweak precision data (by loop corrections) favours a Higgs mass at the order of $100 \mathrm{GeV}[9,10,11]$, and the new particle discovered by the experiments at the Large Hadron Collider (LHC) in 2012, which is widely suspected to be the Higgs boson, has a mass of roughly $126 \mathrm{GeV}[6,7]$. If the SM is assumed to be a valid theory up to the Planck scale, $M_{P} \sim 10^{19} \mathrm{GeV}$, enormous cancellations must appear in the loop corrections in order to obtain a physical mass of $126 \mathrm{GeV}$. This is usually considered to be unnatural and is referred to as fine-tuning, or the hierarchy problem of the SM.

Another aesthetical flaw of the SM is the evolution of the gauge couplings

$$
\alpha_{1}=\frac{5 g^{\prime 2}}{12 \pi} \quad \alpha_{2}=\frac{g^{2}}{4 \pi} \quad \alpha_{3}=\frac{g_{s}^{2}}{4 \pi} .
$$

If the evolution according to the SM is carried out up to the Planck scale, the gauge couplings do not unify. In analogy to the unification of the electromagnetic and the weak interaction at the electroweak scale $m_{Z}$, a unification of all three interactions at some energy scale - that is typically assumed to be very large, $M_{G U T}>10^{15} \mathrm{GeV}$ - seems appealing.

There are 19 parameters in the SM Lagrangian, such as masses and mixing angles. The values of these parameters have to be determined from measurements, as there is no way to predict them in the SM.

The SM can also not explain why for instance the charge of a proton - which is a bound stand of three quarks - cancels exactly the charge of an electron, although in the SM there is no direct link between quarks and leptons, except for anomaly freedom. 
While these properties of the SM can be regarded as theoretically aesthetical problems which by themselves do not falsify the SM as a complete theory of nature, there is a number of observations that can not be explained by the SM. One of these observations is the apparent absence of anti-matter in the universe. The common models dealing with the origins of the universe and the creation of baryonic matter predict an equal number of particles and anti-particles, which contradicts the widespread interpretation of currently available observations. While it cannot be completely ruled out by these observations that there are anti-matter dominated regions in the universe, it would not be unexpected to spot the borders between matter dominated and anti-matter dominated regions in the spectrum of the cosmic microwave background (CMB). Since the CMB does not show any hint on such a region, it is widely believed that the universe is mainly made up of matter rather than anti-matter. Although the SM does provide a CP violating mechanism via complex entries in the CKM Matrix (2.22), the amount of CP violation as predicted by the SM according to measurements in $B$ and $K$ physics, does not suffice to explain the absence of anti-matter in the universe (for a review, see for instance [34]).

Furthermore, according to the measurements of the CMB, performed with the COBE and WMAP satellites, the visible baryonic matter does only make up $5 \%$ of the energy in the universe. Roughly $20 \%$ are today believed to be made of dark matter while the remaining $75 \%$ are referred to as dark energy [37, 38, 39]. The assumption of the existence of dark matter is supported by different observations, which are the rotation curves of galaxies as well as gravitational lensing effects in areas of the universe that lack a sufficient amount of visible matter to explain the observations. As of today dark matter has not been directly observed under laboratory conditions, and its nature remains unknown so far. However none of the particles known in the SM can be accounted for the full amount of dark matter observed. It is therefore widely believed that additional particles must exist, the properties of which must be significantly different compared to the properties of the particles that are known in the SM.

\subsection{Extensions of the Standard Model}

There are several interesting ideas for the extension of the SM towards a more complete theory of nature. It is widely suspected that at some point the strong interaction can be unified with the electroweak interaction in a similar manner as the weak interaction was unified with the electromagnetic interaction in the Glashow-Weinberg-Salam Model. In such a 'Grand Unified Theory' (GUT) the coupling constants of all interactions would unify at an energy scale $Q_{\mathrm{GUT}}$. This does not seem to happen in the SM, as shown in Figure 2.1, but if there exist extensions to the SM which modify the $\beta$-functions for the coupling constants, such a unification can typically be achieved at $Q_{\mathrm{GUT}} \sim 10^{16} \mathrm{GeV}$ or above. There are several models for a GUT, the first version of which features a superior $S U(5)$ symmetry that breaks down to the known $S U(3)_{C} \times$ $S U(2)_{L} \times U(1)_{Y}$ symmetry at lower scales, but that is only one possibility. Depending on the assumed superior symmetry group, quarks and leptons are put into one or several multiplets, which provides a natural explanation for the cancellation of the charges of the proton and the electron, for instance. The fundamental problems with GUTs are usually the prediction of both the proton decay and the existence of a large number of residual magnetic monopoles in the universe, for both of which there is no definite experimental proof as of today.

Even a step further, including a quantised version of gravity, string theories are setup as the paradigm for a 'Theory of Everything'. The concept of point-like particles is replaced by ndimensional branes, usually requiring the extension of space-time by at least 5 dimensions. The main flaw of string theories, however, is the lack of testable predictions at low energy scales. 


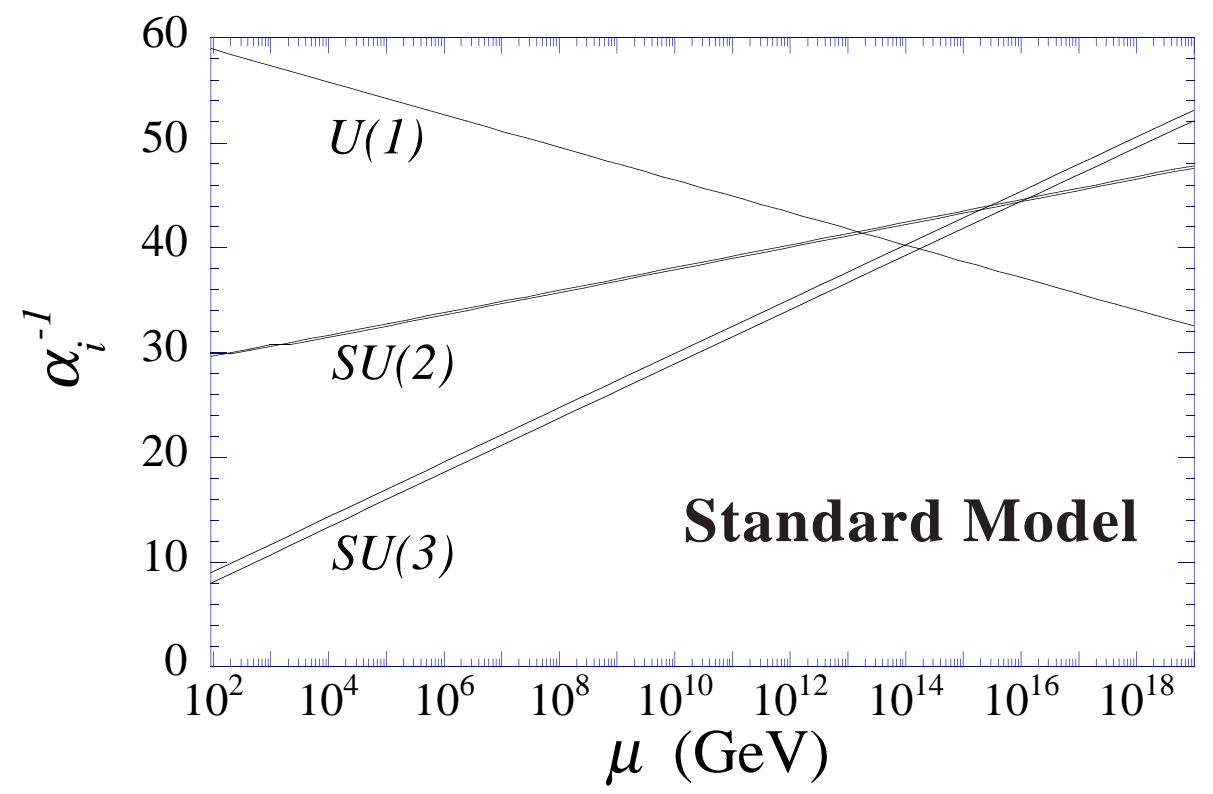

Figure 2.1: The running of the gauge couplings in the Standard-Modell. If the SM was valid up to energies at which quantum gravity is believed to provide significant contributions $\left(10^{16}\right.$ $\mathrm{GeV}$ ), there would be no unification of the gauge couplings. Although no strict reason can be given, it seems somehow uncomfortable that a unification does not appear at some high scale. The plot is taken from [40].

Technicolor Apart from extensions that manifest at energies near the Planck scale, low energy extensions with experimental signatures that are accessible at contemporary experiments have been constructed. One group of these extensions, referred to as Technicolor (TC) (see for instance [34]), breaks electroweak symmetry dynamically. In these models, the scalar added to the massless theory is not an elementary state, but it is a bound state of new fundamental fermions, the techniquarks. A new gauge interaction is introduced on the basis of QCD; the new interaction is asymptotically free at high energies and gets stronger (confining) at low energies. The chiral symmetry of the fermions is spontaneously broken, which according to Goldstone's theorem gives rise to massless bosons, which under certain conditions get absorbed in the longitudinal polarisation of the $W$ and $Z$ bosons. Additional interactions are needed to give mass to the SM fermions, which is usually referred to as extended TC. Besides avoiding the hierarchy problem, TC models can include candidates for dark matter [41]. Usually the dark matter candidate in TC models is a technicolourless technibaryon. Depending on the scale of $\mathrm{TC}$, this candidate can achieve masses in the $\mathrm{TeV}$ regime and could therefore be produced at the LHC. In order to match the experimental results, TC models have become increasingly complex. An overview on the various aspects of TC models is given in [34].

Extra Dimensions Similar to string theories some extensions of the SM introduce extra dimensions that are usually curled up. In the simplest of these models, the extra dimensions are characterised by a compactification radius $R$ that is closely related to the mass scale of additional quantum states, if the SM fields are assumed to propagate in the extra dimensions. A series of so-called Kaluza-Klein excitations, of which only those with lower masses are phenomenologically of interest, are predicted. Assuming momentum conservation in the extra dimensions, KaluzaKlein excitations that propagate in these extra dimensions can only be produced in pairs at 
least, which is sometimes referred to as conservation of Kaluza-Klein parity. This implies that the lightest Kaluza-Klein state is stable and therefore a possible candidate for dark matter. Such models are usually referred to as models of Universal Extra Dimensions (UED) and their phenomenology is closely related to that of supersymmetry, which is described in the next section. For an overview on extra dimensions, see for instance [34, 42, 43].

It is also possible to constrain the SM fields to the four known space-time dimensions. In that case, the models are usually called models of Large Extra Dimensions, or ADD models [44].

Supersymmetry Another possible extension of the SM features the introduction of a new symmetry between fermions and bosons called Supersymmetry (SUSY). This is explained in detail in the next chapter.

\subsection{The Minimal Supersymmetric Standard Model}

The essential feature of SUSY is that it introduces a symmetry between the two fundamental types of particles known in the SM, fermions and bosons. Each bosonic (fermionic) degree of freedom is complemented with a fermionic (bosonic) degree of freedom that apart from the spin has the same quantum numbers. The action integral (2.1) is required to remain unchanged if a boson (fermion) is transformed into the corresponding fermion (boson), which is called its superpartner. Based on a simple supersymmetric model by Wess and Zumino [45], the Minimal Supersymmetric Standard Model is built by imposing invariance under SUSY transformations on the SM Lagrangian.

The hierarchy problem of the SM is solved in a similar manner as gauge symmetry prohibits unnatural mass terms for the gauge bosons and chiral symmetry forbids unnatural corrections to the fermion masses. Although it is already known not to be an exact symmetry, SUSY is still considered to be one of the most interesting extensions of the SM, as in addition to unifying the gauge couplings at a large energy scale, it can provide a good candidate for dark matter. Furthermore SUSY has a rich phenomenology at hadron as well as lepton colliders.

In this section, after introducing the SUSY algebra and the superfield formalism, the Minimal Supersymmetric Standard Model, SUSY breaking and SUSY phenomenology at hadron colliders is described. The aim of this chapter is to provide a rough overview of supersymmetry with a focus on several features that are of importance in the remainder of this thesis. This description relies mainly on $[8,15,18]$, where additional information - in particular on the simplified SUSY breaking scenarios - can be found.

\subsubsection{Supersymmetry}

It was shown by Coleman and Mandula that in the case of bosonic generators any continuous symmetry of the scattering matrix $S$ must be a direct product of the Poincare group and an internal symmetry [46]. That is, the charges associated to such symmetry transformations are Lorentz scalars under elements of the Poincaré group. If, on the other hand, the generators transform like spinors, it was shown by Haag, Łopuszański and Sohnius that there exists a non-trivial extension of the Poincaré algebra, which is the SUSY algebra [47].

The key feature of the SUSY algebra is that its generators transform as spin- $\frac{1}{2}$ objects, such that in contrast to bosonic generators of symmetry groups, which obey commutation relations, the SUSY algebra is defined by anti-commutation relations. For SUSY generators $Q^{A}$ and $Q^{A \dagger}$, where $A$ is an index running over the number $N$ of independent SUSY transformations, such 
that

$$
Q^{A} \mid \text { boson }>=\mid \text { fermion }>\quad Q^{A} \mid \text { fermion }>=\mid \text { boson }>
$$

the anti-commutation relations

$$
\begin{aligned}
\left\{Q_{\alpha}^{A}, Q_{\dot{\alpha}}^{B \dagger}\right\} & =-2 \sigma \delta_{A}^{B}\left(\sigma^{\mu}\right)_{\alpha \dot{\alpha}} P^{\mu} \\
\left\{Q_{\alpha}^{A}, Q_{\beta}^{B}\right\} & =-2 \varepsilon_{a b} Z^{A B} \\
\left\{Q_{\dot{\alpha}}^{A \dagger}, Q_{\dot{\beta}}^{B \dagger}\right\} & =-2 \varepsilon_{\dot{\alpha} \dot{\beta}}\left(Z^{A B}\right)^{*}
\end{aligned}
$$

with

$$
\varepsilon_{11}=\varepsilon_{22}=0, \quad \varepsilon_{12}=-\varepsilon_{21}=-1 \quad \text { and } Z^{A B}=-Z^{B A}
$$

must hold. Due to the $V-A$ structure of the $S U(2)_{L}$ interactions, it is in general difficult to create models with $N>1$, such that in the following only one SUSY transformation will be considered and the index $A$ will be omitted. Furthermore, since SUSY is a global symmetry, the generators commute with the generators of space-time translations,

$$
\left[Q_{\alpha}, P^{\mu}\right]=0, \quad\left[Q_{\dot{\alpha}}^{\dagger}, P^{\mu}\right]=0 .
$$

Usually, bosons and fermions that are related by equations (2.28) are combined in a supermultiplet and are referred to as superpartners. Equations (2.33) imply that particles in the same supermultiplet must have the same mass. Furthermore, the operators $Q_{\alpha}$ and $Q_{\dot{\alpha}}^{\dagger}$ commute with the generators of the known gauge transformations, which means that superpartners also have the same gauge quantum numbers. There are three different types of supermultiplets that are needed for the minimal supersymmetric extension of the SM:

First, a chiral supermultiplet consists of a complex scalar field and a two-component Weyl fermion field. Second, a vector supermultiplet contains a vector field and a two-component Weyl fermion field. Third, if the hypothetical graviton is to be included in the theory, a supermultiplet made of a tensor boson and its spin- $\frac{3}{2}$ partner is needed. As they are of major importance for the remainder of this thesis, the structure of chiral and vector multiplets is described in a bit more detail in the following, while details on the graviton/gravitino supermultiplet are of limited importance for this thesis.

It can be shown that the number of bosonic degrees of freedom in a supermultiplet must equal the number of fermionic degrees of freedom in the same supermultiplet due to equations $(2.33)$ and (2.30). For a supersymmetrised theory to be valid on-shell as well as off-shell, this forces the introduction of 'auxiliary' fields $F$ and $D$. These fields do not propagate, but they transform in a non-trivial way under SUSY transformations.

The formulation of a supersymmetric theory is widely done using the notation of superfields. In analogy to space-time transformations $x^{\mu} \rightarrow x^{\mu}+a^{\mu}$, which are generated by the four-momentum $P^{\mu}$, the SUSY operators $Q_{\alpha}$ and $Q_{\dot{\alpha}}^{\dagger}$ are considered to generate translations in superspace, which is defined by the space-time coordinates $x^{\mu}$ extended by two additional, anti-commuting twocomponent fermionic dimensions, $\theta^{\alpha}$ and $\theta_{\dot{\alpha}}^{\dagger}$. All fields in a supermultiplet are then combined in a superfield $S=S\left(x, \theta, \theta^{\dagger}\right)$, such that an infinitesimal translation in superspace is a global SUSY transformation. Due to the anti-commuting nature of the fermionic coordinates, the most general form for a superfield is given by

$$
\begin{aligned}
S\left(x, \theta, \theta^{\dagger}\right)= & a(x)+\theta \xi(x)+\theta^{\dagger} \chi^{\dagger}(x)+\theta \theta b(x)+\theta^{\dagger} \theta^{\dagger} c(x)+\theta^{\dagger} \bar{\sigma}^{\mu} \theta v_{\mu}(x) \\
& +\theta^{\dagger} \theta^{\dagger} \theta \eta(x)+\theta \theta \theta^{\dagger} \zeta^{\dagger}(x)+\theta \theta \theta^{\dagger} \theta^{\dagger} d(x),
\end{aligned}
$$


where $a, b, c, d$ and $v_{\mu}$ are bosonic fields with a total of 16 degrees of freedom, and $\xi, \chi^{\dagger}, \eta$ and $\zeta^{\dagger}$ are two-component fermion fields, also with 16 degrees of freedom. It can be shown by imposing the appropriate constraints on (2.34), that the most general form of a left chiral superfield, describing a left chiral supermultiplet, is given by

$$
\Phi(x, \theta)=\phi(x)+\sqrt{2} \theta \chi(x)+\theta \theta F(x),
$$

in which $\phi$ is a scalar, $\chi$ is a two-component Weyl spinor and $F$ is the above mentioned auxiliary field. $F$ is sometimes also referred to as the $F$-component of a chiral superfield, and the term containing the $F$ is referred to as the $F$-term of the superfield. Similarly, a real vector - or gauge - supermultiplet is most generally described by

$$
V\left(x, \theta, \theta^{\dagger}\right)=\theta^{\dagger} \bar{\sigma}^{\mu} \theta A_{\mu}+\theta^{\dagger} \theta^{\dagger} \theta \lambda^{\dagger}+\frac{1}{2} \theta \theta \theta^{\dagger} \theta^{\dagger} D
$$

with the vector field $A_{\mu}$, the two-component Weyl fermion $\lambda$ and the auxiliary field $D$.

If local gauge invariance is imposed on a supersymmetric Lagrangian, the gauge interactions arise as described above by replacing the derivative with the covariant derivative $D_{\mu}$. This introduces interactions terms between both the fermions as well as the bosons in a chiral supermultiplet with the vector fields from the respective gauge supermultiplet. In addition to these gauge interactions, terms introducing interactions between the components of the chiral superfield and the other components of the gauge superfield are also allowed.

After imposing local gauge invariance, for an infinitesimal SUSY transformation described by the fermionic parameter $\varepsilon$, performed on a system with both chiral and gauge superfields, the changes in the field variables are

$$
\begin{aligned}
\delta \phi_{i} & =\varepsilon \chi_{i}, \\
\delta\left(\chi_{i}\right)_{\alpha} & =-i\left(\sigma^{\mu} \varepsilon^{\dagger}\right)_{\alpha} D_{\mu} \phi_{i}+\varepsilon_{\alpha} F_{i}, \\
\delta F_{i} & =-i \varepsilon^{\dagger} \bar{\sigma}^{\mu} D_{\mu} \chi_{i}+\sqrt{2} g\left(T^{a} \phi\right)_{i} \varepsilon^{\dagger} \lambda^{\dagger a}, \\
\delta A_{\mu}^{a} & =-\frac{1}{\sqrt{2}}\left(\varepsilon^{\dagger} \bar{\sigma}_{\mu} \lambda^{a}+\lambda^{\dagger a} \bar{\sigma}_{\mu} \varepsilon\right), \\
\delta \lambda_{\alpha}^{a} & =\frac{i}{2 \sqrt{2}}\left(\sigma^{\mu} \bar{\sigma}^{\nu} \varepsilon\right)_{\alpha} F_{\mu \nu}^{a}+\frac{1}{\sqrt{2}} \varepsilon_{\alpha} D^{\alpha}, \\
\delta D^{a} & =\frac{i}{\sqrt{2}}\left(-\varepsilon^{\dagger} \bar{\sigma}^{\mu} D_{\mu} \lambda^{a}+D_{\mu} \lambda^{\dagger a} \bar{\sigma}^{\mu} \varepsilon\right) .
\end{aligned}
$$

Apart from the gauge interactions, it is possible to add more gauge and SUSY invariant interaction terms by constructing a so-called superpotential from products of chiral superfields. The most general form of such a superpotential for a system of chiral superfields $\Phi_{i}$ is given by a linear term and the product of two and/or three chiral superfields, of which due to SUSY only the $F$-terms may appear in the Lagrangian density,

$$
\begin{aligned}
W & =L_{i} \Phi_{i}+\frac{1}{2} M_{i j} \Phi_{i} \Phi j+\frac{1}{6} y_{i j k} \Phi_{i} \Phi_{j} \Phi_{k}, \\
\mathcal{L}_{\text {int }} & =\left.W\right|_{F},
\end{aligned}
$$

where $\left.W\right|_{F}$ is the sum of all $F$-terms in the superpotential. It is important to notice that SUSY requires the superpotential to be a holomorphic function of the scalar parts of the superfields, which - using equation (2.35) - means that it may only depend on $\phi$ and not on $\phi^{*}$. 
For completeness, the full Lagrangian for a supersymmetric theory is

$$
\begin{aligned}
\mathcal{L}_{\mathrm{SUSY}} & =-D^{\mu} \phi_{i}^{*} D_{\mu} \phi_{i}+i \psi^{\dagger i} \bar{\sigma}^{\mu} D_{\mu} \psi_{i}-\frac{1}{2}\left(W^{i j} \psi_{i} \psi_{j}+W_{i j}^{*} \psi^{\dagger i} \psi^{\dagger j}\right)-W^{i} W_{i}^{*} \\
& -\frac{1}{4} F_{\mu \nu}^{a} F^{\mu \nu a}+i \lambda^{\dagger a} \bar{\sigma}^{\mu} D_{\mu} \lambda^{a}+\frac{1}{2} D^{a} D^{a} \\
& -\sqrt{2} g_{a}\left(\phi^{*} T^{a} \psi\right) \lambda^{a}-\sqrt{2} g_{a} \lambda^{\dagger a}\left(\psi^{\dagger} T^{a} \phi\right)+g_{a}\left(\phi^{\dagger} T^{a} \phi\right) D^{a},
\end{aligned}
$$

where a summation over the gauge group index $a$, and the family index $i$ is implied, and the definitions

$$
\begin{aligned}
W^{i} & =\frac{\left.\partial W\right|_{F}}{\partial \phi_{i}} \\
W^{i j} & =\frac{\left.\partial^{2} W\right|_{F}}{\partial \phi_{i} \phi_{j}}
\end{aligned}
$$

have been used. Using the equation of motion for the auxiliary field

$$
D^{a}=-g\left(\phi_{i}^{*} T^{a} \phi_{i}\right)
$$

all terms that are pure polynomials of the scalar fields $\phi_{i}$ and $\phi_{i}^{\dagger}$ can be summarised in a scalar potential,

$$
V\left(\phi_{i}, \phi_{i}^{\dagger}\right)=\underbrace{W_{i}^{*} W^{i}}_{\text {F-terms }}+\underbrace{\frac{1}{2} g_{a}^{2}\left(\phi^{*} T^{a} \phi\right)^{2}}_{\text {D-terms }}
$$

The F-terms are usually fixed by the fermion mass terms and the Yukawa couplings in the theory, while the gauge interactions fix the D-terms.

In addition to the superpotential, for some considerations it is neccessary to add non-renormalisable terms to the Lagrangian, making it an effective theory. This is in general possible by adding a Kähler potential and a gauge kinetic function to the Lagrangian. More details on this can be found in $[8]$.

\subsubsection{The Minimal Supersymmetric Standard Model}

The Minimal Supersymmetric Standard Model (MSSM) is constructed by requiring the SM Lagrangian (2.21) to be supersymmetric. It is minimal in the sense that the particle content of the SM is extended in the smallest possible way. With the superfields as defined in Table 2.4, the superpotential of the MSSM is given by

$$
W_{\mathrm{MSSM}}=\bar{u} \mathbf{y}_{\mathbf{u}} Q H_{u}-\bar{d} \mathbf{y}_{\mathbf{d}} Q H_{d}-\bar{e} \mathbf{y}_{\mathbf{e}} L H_{d}+\mu H_{u} H_{d},
$$

where a sum over three families quarks/squarks and leptons/sleptons is implied. Two different Higgs doublets $H_{u}$ and $H_{d}$ are needed, as due to the requirement of the superpotential being holomorphic, terms like the second one in equation (2.20) are forbidden. Also, by inserting equation (2.47) into equation (2.46), it can be shown that this superpotential cannot provide electroweak symmetry breaking - the vacuum expectation value (vev) for both Higgs fields is zero. This is resolved when SUSY breaking is considered, c.f. chapter 2.3.3.

The fermions in the SM are combined with complex scalar fields. Since they couple differently to the electroweak gauge bosons, each chiral state of the fermion is assigned its own superpartner. 


\begin{tabular}{|c|c|c|c|c|c|}
\hline Superfield & & spin 0 & $\operatorname{spin} \frac{1}{2}$ & spin 1 & $S U(3)_{C}, S U(2)_{L}, U(1)_{Y}$ \\
\hline squarks/quarks & $Q$ & $\left(\begin{array}{ll}\tilde{u}_{L} & \tilde{d}_{L}\end{array}\right)$ & $\left(\begin{array}{ll}u_{L} & d_{L}\end{array}\right)$ & & $\left(3,2, \frac{1}{6}\right)$ \\
\hline 3 families & $\bar{u}$ & $\tilde{u}_{R}^{*}$ & $u_{R}^{\dagger}$ & & $\left(\mathbf{3}, \mathbf{1},-\frac{2}{3}\right)$ \\
\hline & $\bar{d}$ & $\tilde{d}_{R}^{*}$ & $d_{R}^{\dagger}$ & & $\left(\mathbf{3}, \mathbf{1}, \frac{1}{3}\right)$ \\
\hline sleptons/leptons & $L$ & $\left(\begin{array}{ll}\tilde{\nu} & \tilde{e}_{L}\end{array}\right)$ & $\left(\begin{array}{ll}\nu & e_{L}\end{array}\right)$ & & $\left(\mathbf{1}, \mathbf{2},-\frac{1}{2}\right)$ \\
\hline 3 families & $\bar{e}$ & $\tilde{e}_{R}^{*}$ & $e_{R}^{\dagger}$ & & $(\mathbf{1}, \mathbf{1}, 1)$ \\
\hline \multirow[t]{2}{*}{ Higgs, Higgsinos } & & $\left(\begin{array}{ll}H_{u}^{+} & H_{u}^{0}\end{array}\right)$ & $\left(\begin{array}{ll}\tilde{H}_{u}^{+} & \tilde{H}_{u}^{0}\end{array}\right)$ & & $\left(1,2, \frac{1}{2}\right)$ \\
\hline & & $\left(\begin{array}{ll}H_{d}^{0} & H_{d}^{-}\end{array}\right)$ & $\left(\tilde{H}_{d}^{0} \tilde{H}_{d}^{-}\right.$ & & $\left(1,2,-\frac{1}{2}\right)$ \\
\hline "gluinos/gluons & & & $\tilde{g}$ & $g$ & $\overline{\overline{(8,1,0)}}$ \\
\hline Winos/W bosons & & & $\tilde{W}^{ \pm} \tilde{W}^{0}$ & $W^{ \pm} W^{0}$ & $(1,3,0)$ \\
\hline Bino/B boson & & & $\tilde{B}^{0}$ & $B$ & $(1,1,0)$ \\
\hline
\end{tabular}

Table 2.4: The superfield content in the MSSM with the gauge quantum numbers

The superpartners of left (right) chiral fermions get an index $L(R)$ and are referred to as left (right) chiral sfermions, although they cannot be subject to the chirality operator (2.13). The complex scalar Higgs fields are combined with fermionic fields called Higgsinos. Finally, the gauge bosons are matched with fermion fields that are called gauginos. The graviton and its superpartner, the gravitino, are omitted in the table.

Despite the lack of a non-zero Higgs vev, the supersymmetrisation of the SM into the MSSM has some remarkable consequences, which will briefly be mentioned here: In the MSSM the masses of the fundamental scalars in the theory are no longer quadratically divergent in terms of the cut-off scale $\Lambda_{C}$, up to which the theory is considered to be valid. For a fundamental scalar that couples to a fermion via some Yukawa coupling as introduced in the SM by equations (2.20), the mass of that scalar receives corrections from one-loop diagrams that are of the form

$$
\Delta_{\text {fermion }}=-\frac{y^{2}}{8 \pi^{2}} \lambda_{C}^{2},
$$

which in an unbroken supersymmetry get exactly canceled by loop corrections involving the scalar superpartner of the fermion

$$
\Delta_{\text {boson }}=\frac{y^{2}}{8 \pi^{2}} \lambda_{C}^{2} .
$$

The hierarchy problem of the SM is solved by SUSY, although the original motivation for exploring supersymmetric models in the first place was not the solution of the SM hierarchy problem. Furthermore, the beta-functions (2.23) for the three known gauge couplings $\alpha_{1}, \alpha_{2}$ and $\alpha_{3}$ are modified, as all of them contain sums over the number of fermion gauge multiplets as well as scalar gauge multiplets. The latter are usually introduced by the MSSM only, such that at $Q_{G U T}^{M S S M} \sim 10^{16} \mathrm{GeV}$ all three coupling constants are roughly unified, as shown in Figure 2.2 .

In principle, both lepton number as well as baryon number violating terms are allowed as an addition to the MSSM superpotential (2.47). These terms usually are

$$
\Delta W=\lambda_{B}^{i j k} \bar{u}_{i} \bar{d}_{j} \bar{d}_{k}
$$

and similar ones. Since their inclusion would predict proton decay, such terms are highly constrained by current observations. It is possible to eliminate such terms in the Lagrangian by 


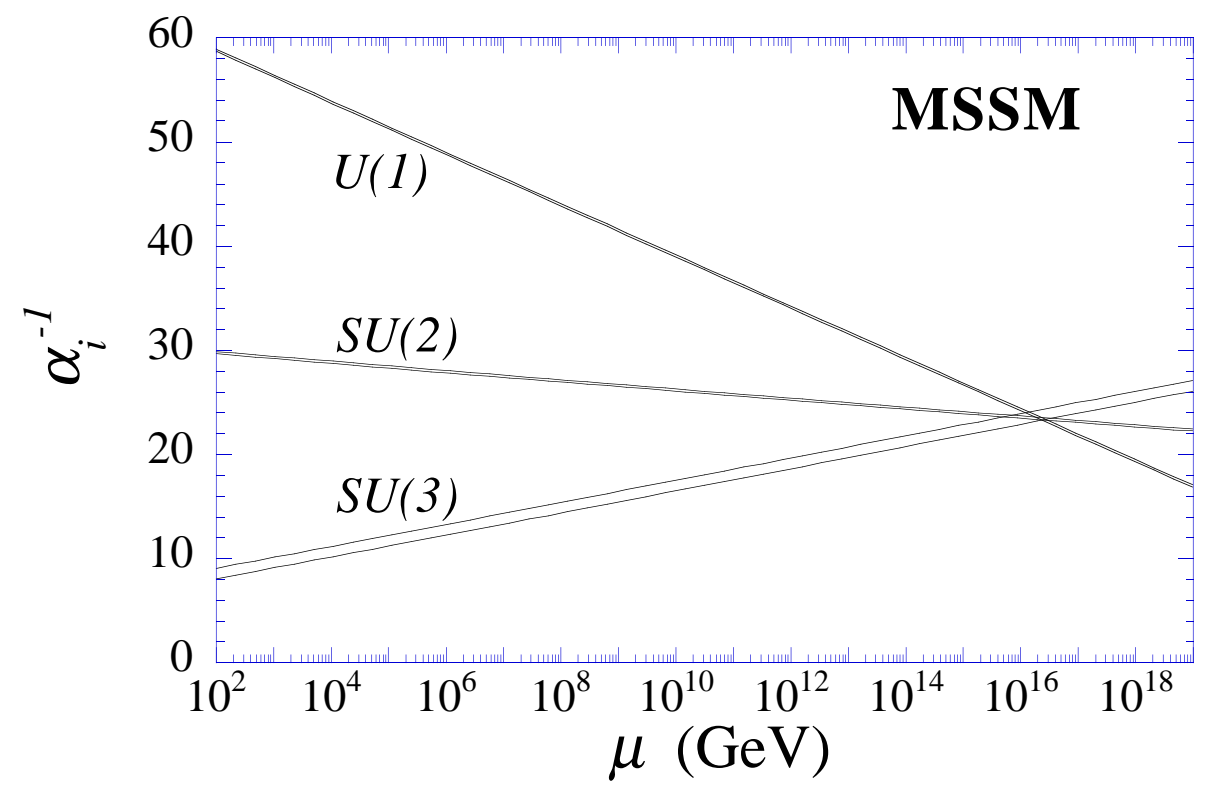

Figure 2.2: The diagram shows the running of the gauge-couplings in the MSSM. Within their theoretical uncertainties, the three coupling constants unify at energy scales $\gtrsim 10^{16} \mathrm{GeV}$. The exact value depends on the actual breaking mechanism and the parameters of the SUSY Lagrangian density. The unification of the gauge couplings at a high scale is one of the convincing arguments to consider SUSY seriously. However, there is no evidence that such a unification has to appear in nature, but it seems to be more natural to a certain extend. The plot is taken from [40].

introducing another conserved quantum number called $R$-parity. It is defined as

$$
R=(-1)^{3 B+L+2 s},
$$

where $B$ is the baryon number, $L$ is the lepton number and $s$ is the spin. The known particles in the SM would have $R=1$, while their superpartners would get $R=-1$. If $R$-parity was conserved, the lightest superpartner would be stable, and under certain conditions a good candidate for dark matter.

\subsubsection{Supersymmetry Breaking}

The particles in the SM cannot be combined to supermultiplets as can be seen from their attribution to the gauge group representations in Table 2.4. Since no scalars with the same gauge quantum numbers and the same masses as the SM fermions have been found, and since also no fermions with the same gauge quantum numbers and masses as the SM bosons have been found, SUSY cannot be an exact symmetry but it must be broken.

One way to break a symmetry is to do it spontaneously. For SUSY, spontaneous symmetry breaking can be achieved in two different ways, i.e. either of the auxiliary fields $F$ or $D$ can achieve a vev - in the first (latter) case referred to as $F$-type ( $D$-type) SUSY breaking. Details on how either of these can be achieved can be found in [8] and [18]. There is a number of different proposals for the exact mechanism of SUSY breaking, which is generally assumed to happen in a hidden sector and be mediated by some flavour blind interactions to the visible sector as shown in Figure 2.3. In order to explore the phenomenology of the MSSM, the theory 
is made an effective theory by introducing terms in the Lagrangian that explicitly break the symmetry without spoiling local gauge invariance or renormalisability, which is the second way to break a symmetry.

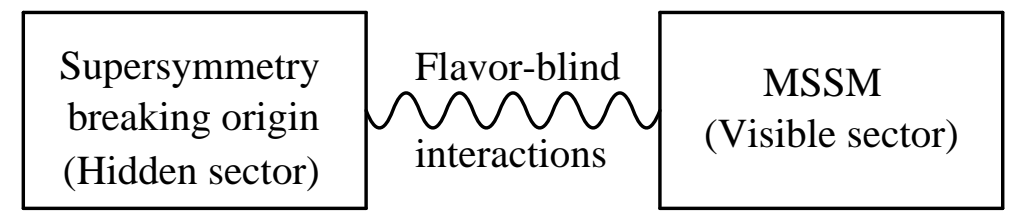

Figure 2.3: An illustration of the SUSY breaking mechanism. The actual breaking of the symmetry is assumed to take place in some hidden sector (left hand side), which is not directly accessible. Via flavour blind interactions, the SUSY breaking is mediated to the visible sector on the right hand side.

Usually only soft SUSY breaking terms are allowed, such that

$$
\mathcal{L}_{\text {effective }}=\mathcal{L}_{\text {SUSY }}+\mathcal{L}_{\text {soft }},
$$

where the term soft indicates that such terms do not reintroduce quadratic divergences in any of the SUSY loop corrections. For instance, with a SUSY mass scale $m_{\text {soft }}$, the soft SUSY breaking terms introduces contributions to the mass of a fundamental scalar that are proportional to $m_{\text {soft }}^{2} \ln \frac{\Lambda_{C}}{m_{\text {soft }}}$, only. Thus, if $m_{\text {soft }}$ is not too large, broken SUSY can still be seen as a solution to the hierarchy problem in the SM. The most general form of the explicit SUSY breaking terms is given by [18]

$$
\begin{aligned}
\mathcal{L}_{\text {soft }} & =-\frac{1}{2}\left(M_{3} \tilde{g}^{a} \cdot \tilde{g}^{a}+M_{2} \tilde{W}^{a} \cdot \tilde{W}^{a}+M_{1} \tilde{B} \cdot \tilde{B}+\text { h.c }\right) \\
& -m_{\tilde{Q}_{i j}}^{2} \tilde{Q}_{i}^{\dagger} \cdot \tilde{Q}_{j}-m_{\tilde{u}_{i j}}^{2} \tilde{u}_{R i} \tilde{u}_{R j}^{\dagger}-m_{\tilde{d}_{i j}}^{2} \tilde{d}_{R i} \tilde{d}_{R j}^{\dagger} \\
& -m_{\tilde{L}_{i j}}^{2} \tilde{L}_{i}^{\dagger} \tilde{L}_{j}-m_{\tilde{e}_{i j}}^{2} \tilde{e}_{R i} \tilde{e}_{R f}^{\dagger} \\
& -m_{H_{u}}^{2} H_{u}^{\dagger} \cdot H_{u}-m_{H_{d}}^{2} H_{d}^{\dagger} \cdot H_{d}-\left(b H_{u} \cdot H_{d}+\text { h.c. }\right) \\
& -a_{u}^{i j} \tilde{u}_{R i}^{\dagger} \tilde{Q}_{j} \cdot H_{u}+a_{d}^{i j} \tilde{d}_{R i}^{\dagger} \tilde{Q}_{j} \cdot H_{d}+a_{e}^{i j} \tilde{e}_{R i}^{\dagger} \tilde{L}_{j} \cdot H_{d}+\text { h.c.. }
\end{aligned}
$$

While these terms explicitly break SUSY, they preserve local gauge invariance if the squark and slepton mass matrices are hermitian. This is, in contrast to terms like (2.17), because the sfermions and bosinos are parts of the adjoint representation of the gauge groups. Some of the terms in (2.53) have exactly the same structure as other terms in the MSSM superpotential (2.47), but they only include the superpartners of the known SM particles and therefore break SUSY. For the Higgs superfields explicit mass terms for the scalars are allowed, whereas such terms for the Higgsinos are forbidden by gauge invariance. It is by these extra terms that the local $S U(2)_{L}$ gauge invariance is spontaneously broken in the MSSM and the Higgs fields acquire non-zero vacuum expectation values $v_{u}$ and $v_{d}$. While the unbroken MSSM cannot provide electroweak symmetry breaking, this can be achieved by breaking SUSY as well. This is sometimes interpreted as a hint that, if SUSY turns out to be approximately realised in nature, the mechanisms for electroweak symmetry breaking and SUSY breaking are closely related to each other. As the masses of the electroweak gauge bosons are known, so is the combination of the two vevs,

$$
\left(v_{u}^{2}+v_{d}^{2}\right)^{\frac{1}{2}}=\left(\frac{2 m_{W}^{2}}{g^{2}}\right)^{\frac{1}{2}}=174 \mathrm{GeV} .
$$


The ratio of the vacuum expectation values is usually referred to as

$$
\tan \beta=\frac{v_{u}}{v_{d}} \quad \text { with } \beta \in\left(0, \frac{\pi}{2}\right) .
$$

The soft SUSY breaking terms add a total of 105 new parameters to the unbroken SUSY Lagrangian, which only has one additional parameter compared to the SM. These parameters are the gaugino mass parameters $M_{1}, M_{2}$ and $M_{3}$, the entries of the sfermion mass matrices $\mathbf{m}_{\tilde{Q}}^{2}, \mathbf{m}_{\tilde{u}}^{2}, \mathbf{m}_{\tilde{d}}^{2}, \mathbf{m}_{\tilde{L}}^{2}$ and $\mathbf{m}_{\tilde{e}}^{2}$, the Higgs mass parameters $m_{H_{u}}, m_{H_{d}}$ and $b$, and the entries of the trilinear coupling matrices $\mathbf{a}_{u}, \mathbf{a}_{d}$ and $\mathbf{a}_{e}$. For phenomenological considerations, it is common to replace the parameters $m_{H_{u}}, m_{H_{d}}$ and $b$ with the parameters $\mu, \tan \beta$ and $m_{A^{0}}$ using the equations

$$
\begin{aligned}
|\mu|^{2}+m_{H_{u}}^{2} & =b \cot \beta+\frac{m_{Z}^{2}}{2} \cos 2 \beta \\
|\mu|^{2}+m_{H_{d}}^{2} & =b \cot \beta-\frac{m_{Z}^{2}}{2} \cos 2 \beta \\
m_{A^{0}}^{2} & =\frac{2 b}{\sin 2 \beta} .
\end{aligned}
$$

There are strong constraints on nearly all of the parameters introduced in (2.53). For instance, the sfermion mass matrices can in principle be complex matrices. Usually such matrices can add large $\mathrm{CP}$ violating contributions, as well as large contributions to flavour changing neutral currents (FCNC). In addition, off-diagonal terms in the mass matrices give rise to both lepton flavour as well as quark flavour changing processes involving the superpartners, which by loop corrections contribute to such processes involving the known SM particles. It is by data from a growing number of experiments that such terms are highly constrained.

It is sometimes assumed that these constraints are hints to a SUSY breaking mechanism that avoids large $\mathrm{CP}$ and flavour violating terms in a natural way. A selection of such models is detailed in the remainder of this chapter, after describing some of the phenomenology of the MSSM. It is important to note that as parameters of the Lagrangian, all soft SUSY breaking parameters receive loop corrections and due to renormalisation, are running functions of the energy scale $Q$.

\section{The pMSSM}

For phenomenological considerations, it is sometimes useful to set all the complex phases in the MSSM to 0. Also, the mixing angles and the trilinear terms and Yukawa couplings for the first two generations are sometimes set to 0 . The smallness of these terms, and the strong constraints that already exist on them justify such a simplification, without loosing too much universality. The MSSM as simplified in this way is then referred to as the phenomenological MSSM, or pMSSM.

Depending on additional assumptions about the unification of certain parameters, there are 24 or less free parameters left in the pMSSM. These are

- 9 squark mass parameters $M_{Q, i i}, M_{U, i i}$ and $M_{D, i i}$ with $i \in[1,3]$

- 6 slepton mass parameters $M_{L, i i}$ and $M_{E, i i}$ with $i \in[1,3]$

- 3 gaugino mass parameters, $M_{1}, M_{2}$ and $M_{3}$

- 3 trilinear couplings, $A_{t}, A_{b}$ and $A_{\tau}$ 
- 3 parameters in the Higgs sector, $m_{A^{0}}, \mu$ and $\tan \beta$.

If the mass parameters of the first and the second generation are assumed to be equal, as it is sometimes done, the model has only 19 parameters left.

\subsubsection{The Phenomenology of the MSSM}

Using the SUSY Lagrangian (2.45) and the soft SUSY breaking terms (2.53) the phenomenology of the MSSM is described in this section. After a short description of the MSSM mass spectrum which includes mixing matrices in nearly all sectors, a short overview of the production and the decay of supersymmetric particles at a proton-proton collider is given.

\section{The Mass spectrum of the MSSM}

The Higgs Sector In the MSSM there is a total of eight real scalar degrees of freedom in the two Higgs doublets $\left(H_{u}^{+} H_{u}^{0}\right)$ and $\left(H_{d}^{0} H_{d}^{-}\right)$. The mass eigenstates of the MSSM Higgs bosons are mixtures of the gauge eigenstates, such that following the notation of [8]

$$
\begin{aligned}
\left(\begin{array}{c}
H_{u}^{0} \\
H_{d}^{0}
\end{array}\right) & =\left(\begin{array}{l}
v_{u} \\
v_{d}
\end{array}\right)+\frac{1}{\sqrt{2}} R_{\alpha}\left(\begin{array}{c}
h^{0} \\
H^{0}
\end{array}\right)+\frac{i}{\sqrt{2}} R_{\beta 0}\left(\begin{array}{c}
G^{0} \\
A^{0}
\end{array}\right), \\
\left(\begin{array}{c}
H_{u}^{+} \\
H_{d}^{-*}
\end{array}\right) & =R_{\beta \pm}\left(\begin{array}{c}
G^{+} \\
H^{+}
\end{array}\right)
\end{aligned}
$$

with orthogonal rotation matrices $R_{\alpha}, R_{\beta 0}$ and $R_{\beta \pm}$. The three massless Goldstone bosons $G^{0}$ and $G^{ \pm}$are absorbed in the massive electroweak gauge bosons as in the SM. The remaining five degrees of freedom are two massive, CP-even Higgs bosons, $h^{0}$ and $H^{0}$, one CP-odd Higgs boson $A^{0}$ and two charged Higgs bosons, $H^{ \pm}$, the tree-level masses of which are given by [8]

$$
\begin{aligned}
m_{h^{0}}^{2} & =\frac{1}{2}\left(m_{A^{0}}^{2}+m_{Z}^{2}-\sqrt{\left(m_{A^{0}}^{2}-m_{Z}^{2}\right)^{2}+4 m_{Z}^{2} m_{A^{0}}^{2} \sin ^{2}(2 \beta)}\right) \\
m_{H^{0}}^{2} & =\frac{1}{2}\left(m_{A^{0}}^{2}+m_{Z}^{2}+\sqrt{\left(m_{A^{0}}^{2}-m_{Z}^{2}\right)^{2}+4 m_{Z}^{2} m_{A^{0}}^{2} \sin ^{2}(2 \beta)}\right), \\
m_{A^{0}}^{2} & =2 b / \sin (2 \beta), \\
m_{H^{ \pm}}^{2} & =m_{A^{0}}^{2}+m_{W}^{2} .
\end{aligned}
$$

While from equation (2.61) it follows that in the MSSM the tree-level mass of the light CP-even Higgs boson $h^{0}$ has an upper bound at

$$
m_{h^{0}}<m_{Z}|\cos (2 \beta)|<m_{Z},
$$

$m_{h^{0}}$ can receive significant corrections from fermion and sfermion loops that are typically of the form

$$
\Delta m_{h^{0}}^{2} \propto y_{i}^{2} \ln \left(\frac{m_{\tilde{f}_{i 1}} m_{\tilde{f}_{i 2}}}{m_{f_{i}}^{2}}\right)+\text { similar terms, }
$$

such that mainly due to the large value of $y_{\mathrm{t}}$ the mass of the lightest Higgs boson in the MSSM can achieve larger values - in particular, the mass of the resonance recently found at the LHC at $\sim 126 \mathrm{GeV}[6,7]$ can be achieved in the MSSM if the squark and slepton masses are not much larger than several $\mathrm{TeV}$. 
Gauginos and Higgsinos Due to electroweak symmetry breaking, the superpartners of the neutral Higgs gauge eigenstates mix with the superpartners of the neutral gauge eigenstates of the electroweak gauge bosons to form the mass eigenstates, which are called neutralinos. These mass eigenstates are obtained by diagonalising the neutralino mass matrix $\mathbf{M}_{\tilde{\chi}^{0}}$,

$$
\left(\mathbf{N}^{*} \mathbf{M}_{\tilde{\chi}^{0}} \mathbf{N}^{-1}\right)_{i j}=\delta_{j}^{i} m_{\tilde{\chi}_{i}^{0}},
$$

where $\mathbf{N}$ is the (unitary) neutralino mixing matrix. There are four neutralino mass eigenstates, each a mixture of the two neutral Higgsinos, the neutral Wino and the Bino, which are usually described by the symbols $\tilde{\chi}_{i}^{0}$. The neutralino mass matrix is typically given in the basis of gauge eigenstates $\left(\tilde{B}^{0}, \tilde{W}^{0}, \tilde{H}_{d}^{0}, \tilde{H}_{u}^{0}\right)$. The mass eigenstates are just ordered by increasing mass, where $i=1(i=4)$ denotes the lightest (heaviest) neutralino. In terms of the MSSM parameters $M_{1}$, $M_{2}, \mu$ and the angle $\beta$, the neutralino mass matrix at tree-level is given by

$$
\mathbf{M}_{\tilde{\chi}^{0}}=\left(\begin{array}{cccc}
M_{1} & 0 & -\cos \beta \sin \theta_{W} m_{Z} & \sin \beta \cos \theta_{W} m_{Z} \\
0 & M_{2} & \cos \beta \cos \theta_{W} m_{Z} & -\sin \beta \cos \theta_{W} m_{Z} \\
-\cos \beta \sin \theta_{W} m_{Z} & \cos \beta \cos \theta_{W} m_{Z} & 0 & -\mu \\
\sin \beta \sin \theta_{W} m_{Z} & -\sin \beta \cos \theta_{W} m_{Z} & -\mu & 0
\end{array}\right)
$$

with the weak mixing angle $\theta_{W}$. In this basis,

$$
\left(\begin{array}{c}
\tilde{\chi}_{1}^{0} \\
\tilde{\chi}_{2}^{0} \\
\tilde{\chi}_{3}^{0} \\
\tilde{\chi}_{4}^{0}
\end{array}\right)=\mathbf{N} \cdot\left(\begin{array}{c}
\tilde{B}^{0} \\
\tilde{W}^{0} \\
\tilde{H}_{d}^{0} \\
\tilde{H}_{u}^{0}
\end{array}\right)
$$

In order to avoid extreme CP-violating effects, a widely used convention is to have $M_{1}, M_{2} \in \mathbf{R}^{+}$ and $\mu \in \mathbf{R}$, although such effects can in principle also be cancelled by trilinear couplings (see, for instance [18]). The mixing matrix $\mathbf{N}$ plays a key role in the production of neutralinos at colliders as well as in their decay modes. This is detailed later in this section.

Similar to the neutral Higgsinos and the neutral gauginos, the superpartners of the charged Higgs and gauge bosons mix. The mass eigenstates are called charginos and are usually described by the symbols $\tilde{\chi}_{j}^{ \pm}$, where $j=1,2$ and $j=1$ denotes again the eigenstate with the smaller mass. By convention, the basis $\left(\tilde{W}^{+}, \tilde{H}_{u}^{+}\right)$for the positively charged charginos, and the basis $\left(\tilde{W}^{-}, \tilde{H}_{d}^{-}\right)$for the negatively charged charginos is used. The positively charged mass eigenstates are obtained by diagonalising the matrix

$$
\mathbf{X}^{T}=\left(\begin{array}{cc}
M_{2} & \sqrt{2} \cos \beta m_{W} \\
\sqrt{2} \sin \beta m_{W} & \mu
\end{array}\right)
$$

whereas the negatively charged mass eigenstates are obtained by diagonalising $\mathbf{X}$. Therefore, two different unitary matrices, usually referred to as $\mathbf{U}$ and $\mathbf{V}$ are needed to get the charginos, where

$$
\left(\begin{array}{c}
\tilde{\chi}_{1}^{+} \\
\tilde{\chi}_{2}^{+}
\end{array}\right)=\mathbf{V}\left(\begin{array}{c}
\tilde{W}^{+} \\
\tilde{H}_{u}^{+}
\end{array}\right), \quad \text { and } \quad\left(\begin{array}{c}
\tilde{\chi}_{1}^{-} \\
\tilde{\chi}_{2}^{-}
\end{array}\right)=\mathbf{U}\left(\begin{array}{c}
\tilde{W}^{-} \\
\tilde{H}_{d}^{-}
\end{array}\right)
$$

It can easily be shown that the two mass eigenvalues for the negatively charged mass eigenstates are the same as for the positively charged ones, such that

$$
\mathbf{U}^{*} \mathbf{X}^{T} \mathbf{V}^{-1}=\left(\mathbf{V}^{*} \mathbf{X} \mathbf{U}^{\dagger}\right)^{T}=\delta_{i}^{j} m_{\tilde{\chi} \pm_{j}} .
$$


Since gluinos do not mix with any other particle in the MSSM, the tree-level mass of the gluino is given by the mass parameter $M_{3}$,

$$
m_{\tilde{g}}=\left|M_{3}\right| .
$$

The implications of $M_{3}<0$ are sketched in [18].

Squarks and Sleptons Not only the superpartners of the gauge and Higgs bosons can mix, but in principle the same is also possible for squarks and sleptons. In that case, the mass eigenstates would be obtained by diagonalising a $6 \times 6$ matrix for each the up-type squarks, the down type squarks and the charged sleptons, and a $3 \times 3$ matrix for the sneutrinos. The masses of the squarks and sleptons are then determined by the explicit SUSY breaking terms involving the mass matrices, as well as by breaking electroweak symmetry and adding non-zero vevs to the Higgs fields. The latter effect arises from a) the F-term in the scalar potential (2.46), linear or quadratic in the Yukawa couplings $y_{i}$, and b) from the trilinear soft terms in (2.53), proportional to the trilinear couplings $a_{i}$. In addition, each squark and slepton mass receives a correction from the D-term in the scalar potential.

For most phenomenological models, mixing between families is assumed to be negligible for reasons already stated above. Secondly, most models assume that there is no mixing between the superpartners of the left chiral fermions and the superpartners of the right chiral fermions in the first two generations. Due to the large Yukawa couplings there, such an assumption is typically not valid for the third generation. Then, the mass eigenstates in the third family are indicated by an index $k=1,2$, where in the case of top-squarks for instance

$$
\left(\begin{array}{l}
\tilde{t}_{1} \\
\tilde{t}_{2}
\end{array}\right)=\mathbf{N}_{\tilde{t}} \cdot\left(\begin{array}{c}
\tilde{t}_{L} \\
\tilde{t}_{R}
\end{array}\right)
$$

where $\mathbf{N}_{\tilde{t}}$ is a unitary matrix. Examples for sfermion mixing and mass matrices can be found in [8], for instance.

\section{Collider Production and Decays of Superpartners in the MSSM}

If the physical masses of the superpartners are not too large, they can be produced at the LHC, for instance, or also at a future Linear Collider. There are several distinct scenarios to be considered at the LHC, where in the case of $R$-parity conservation the superpartners will be produced in even numbers. In the remainder of this section it is usually assumed that the lightest neutralino $\tilde{\chi}_{1}^{0}$ is the lightest supersymmetric particle (LSP). While a-priori the mass parameters in the Lagrangian can be chosen independently, other models are typically less interesting as they do not provide a good candidate for dark matter - this will be explained in more detail in the next section.

If for instance the masses of the squarks and/or gluinos are not too large, the dominant production mechanism at the LHC will be squark or gluino pair production, as well as the associate production of a squark and a gluino. Some tree-level Feynman diagrams for these processes are shown in Figure 2.4. While squarks can be produced via the strong as well as the electroweak interaction, due to the larger coupling constant the strong production will usually contribute dominantly to the scattering amplitude. While it is possible to construct models in which squarks are stable on lab scales, the average lifetime of squarks and gluinos is very small in most models. Depending on the MSSM mass hierarchy, several decays are allowed.

If the gluino is lighter than a squark, the decay $\tilde{q} \rightarrow \tilde{g} q$ is the dominant process. If the gluino is heavier than the squark, the modes $\tilde{q} \rightarrow \tilde{\chi}_{i}^{0} q$ and $\tilde{q} \rightarrow \tilde{\chi}_{j}^{ \pm} q^{\prime}$ become relevant. In the latter 


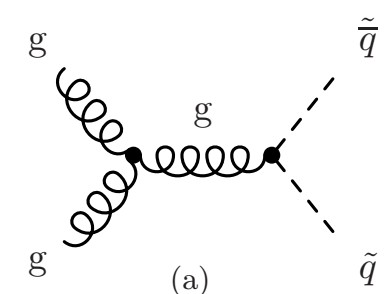

(a)

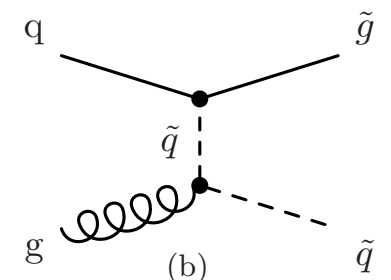

(b)

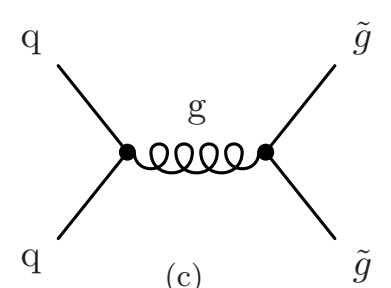

(c)

Figure 2.4: Three tree-level Feynman diagrams for the production of (a) squark pairs, (b) a squark in association with a gluino and (c) gluino pairs

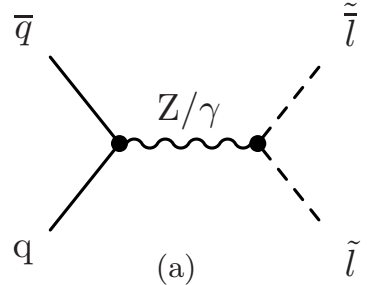

(a)

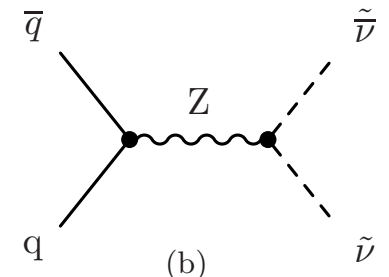

(b)

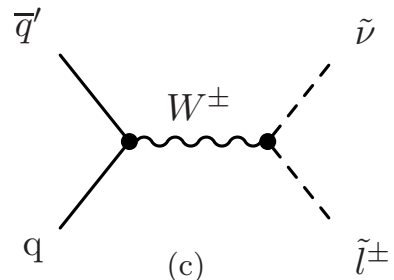

(c)

Figure 2.5: Three tree-level Feynman diagrams for the production of (a) charged slepton pairs, (b) sneutrino pairs, and (c) associate production of a sneutrino and a charged slepton.

case the relative branching fractions depend heavily on both the mixing in the squark sector as well as the mixing in the neutralino/chargino sector. The left chiral part of a squark mass eigenstate couples dominantly to a quark and the Wino components of the neutralino/chargino mass eigenstates, whereas the right chiral part couples mainly to the Bino component of the neutralino. The couplings to the Higgsino components are usually much smaller in the first and second generation, but can become significant for the third generation due to the large Yukawa couplings.

Gluinos can only decay via an on-shell or a virtual squark. If the gluino is heavier than the squark, the decay $\tilde{g} \rightarrow \tilde{q} q$ is by far the dominant contribution. If the gluino is lighter, it can decay via the three-body decay $\tilde{g} \rightarrow \tilde{\chi}_{i}^{0} q q$ and $\tilde{g} \rightarrow \tilde{\chi}_{j}^{ \pm} q^{\prime} q$. In both cases, it is again the mixing of the neutralinos/charginos and the squarks that determine the actual branching fractions.

If the superpartners of the coloured particles are too heavy, electroweak production of sleptons and neutralinos/charginos become the dominant production processes also at hadron colliders. Some tree-level diagrams for slepton pair production are shown in Figure 2.5.

In the case of slepton pair production, the mixing of the charged sleptons and the sneutrinos determines which production mechanism dominates. If kinematically allowed, sleptons decay via the two-body decays $\tilde{l} \rightarrow \tilde{\chi}_{i}^{0} l$ and $\tilde{l} \rightarrow \tilde{\chi}_{j}^{ \pm} l^{\prime}$. As in the case of squarks, the right chiral sleptons do not couple to the Wino components of the neutralinos/charginos, and the contributions due to Higgsino couplings are usually much smaller than the gauge coupling contributions, such that decays of right chiral sleptons into charginos are highly suppressed.

Depending on the mass spectrum, neutralinos and charginos are also dominantly produced via intermediate electroweak gauge bosons. In principle, any combination $\tilde{\chi}_{i} \tilde{\chi}_{j}$ of charginos and neutralinos can be produced. Associate production of a neutralino with a chargino happens mainly via the charged $W$ bosons in the s-channel. At a hadron collider, depending on the squark masses, the Bino component of the neutralino does only contribute via t-channel exchange of left chiral squarks. Associate chargino production and associate neutralino production mainly happens via a $Z$ boson in the s-channel as shown in Figure 2.6. Again there are contributions from t-channel diagrams which depend on the squark masses. Neutralinos couple to the $Z$ boson only due to their Higgsino components. There is no coupling between the Bino and Wino 

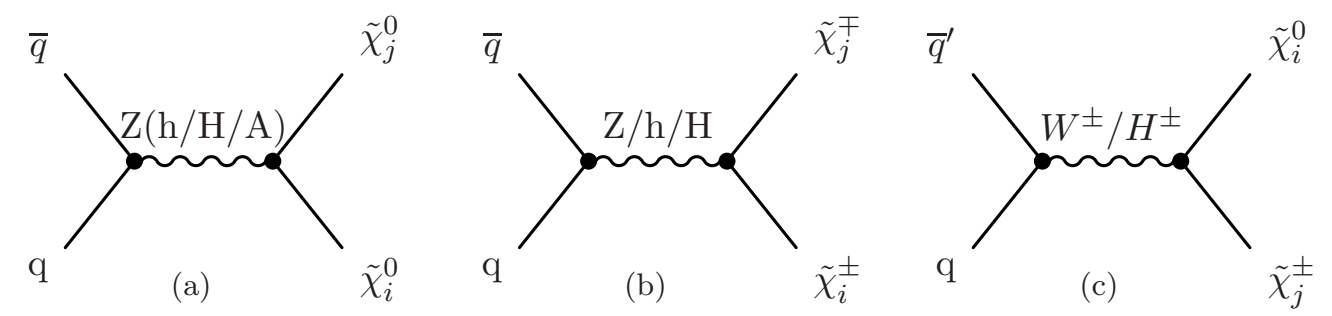

Figure 2.6: Three tree-level Feynman diagrams for the direct production of gauginos: The direct production of two neutralinos (a), the direct production of two charginos (b), and the associate production of a chargino with a neutralino (c).

component of a neutralino and a $Z$. This is because the $B^{\mu}$ has a weak hypercharge 0 such that it does not couple to the Bino-field, and $W_{0}^{\mu}$ does not couple to the neutral Winos due to the $S U(2)_{L}$ structure constants. As it will be of use later, the vertex factor for the neutralinoneutralino- $Z$ coupling as it appears in Figure 2.6(a), using the neutralino mixing matrix $\mathbf{N}$ is given by [48]

$$
g_{Z \tilde{\chi}_{i}^{0} \tilde{\chi}_{j}^{0}}=\frac{i e}{4 \sin \theta_{W} \cos \theta_{W}}\left(\left(N_{4 i}^{*} N_{4 j}-N_{3 i}^{*} N_{3 j}\right)\left(1-\gamma^{5}\right)-\left(N_{4 i} N_{4 j}^{*}-N_{3 i} N_{3 j}^{*}\right)\left(1+\gamma^{5}\right)\right) .
$$

There are additional s-channel diagrams that can in principle contribute to the neutralino pair production, namely via a Higgs boson. They can be of interest if the mass of that Higgs is larger than the sum of a Bino/Wino like neutralino $\tilde{\chi}_{i}^{0}$ and a Higgsino like neutralino $\tilde{\chi}_{j}^{0}$, in which case Bino/Wino-Higgsino pairs could as well be produced. Such production processes are however strongly suppressed.

Neutralinos and charginos have a large variety of decay modes. The branching fractions depend highly on the mass hierarchy and the mixing of the gauginos. The most important two-body decays of neutralinos and charginos are quoted here, taken from [8]:

$$
\begin{gathered}
\tilde{\chi}_{i}^{0} \rightarrow \tilde{\chi}_{j}^{0} Z / h^{0} / H^{0} / A^{0}, \tilde{\chi}_{j}^{ \pm} W / H^{\mp}, \tilde{f} f \\
\tilde{\chi}_{i}^{ \pm} \rightarrow \tilde{\chi}_{j}^{0} W^{ \pm} / H^{ \pm}, \tilde{\chi}_{j}^{ \pm} Z / h^{0} / H^{0} / A^{0}, \tilde{f} f^{\prime} .
\end{gathered}
$$

In particular for the light neutralinos/charginos, three-body decays may become relevant, where either one of the particles from the two-body decays is off-shell.

A more complete overview on the decays of supersymmetric particles is given in $[8,18]$.

\section{Low Energy SUSY Signatures and SUSY Dark Matter}

Apart from signals at high energy colliders, at which the superpartners of the SM particles may be produced directly, it is possible to look for indirect signals of SUSY - or physics beyond the SM in general - as well. In many cases these indirect signals arise due to supersymmetric versions of loop corrections, where the superpartners only appear as internal lines in the Feynman diagrams. If for instance there were large off-diagonal terms in the charged slepton mixing matrix, large contributions to flavour violating processes such as $\mu \rightarrow e$ would give a clear hint.

Similar observations could be made in the coloured sector. As an example, the process $b \rightarrow s \gamma$ is shown in Figure 2.7, to which supersymmetric diagrams can contribute. Experiments measuring such observables are usually performed at energies that are already now known to lie well below the soft SUSY mass scale $m_{\text {soft }}$. Thus, only off-shell superpartners are possibly involved in the interactions, and the respective observables are referred to as low energy observables (LEOs) in the following. 
It was already mentioned that the MSSM can provide an excellent candidate for dark matter. The standard model of cosmology typically strongly prefers cold dark matter, i.e. whatever particles dark matter is made of, they should not be relativistic. Usually this means that the masses of these particles must be relatively large, which rules out the SM neutrinos as dark matter candidates. Given a valid quantum field theory, the pair production and annihilation cross sections for dark matter particles can be calculated, i.e. rates for the process $\chi \chi \leftrightarrow$ $f f$, where $\chi$ is the dark matter particle. Using these cross-sections the amount of residual dark matter after cosmological freeze-out can be calculated, usually expressed by a quantity called dark matter relic density, $\Omega_{\mathrm{CDM}}$; see, for instance $[49,50]$. Although there are certain astrophysical objects like black holes or brown dwarves, that can account for a certain fraction of $\Omega_{\mathrm{CDM}}$, an alternative is needed. It can be shown that a massive, weakly interacting particle (WIMP) with a mass of $\sim 100 \mathrm{GeV}$ gives a relic density that is roughly compatible with current observations [51]. In the MSSM, there are in principle two types of WIMPs, the sneutrinos and the neutralinos.

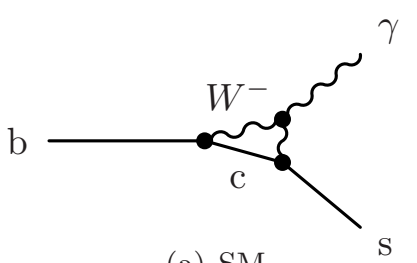

(a) SM

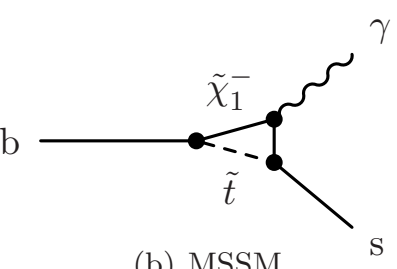

(b) MSSM

Figure 2.7: Two different diagrams contributing to the process $b \rightarrow s \gamma$.

Sneutrinos are generally less favoured as dark matter candidates, if SUSY is realised at the TeV scale, see for instance [52]. This is mainly due to the results of direct dark matter searches [53, $54,55,56,57,58]$. In some of these experiments the cross-sections for dark matter scattering off nucleons is measured, which dominantly happens by exchange of a $Z$ boson. While the coupling constant in the vertex factors for sneutrino- $Z$ couplings is the same as that for neutralino$Z$ couplings, the vertex factor (2.75) contains the difference of the products of the Higgsinocomponents only. If dark matter is mainly made of the lightest neutralino, and if the lightest neutralino is mainly Bino or Wino, the direct searches will not yield large signals. The lightest neutralino however would make a nearly perfect dark matter candidate, if its mass lay somewhere between $100 \mathrm{GeV}$ and a few $\mathrm{TeV}$. A detailed analysis of supersymmetric dark matter is given in [51].

\subsubsection{Simplified SUSY Breaking Models}

After some general aspects of the MSSM phenomenology have been described, this section deals with models that assume a certain mechanism for how SUSY breaking is mediated to the visible sector in Figure 2.3 and how the soft SUSY breaking terms in the MSSM arise. Then, the soft breaking terms are not completely arbitrary any more but they can be inferred from the breaking mechanism. Several constraints are imposed, such that a minimal version of each model is constructed. These minimal models usually are described by less than $\sim 10$ parameters. Nevertheless, since the communication between the MSSM and the hidden SUSY breaking sector is assumed to happen at some very high energy scales, the $\mathrm{TeV}$ scale implications of such models are expressed in terms of the soft SUSY breaking parameters (2.53), such that the phenomenology of these models can be deduced from the general MSSM phenomenology as described above. As it is important for the remainder of this thesis, the mSUGRA and closely 
related models are explained in a bit detail. The other candidates are only roughly sketched here.

\section{mSUGRA/CMSSM and NUHM $i$}

Making SUSY a local symmetry forces the introduction of a new gauge field, as it is the case for the known internal symmetries of the SM. The new gauge field is the graviton field that is introduced in a supermultiplet with its superpartner, the gravitino. This procedure yields a theory of gravity called supergravity. As other models for a quantum theory of gravity, supergravity is not renormalisable. Spontaneous breaking of local SUSY is achieved by giving a vacuum expectation value to an F-term in the scalar potential (2.46). The breaking of SUSY is then mediated from the hidden sector to the MSSM sector by gravitational interactions, by which the non-renormalisable terms in the Lagrangian density are suppressed by the Planck Mass $M_{P}$, such that these terms do not contribute to the phenomenology at low scales.

By assuming the unification of several of the MSSM parameters at the GUT scale $Q_{\mathrm{GUT}}$, a minimal supergravity scenario can be constructed, which is described by four continous parameters, $M_{1 / 2}, M_{0}, A_{0}$ and $\tan \beta$, and one discrete parameter which is the phase of $\mu$ and can have values -1 and +1 . All MSSM parameters are then expressed in terms of these five parameters:

$$
\begin{array}{rlr}
M_{3}=M_{2}=M_{1} & =M_{1 / 2} & \text { at GUT scale } \\
\mathbf{m}_{\tilde{\mathbf{Q}}}^{2}=\mathbf{m}_{\tilde{\mathbf{u}}}^{2}=\mathbf{m}_{\tilde{\mathbf{d}}}^{2}=\mathbf{m}_{\tilde{\mathbf{L}}}^{2}=\mathbf{m}_{\tilde{\mathbf{e}}}^{2} & =M_{0}^{2} \mathbf{1} & \text { at GUT scale } \\
\mathbf{a}_{\mathbf{u}}=A_{0} \mathbf{y}_{\mathbf{u}}, \mathbf{a}_{\mathbf{d}}=A_{0} \mathbf{y}_{\mathbf{d}}, \mathbf{a}_{\mathbf{e}} & =A_{0} \mathbf{y}_{\mathbf{e}} & \text { at GUT scale }
\end{array}
$$

in which $\mathbf{1}$ is the unit matrix and $\mathbf{y}_{\mathbf{i}}$ are the Yukawa matrices from the superpotential (2.47). Furthermore

$$
m_{H_{u}}^{2}=m_{H_{d}}^{2}=m_{0}^{2} . \quad \text { at GUT scale. }
$$

This model is referred to as minimal Supergravity (mSUGRA), or Constrained Minimal Supersymmetric Standard Model (CMSSM).

The unification of several parameters at the GUT scale is not forced by supergravity. Therefore it is easily possible to relax some of the boundary conditions (2.78) to (2.81). A popular choice for such a relaxation is to remove either the last or both equalities in equation (2.81) from the definition of the model, introducing one or two new parameters. These models are called Non-Universal Higgs Models (NUHM). In the case of $m_{H_{u}}^{2}=m_{H_{d}}^{2}$, that model is referred to as NUHM1, if the latter equation is not required to hold at the GUT scale, that model is referred to as NUHM2.

In the mSUGRA model, the masses of all sfermions and the masses of all gauginos are highly correlated at the GUT scale. As the particles involved belong to different representations of the gauge groups, the relations (2.78) to (2.81) do not hold at the electroweak scale $Q_{E W S B}$ due to loop corrections. The most general form for the physical sfermion mass at $Q_{E W S B}$ is then given by

$$
m_{\tilde{f}_{i}}^{2}=M_{0}^{2}+\kappa_{1}^{i} K_{1}+\kappa_{2}^{i} K_{2}+\kappa_{3}^{i} K_{3}
$$

where the coefficients $\kappa_{j}^{i}$ depend on the gauge quantum numbers of the fermion, and at one-loop level the $K_{j}$ are positive numbers with $K_{j} \sim m_{1 / 2}^{2}$. As the slepton masses do not receive loop corrections due to strong interactions, i.e. $\kappa_{3}^{\tilde{l}}=0$, the squarks are in general heavier then the sleptons. Also, as the right chiral sleptons do not interact weakly, the masses of the left chiral 
sleptons are larger. For the third generation, the calculation is complicated due to the finite Yukawa couplings which also contribute to the $\beta$-functions. The LSP in such models is usually either the lightest neutralino, or the light stau.

\section{Other Models}

One of the more popular alternatives to mSUGRA is a model in which the hidden sector communicates with the MSSM sector via both gravitational and also SM gauge interactions. The gauge interactions are much stronger than the gravitational interactions and become the dominant mediator of SUSY breaking. A new left chiral supermultiplet is introduced, the particles in which have a very high mass and couple to both the origin of SUSY breaking in the hidden sector by some interaction, as well as to the MSSM particles via the SM gauge interactions. Such models are called Gauge Mediated Supersymmetry Breaking (GMSB) models, in which as for mSUGRA F-type SUSY breaking appears. In GMSB models the LSP is usually the gravitino, which in final states at collider experiments appears in association with a photon, as usually the next to lightest supersymmetric particle (NLSP) decays into a gravitino and a photon.

In another large set of SUSY breaking models one or more additional space dimensions are introduced, similar to the UED models. SUSY breaking is assumed to be confined to the extra dimension (s), whereas the MSSM supermultiplets are mostly confined to the known fourdimensional space time. There are several ways how SUSY breaking is mediated to the MSSM sector, e.g. by the gauge supermultiplets or by supergravity entirely. If the SUSY breaking is mediated by supergravity exclusively, the models are referred to as Anomaly Mediated SUSY Breaking (AMSB) models. These models usually predict a nearly mass degenerate lightest chargino and neutralino.

\subsubsection{Fine-Tuning}

Searches for SUSY usually target regions in the MSSM where at least some of the physical masses of the superpartners are small. Apart from the fact that at contemporary colliders the prospects for finding evidence of SUSY with $m_{\text {soft }} \gtrsim 10 \mathrm{TeV}$ are negligible, a-priori there is no reason to believe that the superpartners in the MSSM should be light. There is however an aesthetical argument for this. In the SM, assuming that there is no new physics below some very large scale $Q_{\mathrm{GUT}}$, the Higgs mass depends heavily on parameters that are introduced at that scale. As there is then no direct relation between the electroweak scale and the scale $Q_{\mathrm{GUT}}$, a Higgs mass of the order of the electroweak scale is regarded to be unnatural. Ignoring for a moment the fact that it cannot provide electroweak symmetry breaking, in an unbroken supersymmetric theory this fine-tuning problem of the SM was solved, as sketched before. Even if SUSY is broken, if the masses of the superpartners are not too large, a Higgs mass of $\sim 100 \mathrm{GeV}$ can still be considered to be natural. This is what motivates $m_{\mathrm{SUSY}} \lesssim 10 \mathrm{TeV}$.

There is a certain amount of arbitrariness in the choice of the upper bound on $m_{\text {SUSY }}$ based solely on naturalness of the Higgs mass. Apart from the Higgs mass, there are other observables that can be fine-tuned, and a variety of measures for fine-tuning was defined. One popular measure according to $[59,60]$ is briefly described here.

Any observable $O$ can be expressed in terms of the model parameters, $a_{i}$, such that $O=O\left(a_{i}\right)$. The fine-tuning of that observable is then measured by the parameter $\Delta[O]$, which is defined by the scale independent derivatives

$$
c\left(a_{i}\right)[O]=\left|\frac{a_{i}}{O^{2}} \frac{\partial O^{2}\left(a_{i}\right)}{\partial a_{i}}\right| \quad \text { and } \Delta[O]=\max _{i}\left\{c\left(a_{i}\right)[O]\right\}
$$


In the remainder of this thesis, as it is a common choice to quantify the amount of fine-tuning for a SUSY model, $O=m_{Z}$ and the argument $O$ will be omitted. From upper bounds on $\Delta$ upper bounds on the SUSY mass scale can be inferred by requiring $\Delta<10,100,1000$ for instance. However, it must be emphasised that strictly speaking the choice of the upper bounds on $\Delta$ are completely arbitrary, as there is no physical answer to the question how much fine-tuning is acceptable and at which point a theory is unnatural - particularly in view of the need for SUSY breaking in order to give rise to a non-zero Higgs vev, and therefore yield $m_{Z} \neq 0$. 



\section{The Large Hadron Collider and the ATLAS Experiment}

CERN (Counseil Européenne pour la Recherche Nucléaire) is the European Organization for Nuclear Research with its main site located near Geneva at the border of Switzerland and France. Amongst the widespread scientific agenda at CERN there is the Large Hadron Collider and the associate particle detectors, the largest of which are ALICE, ATLAS, CMS and LHCb.

This chapter provides a brief overview of the LHC and the ATLAS experiment. After summarising the main features of the LHC, the ATLAS detector is described in some detail.

\subsection{The Large Hadron Collider at CERN}

The Large Hadron Collider [61] is a circular proton-proton, proton-heavy ion and heavy ionheavy ion collider. It has a circumference of roughly $27 \mathrm{~km}$ and is located roughly $100 \mathrm{~m}$ underground at CERN. Its construction was approved in 1994 and started in 2000, after the Large Electron Positron Collider (LEP) had been shut down. Making use of LEP's infrastructure the LHC was accelerating protons for the first time in September 2008. The first proton-proton collisions took place roughly 14 months later as after only 9 days of running the machine was severely damaged in 2008 due to a faulty connection.

\subsubsection{Design and Experiments}

Before being injected into the LHC itself, the protons are run through a series of pre-accelerators. Hydrogen atoms are stripped off their electrons and are accelerated to $50 \mathrm{MeV}$ in the LINAC2. In the Proton Synchroton Booster the energy per proton is raised to $1.4 \mathrm{GeV}$, after which the protons are accelerated to an energy of $25 \mathrm{GeV}$ in the Proton Synchrotron. The last step in the chain of pre-accelerators is the Super Proton Synchroton, from which the protons are injected into the LHC at an energy of $450 \mathrm{GeV}$. The acceleration chain is shown in Figure 3.1.

Using radio frequency (RF) cavities, the LHC can accelerate protons to energies up to $7 \mathrm{TeV}$ by design. The proton trajectories are bent around the circle by superconducting cryodipole magnets which provide a magnetic field of $8.33 \mathrm{~T}$. These are made of $\mathrm{Nb}-\mathrm{Ti}$ and operate at a nominal temperature of $1.9 \mathrm{~K}$. The RF cavities and a number of multipole magnets focus the beam. The LHC has been designed to store and accelerate 2808 bunches of protons separated into a clockwise and a counter-clockwise beam, with roughly $10^{11}$ protons per bunch. At each of the eight interaction points, where the beams can be brought to collision, a bunch crossing can appear at the minimal distance of $25 \mathrm{~ns}$. It was estimated that on average there would be about 20 proton-proton interactions per bunch crossing, which would correspond to an instantaneous luminosity of $10^{34} \mathrm{~cm}^{-2} \mathrm{~s}^{-1}$. The total cross section for proton-proton collisions at a centre of mass energy of $14 \mathrm{TeV}$ was estimated to be 40 mbarn for elastic scattering and 60 mbarn for inelastic scattering.

The LHC was also designed to collide heavy ions. $\mathrm{Pb}^{82+}$ ions can be accelerated to energies up to $2.76 \mathrm{TeV} /$ nucleon. The maximum instantaneous luminosity in ion mode was estimated to 


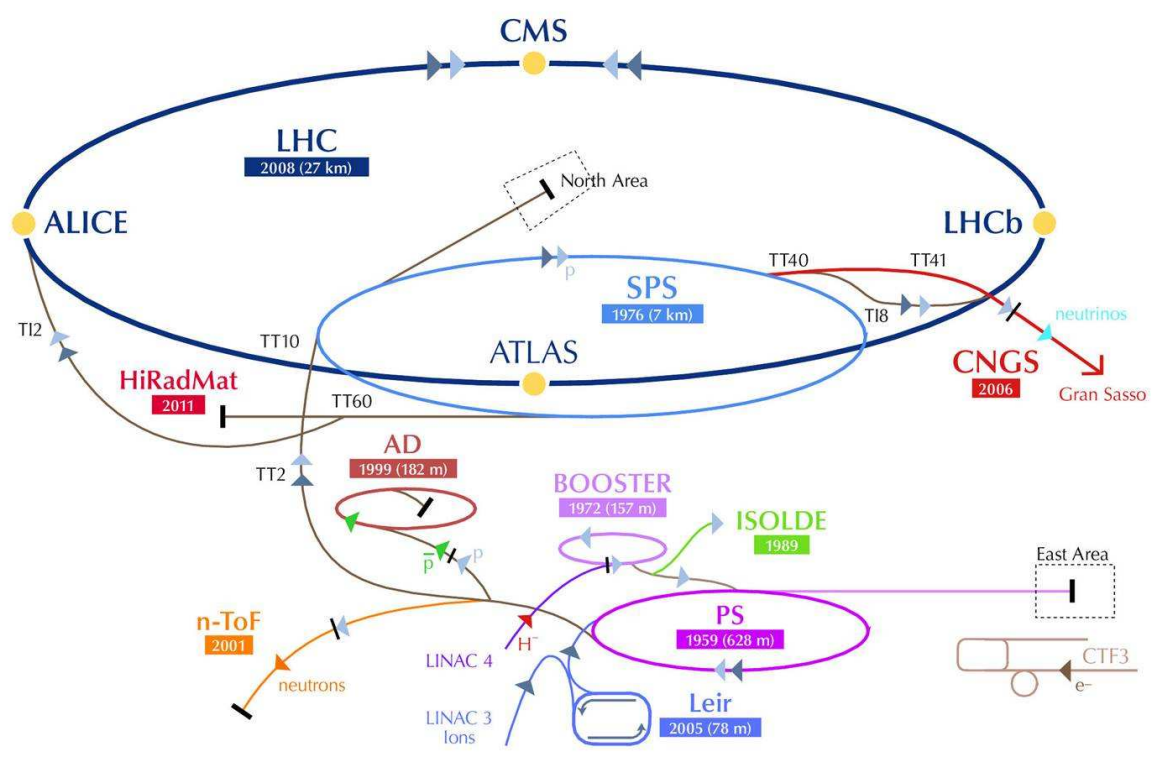

Figure 3.1: A schematic view of the CERN accelerator complex [62]. The chain of LHC preaccelerators is included: Starting at the LINAC2, the protons are accelerated by the Booster, the PS the SPS before they are finally injected into the LHC. The four large-scale experiments are also shown in the sketch.

be $7.0 \cdot 10^{7} \mathrm{~cm}^{-2} \mathrm{~s}^{-1}$, with 592 bunches of ions in the ring and a bunch spacing of $100 \mathrm{~ns}$.

With its design specifications, the LHC can provide high energy hadron collisions, raising the centre of mass energy $E_{C M}$ by a factor of roughly 7 compared to previous experiments. It is expected to cover a wide range of the parameter space of extensions to the SM as well as the SM itself, in particular the mass of the Higgs boson. In addition it provides the possibility to test the SM predictions in an energy regime that was not accessible under laboratory conditions so far. With the heavy ion collisions at even higher energies, it is also expected to provide new insights into the physics of the quark-gluon plasma.

There are two multipurpose detectors at the LHC, 'A Toroidal LHC Apparatus' (ATLAS) [63] and 'Compact Muon Solenoid' (CMS) [64]. Although using different technologies and putting emphasis on different aspects of particle detection, ATLAS and CMS have a similar structure and pursue a very similar physics program. Apart from the search for physics beyond the SM both experiments aim for precise measurements of SM physics, such as top-pair and single top production cross sections, the top quark properties, the search for the Higgs boson and measurement of its properties, or the measurement of production cross sections for electroweak bosons in association with jets. Particles are detected using an inner tracker, different calorimeters and muon chambers, as shown in Figure 3.2. For a more complete overview of techniques and methods for particle detection, see for instance [65, 66]. For the precise determination of particle momenta both experiments use sophisticated magnet systems. The ATLAS detector will be described in more detail in the second part of this chapter.

There are two more large scale experiments at the LHC with a focus on different physics questions. The LHCb detector [67] is a highly asymmetric detector with a rich agenda in $b$ physics, aiming at gaining insights into $\mathrm{CP}$ violation. With a focus on heavy ion physics, the ALICE detector [68] has been build to study the structure and behaviour of the quark-gluon plasma, mainly. 


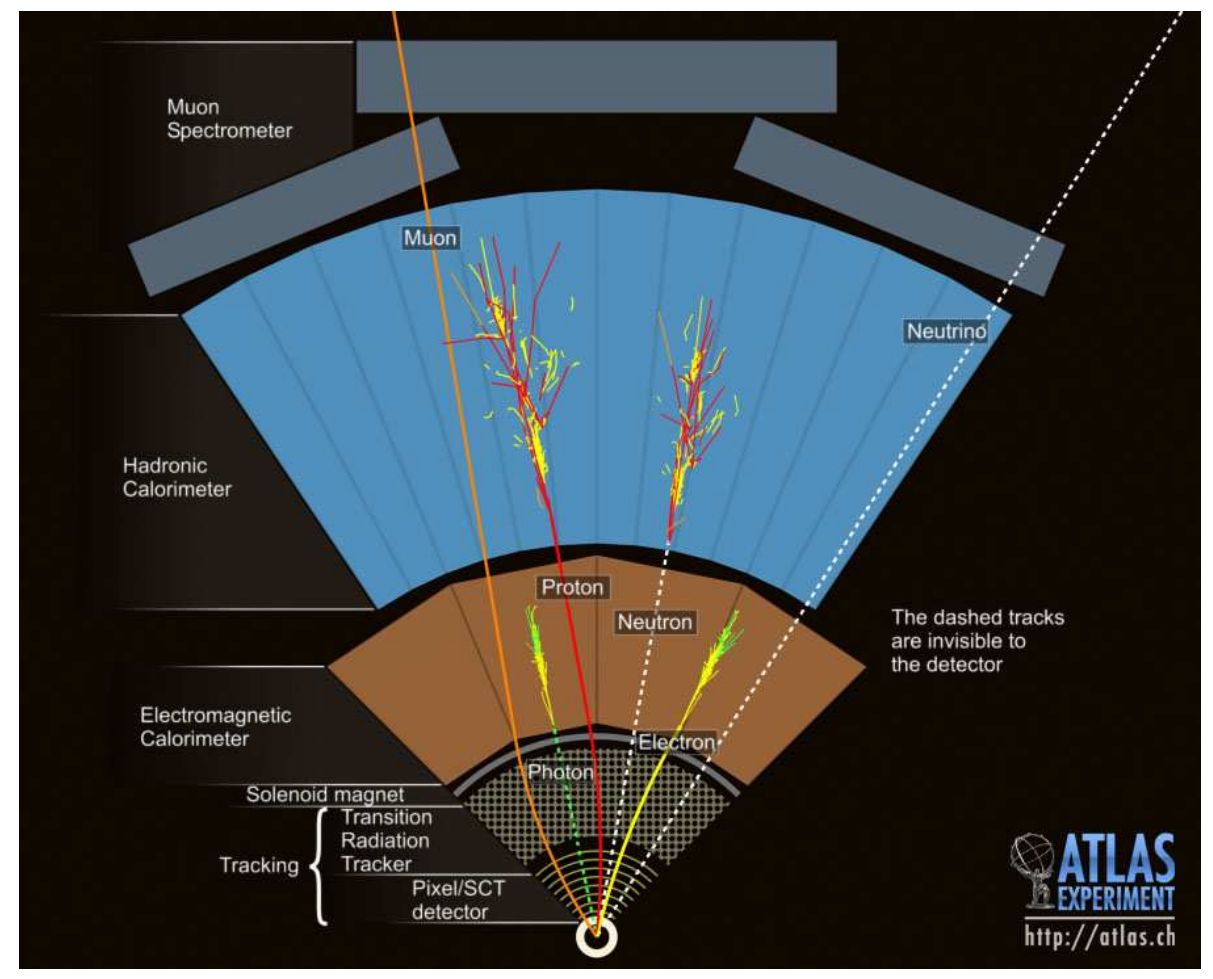

Figure 3.2: This schematic illustrates how the onion shell structure of modern multipurpose detectors allows for the reconstruction of nearly all known (semi)stable particles [69]. Dashed lines denote invisibility of particles in the respective detector parts. As shown, an electron leaves hits in the tracker and creates an electromagnetic shower in the electromagnetic calorimeter. If such a shower is found in the calorimeter without an associated track, it most likely originates from a photon. Charged hadrons can be identified by entries in the electromagnetic and hadronic calorimeters with an associated track in the inner detector, while neutral hadrons only deposit their energy in the hadronic calorimeter. This is exemplarily shown for a proton and a neutron, here. Muons, which are stable on the detector scale, are the only particles which are expected to leave tracks in the muon chambers. However, it may happen that the content particles of a jet are not fully stopped in the calorimeter and cause hits in the muon chambers, too (punch through). Of course, as shown here, muons can also be seen by the inner detector, such that for precise muon reconstruction the combined information of the inner tracker and the muon system may be used. The picture also shows a neutrino, which escapes the detector without leaving a significant amount of energy in the calorimeters, or hits in the tracking detectors.

In addition to the larger experiments, the LHC is used as an accelerator for smaller experiments as well. Located close to the ATLAS detector with the goal of improving hadron interaction models for cosmic ray studies there is the LHCf detector [70]. The TOTEM detector has the goal to measure the total proton-proton cross section using a luminosity independent method [71]. The MoEDAL experiment searches for magnetic monopoles and semi-stable massive particles [72].

\subsubsection{Operation in 2010-2012}

Operation in p-p mode in 2010 and 2011 After a short commissioning phase with beam energies of $450 \mathrm{GeV}$ and $1.05 \mathrm{TeV}$, the LHC ran at $3.5 \mathrm{TeV} /$ beam in 2010 and 2011. In 2010, a 
relatively small dataset was collected, resulting in a total of roughly $35 \mathrm{pb}^{-1}$ for physics analyses. The total luminosity could be measured with a final uncertainty of $3.4 \%$ in 2010 [73]. This data has not been used for the results presented in this thesis.

In 2011, the instantaneous luminosity could be significantly enhanced, yielding a dataset of about $5.25 \mathrm{fb}^{-1}$, as shown in Figure 3.3(a) with an uncertainty of 3.7\% [74]. The data is split into different periods during which the beam and detector conditions are roughly stable. A summary of the periods in 2011 is given in Table 3.1 .

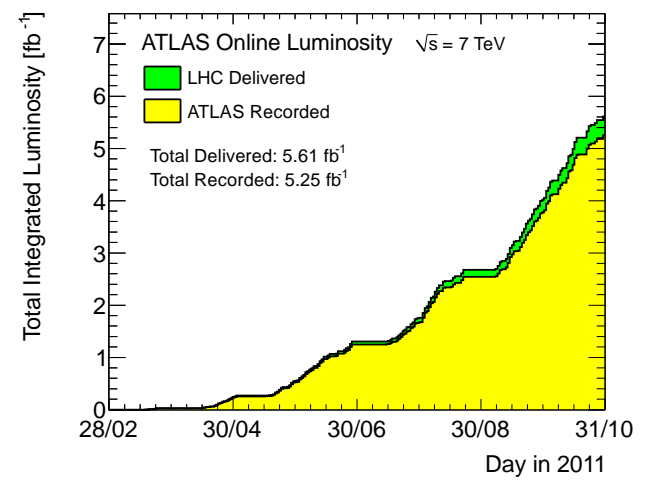

(a) 2011

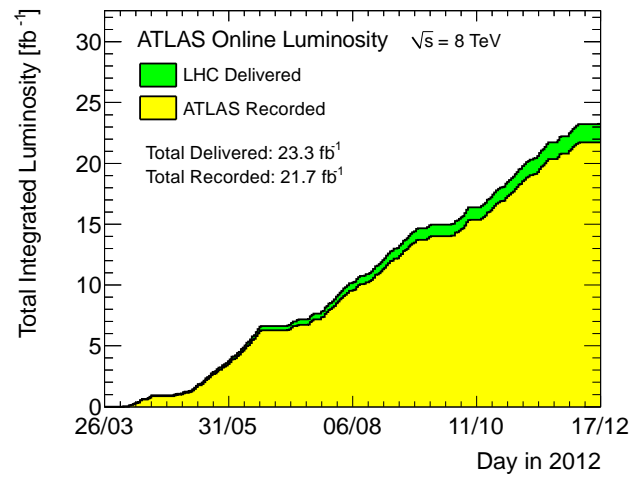

(b) 2012

Figure 3.3: Performance of the LHC and the ATLAS detector in 2011 and 2012

Operation in p-p mode in 2012 In 2012 the centre of mass energy was increased to $8 \mathrm{TeV}$, corresponding to a beam energy of $4 \mathrm{TeV}$. A total of $21.7 \mathrm{fb}^{-1}$ was collected, as shown in figure 3.3(b). The integrated luminosity could be determined with an uncertainty of $3.6 \%$. A summary of the periods in 2012 is also given in Table 3.1.

\begin{tabular}{c|c|c} 
period & 2011 integrated luminosity $\left[\mathrm{pb}^{-1}\right]$ & 2012 integrated luminosity $\left[\mathrm{pb}^{-1}\right]$ \\
\hline A & 9 & 910 \\
B & 18 & 5594 \\
C & - & 1643 \\
D & 186 & 3598 \\
E & 53 & 2863 \\
F & 160 & - \\
G & 572 & 1404 \\
H & 287 & 1655 \\
I & 416 & 1149 \\
J & 240 & 2941 \\
K & 685 & - \\
L & 1,625 & 983 \\
M & 1,184 & 14
\end{tabular}

Table 3.1: ATLAS data taking periods in 2011 and 2012 


\subsection{The ATLAS Experiment}

The ATLAS detector, located at Point 1 directly at the CERN main site, is a classical high energy physics multipurpose detector with a length of about $50 \mathrm{~m}$ and a diameter of roughly $22 \mathrm{~m}$. A schematic view of the detector is shown in Figure 3.4. Closest to the interaction point the inner detector is located. It consists of three subsystems: A silicon pixel detector, a silicon microstrip tracker and a transition radiation tracker. A solenoid magnet provides a magnetic field of roughly $2 \mathrm{~T}$. The electromagnetic calorimeter is a sampling calorimeter, using lead as an absorber material and liquid Argon as active material. The same technology is used for parts of the hadronic calorimeter, complemented by a hadronic tile calorimeter. The overall size and shape of the ATLAS detector is determined by the muon system, for which a toroidal magnet provides a magnetic field of up to $4.1 \mathrm{~T}$. The detector and its subsystems are usually divided into three parts - a central barrel part and an endcap on each side, such that the overall detector is roughly cylindrically symmetric.

The $\sim 100$ million readout channels produce an enormous amount of data per event, such that a three level trigger system is used to reduce the actual rate. Apart from so-called minimum-bias events, the trigger can be configured to select well defined final states based on combinations of three independent signatures (tracking, calorimetry, muon system). The raw data of events that have been selected for recording are stored and prepared for physics analyses.

\subsubsection{The ATLAS Coordinate System}

The origin of the ATLAS coordinate system is defined by the nominal interaction point at Point 1. The beam pipe defines the $z$-axis, the plane perpendicular to that axis is the $x-y$ axis. The positive $x$-axis points from the interaction point to the center of the LHC ring, the positive $y$-axis points upwards. The counter clockwise beam moves in the positive $z$-direction of the coordinate system.

The azimuthal angle $\phi \in[-\pi, \pi]$ is defined in the $x-y$ plane, with $\phi=0$ corresponding to

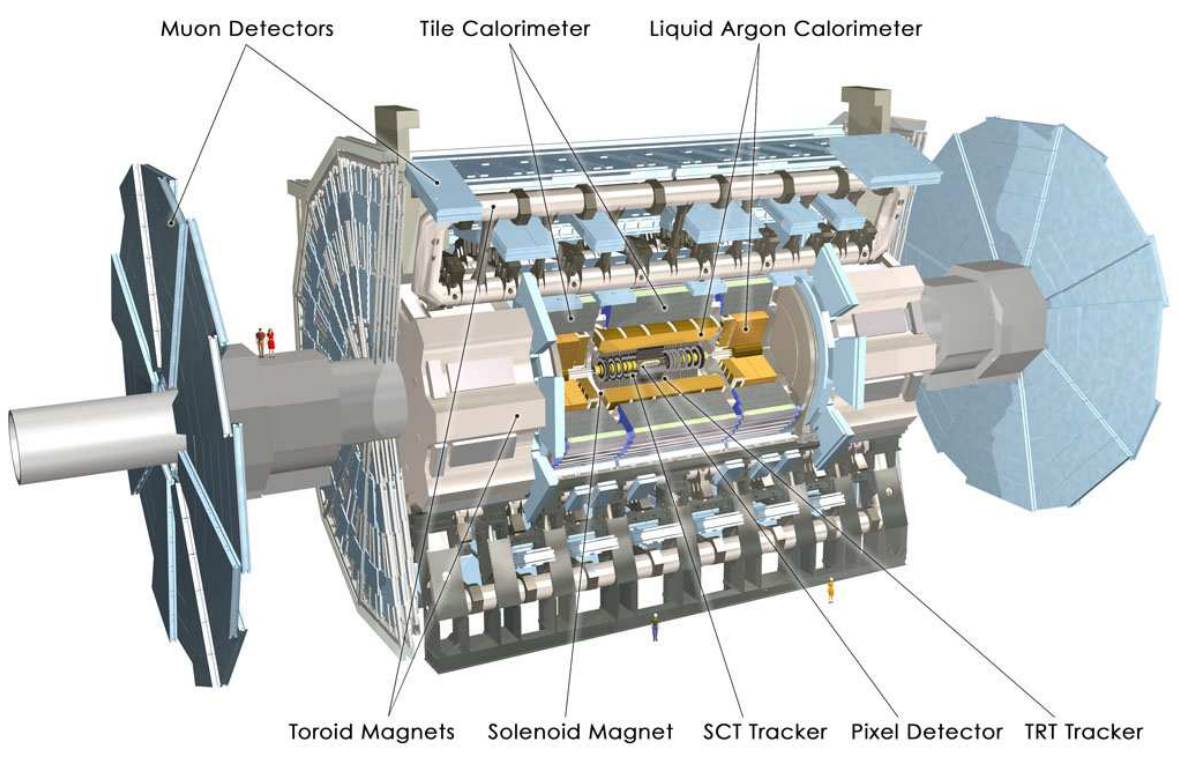

Figure 3.4: An overview of the ATLAS detector [69]. The main subsystems are labelled. The scale is illustrated by the two persons standing next to the very left muon detectors. 
the positive $x$-axis and $\phi=\pi / 2$ corresponding to the positive $y$-axis. Instead of the polar angle $\theta$ it is common to use the pseudorapidity $\eta=-\ln \tan \frac{\theta}{2}$ as differences in rapidity are Lorentz invariant and in the high energy limit this is equal to the pseudorapidity.

\subsubsection{The ATLAS Inner Detector}

The ATLAS inner detector (ID) $[75,76]$ consists of three independent subdetectors, covering all pseudorapidities $|\eta|<2.5$. It has a length of roughly $6 \mathrm{~m}$ and a diameter of roughly $2 \mathrm{~m}$. Charged particles interact with the detector material in this region, possibly creating a hit in each layer of the inner detector that can be used to reconstruct tracks. The solenoid provides a magnetic field of roughly $2 \mathrm{~T}$ such that the trajectories of charged particles are bent in the ID, which can be used to determine the particles' momentum. Using several tracks, interaction vertices can be reconstructed, which is useful for identifying $\tau$-leptons and heavy-flavour jets, for instance. A schematic of the ATLAS ID is shown in Figure 3.5.

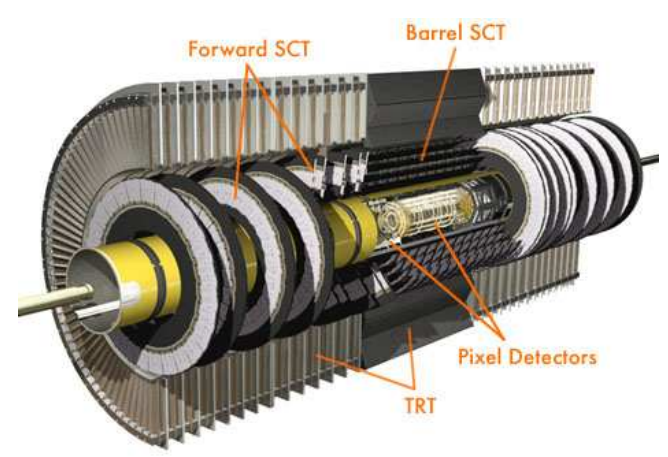

(a)

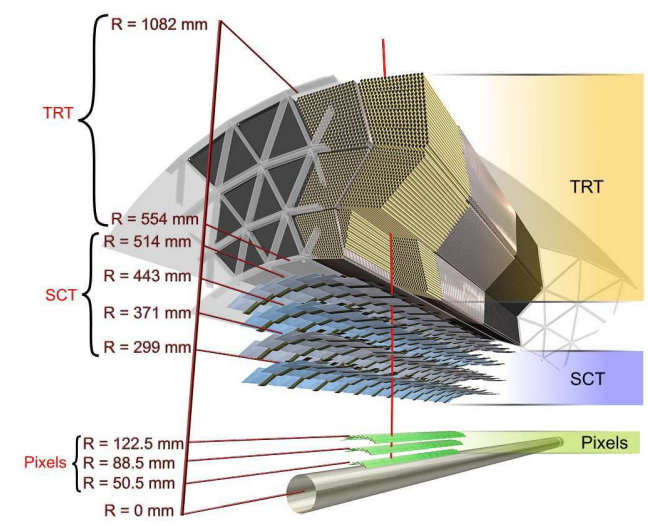

(b)

Figure 3.5: The ATLAS inner detector, which consists of the pixel detector, the semiconductor tracker and the transition radiation tracker [77]. (a) shows a longitudinal cross section of the ID, while (b) shows a transversal cross section and the radial distance of the separate detector parts from the beam pipe. From both sketches, the relative vicinity of the pixel detector to the beam pipe with respect to the other ID subsystems can be seen.

The Pixel Detector The pixel detector is located closest to the beam pipe and the nominal interaction point; of its three layers, the closest has a distance of only $5 \mathrm{~cm}$ to the beam pipe, the other layers are situated at a distance of about $8.9 \mathrm{~cm}$ and $12.3 \mathrm{~cm}$ from the beam pipe. The pixel sensors consist of $\mathrm{n}^{+}$-on- $\mathrm{n}$ doped silicon on which a bias voltage of $150 \mathrm{~V}$ is applied. Charged particles generate a signal by creating electron-hole pairs in the depleted material.

Of all subsystems in the ATLAS detector, the pixel detector has the highest granularity and by far the highest number of read-out channels, roughly 80 million. Depending on its position, each pixel has a width of $50 \mu \mathrm{m}$, a length of $400 \mu \mathrm{m}$ or $600 \mu \mathrm{m}$ and thickness of $250 \mu \mathrm{m}$. The sensor is bump-bonded to the read-out electronics.

By design, the pixel detector has a resolution of $12 \mu \mathrm{m}$ in $R \phi$ in both the barrel and the endcaps, and $66 \mu \mathrm{m}$ (barrel) or $77 \mu \mathrm{m}$ (endcaps) in $z$. Although the pixel detector provides an excellent coverage with small areas of dead material, due to the extreme radiation during LHC 
runs its performance degrades, such that parts of it will be replaced before the next long run of the LHC.

The Semiconductor Tracker Next to the pixel detector, at distances between $29.9 \mathrm{~cm}$ and $51.4 \mathrm{~cm}$ from the beam pipe, the Semiconductor Tracker (SCT) consists of several layers of silicon strips. One module contains four p-on-n silicon strip sensors. In the central region a pair of sensors is attached to each other at a relative angle of $40 \mathrm{mrad}$, where one of the strips is parallel to the beam pipe. The pitch between two strips is $80 \mu \mathrm{m}$. The modules in the endcaps are constructed in a similar way, but instead of a parallel alignment, one of the strips is aligned perpendicular to the beam pipe. The SCT has a design resolution of $16 \mu \mathrm{m}$ in $R \phi$ and $580 \mu \mathrm{m}$ in the $\mathrm{z}$ direction.

The Transition Radiation Tracker With a distance of $55.4 \mathrm{~cm}$ to $1.1 \mathrm{~m}$, the transition radiation tracker (TRT) is the outermost subsystem of the ID. The TRT is a gas tube detector, where a mixture of Carbon-dioxide, Tetrafluormethane and Xenon is used. Each tube, of which there are 50,000 in the barrel region and 320,000 in the endcaps, has a diameter of $4 \mathrm{~mm}$. Charged particles ionise the gas in the tubes, such that from a large number of hits tracks can be fitted. In addition, the TRT uses the characteristic transition radiation to identify and separate between different particles, most importantly between electrons and pions. There are two independent thresholds defined for each channel; a low threshold is used to identify hits, and a significantly larger threshold is used for the actual transition radiation. The number of hits in the TRT (typically more than 32 ) is usually much larger than the number of hits in the other subsystems of the ID.

\subsubsection{The ATLAS Calorimeters}

The ATLAS Calorimeters [78, 79] are used to measure the energy of particles and jets from quarks and gluons in an energy range of more than two orders of magnitude. Light charged particles and photons are stopped by Bremsstrahlung, pair creation and ionisation in the electromagnetic calorimeter which is located next to the solenoid providing the magnetic field for the ID. Particles interacting strongly with the nuclei of the detector material are stopped in the hadronic calorimeter next to the electromagnetic calorimeter. By measuring all the energy deposited in the calorimeters by electromagnetically and strongly interacting particles, it is also possible to deduce the existence of only weakly interacting particles, such as neutrinos, in events where there is an energy imbalance (missing transverse energy, $\mathbb{E}_{T}$ ).

\section{The Electromagnetic Calorimeter}

The electromagnetic calorimeter (ECAL) covers an pseudorapidity range of $|\eta|<3.2$. It is made of accordion shaped lead plates, the gaps between which are filled with liquid Argon as the active material. Copper electrodes are situated in the liquid Argon and a high voltage is applied to collect ionisation charges generated by electromagnetically interacting particles. The ECAL is divided into four parts, two barrel parts separated by a central gap of roughly $6 \mathrm{~mm}$ and covering the range $|\eta| \lesssim 1.5$, and two endcaps, covering $1.4<|\eta|<3.2$. With respect to the final state particles direction of movement, the ECAL is divided in both the longitudinal as well as the transverse direction, allowing a precise reconstruction of the electromagnetic shower and reconstructing for instance the momentum vector for photons. In order to avoid punch-through, the thickness of the ECAL exceeds 24 radiation lengths nearly everywhere. As 
particles do inhomogeneously loose energy in the ID, for $|\eta|<1.8$, the ECAL is supplemented by a presampler in order to measure the energy loss of particles before they reach the actual calorimeter. Apart from the presampler, there are three separate layers in the ECAL, both in the barrel and in the endcaps. The first layer provides a high granularity in order to disentangle neutral pions and single photons. The granularity gets coarser in the second layer, which contains the largest fraction of the electromagnetic shower, and the third layer that can be used to reject hadronic showers.

Depending on the layer and the position in the calorimeter, the ECAL cells cover an area from $0.003 \times 0.1$ up to $0.1 \times 0.1$ in terms of $\delta \eta \times \delta \phi$. In total, there are roughly 36, 000 channels in each endcap and about 106,000 channels in the barrel. The ECAL achieves a resolution of

$$
\frac{\Delta E}{E}=\frac{11 \%}{\sqrt{E[\mathrm{GeV}]}} \oplus 0.4 \% .
$$

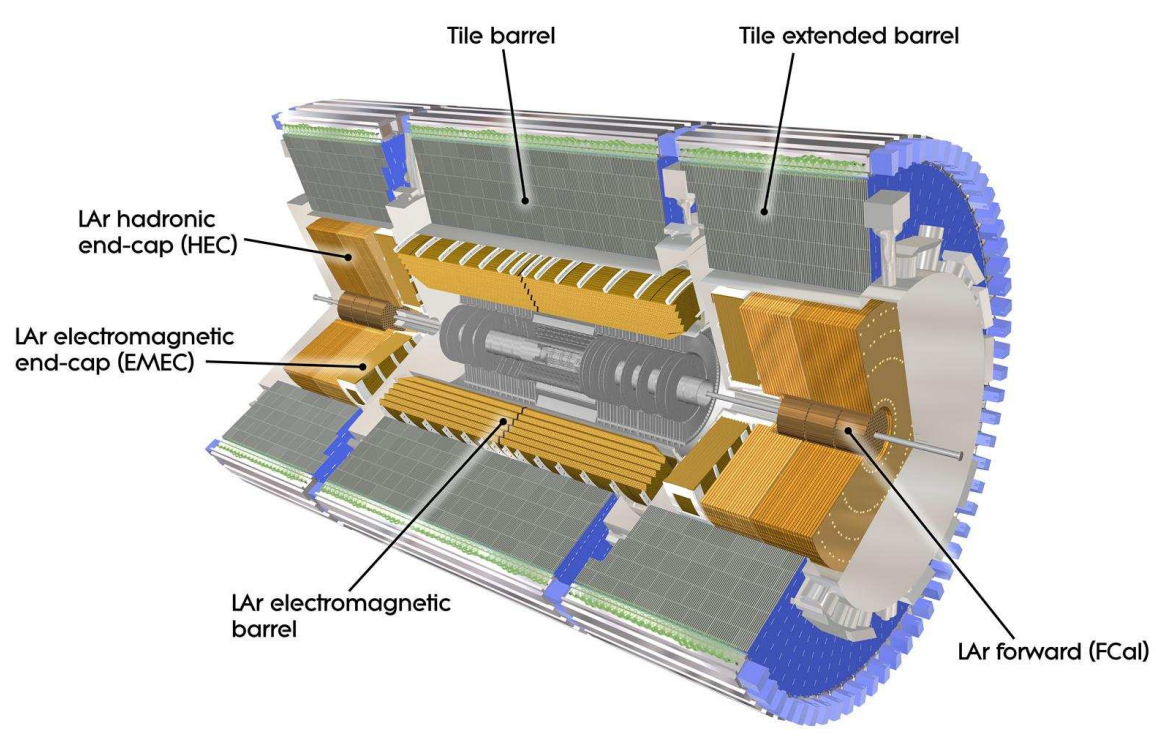

Figure 3.6: The ATLAS calorimeters [77]. The different parts of the calorimeter systems allowing for the measurement of the energies of electrically charged particles and hadrons are labelled.

\section{The Hadronic Calorimeters}

There are two different types of hadronic calorimeters in ATLAS. One type is based on the same technology as the ECAL. Covering a range of $1.5<|\eta|<3.2$, there are the hadronic endcaps (HEC). Instead of lead, Copper is used in these detectors as an absorber material, while as in the ECAL, liquid Argon is the active material. There are two HEC wheels per endcap with a thickness of 10 interactions lengths. The HCAL has a total of roughly 6, 200 read-out channels.

For the very forward regions, $3.2<|\eta|<4.9$, the forward calorimeter (FCAL) also uses ionisation of liquid Argon to measure both electromagnetic as well as hadronic showers. It consists of three wheels per endcap, the first of which has a thickness of 2.6 interaction lengths and uses Copper as an absorber. In the second and third wheel the absorber material is Tungsten, 
and both wheels have a thickness of about 3.7 interaction lengths each. There are roughly 3,600 read-out channels in the FCAL.

In the barrel, as well as in the outer parts of the endcaps, a hadronic tile calorimeter (TileCal) is used to measure hadronic showers. The barrel part covers the range $|\eta|<1.0$, while the tile extended barrel cover pseudorapidities of $0.8<|\eta|<1.7$. Scintillator tiles of polystyrene are used as an active material, while iron plates are used as absorbers. The TileCal is divided into three longitudinal layers with cells covering an area of $0.1 \times 0.1$ up to $0.2 \times 0.1$ in terms of $\delta \eta \times \delta \phi$ and a total thickness of more than 7 interaction lengths. The signals from the scintillating tiles are read out using wavelength shifters and photomultipliers.

\subsubsection{The ATLAS Muon Systems}

All known particles other than neutrinos and muons are likely to decay in or before they reach the ID, or are stopped in most cases in the calorimeters. While neutrinos escape the detector without producing hits, muons can be identified using another layer of tracking detectors installed behind the calorimeter. This outermost collection of subdetectors at the ATLAS detector is the muon system [80] as shown in Figure 3.7.

Three layers of muon chambers in the barrel and four layers in the endcaps are used in combination with a magnetic field provided by a system of three magnets. For $|\eta|<1.0$ this is generated by a barrel toroid. For the transition region, $1.0<|\eta|<1.4$, the magnetic field arises due to the central toroids as well as due to the endcap magnets. For $|\eta|>1.4$ the mangetic field is provided solely by the endcap magnets.

Four different technologies are used to identify muons, two of them for triggering on muons and two of them for precision measurements. In the barrel part as well as in all but the innermost endcap wheels, monitored drift tubes (MDT) are used for precision measurements. The MDTs consist of aluminium tubes which are $30 \mathrm{~mm}$ in diameter, $400 \mu \mathrm{m}$ thick and filled with an Argon/Carbon-dioxide mixture. Signals due to the ionisation of the gas are transmitted via a Tungsten wire. The length varies between $0.7 \mathrm{~m}$ and $6.3 \mathrm{~m}$. The MDTs achieve a resolution of $80 \mu \mathrm{m}$ per tube and $35 \mu \mathrm{m}$ per chamber in the $r-\varphi$ direction and are used in the range $|\eta|<2$. The alignment is monitored by an in-plane optical system. There is a gap in the coverage at roughly $|\eta|<0.05$ due to the support structure for the solenoid magnet. Also in the lower half of the detector where $\phi<0$, the acceptance of the muon system is significantly decreased in two regions where the whole detector is mounted.

In order to meet the higher radiation rates in the more forward region (covering $2.0<|\eta|<$ 2.7), cathode strip chambers (CSC) are used. These are multiwire proportional chambers with a good spatial resolution (better than $60 \mu \mathrm{m}$ ) due to the segmentation of the read-out cathode and charge interpolation between neighbouring strips. Also a good timing resolution is achieved. A mixture of Argon, Carbon-dioxide and tetrafluormethane is used to generate the signal.

For fast read-out and allowing to trigger on muons, resistive plate chambers (RPC) are used in addition to the MDTs in the barrel. RPCs are made of a mixture of sulfur hexafluoride and tetrafluorethane filled in gaps between two parallel resistive bakelite plates. Two orthogonal rectangular layers allow for the measurement of two coordinates, $\eta$ and $\phi$. The RPCs cover the central region up to roughly $|\eta|<1.05$.

In the endcaps, thin gap chambers (TGC) are used to trigger effectively on muons with a pseudorapidity of up to $|\eta|<2.4$. The TGCs are made of two cathode plates at a distance of $2.8 \mathrm{~mm}$ from each other and an anode wire located in between the cathodes. A mixture of Carbon-dioxide and $\mathrm{C}_{5} \mathrm{H}_{10}$ is ionised. A small drift time ensures a good timing resolution and fast signal read-out is achieved. 


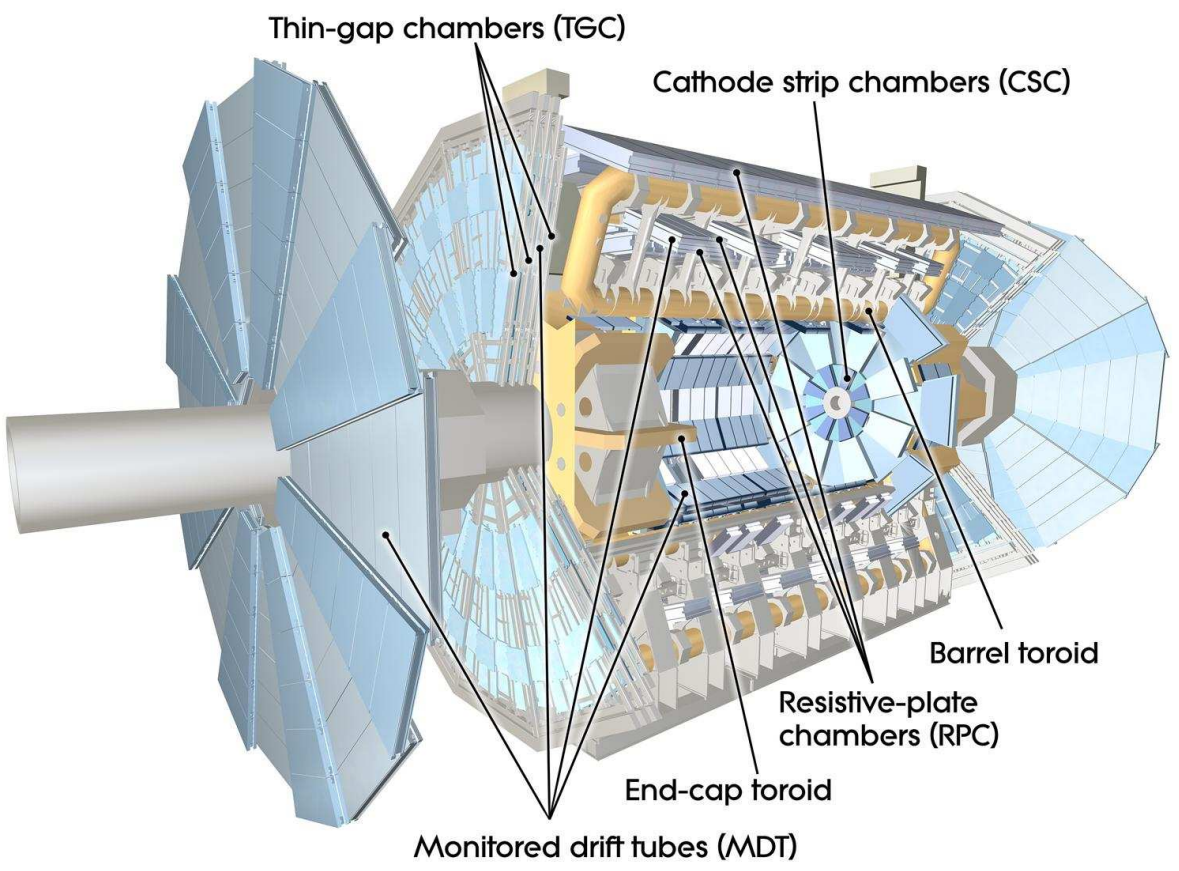

Figure 3.7: This is a scheme of the ATLAS muon systems [77]. The figure shows the arrangement of the CSCs, TGCs, RPCs and MDTs. Furthermore, the toroid magnets for the bending of the muon tracks are labelled.

\subsubsection{The ATLAS Magnet Systems}

In order to measure the momentum of charged particles, their trajectories are bent by a magnetic field in the tracking devices of the detector. The curvature depends on both the momentum $p$ of the particle as well as the magnitude $B$ of the magnetic field, such that the bending radius is approximately given by

$$
r[\mathrm{~m}]=\frac{p[\mathrm{GeV}]}{0.3 B[\mathrm{~T}]} .
$$

It can be inferred that the relative uncertainty on the track momentum is asymptotically linear to the track momentum itself. The stronger the magnetic field is, the better the resolution gets.

In ATLAS, the magnetic fields are provided by two magnets. One solenoid magnet located right next to the ID at radii $1.22 \mathrm{~m}<r<1.32 \mathrm{~m}$, generates a magnetic field of about $2 \mathrm{~T}$ for the ATLAS ID. With respect to the direction of motion of particles emerging from the hard interaction, the solenoid is placed in front of the calorimeters. While by this construction the need of having the magnet close to the ID does not limit the size of the calorimeter, it limits the thickness of the magnet in order to avoid large energy losses within it. The solenoid has a length of roughly $5.8 \mathrm{~m}$.

The magnetic field for the tracking chambers in the ATLAS muon system is provided by three independent air-core toroid magnets in order to keep the thickness of the magnet in terms of the average radiation length at a minimum. The central part consists of eight coils with a length of about $25 \mathrm{~m}$ each. The two endcap magnets are rotated by $22.5^{\circ}$ with respect to the central toroid in order to achieve the optimal bending power in the overlap region of the two magnets on each side. A magnetic field of $3.9 \mathrm{~T}$ in the central region and $4.1 \mathrm{~T}$ in the endcap regions is provided by the magnets. 
Both magnet systems are operated at a temperature of $4.5 \mathrm{~K}$ to ensure superconductivity. The magnetic field is generated by currents of roughly $7.7 \mathrm{kA}$ in the solenoid and roughly 20.4 $\mathrm{kA}$ in the toroids.

\subsubsection{The ATLAS Trigger System}

The enormous amount of data that the ATLAS detector produces can not be stored completely. More than $1 \mathrm{PByte} / \mathrm{s}$ would have to be written to disk, which is impossible with the available computing structure at the ATLAS experiment. In addition, far less than $1 \%$ of the events at the ATLAS interaction point are 'interesting' events. While there are numerous definitions of 'interesting', in general an event is considered to be such if for instance it contains high $p_{T}$ leptons and/or jets, or a certain number of these objects, or a certain amount of missing transverse energy. Such events are extremely rare and a dedicated system is needed to select them for further processing with a high efficiency and purity.

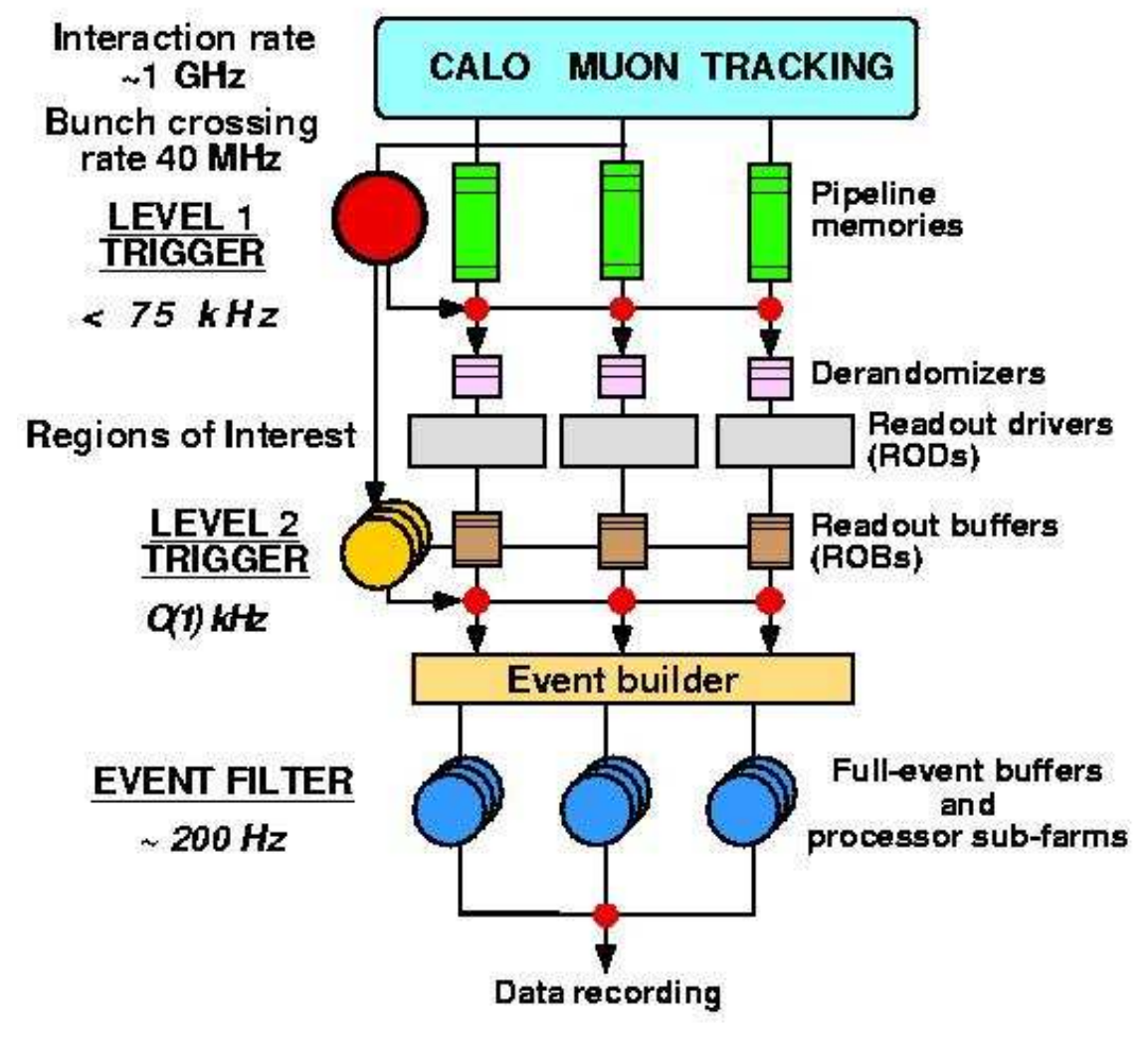

Figure 3.8: This is an illustration of the ATLAS trigger system [81]. The different stages of triggering and the individual components of each trigger are shown. On the left hand side, the respective input/output rates of the trigger stages are shown.

With a bunch crossing frequency of roughly $40 \mathrm{MHz}$ and an average number of more than 20 interactions per bunch crossing, the total event rate at the ATLAS interaction region is larger than $1 \mathrm{GHz}$. This rate has to be reduced to a rate at the order of some $100 \mathrm{~Hz}$, which at the ATLAS experiment is achieved by a three-level trigger system, as shown in Figure 3.8.

The first level of the trigger system is called L1. This trigger is based on hardware only in order to ensure a fast trigger decision at this stage. It is designed to accept or reject an event 
with a maximum latency of $2.5 \mu \mathrm{s}$. This time is longer than the design $25 \mathrm{~ns}$ bunch spacing, such that pipeline memories are used to store the data until the trigger decision for an event has been taken by the central trigger processor (CTP). The CTP processes an input of up to 256 different trigger bits. This input can come from the different subsystems as described above and is used to select events with either of the following objects: Muons, electromagnetic clusters (electrons, photons), narrow jets ( $\tau s$ and isolated hadrons), and jets. Depending on the trigger signature, these objects are required to exceed certain energy and/or transverse momentum thresholds based on look-up tables. Furthermore the L1 trigger can select events with certain global properties, such as the missing transverse energy and the total scalar transverse energy. For most objects, information from the calorimeters is used, which at L1 is divided into towers of size $0.1 \times 0.1$ in terms of $\delta \eta \times \delta \phi$. For triggering on muons, hit information from the muon trigger chambers are used - this will be detailed later in this section.

If an event is accepted by the L1 trigger, a small fraction of the total event data can be accessed by the next trigger level. To define the data that is to be analysed, the L1 trigger creates so-called regions of interest (RoI), for localised objects typically defined by their position and their transverse momentum. The RoIs indicate the regions and hereby the fraction of data that needs to be analysed by the second trigger level.

The second level of the trigger system is referred to as L2. It uses data around the RoIs it gets from L1 to confirm and refine the L1 trigger decision. In a first step the object identification of the L1 trigger is confirmed by using more fine-grained information from the same detector systems that the L1 trigger decision was based on. In a second step, additional information from other detector parts are used to refine the trigger decision, for instance tracking information from the ID to identify high $\mathrm{p}_{T}$ leptons. At L2 the trigger is designed for a maximum latency of about $10 \mathrm{~ms}$ per event. If an event passes L2, it is passed on to the third level of the trigger, the Event Filter (EF).

The EF can access the full event data with the full granularity. With a processing time at the order of some seconds, it is possible to use reconstruction algorithms similar to the ones that are used in the offline reconstruction, although there are certain differences. Usually the EF is instructed by L2 which objects in which regions it can use to select or reject an event, but for combined triggers there are more options, as explained below.

At each level a selection of trigger items is defined; for instance the item L1_EM10 defines an item at L1 requiring an electromagnetic object passing the hypothesis EM10. A hypothesis for L1, L2 and EF objects is usually a selection of cuts to which these objects are subject. If the object passes all cuts, it passes the hypothesis, and depending on the number of objects passing a certain hypothesis the event is passed to the next trigger stage (L1 and L2)/registered for permanent storage $(\mathrm{EF})$, or rejected. By construction, an event has to pass each trigger level in order to be selected for physics analyses. Related to this feature, triggers on certain physics objects are combined to trigger chains, such that for instance an L2 item is seeded by an L1 item triggering on the same type of object, as well as an EF item is seeded by an L2 item triggering on the same type of object. It is also possible to add a requirement at one of the higher levels; for instance, an EF item selecting events with at least two muon candidates can be seeded by an L2 item which requires only one muon; these are called full scan triggers (EFFS).

It is also possible to run a trigger item in pass-through mode, which means that an event is passed to the next stage without taking into account if the trigger object passes the hypothesis. With increasing instantaneous luminosities, the trigger thresholds must be increased in order to maintain a feasible rate of events passing the EF. A trigger item that would select events at a rate that is too high by a factor of $n$ can then be prescaled, i.e. only every $n^{\text {th }}$ event passing the trigger hypothesis is accepted. The prescale can be applied at any of the trigger levels, but it 
is usually necessary to prescale L1 or L2 items. If an event is rejected by some trigger chain $A$ due to a prescale at L2, the trigger hypothesis for the corresponding EF item is not necessarily checked online. If however the same event is accepted by a different trigger chain $B$, the EF hypothesis of the trigger chain $A$ can be obtained by re-running the trigger offline.

The Muon Trigger in ATLAS As it is of relevance for parts of this thesis, the ATLAS muon trigger system is explained in a bit more detail.

At L1 the muon trigger uses hit information from the RPCs in the barrel and the TGCs in the endcaps. The procedure is explained by means of Figure 3.9. In a first step a pivot plane is defined, which in the barrel is the plane of muon trigger chambers nearest to the interaction point, and in the endcaps is the plane of muon trigger chambers farthest from the interaction point. For triggering on low $p_{T}$ muons, each hit in an RPC or a TGC in the pivot plane is extrapolated to the second layer of trigger chambers along a straight line through the interaction point. A coincidence window with a certain size (depending on the $p_{T}$ threshold) is defined around the intersection point, and if a hit is found within such a coincidence window the trigger hypothesis is passed. A similar procedure is used to trigger on high $p_{T}$ muons with the option to use all three layers of muon chambers.

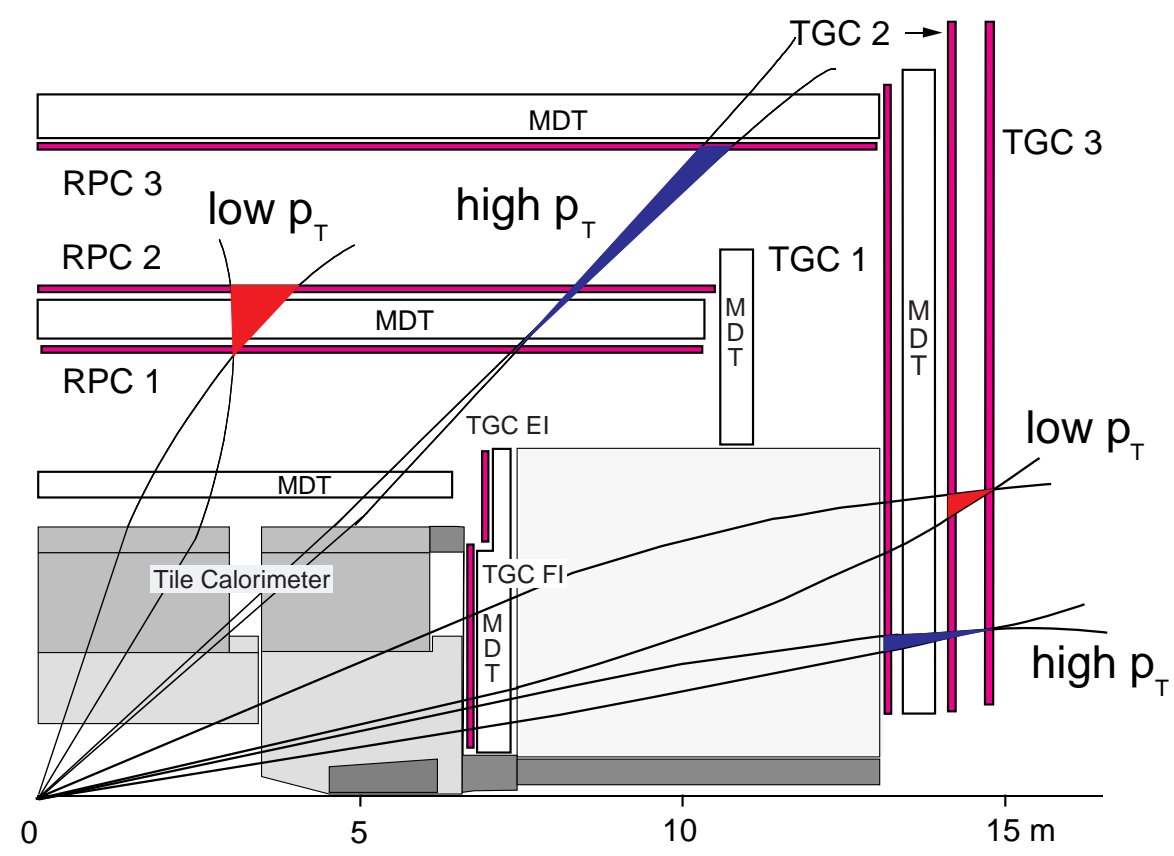

Figure 3.9: The ATLAS L1 muon trigger

At L2 fast tracking algorithms are used to reconstruct muon candidates. It is possible to include the ID tracking information for this to form so-called combined muon candidates. If the track passes the cuts as defined in the trigger hypothesis, the event is passed on to the EF. At the EF, reconstruction algorithms close to those used in the offline reconstruction are used, but with possibly different alignment constants. This allows a larger variety of cuts and quality criteria on muons at the EF.

Trigger Streams Usually the events registered for physics and performance analyses are assigned to so-called trigger streams. A stream is defined by a list of triggers, and an event is 
assigned to a stream if it passes at least one of the triggers in that list. It is possible that an event is assigned to two or more streams at the same time (inclusive streaming). Usually triggers selecting events with similar signatures are collected in a stream. The most relevant streams for physics analyses with the data taken by the ATLAS detector are a stream assembling nearly all trigger chains including a muon trigger item (physics_Muons), a stream assembling nearly all trigger chains including an electron or photon trigger item (physics_Egamma) and a stream with trigger chains containing at least one trigger item selecting events with high $p_{T}$ jets, $\tau \mathrm{s}$ or a large amount of missing transverse energy (physics_JetTauEtmiss).

\subsubsection{Performance of the ATLAS detector}

Of particular importance for searches for new physics is the comparison of the expected performance with the actual performance of the detector, as usually Monte Carlo simulation (MC) is used to estimate the shapes and normalisation of the new physics contributions to distributions of variables that are sensitive to the new physics models. There is a large number of measurements that are performed to explore how well the different subsystems of the ATLAS detector are performing, such that only a small overview of some measurements is given here. While certain parts of the detector simulation can be replaced by reweighting techniques, cf. chapter 5 , for other features of the simulation this is not feasible, and discrepancies between the detector performance in simulation and during data-taking must be corrected for. As the quantities that are to be compared between data and MC usually vary as a function of the detector coordinates - in particular $\eta$ and $\phi$ - and as the angular distributions of particles that can be used to measure these quantities depend on the hard process they originate from, one or more physics processes are usually selected and a background subtraction is performed on data. The determination of correction factors is then performed on the dataset corresponding to that physics process only.

For instance, the determination of the energy-scale for electrons can be done by comparing the electron energy $E$ as measured in the ECAL with the total momentum $p$ of the electron as measured in the ID. Figure 3.10 shows the distribution of the quantity $E / p$ for electrons and positrons that originate from the decay $W \rightarrow e \nu$. The most probable value $\hat{E / p}$ is determined using data and MC, and a correction factor $\alpha_{E / p}$ is calculated using $\hat{E / p_{\text {data }}}=\hat{E / p_{\mathrm{MC}}}\left(1+\alpha_{E / p}\right)$. A detailed description of the measurement, and measurements using different physics processes, can be found in [82].

Another important property of the detector is the ability to identify heavy flavour jets, mainly $b$-jets. The procedure is referred to as $b$-tagging and can be used for a systematic selection or rejection of events containing one or more heavy flavour jets. There are several algorithms that can be employed for $b$-tagging, see for instance [84, 85]. Figure 3.11 shows the $b$-tagging efficiency using a neural network at a working point with a design efficiency of $70 \%$ as a function of the $b$-jet transverse momentum. The measurement was done with a method that uses $b$-jets containig a muon, exploiting the fact that for $b$-jets the muon momentum transverse to the combined jet-muon axis tends to be larger than for light flavour jets containing a muon. Aside from the absolute efficiency as measured from data, a comparison between that efficiency and the efficiency measured in a MC sample is shown [86].

As another example Figure 3.12 shows the resolution for the di-muon mass using $Z \rightarrow \mu \mu$ decays with data from 2012 [87]. The resolution is shown as a function of the muon $\eta$ and is compared between data and $\mathrm{MC}$ before and after a correction factor has been applied to the MC. Also, the figure shows the reconstruction efficiency for combined muons (ID+MS) for two different sets of hit-criteria in the muon spectrometer. 


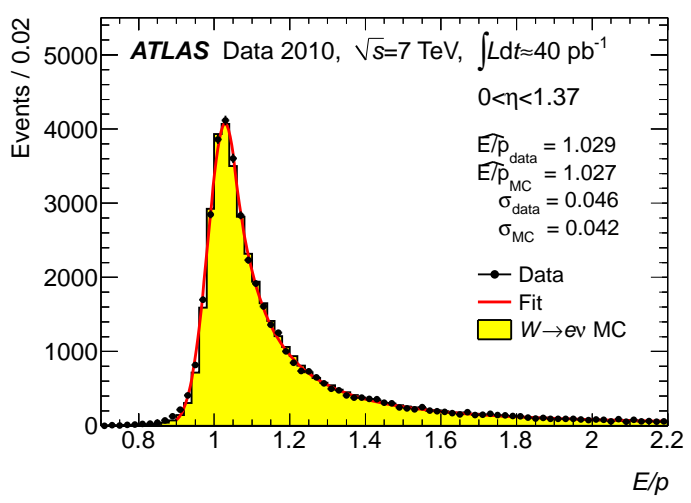

(a)

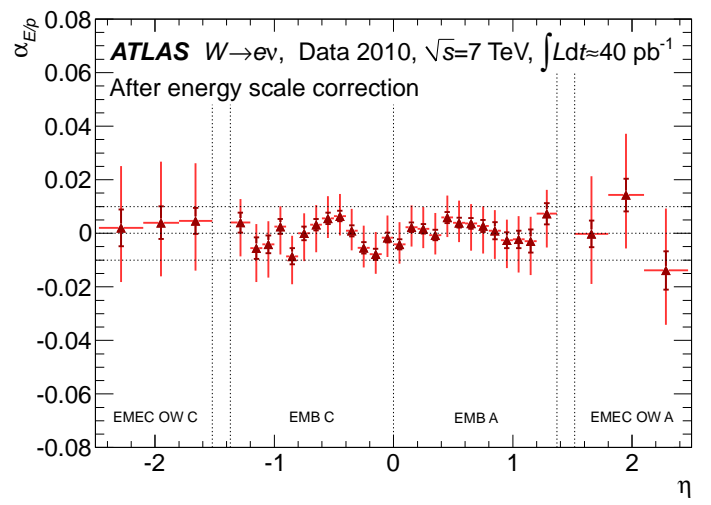

(b)

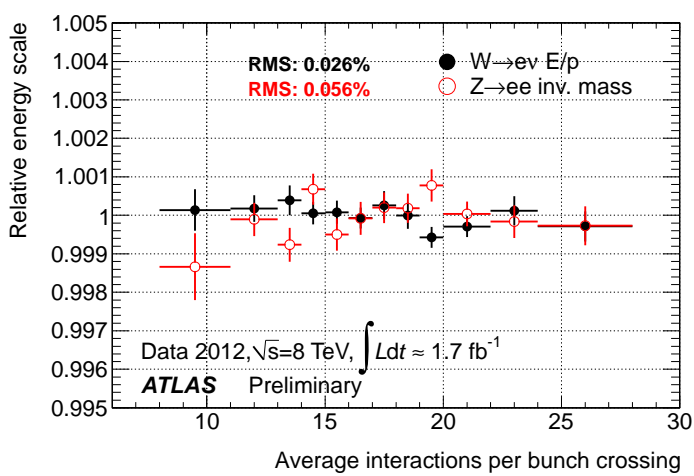

(c)

Figure 3.10: Top: The plot on the left hand side shows the distribution of $E / p$ for electrons and positrons as measured in $W \rightarrow e \nu$. The distribution inlcudes all electrons with $0<\eta<1.37$. The plot on the right hand side shows the correction factors for the electromagnetic energy scale as a function of $\eta$ for electrons from $W \rightarrow e \nu$. Both figures are taken from [82]. Bottom: This plot shows the stability of the energy-scale as a function of the average number of interactions $\langle\mu\rangle$, determined by measurements with a dataset of $1.7 \mathrm{fb}^{-1}$ in 2012 using different techniques, where the centre of mass energy for the p-p collisions was increased to $8 \mathrm{TeV}$ [83]. 


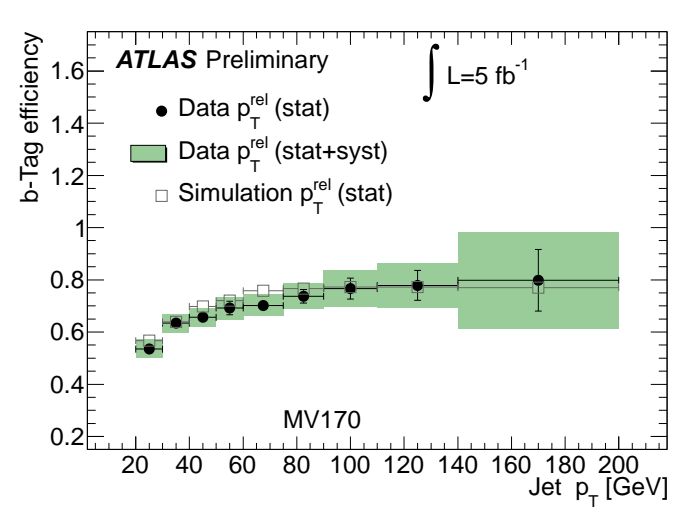

(a)

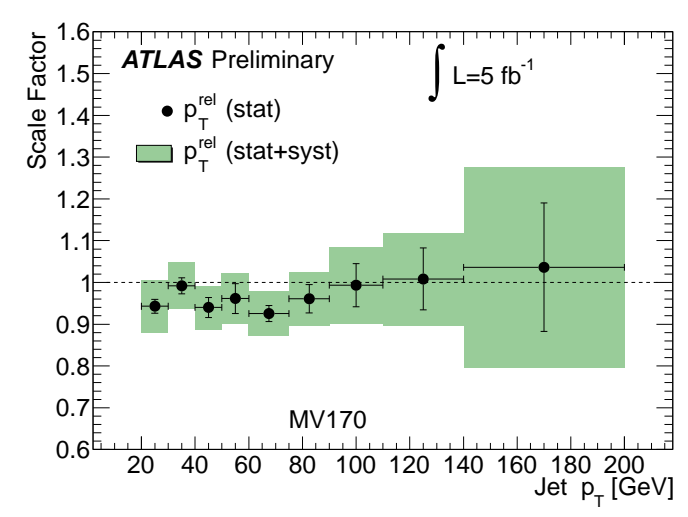

(b)

Figure 3.11: The plot on the left hand side shows the measured $b$-tagging efficiency as a function of the $b$-jet transverse momentum with the so-called $p_{T}^{\mathrm{rel}}$-method [86]. On the right hand side, a comparison between MC and data is shown.

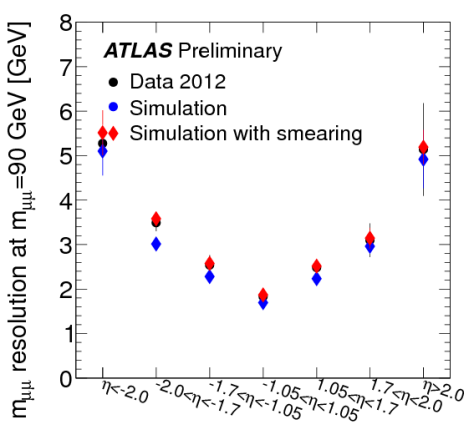

(a)

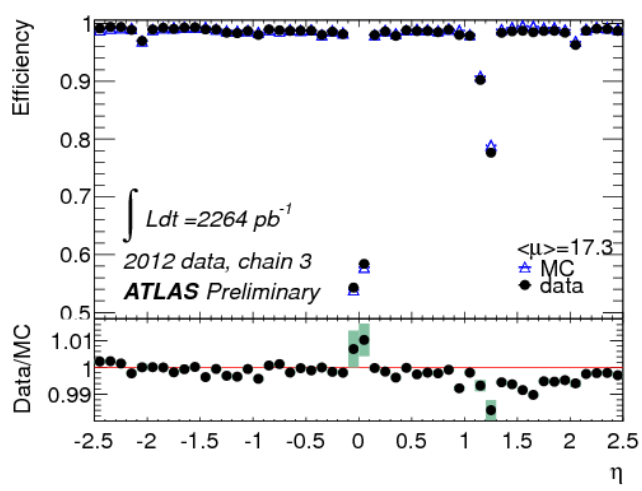

(d)

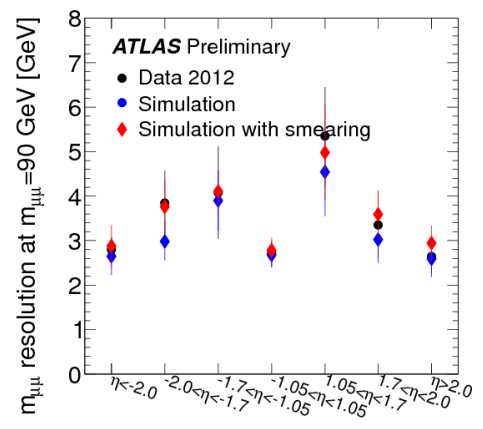

(b)

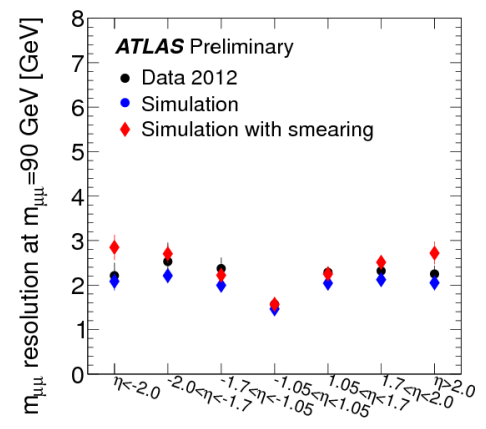

(c)

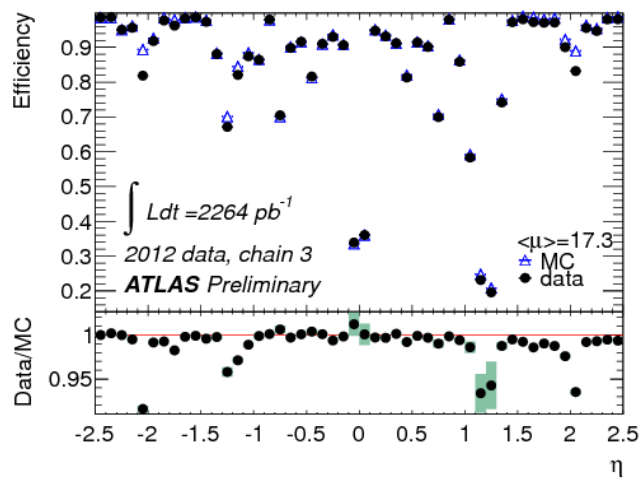

(e)

Figure 3.12: Top: The di-muon mass resolution from $Z \rightarrow \mu \mu$ decays using (a) the inner detector only, (b) the muon spectrometer only and (c) a combined measurement. The plots show a comparison between $\mathrm{MC}$ and data before and after a smearing correction was applied to the MC. Bottom: The reconstruction efficiency for combined muons, with requiring (d) two hits and (e) three hits in the muon chambers [87]. 


\section{The CMSSM and NUHM1 After Two Years of LHC Operation}

If the SM of particle physics is extended to include so-called new physics, the predictions for a variety of observables change compared to the SM predictions. A number of observables which possibly provide sensitivity to new physics models is available, and the predictions that these new physics models make can be compared against the measured values, inferring constraints on the parameters that enter the Lagrangian density of the extended SM. Apart from observables at the $\mathrm{GeV}$ scale, direct searches for physics beyond the SM (BSM) are performed, and the exclusion limits set by the experiments at the LHC can be employed to further constrain the BSM models.

Since it is in general not possible to calculate the model parameters directly from the observables, these parameters are usually constrained by global fits; the parameter space of a BSM model is sampled and at each point in the parameter space the predictions are compared to the observations; statistical methods are then used to derive boundaries on the parameters. Here, theoretically already highly constrained SUSY models are subject to such a fit.

\subsection{Global Fits of SUSY Models}

The results of global fits of the CMSSM and the NUHM1 to a set of measurements are presented. After a brief introduction of the general idea, the Fittino [88, 89, 90, 91, 92, 93] framework is described and the available measurements and their relevance to such fits are summarised. An analysis that has been performed using measurements available in 2011 and 2012 is presented with a focus on fine-tuning, which has been studied in the Fittino collaboration for the first time, here. The results presented in this thesis are a part of a more complete study of the CMSSM and the non-universal Higgs mass models, the most important results of which will be briefly summarised here. For a more detailed discussion of the employed methods, the used observables and the results, see the original paper [94].

\subsubsection{The Idea}

As detailed in chapter 2, even the minimal supersymmetric extension of the SM introduces a huge number of new parameters in the Lagrangian density. By establishing constraints on some of these, the number of independent parameters can be significantly decreased, as it is done for instance in the CMSSM. However, no matter what the number of new parameters is, there is no way to predict their actual values from theory considerations, only. Measurements are needed in order to determine the values of the parameters and so be able to make testable predictions for the model in question.

In the pMSSM, as well as in most of the more constrained models, nearly all parameters describe the masses or the couplings of the supersymmetric particles. The lack of any direct observations of such particles so far naturally aggravates the determination of the parameters of the underlying Lagrangian density. While the missing observation of any direct signal can be 
used to set usually one-sided boundaries on a subset of parameters, the part of the parameter space that can not be excluded by past and recent direct searches is huge, and the limits that are set usually apply only under certain conditions.

Even if at some point one or more of the superpartners of the SM particles are found and their masses and couplings are measured, the determination of the parameters of a supersymmetric Lagrangian density is of high interest in particular for a better understanding of the fundamental SUSY breaking mechanism. This task is not straight forward, because there is no one-to-one correspondence between observables and the parameters in the Lagrangian density; for instance, a mass parameter in the Lagrangian density does usually not acquire the same value as the corresponding observable mass. One reason for this is that the parameters as they are defined in the MSSM Lagrangian are usually attributed to the gauge eigenstates, which due to mixing may significantly differ from the physical mass eigenstates; this is in particular true for the superpartners of the electroweak gauge bosons and Higgs bosons, but also for squarks and sleptons. Furthermore, loop diagrams contribute to the masses and couplings of the particles; for instance, the more constrained models with only a few parameters are usually defined at some very high energy scale (GUT scale), that lies orders of magnitude above the scales that are probed by experiments. The parameters have to be calculated at the experimental scales using the renormalisation group equation in such a case, to predict the physical masses and couplings at the appropriate energy scales. Depending on the number of loops that are taken into account, the MSSM $\beta$-function for any parameter depends on an arbitrarily large subset of all other parameters.

It is on the other hand by these loop corrections that boundaries on the SUSY breaking parameters can be set that are tighter than the bounds that are set due to the non-observation of any superpartners in direct searches. In particular measurements in the sector of flavour physics are of interest here; for instance, rare decays of some $B$ - and $K$-mesons can significantly be enhanced by supersymmetric loop contributions, where in the Feynman diagrams the superpartners occur only as internal lines.

For a fixed set of SUSY breaking parameters it is possible to calculate predictions for all relevant observables - the reverse is usually not possible, as the $\beta$-functions can introduce highly non-linear, non-invertable relations between parameters and observables. The preferred technique is therefore to sample the parameter space of a well defined model and compare the predictions for each set of parameter values - or point - to the measurements. Statistical methods can then be used to evaluate the agreement between predictions and observations, and derive boundaries on model parameters, or even completely exclude a model. For a meaningful result of a scan it is mandatory to perform the sampling of the parameter space in a smart way - a simple scan with aequidistant grid points in each dimension is neither sufficient nor efficient, even for a relatively small number of dimensions.

\subsubsection{The Fittino Framework}

The Fittino framework can be used to perform scans of the parameter space of several models. Following a top-down approach the parameters at a point are handed to external theory codes that are used to calculate predictions for all relevant observables; the predictions are reported back to Fittino and compared to the available measurements by calculating a $\chi^{2}$. Different scanning algorithms can be used to identify the point in parameter space describing the relevant observations best and study the characteristics of the model around that minimum.

In this section the theory codes that are used by Fittino are briefly described. Afterwards the scanning algorithm that has been used for all results shown in this chapter is specified and 
the statistical interpretation of the scan results is detailed.

\section{Theory Predictions}

In a high-scale model like the CMSSM or the NUHM1, the first step in the prediction of observables at the electroweak symmetry breaking scale $Q_{\mathrm{EWSB}}$ is the calculation of the soft SUSY breaking parameters and the calculation of the masses and couplings of the physical states of the superpartners at that scale. There is a variety of codes that perform these calculations, which will be referred to as spectrum calculators in the following. For the results presented in this thesis, the spectrum calculators SPheno [95], version 3.1.4, and SoftSUSY [96], version 3.1.7, have been used. The codes have been found to yield comparable results for the most relevant regions of the parameter space, although the differences can get large for large values of the mass parameters. For the study presented here, the electroweak symmetry breaking scale has been fixed to $Q_{\mathrm{EWSB}}=1 \mathrm{TeV}$ in accordance with the SPA conventions [97].

The spectrum is passed to micrOMEGAs $[98,99]$, version 2.2, which calculates the cold dark matter relic density, and to FeynHiggs [100], version 2.8.2, from which the predictions of the Higgs masses, the mass of the $W$ boson, the effective weak mixing angle $\sin ^{2} \theta$ and the anomalous magnetic moment of the muon $a_{\mu}$ are obtained. The program SuperISO [101], version 3.1 is used to calculate the predicted values for the flavour observables. In addition to the relic density $\Omega_{\mathrm{CDM}}$ the results of direct and indirect dark matter detection experiments can be included in the constraints via AstroFit [102]. Finally, the prediction of the branching fractions of the lightest Higgs boson is performed by HDECAY [103] version 4.41 .

\section{Parameter Sampling and Calculation of the $\chi^{2}$}

The parameter space is scanned using a Markov Chain Monte Carlo (MCMC), the progression of which is defined by a single measure for the agreement between data and predictions for all used observables. This measure is the $\chi^{2}$, which is calculated in three steps. For all available measurements the $\chi^{2}$ contribution is defined as

$$
\chi^{2}=\sum_{i=1}^{N_{\text {obs }}}\left(\frac{O_{\text {meas }}^{i}-O_{\text {pred }}^{i}(\vec{P})}{\sigma_{O^{i}}^{2}}\right)^{2} .
$$

In a second step, upper bounds contribute to the $\chi^{2}$ only if the prediction lies above that bound,

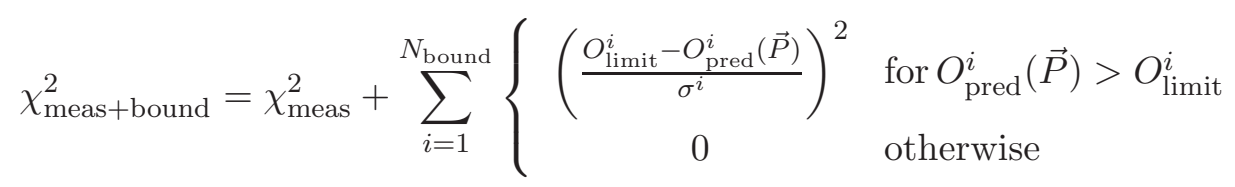

Finally the $\chi^{2}$ contributions from AstroFit and the LHC limit are added. The calculation of these is detailed later.

In the MCMC, the $\chi^{2}$ is used to calculate a likelihood $L=e^{-\frac{\chi^{2}}{2}}$ for a given point. After the calculation of $L_{n}$ at one point $P_{n}$, the fitted model parameters are varied according to proposal distributions around that point, defining a new point $P_{n+1}$. If $L_{n+1} / L_{n} \geq 1$, the new point is added to the Markov Chain. If $L_{n+1} / L_{n}=p<1$, the new point is added to the Markov Chain with a probability $p$; if it is rejected, the point $P_{n}$ is again added to the Markov Chain and a new point $P_{n+1}^{\prime}$ is tested. This procedure ensures that the scan tends to converge towards the global minimum, while it is is still able to escape from local minima in the parameter space. More details on the algorithm can be found in [91]. 
The MCMC algorithm employed for the studies presented in this chapter makes use of a continuous optimisation of the proposal widths for all parameters, which is based on the variance of the parameters at the accepted points. Different ratios of that variance and the proposal widths have been used in order to allow for a fine scan near the minimum, as well as a reasonable coverage of the complete parameter space. The Markov Chains that have been used for the study that is presented here have a length of several million points, typically. More details can be found in [94].

\section{Statistical Interpretation of Scan Results}

Two fundamentally different approaches to statistical interpretation exist. In the Bayesian interpretation, the point density in the MCMC is regarded as a probability density function for the parameters, from which intervals corresponding to a certain confidence level can directly be calculated. In the Frequentist interpretation the intervals are calculated based on the probability to get a result as bad or worse than the actual result, provided that the tested model, or point in the parameter space, is realised. For this study, the Frequentist interpretation was chosen: First, the point with the smallest $\chi^{2}$ is identified - for all fits, this point is referred to as the minimum or the best fit point in the following. Starting from the minimum with an agreement of $\chi_{\min }^{2}$ between data and predictions, in the projections of the results on one (two) parameter (s), the one-dimensional $1 \sigma$ (2-dimensional $2 \sigma$ ) boundaries are defined by $\Delta \chi^{2}=\chi^{2}-\chi_{\min }^{2}<1(5.99)$, where the profiling technique is used for the hidden dimensions, i.e. the hidden dimensions are scanned and only the point with the smallest $\chi^{2}$ is considered. Note that an exact overlap of the $1 \sigma(2 \sigma)$ contours with the $68 \%$-CL $(95 \%$-CL) intervals can not be claimed; also, the calculation of a reliable p-value for the best fit point can only be done using toys, because the underlying distribution for the $\chi^{2}$ is not necessarily given by the gaussian $\chi^{2}$-distribution for the respective number of degrees of freedom. This topic will be picked up in one of the follow-up analyses performed by the Fittino collaboration [104].

As outlined above, the MCMC can be interpreted in a Bayesian way as well; for several reasons this is not the preferred interpretation for the study presented here. Since the available measurements constrain the parameter space only weakly, there is a significant dependence of the fit results on the chosen prior p.d.f.. Apart from the difficulty to define a reasonable prior distribution for the model parameters, the dynamic adjustment of the proposal widths in the Fittino implementation of the MCMC will introduce a bias in the interpretation of the MCMC local density as a posteriori p.d.f.. To illustrate the strong dependence of the fit results on the choice of the prior distributions, a comparison of the Frequentist and Bayesian interpretation with different priors has been performed in the $M_{0}-M_{1 / 2}$ plane, using an example scan of the CMSSM parameter space with a negligible variation in the proposal widths for all parameters.

The $m$-dimensional marginalised posterior pdf for $m$ parameters is drawn from the full $n$ dimensional posterior pdf by integrating out all other parameters,

$$
p_{\text {marg }}^{m}\left(\vec{P}_{i}\right)=\int p_{\text {full }}^{n}\left(\vec{P}_{i}, \vec{P}_{j}\right) d^{n-m} P_{j}
$$

where $p_{\text {full }}^{n}\left(\vec{P}_{i}, \vec{P}_{j}\right)$ is given by the MCMC point density and $j(i)$ runs over all parameters that are (not) removed by integration. In the case of a non-flat prior p.d.f., this is multiplied to $p_{\text {full }}^{n}$ at each point. The corresponding $1 \sigma(2 \sigma)$ boundaries are defined by the smallest interval covering $68 \%$ (95\%). The interval is built iteratively from the binned, marginalised posterior pdf by ordering all bins according to their probability $p_{i}$, starting with the highest probability. 


\begin{tabular}{l|r|c|c}
\hline \hline parameter & best fit (freq.) & global mode (Bayesian) & local mode (Bayesian) \\
\hline $\tan \beta$ & $10.3_{-4.7}^{+9.5}$ & 8.2 & $3.5_{-1.3}^{+21.3}$ \\
$M_{1 / 2}[\mathrm{GeV}]$ & $288.1_{-58.3}^{+99.0}$ & 270.3 & $143.5_{-30.5}^{+377.5}$ \\
$M_{0}[\mathrm{GeV}]$ & $58.3_{-14.9}^{+87.0}$ & 52.4 & $58.5_{-42.5}^{+505.5}$ \\
$A_{0}[\mathrm{GeV}]$ & $259.8_{-570.1}^{+686.9}$ & 23.5 & $403.0_{-2027.0}^{+2379.0}$ \\
\hline \hline
\end{tabular}

Table 4.1: Results of an example fit for the Frequentist and the Bayesian interpretation as published in [94]. For the Frequentist interpretation the point with the smallest $\chi^{2}$ is given with the corresponding 1-dimensional $1 \sigma$ uncertainties. For the Bayesian interpretation, the global mode of the full posterior pdf in the case of flat priors is given, i.e. the point with the highest local $4 \mathrm{D}$ point density. In addition the maxima of the marginalised 1-dimensional posteriors are shown with the boundaries of the smallest interval covering $68 \%$ around the maximum.

Then the $p_{i}$ are subsequently added, until $68 \%(95 \%)$ is reached:

$$
\sum_{i=1}^{\max _{\mathrm{nbin}}} p_{i}<0.68(0.95) \text {. }
$$

The allowed region is the one which contains all bins in the range $\left[1, \max _{\text {nbin }}\right]$. The remaining bins are outside of the allowed region. In this way, both the 2-dimensional $2 \sigma$ areas as well as the 1-dimensional (local) modes and the corresponding $1 \sigma$ intervals are constructed from the full posterior pdf.

A comparison of both interpretations is shown in Table 4.1 and Figure 4.1. In Figure 4.1, the black lines indicate the 2 -dimensional $2 \sigma$ regions calculated using the Frequentist interpretation (a), or the Bayesian interpretation with a different choice of prior distributions, (b) to (d). It is important to note that in the Frequentist interpretation also points that are rejected by the Markov Chain are eventually considered, which is not the case in the Bayesian interpretation.

To conclude, the Frequentist interpretation allows for a variation of the MCMC proposal widths during the run of the scan. For this reason, and for the lack of any well motivated choice for a prior distribution for the model parameters - in combination with the significant dependence of the final result on the choice of the prior - a Frequentist interpretation of the MCMC seems more sensible in this case.

\subsubsection{Observables}

There are different types of observables that have been used for the study presented in this thesis. The first set consists of measurements from high energy physics that have been performed at experiments before the era of the LHC. These include measurements from the $B$-factory experiments BaBar and Belle and the measurements performed at LEP. These observables are referred to as Low Energy Observables (LEO), although for some of these measurements there have been updates from the LHC experiments already. The second set of observables are the exclusion limits set by the direct searches at the LHC experiments, from which usually only the limit from the analysis with the highest expected sensitivity is used. Finally, there are cosmological observations that can as well be used for constraining supersymmetric models. 


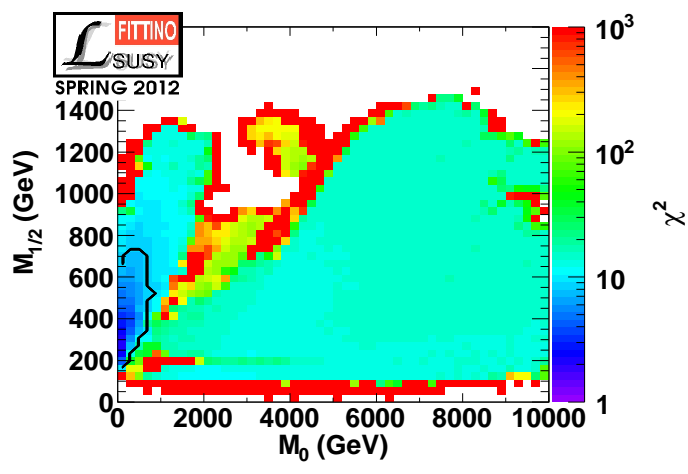

(a)

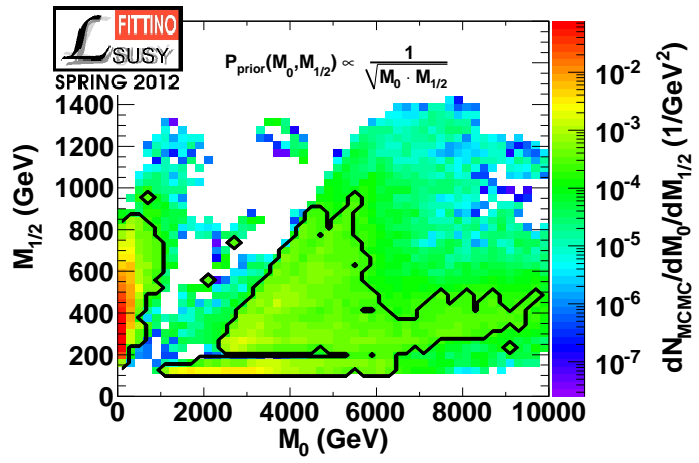

(c)

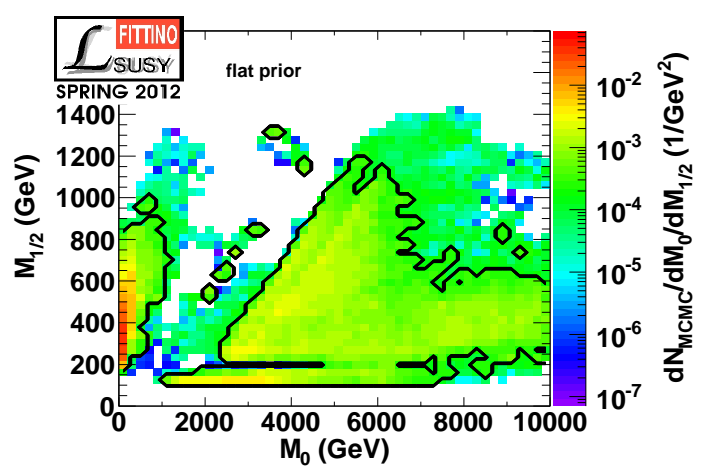

(b)

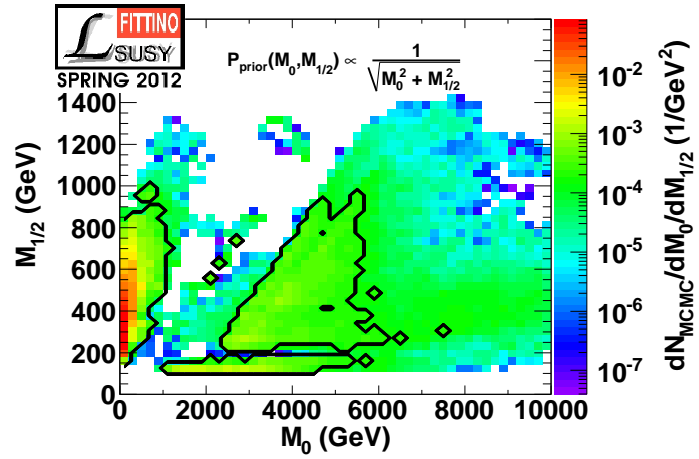

(d)

Figure 4.1: Comparison of different statistical interpretations of the same example fit based on [105]. (a) Frequentist profile likelihood; (b) Bayesian marginalisation with flat prior; (c) and (d): two different non-flat priors, as described in the Text. The black lines indicate the $2 \sigma$ (Frequentist) and 95\% CL (Bayesian) contours, respectively [94].

Before the relevant observables from each category are briefly described, note that some of the parameters that appear in a SUSY Lagrangian are already known; since the MSSM is an extension to the SM, it is not only the soft SUSY breaking parameters that occur in the Lagrangian, but also some of the SM parameters. As an example, for a high scale model, the SM gauge couplings are needed to determine the unification scale, at which the model is defined. These parameters have been fixed at the values given in Table 4.2, with the exception of the top mass, which has been allowed to vary according to its uncertainty in some of the fits, as detailed later in this chapter.

\section{Low Energy Observables}

The area of LEOs can be further subdivided into three major types of observables.

First there are the electroweak precision observables from LEP and the SLC. Most of these observables, like the width, the mass and the couplings of the $Z$ boson have been found to have a negligible impact on the fit and are therefore not taken into account here. The observables with the highest relevance are the effective electroweak mixing angle, $\sin ^{2} \theta_{W}$, and the mass of the $W$ boson. 


\begin{tabular}{l|c|c}
\hline \hline $1 / \alpha_{\mathrm{em}}$ & 128.962 & {$[106]$} \\
$G_{\mathrm{F}}$ & $1.16637 \times 10^{-5}$ & {$[107]$} \\
$\alpha_{\mathrm{S}}$ & 0.1176 & {$[107]$} \\
$m_{Z}$ & $91.1876 \mathrm{GeV}$ & {$[107]$} \\
$m_{b}$ & $4.19 \mathrm{GeV}$ & {$[107]$} \\
$m_{t}$ & $(173.2 \pm 1.34) \mathrm{GeV}$ & {$[107]$} \\
$m_{\tau}$ & $1.77682 \mathrm{GeV}$ & {$[107]$} \\
$m_{c}$ & $1.27 \mathrm{GeV}$ & {$[107]$} \\
\hline
\end{tabular}

Table 4.2: Standard Model parameters that have been fixed, apart from $m_{t}$ in one particular fit [94].

Second, there is the anomalous magnetic moment of the muon, $a_{\mu}=\frac{1}{2}(g-2)_{\mu}$. The accuracy of the SM prediction of this observable is not unproblematic, because the non-perturbative hadronic contribution to $a_{\mu}$ has to be determined from experiments. The value that has been used for the study presented here has been calculated using data from $e^{+} e^{-}$annihilation to hadrons. Compared to the anomalous magnetic moment of the electron, which has been measured with a much better precision, the sensitivity of $a_{\mu}$ to new physics is significantly enhanced due to the large mass of the muon.

The third large group of LEOs are rare decays of $B$ mesons. In particular processes that are prohibited at tree level, but allowed if loop diagrams are taken into account, provide strong constraints on physics beyond the SM. Most important of these observables are the inclusive branching fraction of $b \rightarrow s \gamma$, the branching fraction of $B \rightarrow \tau \nu$ and the branching fraction of $B_{s} \rightarrow \mu \mu$. At the time this particular study has been performed, only an upper limit was available for the latter; in the meantime the LHCb collaboration has published the observation of the decay $B_{s} \rightarrow \mu \mu$ with a branching fraction that is agreement with the SM prediction [108]. The implications of that are also studied in [94], for instance. New physics can as well be constrained by taking into account the oscillations of $B_{s}$ mesons.

All low energy observables that have been employed for the study presented here are summarised in Table 4.3. While there is a large number of additional LEOs that may be sensitive to physics beyond the SM, these do not have a significant impact on fits of the CMSSM and NUHM1 and are therefore not taken into account, here (for a more complete discussion of this issue, see [94]).

\section{Limits on Particle Masses from LEP}

The experiments at LEP have performed searches for charginos and set limits on the chargino mass under the assumption of gaugino mass parameter unification at the GUT scale. Since this assumption holds for the CMSSM and the NUHM1, the limits have been used in this analysis. A lower bound of $m_{\tilde{\chi}_{1}^{ \pm}}>102.5 \mathrm{GeV}$ has been used as a constraint here, considering a theoretical uncertainty of $1 \mathrm{GeV}$. Due to the unification of the gaugino mass parameters, the limit on the chargino mass translates into a limit on the mass of the lightest neutralino, such that $m_{\tilde{\chi}_{1}^{0}}>50$ $\mathrm{GeV}$ is required $[107,117,118,119]$.

The collaborations at LEP and the LHC have also carried out searches for the Higgs boson. At the time this study was performed, the data taken at the LHC had shown strong hints to the existence of a Higgs boson with a mass of about $126 \mathrm{GeV}$, although evidence could not yet be claimed $[120,121]$. For the various results that are discussed in the next section, two different 


\begin{tabular}{l|c|c}
\hline \hline $\mathcal{B}(b \rightarrow s \gamma)$ & $(3.55 \pm 0.34) \times 10^{-4}$ & {$[109]$} \\
$\mathcal{B}\left(B_{s} \rightarrow \mu \mu\right)$ & $<4.5 \times 10^{-9}$ & {$[110]$} \\
$\mathcal{B}(B \rightarrow \tau \nu)$ & $(1.67 \pm 0.39) \times 10^{-4}$ & {$[109]$} \\
$\Delta m_{B_{s}}$ & $17.78 \pm 5.2 \mathrm{ps}^{-1}$ & {$[109]$} \\
$a_{\mu}^{\exp }-a_{\mu}^{\mathrm{SM}}$ & $(28.7 \pm 8.2) \times 10^{-10}$ & {$[106,111]$} \\
$m_{W}$ & $(80.385 \pm 0.015) \mathrm{GeV}$ & {$[112,113,114]$} \\
$\sin ^{2} \theta_{\mathrm{eff}}$ & $0.23113 \pm 0.00021$ & {$[115]$} \\
$\Omega_{\mathrm{CDM}} h^{2}$ & $0.1123 \pm 0.0118$ & {$[116]$} \\
$m_{t}$ & $(173.2 \pm 1.34) \mathrm{GeV}$ & {$[107]$} \\
\hline
\end{tabular}

Table 4.3: Low-energy observables employed. In general, experimental and theoretical uncertainties have been added in quadrature. The top quark mass $m_{t}$ is only used as an observable for the fit where $m_{t}$ is also floating as SM input parameter [94].

scenarios have therefore been considered. First, taking into account a theory uncertainty of 3 $\mathrm{GeV}$ for the mass of the Higgs boson, a limit of $m_{h}>114.5 \mathrm{GeV}$ has been used to constrain the CMSSM. Although this limit has been set for the SM Higgs boson, in the CMSSM the lightest Higgs boson is usually very SM like, such that in a good approximation the limit applies to this model as well. In a second generation of fits, the value $m_{h}=126 \pm 3 \mathrm{GeV}$ has been used to constrain both the CMSSM and the NUHM1 models, here.

\section{SUSY Searches at the LHC}

Due to the unification of the mass parameters at a very high scale, the mass differences between the coloured superpartners and the colourless superpartners are usually not too large in both the CMSSM and the NUHM1. Therefore the strongest limits in these models are set by searches at the LHC with jets, missing transverse energy and no leptons in the final state. It is not sufficient to know the 95\%-CL exclusion limits that are set by these searches, but the necessity to calculate a $\chi^{2}$ contribution at any point in the parameter space requires a more detailed consideration of the LHC analyses.

For this Fittino analysis, the search for SUSY performed with the ATLAS detector, using the $\sqrt{s}=7 \mathrm{TeV}$ dataset, in final states with two, three or four jets, missing transverse energy and no leptons has been considered [122]. Amongst all searches, these final states are expected to provide the highest sensitivity to the CMSSM/NUHM1. In order to allow for the proper calculation of a $\chi^{2}$ contribution at any given point in the CMSSM/NUHM1 parameter space, the analysis has been emulated using SPheno 3.1.0 as a spectrum calculator, Herwig 2.4.2 [123] as an event generator, and the fast detector simulation DELPHES [124], version 1.9, where the relevant properties like resolution, efficiency and fake rates of the ATLAS detector have been taken into account considering the public ATLAS measurements. The emulation of the analysis is based on [125] and has been tuned to reproduce the more recent results as published in [122].

For the calculation of the $\chi^{2}$ contribution, the signal event yield is considered as a function of $M_{0}$ and $M_{1 / 2}$. The dependence on $A_{0}$ and $\tan \beta$ has been found to be small in the larger fraction of the relevant parameter space [94]. For very large mass parameters, there is a significant dependence on $A_{0}$ for large absolute values of that parameter - this is studied in much more detail in [104], and the dependence is neglected, here. Following an approach that was first 
presented in [105], the $\chi^{2}$ is calculated from a test statistic $t$, which is defined by

$$
t=-2 \ln \frac{L(s+b, n)}{L(b, n)},
$$

in which $L(\mu, n)$ is the poissonian probability to observe $n$ events where $\mu$ events are expected. Systematic uncertainties on both the background expectation $b$ and the signal expectation $s$ are taken into account by smearing the poissonian distribution accordingly. With the probability density function $P_{s+b}(t)$, assuming the existence of a signal, the $\chi^{2}$ contribution at any point in the $M_{0}-M_{1 / 2}$ plane is given by

$$
\chi^{2}=2 \cdot\left[\operatorname{erf}^{-1}\left(1-2 \mathrm{CL}_{s+b}\right)\right]^{2} \quad \text { with } \mathrm{CL}_{s+b}=\int_{t_{\mathrm{obs}}}^{\infty} P_{s+b}(t) d t .
$$

Here, $t_{\mathrm{obs}}$ is the observed value of the test statistics. Due to the non-observation of any significant excess in the ATLAS search, for the calculation of the $\chi^{2}$ contribution in the Fittino analysis the value $t_{\text {obs }}$ has been calculated by setting $n=b$ in equation (4.5).

A comparison between the Fittino implementation of the ATLAS analysis, and the original analyses carried out by the ATLAS and CMS experiments is shown in Figure 4.2. The agreement between the ATLAS expected limit and the Fittino implementation is reasonable, whereas the differences between the expected limit and the observed limit for the ATLAS analysis is well covered by the uncertainties. More details on the LHC limit implementation can be found in [94].

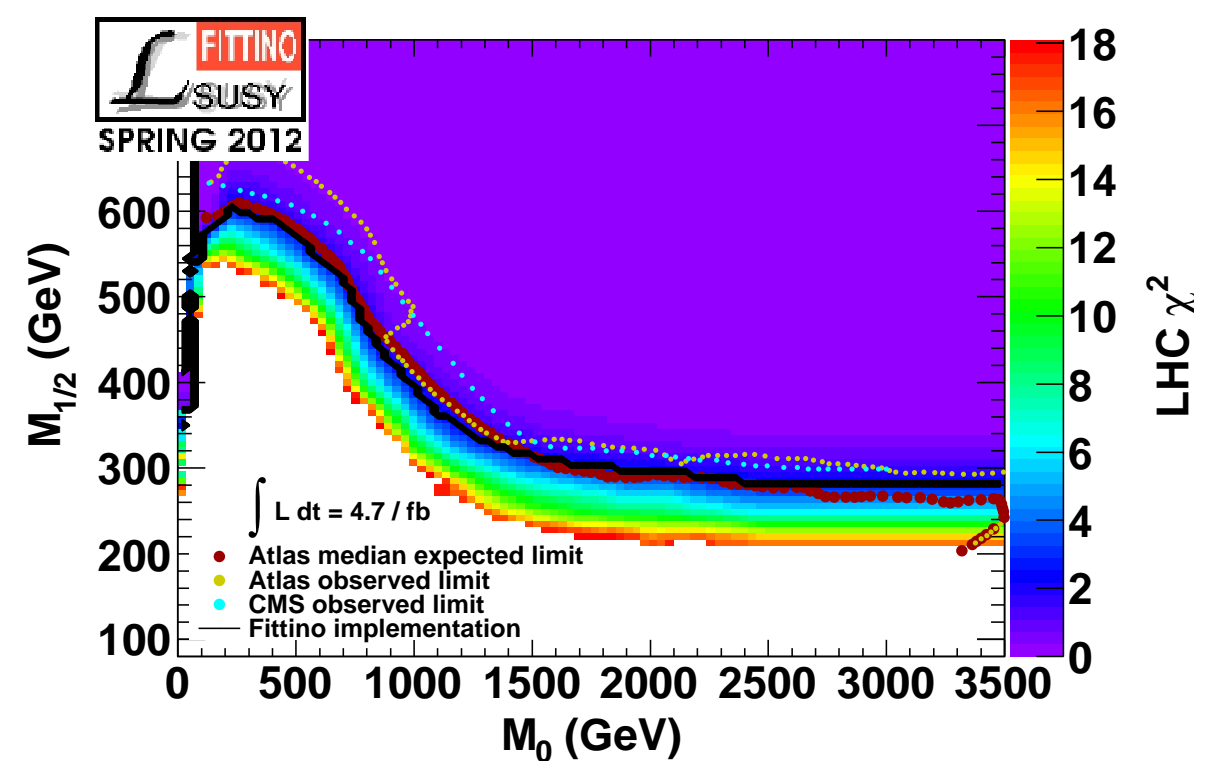

Figure 4.2: $\chi^{2}$ contribution from the LHC SUSY search implementation compared to the published ATLAS and CMS limits $[122,126]$. Good agreement of the estimated limit with the expected limit of the LHC collaborations is achieved [94].

\section{Cosmological Observables}

There are several observables from astroparticle physics that provide potential sensitivity to SUSY, in particular if the LSP is considered to account for a significant part of the dark matter 
in the universe. Most importantly, the relic density of cold dark matter, $\Omega_{\mathrm{CDM}}$, can be used to significantly constrain supersymmetric models. For one of the studies presented in the following chapter, this is the only observable from astroparticle physics that is taken into account.

For the further studies, the Fittino interface to AstroFit has been employed. AstroFit provides an extensive database of several astrophysical measurements and uses the code DarkSUSY [127] to calculate predictions for some observables at a given point in the SUSY parameter space. These observables include the photon, antiproton, electron and positron fluxes as measured by several experiments for indirect dark matter detection $[128,129,130,131,132,133]$, as well as the neutralino-nucleon scattering cross section as it is measured in several direct detection experiments $[54,55,134]$. Using a formula very similar to equation (4.1) for measurements, as well as interpolation and extrapolation techniques for exclusion limits, AstroFit calculates a $\chi^{2}$ contribution for each point in the MCMC and passes it to Fittino. Those results presented in this thesis that rely on AstroFit have been obtained using the dark matter relic density, limits on the dark matter annihilation rates obtained from H.E.S.S. and Fermi-LAT, as well as the limits set on the neutralino-nucleon scattering cross section from Xenon100. More details, in particular concerning the selection of these observables, can be found in [94].

\subsection{The CMSSM/mSUGRA-Model after Two Years of LHC data}

The results of several fits of the CMSSM and the NUHM1, using different sets of observables are presented. After a brief discussion of all results that have been published in [94], a study of the fine-tuning in the CMSSM is presented, where emphasis is put on a comparison of the situations before and after the LHC run at $\sqrt{s}=7 \mathrm{TeV}$. Note that for all fits of the CMSSM and the NUHM1, the sign of the $\mu$ parameter has been fixed to +1 , as this sign is preferred by the observed value of $a_{\mu}$.

\subsubsection{Fit Results}

The results of some of the most important fits that have been performed in [94] are summarised in Table 4.4. The fit name indicates the model that has been fitted and the observable set it has been fitted to. The observable set LEO consists of all observables listed in Table 4.3, augmented by the Higgs exclusion limits set by LEP and the Tevatron experiments (note that for these fits the program SPheno has been used to calculate the SUSY mass spectrum and the program HiggsBounds has been used to calculate a $\chi^{2}$ contribution for the Higgs mass limits). The LHC observable set consists of all LEOs and the exclusion limit from the direct search at the LHC. Finally, the observable set labelled $\mathrm{LHC}+m_{h}$ is given by the observable set LHC, where the lower bound on the Higgs mass is replaced by a measurement of $126 \mathrm{GeV}$ of that quantity.

The 2-dimensional $1 \sigma$ and $2 \sigma$ regions in the $M_{0}-M_{1 / 2}$ plane and in the $A_{0}$-tan $\beta$ plane for fits of the CMSSM to the LEO and the LHC observable set are compared in figures 4.3(a) and 4.3(b). The best fit point is pushed to significantly higher values of $M_{0}$ and $M_{1 / 2}$ by the exclusion limits set by ATLAS. Due to the worse $\chi_{\min }^{2}$ in this fit, regions with relatively large $M_{0}$ and moderate values of $M_{1 / 2}$ are reintroduced in the 2-dimensional $2 \sigma$ region of the LHC fit, as in these regions the LHC limits do not significantly contribute to the global $\chi^{2}$. The best fit value of $\tan \beta$ is also significantly pushed to larger values, although the overlap of the 2 -dimensional $1 \sigma$ regions is large in the $A_{0}-\tan \beta$ plane. The allowed range of $A_{0}$ is significantly enlarged by considering the LHC limits, again at the cost of an overall worse agreement between predictions and observations. 
The preference of larger mass parameters in the LHC fit results in a larger minimum $\chi^{2}$, not only due to the addition of another observable (the LHC limit) but also because compared to the best fit point of the LEO fit, the best fit point of the LHC fit provides a worse description of $a_{\mu}$, as indicated by figures 4.3(c) and 4.3(d). The agreement gets much worse if instead of the lower limit on the Higgs mass the constraint of $m_{h}=126 \mathrm{GeV}$ is considered, as shown in Figure 4.3(e). Abandoning the unification of the Higgs mass parameters with the SUSY scalar mass parameters at $m_{\mathrm{GUT}}$ enhances the agreement between the model predictions and observations slightly, c.f. Figure 4.3(f). A much more detailed discussion of the fit results are provided in [94].

Note that the calculation of a $p$-value from the $\chi^{2} /$ d.o.f. is not performed here, as such a calculation would be spoiled by the computational limitations and the non-gaussian nature of the fit. This problem will be considered in much more detail in [104].

\subsubsection{Finetuning}

In addition to the overall agreement between measurements and predictions, the naturalness of a model is sometimes found to be of high interest and as discussed in chapter 2 it is sometimes even used to set upper bounds on the soft SUSY breaking mass parameters. Here, the fine-tuning in the CMSSM, quantified by the measure (2.83), is studied, and the implications of the nonobservation of SUSY with the LHC experiments during the $\sqrt{s}=7 \mathrm{TeV}$ run are elaborated. After that, the problem of naturalness is assessed from a different angle by studying the behaviour of the linear correlation between the fundamental parameters of the CMSSM and the NUHM1, if these models are subject to experimental contraints.

For the study of the fine-tuning measure (2.83) the spectrum has been calculated using the program SoftSUSY, in contrast to the results presented in section 4.2.1. Also, for technical reasons, the programs HiggsBounds and AstroFit are not interfaced for these studies, such that the results shown here are not completely comparable the results shown in the previous section. Only the dark matter relic density $\Omega_{\mathrm{CDM}}$ and a lower bound on the Higgs mass of $m_{h}=114.5 \mathrm{GeV}$ have been used to constrain the CMSSM. Apart from these two changes, two fits of the CMSSM (a) to the observable set LEO and (b) to the observable set LHC as discussed above have been performed.

Note also, that the study presented in this section has been performed to assess the impact of the LHC exclusion on the naturalness of the CMSSM. Some of the results shown in this section are therefore only snapshots of the full results where the less relevant parts are not shown in the plots.

\begin{tabular}{c|cccccc} 
Fit & $M_{0}[\mathrm{GeV}]$ & $M_{1 / 2}[\mathrm{GeV}]$ & $A_{0}[\mathrm{GeV}]$ & $\tan \beta$ & $M_{H}^{2}\left[\mathrm{GeV}^{2}\right]$ & $\chi^{2} /$ d.o.f. \\
\hline CMSSM LEO & $84.4_{-28.1}^{+114.6}$ & $375.4_{-87.5}^{+174.5}$ & $186.3_{-843.7}^{+831.4}$ & $14.9_{-7.2}^{+16.5}$ & - & $10.3 / 8$ \\
CMSSM LHC & $304.4_{-185.2}^{373.7}$ & $664.6_{-70.9}^{+138.3}$ & $884.8_{-974.9}^{+1178.0}$ & $34.4_{-21.3}^{+10.9}$ & - & $13.1 / 9$ \\
CMSSM LHC $+m_{h}$ & $1163.2_{-985.7}^{+1185.3}$ & $1167.4_{-513.0}^{+594.0}$ & $-2969.1_{-1234.9}^{+629.8}$ & $39.3_{-32.7}^{+16.7}$ & - & $18.4 / 9$ \\
NUHM1 LHC $+m_{h}$ & $124.3_{-16.8}^{+95.2}$ & $655.5_{-65.0}^{+218.0}$ & $-511.2_{-988.6}^{+574.7}$ & $29.4_{-7.8}^{+3.3}$ & $\left(-1.7_{-2.7}^{+0.5}\right) \cdot 10^{6}$ & $15.3 / 8$
\end{tabular}

Table 4.4: Summary of fit results [94]. Three different sets of observables, LEO, LHC and $\mathrm{LHC}+m_{h}$ have been considered, and two different models, the CMSSM and the NUHM1 have been fitted to the observable sets. 


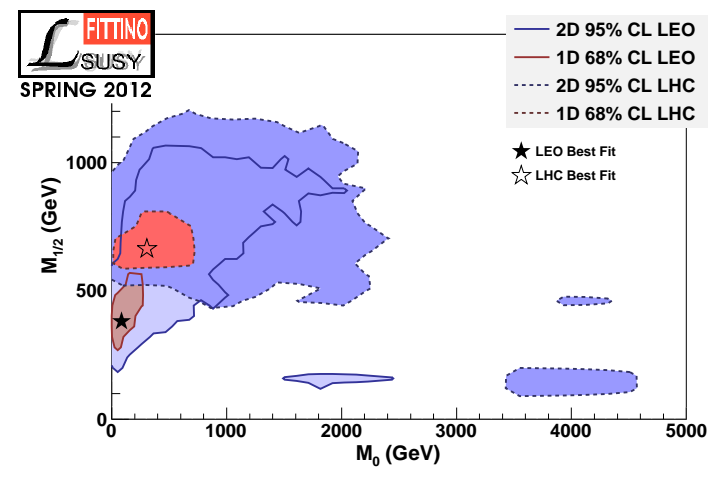

(a) CMSSM LEO vs LHC

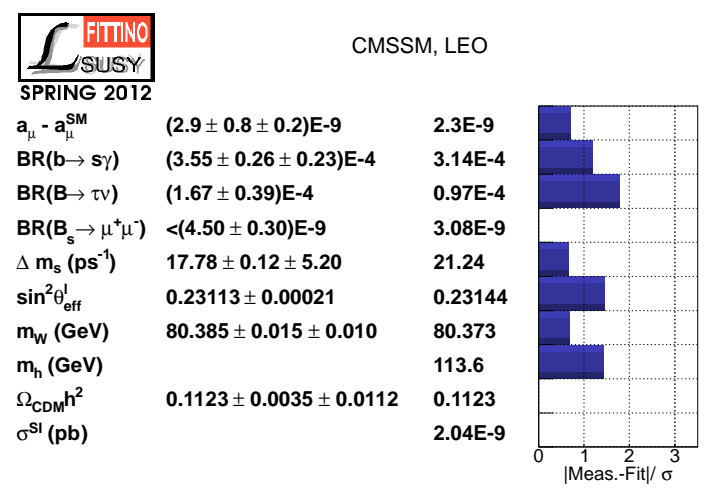

(c) CMSSM LEO

\begin{tabular}{|c|c|}
\hline $\mathcal{L}_{\text {SUSY }}^{\text {FIIINO }}$ & CMSSM, LHC \\
\hline SPRING 2012 & \\
\hline$a_{\mu}-a_{\mu}^{S M}$ & $(2.9 \pm 0.8 \pm 0.2) \mathrm{E}-9$ \\
\hline $\mathrm{BR}(\mathrm{b} \rightarrow \mathbf{s \gamma})$ & $(3.55 \pm 0.26 \pm 0.23) E-4$ \\
\hline $\mathbf{B R}(\mathbf{B} \rightarrow \tau v)$ & $(1.67 \pm 0.39) E-4$ \\
\hline $\mathbf{B R}\left(\mathbf{B}_{\mathbf{s}} \rightarrow \mu^{+} \mu^{-}\right)$ & $<(4.50 \pm 0.30) \mathrm{E}-9$ \\
\hline$\Delta \mathrm{m}_{\mathrm{s}}\left(\mathrm{ps}^{-1}\right)$ & $17.78 \pm 0.12 \pm 5.20$ \\
\hline $\sin ^{2} \theta_{\mathrm{eff}}^{1}$ & $0.23113 \pm 0.00021$ \\
\hline $\mathrm{m}_{\mathrm{w}}(\mathrm{GeV})$ & $80.385 \pm 0.015 \pm 0.010$ \\
\hline $\begin{array}{l}m_{\mathrm{h}}(\mathrm{GeV}) \\
\text { LHC }\end{array}$ & $126.0 \pm 2.0 \pm 3.0$ \\
\hline $\begin{array}{l}\Omega_{\mathrm{CDM}} h^{2} \\
\sigma^{\mathrm{Sl}}(\mathbf{p b})\end{array}$ & $0.1123 \pm 0.0035 \pm 0.0112$ \\
\hline
\end{tabular}

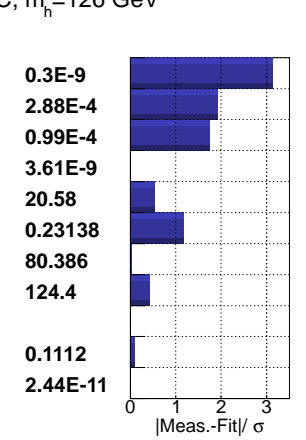

(e) CMSSM LHC $+m_{h}$

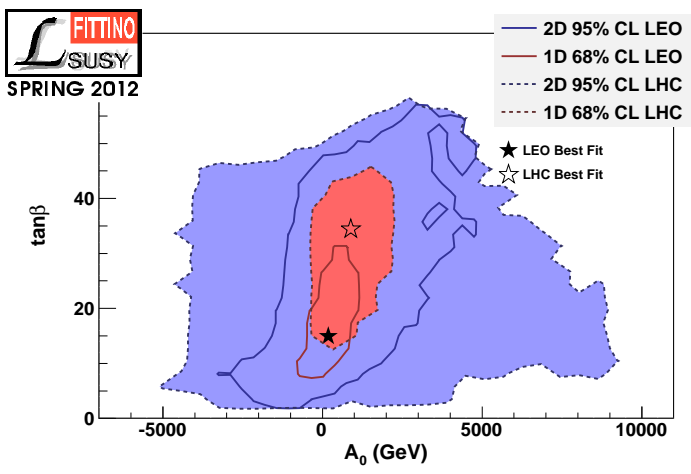

(b) CMSSM LEO vs LHC

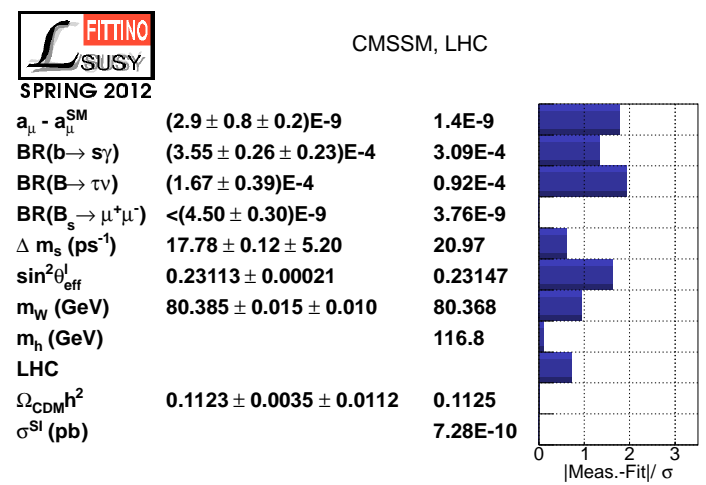

(d) CMSSM LHC

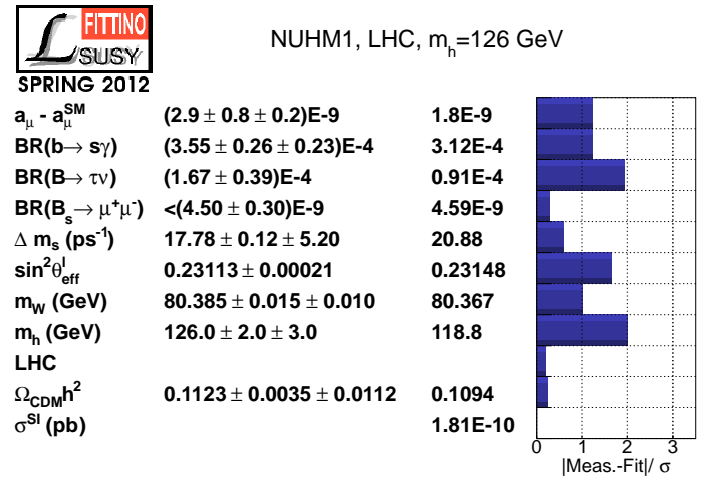

(f) NUHM1 LHC $+m_{h}$

Figure 4.3: Results of scans of the CMSSM and the NUHM1 with observables available in 2011 as published in [94].

\section{Classical Finetuning}

The problem of the naturalness of a model and a measure for the naturalness of a SUSY model have been introduced in chapter 2, c.f. equation (2.83). As discussed in that chapter, the measure $\Delta$ is sometimes used to motivate upper bounds on the masses of the superpartners. While there is no strict physical reason to reject a theory with a large value of the $\Delta$ parameter, it has still been found of interest to study the behaviour of this parameter in the region of the 


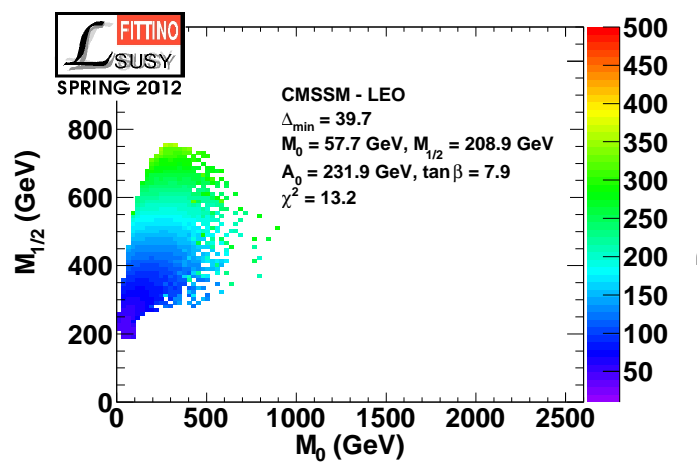

(a)

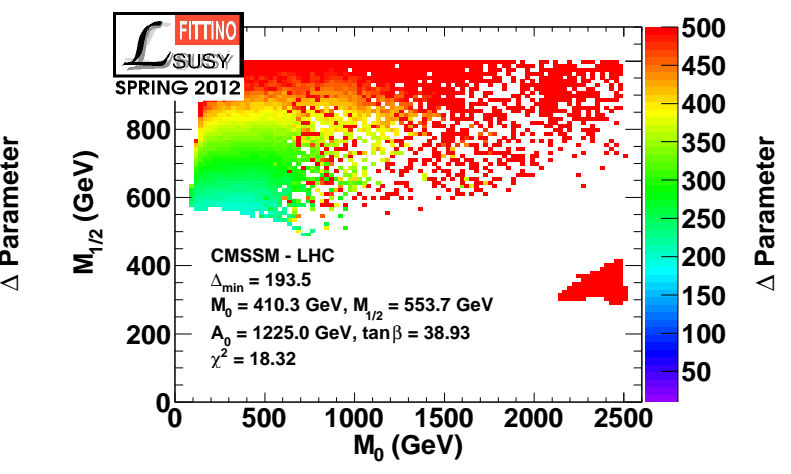

(b)

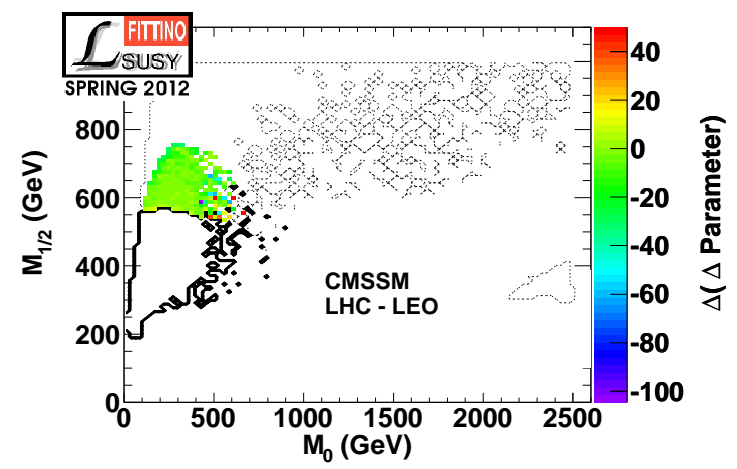

(c)

Figure 4.4: The minimal amount of fine-tuning as a function of $M_{0}$ and $M_{1 / 2}$, where $A_{0}$ and $\tan \beta$ are profiled [94], for (a) the pre-LHC fit and (b) the LHC fit. (c) shows the difference, (b) - (a), between both fits, which is mostly close to vanishing in the overlap of the $95 \%$-CL regions. The last bin on the z-axis in (a), (b), and (c) contains all points with $\Delta>500$. The smallest values for the $\Delta$ parameter are 39.7 (193.5) for the pre-LHC (LHC) fit.

parameter space that is preferred by recent observations. It is in particular the impact of the LHC limits that is of high interest in this context.

Two scans of the CMSSM parameter space have been performed using SoftSUSY as a spectrum calculator, where the model has been constrained using the LEO with and without the LHC limits and the $\Delta$ parameter (2.83) with $\left\{a_{i}\right\}=M_{0}, M_{1 / 2}, A_{0}, M_{3}, \mu$ and $h_{t}$, which is the top Yukawa coupling, has been determined at each point. The results of the fit are shown in Figure 4.4. The plots in figures 4.4(a) and 4.4(b) show the minimal value of $\Delta$ in the $M_{0}-M_{1 / 2}$ plane where $A_{0}$ and $\tan \beta$ have been profiled: In each bin, using all points in the MCMC with $\Delta \chi^{2}<5.99$ with respect to the minimum, with $M_{0}$ and $M_{1 / 2}$ within the bin boundaries, and with any value of $A_{0}$ and $\tan \beta$, the minimal value of $\Delta$ is shown.

In the 2-dimensional $2 \sigma$ area of the LEO fit it has been found that $\Delta \geq 39.7$ for all points. This minimal value of $\Delta$ is sometimes still considered to be acceptable for a natural theory. In general the larger $M_{0}$ and $M_{1 / 2}$ get, the larger gets the $\Delta$ parameter, such that at the boundaries of the 2-dimensional $2 \sigma$ region values of $\Delta \gtrsim 300$ are found, which is usually not considered to be natural any more. 
The inclusion of the LHC limits decreases the overall quality of the fit, thus allowing for a wider range in $A_{0}$ and $\tan \beta$ for larger values of $M_{0}$ and $M_{1 / 2}$, where the LHC experiments are not yet sensitive. The smallest value of $\Delta$ found in the 2-dimensional $2 \sigma$ area is given by $\Delta \geq 193.5$, and in the $95 \%$-CL region points with $\Delta>500$ are easily found. These values are significantly larger than what is commonly considered to be natural. As for the LEO fit, larger values of $\Delta$ are observed at larger values of the mass parameters.

There is a small overlap between the 2-dimensional $2 \sigma$ regions of the LEO and the LHC fits. In this region the minimal amount of fine-tuning can be compared between both fits. This is done in Figure 4.4(c), where the absolute difference of the profiled values of $\Delta$ between the LHC and the LEO fit is shown. A negative (positive) entry in this histogram indicates that with the inclusion of the LHC limits a lower (higher) amount of fine-tuning is allowed at that point. The solid (dashed) contours depict the regions in the $M_{0}-M_{1 / 2}$ plane that are part of the $95 \%$-CL area in the LEO (LHC) fit, but not in the LHC (LEO) fit. In the overlap region, the minimal amount of fine-tuning is similar for both fits, although in general the LHC fit tends to allow for slightly smaller values, here. This can be explained by the widened range in $A_{0}$ and $\tan \beta$ due to the overall worse quality of the fit.

In summary, if the limits on the CMSSM parameter space set by the ATLAS experiment using two years of LHC data are considered in a fit of the CMSSM, the minimal amount of fine-tuning in the region that is preferred by all relevant observables is increased from $\Delta \geq 39.7$ to $\Delta \geq 193.5$; this means that with the non-observation of CMSSM-like supersymmetry at the LHC after two years, the measure for naturalness of the CMSSM is pushed above the boundary below which a model is still considered to be acceptably fine-tuned, which is usually given by $\Delta \lesssim 100$.

\section{A Different Approach to Finetuning}

Although it has been criticised and modifications to it have been proposed (see [135], for instance), from a theoretical point of view, the fine-tuning measure $\Delta$ has many merits. From an experimental point of view it is however flawed in a fundamental way. Technically, with the measure $\Delta$ the naturalness of a model is quantified based on a single, arbitrarily chosen observable $O$, such that the amount of fine-tuning that is assigned to some point in the parameter space of that model can differ significantly if a different observable $O^{\prime}$ was chosen. A large value of $\Delta$ renders a model unnatural because due to the large dependence of the observable $O$ on at least one parameter, a large change in $O$ due to a small variation of that parameter has to be cancelled by variations of the other parameters in order to restore agreement between the model prediction and the measured value of $O$.

If the naturalness of a model is to be quantified by such considerations, it would seem more interesting to define a measure for fine-tuning based on all relevant observables rather than a single observable; in particular the uncertainty on the measurements should be taken into account for such a calculation. As an additional information on how much the parameters of a model have to be tuned with respect to each other, a measure based on the correlation of the fundamental parameters in a fit has therefore been proposed in [94]. The approach will be briefly described here.

For any point $P$ in the parameter space, the agreement between predictions and observations is described by the value of $\chi^{2}(P)$ and the difference in the agreement between that point and any other point $P^{\prime}$ can be described by the quantity $\Delta \chi^{2}\left(P, P^{\prime}\right)=\chi^{2}(P)-\chi^{2}\left(P^{\prime}\right)$. Although there is a certain amount of arbitrariness in this definition, it can be claimed that all points $P^{\prime}$ with $\Delta \chi^{2}\left(P, P^{\prime}\right)<0.001$ describe the set of measurments used in the fit equally well as the 
point $P$. While a priori the fundamental parameters of the CMSSM/NUHM are independent, a variation in one of the parameters $P_{i}$ may force a correlated variation in one or more of the other parameters $P_{j}$ in order to keep the agreement between the predictions and the measurements at the same level. In this context a model is considered to be more fine-tuned, if after a change in the parameter $P_{i}$ there is less freedom in varying one or more of the parameters $P_{j}$ without altering the level of agreement between predictions and observations. In the following a measure for this freedom is defined.

Let $P$ be some point in the parameter space that describes the available measurements with a quality of fit $\chi^{2}(P)$. Let $\left\{P^{\prime}\right\}$ be all distinct points in the scan with $\Delta \chi^{2}\left(P, P^{\prime}\right)<0.001$. For any quantity $Q=Q(P)$, in the following the average $\langle Q\rangle$ and the spread $\sigma_{Q}$ is defined as

$$
\begin{aligned}
\langle Q\rangle(P) & =\frac{1}{\left|\left\{P^{\prime}\right\}\right|} \sum_{\left\{P^{\prime}\right\}} Q\left(P^{\prime}\right), \\
\sigma_{Q}^{2}(P) & =\frac{1}{\left|\left\{P^{\prime}\right\}\right|} \sum_{\left\{P^{\prime}\right\}}\left(Q\left(P^{\prime}\right)-<Q>(P)\right)^{2} .
\end{aligned}
$$

While for convenience the dependence of the average (4.7) and the spread (4.8) on the point $P$ will be omitted, it must be stressed that at all times these quantities have to be considered as a function of the position in the parameter space. With these definitions it is possible to measure the correlation between two parameters $P_{i}$ and $P_{j}$ using Pearson's product-moment correlation coefficient:

$$
\varrho_{i j}(P)=\left\langle\frac{\left(P_{i}-<P_{i}>\right) \cdot\left(P_{j}-<P_{j}>\right)}{\sigma_{P_{i}} \sigma_{P_{j}}}\right\rangle
$$

where in the following as for the average and the variance, the dependence on $P$ will be omitted. If the model under study has $n$ parameters, at each point a total of $\left(n^{2}-n\right) / 2$ correlation factors are evaluated. The quantity $\varrho_{\max }$ is then defined as

$$
\varrho_{\max }=\max _{i j}\left(\left|\varrho_{i j}\right|\right) \text {. }
$$

At or near the true minimum of a fit, the maximum correlation coefficient $\varrho_{\max }$ will naturally be close to 1 for a highly constrained model, as near to the minimum there is little freedom in varying any of the parameters. Depending on the model and the measurements it may however acquire large or small values over the full parameter space that is allowed; the actual values will depend on how finely the parameters have to be tuned to each other in order to keep the agreement between predictions and data at one level.

While the classical measure for fine-tuning, the $\Delta$ parameter, is always a very local quantity, the maximum correlation coefficient $\varrho_{\max }$ may describe features of the scanned parameter space at very different scales. For small values of $\chi^{2}$, the range in each parameter that is covered by the set $\left\{P^{\prime}\right\}$ will usually be small, while for larger values of $\chi^{2}$, the set will also cover a larger range on each axis. Therefore the measure (4.10) will describe local features of the allowed parameter space for small $\chi^{2}$ values and more global features of the model parameter space for larger values of the $\chi^{2}$. Also, this measure is based on the linear correlation only, and if the tuning between parameters needs to be done in a more complicated way, the interpretation of this measure as an amount of fine-tuning is diluted. It is nevertheless a reasonable starting point for a phenomenologically motivated measure for fine-tuning. In order to allow for a meaningful interpretation of the measure (4.10) it has not been calculated for any point where $\left|\left\{P^{\prime}\right\}\right|<6$ note however that for most points $\left|\left\{P^{\prime}\right\}\right| \sim 10^{3}$. 
In order to define a baseline reference, a scan of the CMSSM parameter space has been performed using only a minimum set of requirements. No measurements have been used for this scan, but it was required that (a) the lightest neutralino is the LSP, (b) there is consistent radiative electroweak symmetry breaking, and (c) there are no tachyons. In order to have a meaningful reference value, the correlation coefficients between any pair of parameters have been calculated in a rectangular subset of the full parameter space, the boundaries of which have been defined by the maximum and minimum value of each parameter within the 95\%-CL region of the CMSSM LHC fit. These correlation coefficients have been found to be smaller than 0.2 , against which the following results should be compared.

The behaviour of the measure (4.10) in the CMSSM in the 2-dimensional $2 \sigma$ area using the LEO set and the LHC set is shown in Figure 4.5. The plots show the minimal value of $\varrho_{\max }$ as a function of $M_{0}$ and $M_{1 / 2}$, where the model parameter $A_{0}$ and $\tan \beta$ have been profiled. Figure 4.5(a) shows the behaviour of the profiled $\varrho_{\max }$ in the $M_{0}-M_{1 / 2}$ plane for the fit using all LEO but not the LHC limits. A minimum correlation factor of $\varrho_{\max }^{\min }=0.62$ is found, which is well above the reference of 0.2 . The tuning between the parameters is increased for increasing values of the mass parameters $M_{0}$ and $M_{1 / 2}$ in this model.

If the LHC limits are included in the fit, the correlation factors behave as shown in Figure $4.5(\mathrm{~b})$. The quality of the fit has significantly decreased, and the 2-dimensional $2 \sigma$ region is significantly enlarged with respect to the LEO fit. The minimal correlation coefficient is found to acquire a value of $\varrho_{\max }^{\min }=0.29$ in this case, which is small compared to the LEO fit, but still larger than the reference value. The significant difference between the LEO and the LHC fit can be explained by the wider ranges in all parameters that contribute to the 2-dimensional $2 \sigma$ region in the LHC fit. This is particularly emphasised in Figure 4.5(c), where the difference between the minimal correlation coefficient for the LEO and LHC fits is shown in the overlap of the 2-dimensional $2 \sigma$ areas.

The behaviour of the measure (4.10) has also been investigated for slightly different scenarios. For the following results, instead of SoftSUSY, the package SPheno has been used as a spectrum calculator again and the AstroFit package has been included for the calculation of the $\chi^{2}$ at each point. The observable set LHC $+m_{h}$ has been used to constrain the CMSSM and the NUHM1. Two different fits of the CMSSM parameters have been performed; one with the standard set of parameters, $M_{0}, M_{1 / 2}, A_{0}$ and $\tan \beta$, and one where this set has been augmented by the mass of the top quark, $m_{t}$, which has been allowed to vary around its central value according to the uncertainty on its measured value, $\sigma_{m_{t}}=1.3 \mathrm{GeV}$.

The results of this study are shown in Figure 4.6, where all parameters except for $M_{0}$ and $M_{1 / 2}$ are profiled. The quality of the fit decreases, and the range of the parameters in the 2 -dimensional $2 \sigma$ areas is significantly increased. Minimal correlation factors of $\varrho_{\max }^{\min }=0.59$ for the CMSSM fit with a fixed top mass, $\varrho_{\max }^{\min }=0.70$ for the CMSSM fit with a floating top mass, and $\varrho_{\max }^{\min }=0.45$ for the NUHM1 are observed. The increase in the minimal correlation factors observed by adding the top mass as an additional parameter to the fit can have two reasons - first, at each point the new maximum correlation factor can be one of the correlation coefficients describing the correlation between the top mass parameter and one of the SUSY breaking parameters; second, a set of points that are excluded in the fit with a fixed top mass parameter can be re-introduced into the $95 \%$-CL region if by varying the top mass parameter the $\chi^{2}$ for such a point can be reduced sufficiently. For the behaviour of $\varrho_{\max }^{\min }$ observed here, it is the latter effect that has been found to be dominant.

As discussed and shown above, the value of $\varrho_{\max }$ calculated at a given point may change significantly, if points with worse agreement between model predictions and observation are reintroduced in the 95\%-CL region by adding another observable to the fit. In the above studies, 


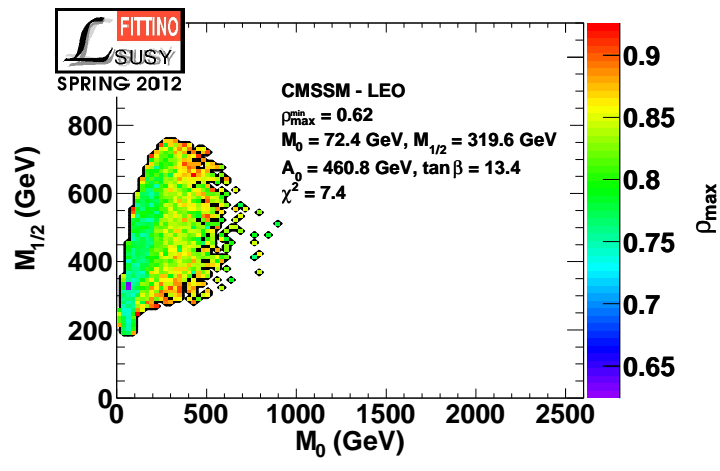

(a)

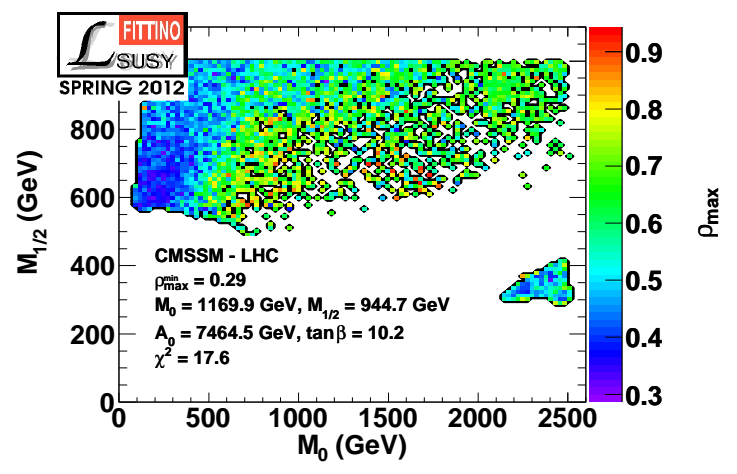

(b)

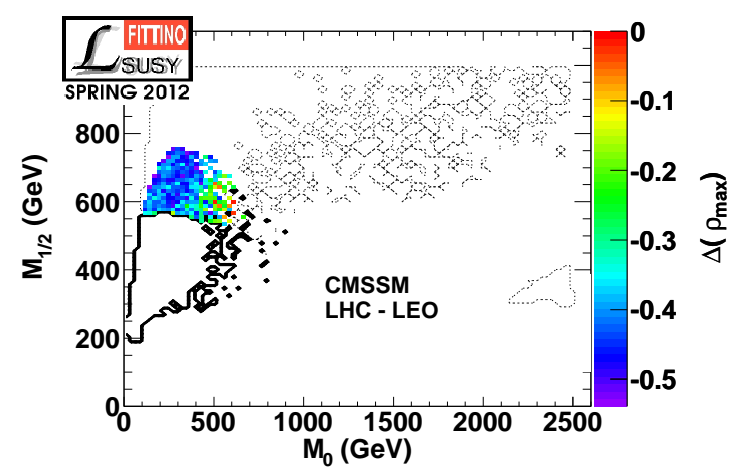

(c)

Figure 4.5: Minimum value of $\varrho_{\max }$ as a function of $M_{0}$ and $M_{1 / 2}$, where $A_{0}$ and $\tan \beta$ are profiled [94]. (a) shows the minimum correlation in the 95\%-CL region of the pre-LHC fit, (b) shows the minimum correlation in the 95\%-CL region of the LHC fit, (c) shows the difference of both distributions. For nearly all values of $M_{0}$ and $M_{1 / 2}$, the pre-LHC fit shows a larger minimum correlation. The smallest values of $\varrho_{\max }$ are $0.62(0.29)$ for the pre-LHC (LHC) fit.

this has been taken into account by considering all points in the 95\%-CL region and minimising $\varrho_{\max }$ over all points in one bin. It is also of interest to study the behaviour of the correlation factors $\varrho_{\max }$ if for each bin in the $M_{0}-M_{1 / 2}$ plane only the point describing the data best is considered. For the fits of the CMSSM with fixed and free top mass this is shown in Figure 4.7. Figure 4.7(a) shows the correlation coefficient $\varrho_{\max }$ in the $M_{0}-M_{1 / 2}$ plane for the points with the smallest $\chi^{2}$ in each bin for the fit of the CMSSM with the top mass fixed, while Figure 4.7(b) shows the same for the fit where the top mass parameter has been allowed to fluctuate around its central value. The difference between both fits is shown in Figure 4.7(c). A negative (positive) entry in this histogram indicates that with a free (fixed) $m_{t}$ a lower correlation between the fundamental parameters is found. In particular close to the minimum in $\chi^{2}$, a floating $m_{t}$ allows for a smaller maximum correlation factor, whereas in other regions of the allowed parameter space the maximum correlation increases if $m_{t}$ is set free. White areas in these plots indicate that at the corresponding point with the lowest $\chi^{2}$ the cardinality of the set $\left\{P^{\prime}\right\}$ was smaller than 6 , or that the point was included in the $95 \%$-CL region of one fit, but not in the $95 \%$-CL region of the other fit. 


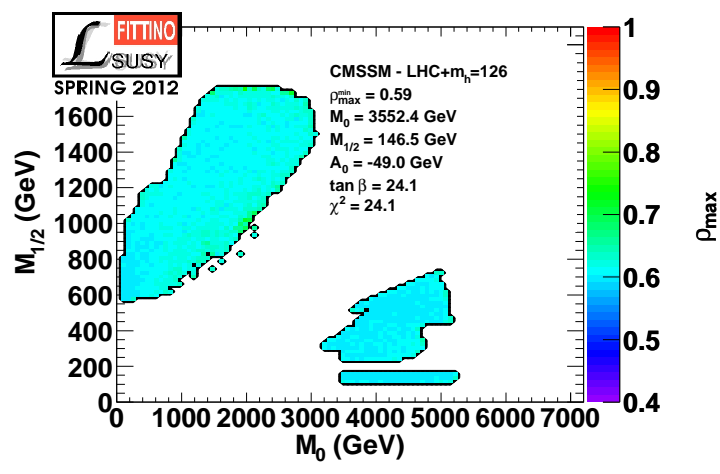

(a)

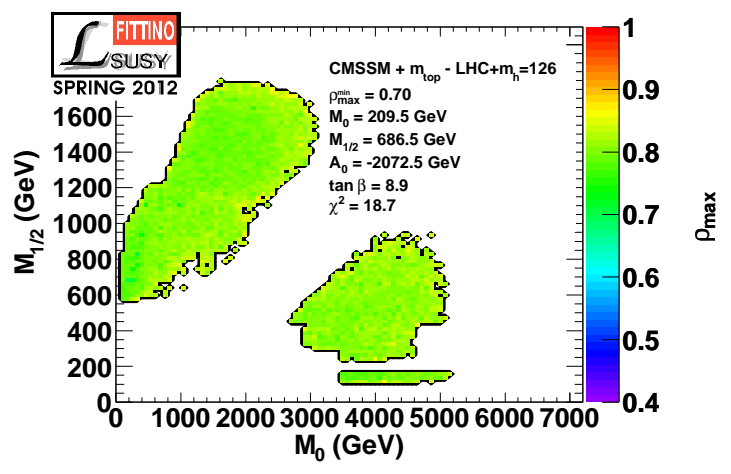

(b)

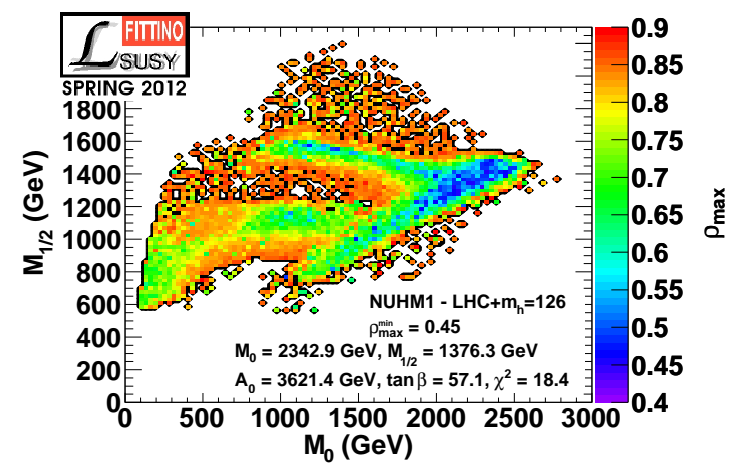

(c)

Figure 4.6: Minimum value of $\varrho_{\max }$ as a function of $M_{0}$ and $M_{1 / 2}$, where $A_{0}$ and $\tan \beta$ are profiled [94]. (a) and (b) show the minimum correlation for a fit of the CMSSM model with a fixed (free) $m_{t}$. For all points, the minimum correlation increases for a free $m_{t}$. The minimum value for $\left[\varrho_{\max }\right]_{\min }$ is $0.59(0.70)$ for the fit with fixed (free) $m_{t}$. In addition, (c) shows the $\varrho_{\max }$ as a function of $M_{0}$ and $M_{1 / 2}$ for a fit of the NUHM1 model, where $A_{0}, \tan \beta$ and $m_{H^{0}}$ are profiled. The minimum value of $\varrho_{\max }$ for this fit is 0.45 . All fits use $m_{h}=126 \pm 3 \mathrm{GeV}$.

\subsection{Summary of Results}

A study of the CMSSM and NUHM1 after two years of LHC operation has been presented. The non-observation of any excess with respect to the SM in the searches for SUSY with jets, $E_{T}$ and no leptons in the final state pushes the lower bounds on the CMSSM/NUHM1 mass parameters for the superpartners to large values, at which the available measurements from the low energy sector are significantly worse described, in particular if the existence of a Higgs boson with a mass of $m_{h}=126 \mathrm{GeV}$ is considered. The relaxation of unification assumptions can restore a slightly better agreement between all observations and the predictions of a minimal SUSY model.

While the CMSSM/NUHM1 with small mass parameters is in contradiction to the results of the direct searches at the LHC, it is in particular the measurement of $a_{\mu}$ that prefers these small mass parameters. Therefore it seems interesting to relax even more of the unification relations in the CMSSM/NUHM1; in particular the coupling of the mass parameters for the coloured 


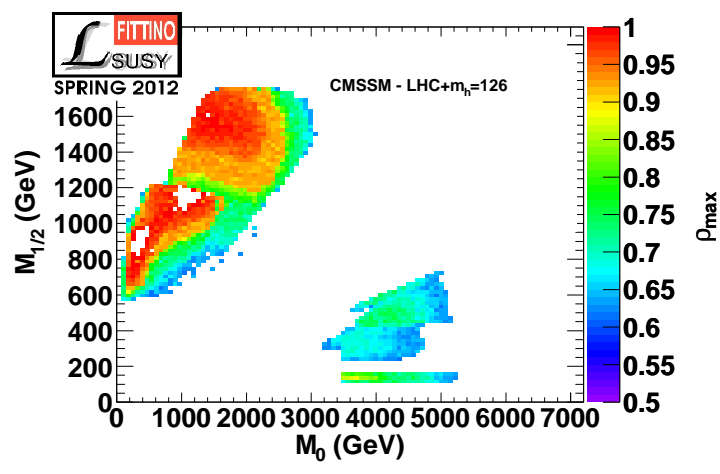

(a)

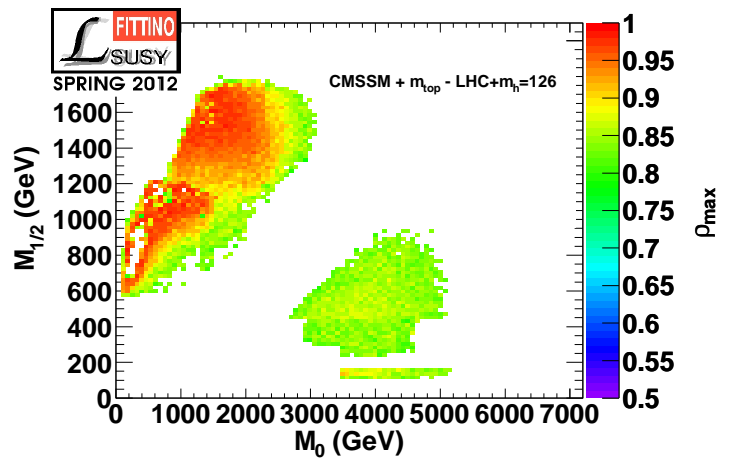

(b)

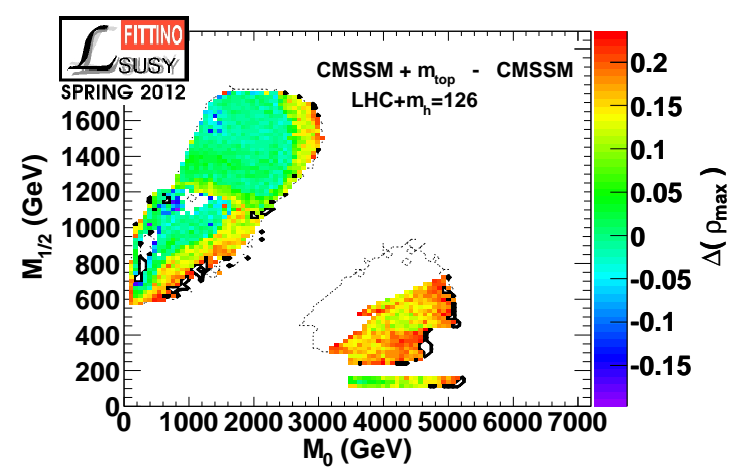

(c)

Figure 4.7: The value of $\varrho_{\max }$ as a function of $M_{0}$ and $M_{1 / 2}$ at the point with the lowest $\chi^{2}$, where $A_{0}$ and $\tan \beta$ are profiled [94]. (a) shows the minimum value for a fit of the CMSSM model where $m_{t}$ is fixed, (b) shows the minimum value for a fit of the CMSSM model where $m_{t}$ is free. (c) shows the difference of the two distributions. Near the best-fit point, the minimum of $\varrho_{\max }$ is increased if $m_{t}$ is fixed, while at the boundaries of the 95\%-CL region the minimum value is decreased. Both fits are constrained by $m_{h}=126 \pm 3 \mathrm{GeV}$.

sparticles to the mass parameters of the colourless sparticles seems troubling - for instance, $a_{\mu}$ is particularly sensitive to the mass parameters of the superpartners of the muon and the muon-neutrino. Although it is by far not an exact statement, the ATLAS searches that are most sensitive to the CMSSM tend to exclude coloured sparticles with a small mass, while for instance $a_{\mu}$ tends to prefer smaller mass parameters for the smuon and the muon-sneutrino, for instance. It is therefore of interest to search for SUSY not only in final states with jets, but also in final states with leptons, which may be more sensitive to a model with light gauginos/Higgsinos and sleptons, but heavy squarks and gluinos. In the following chapters, some aspects of such a search are detailed. 



\section{Measurements and Application of ATLAS Lepton Trigger Efficiencies}

Compared to baryons, leptons are created seldomly in the hadronic environment at the LHC. Furthermore they are exclusively produced by electroweak processes, some of which are amongst the processes that are to be studied at the LHC. The existence of a charged lepton candidate in an event, typically with a large transverse momentum, is therefore one of the primary signatures that is used to trigger on events and select them for further processing. For the calculation of cross sections as well as the correct normalisation of MC to data it is of importance to measure the efficiency of the triggers used in the respective analysis. There are several methods that can be used to determine the efficiency of lepton triggers in a data-driven way without relying on $\mathrm{MC}$, and there are several methods on how a measurement of the trigger efficiency is embedded into a physics analysis.

In this chapter, after a short overview of processes with final states including leptons in both the SM and in the MSSM, and a brief introduction to trigger efficiencies, the application of lepton trigger efficiencies in a physics analysis is discussed. Two different methods, the scale factor approach and the re-weighting approach, are compared and some extensions to a software package that was developed for applying the latter approach [136] are presented. Finally, the performance of the ATLAS muon-trigger system during the $\sqrt{s}=7 \mathrm{TeV}$ run is studied.

\subsection{Leptonic Final States at the LHC}

Leptons at the LHC are produced exclusively via the electroweak interaction, either in the hard process or in the weak decay of strongly interacting particles or bound states of these. It is for this reason that in the SM, if within kinematic limits, the branching fractions of the leptonic decays of the $W$ and the $Z$ bosons determine the relative amount of events with one, two or more leptons in the final state for a given hard process. While the non-leptonic final states usually have a larger branching fraction and an analysis with leptonic final states is usually performed using smaller statistics than an analysis targeting non-leptonic final states, for most analyses at the LHC the number of background events in the latter case is much higher. Also, leptons can be observed and their four-momentum can be measured directly, while for quark final states the observed objects are jets, such that transfer functions are needed to reconstruct the event properties at the parton level.

In models beyond the SM there are other ways to produce leptons. In SUSY models, these are usually produced via the Yukawa-like and gauge interactions of the gauginos and sleptons. There are regions of the SUSY parameter space in which the leptonic branching fractions of the SM gauge bosons are also of importance; but in SUSY models it is often the difference in the masses of sleptons and squarks that determines the fraction of events with leptons in the final state.

In this and in the following chapters, the term lepton usually refers to the charged light leptons in the SM, $e$ and $\mu$. While the $\tau$ is technically still a lepton, it plays a special role amongst the SM leptons as it decays relatively quickly, while both the $e$ and $\mu$ have an average lifetime that is 
larger than the detector scale, $\gamma_{\mathrm{LHC}} c \tau_{\tau}<10 \mathrm{~m}<\gamma_{\mathrm{LHC}} c \tau_{e / \mu}$. Universality between the first two lepton generations is a usually a good approximation, such that in case the flavour of the leptons in the final state does not matter, the charged leptons will be referred to $l^{ \pm}$, where the charge will sometimes be omitted. Also, the neutral leptons will be referred to as $\nu_{l}$ - anti-neutrinos are referred to as $\bar{\nu}_{l}$. Leptons that originate from the weak decay of a hadron within a jet are usually referred to as non-prompt or fake leptons, whereas leptons that are created in the hard interaction are usually referred to as prompt or real leptons.

\subsubsection{Final States with Leptons in the Standard Model}

In the SM the 'easiest' way to produce high- $p_{T}$ leptons in final states at the LHC is the production and decay of the massive electroweak gauge bosons,

$$
p p \rightarrow Z X \rightarrow l^{+} l^{-} X \quad \text { and } p p \rightarrow W^{ \pm} X \rightarrow l^{ \pm} \nu_{l} X .
$$

The branching fraction $\mathrm{BR}\left(Z \rightarrow l^{+} l^{-}\right)$is about $6.6 \%$, while the branching fraction $\mathrm{BR}\left(W^{+} \rightarrow\right.$ $l^{+} \nu_{l}$ ) is roughly 21.3\% [34]. Despite the small branching fraction, the production of $Z$ bosons with leptons in the final state is still one of the most important processes that is studied at the LHC. Of particular interest is the production of an electroweak boson in association with one or more jets, where the leptons in the final state are used to identify and select such events. Also, as the mass of the $Z$ boson is known very precisely (Table 2.2), the process $Z \rightarrow e^{+} e^{-}$can be used to calibrate electromagnetic calorimeters for both electrons and photons. Due to the clean signatures and the well known couplings of the $Z$ boson, it is also possible to use the process $Z \rightarrow l^{+} l^{-}$in order to estimate the number of events where the $Z$ boson decays invisibly - in the SM usually $Z \rightarrow \overline{\nu_{l}} \nu_{l}$, which is an important background to some searches for new physics.

Besides providing an interesting signature by itself, the $W$ boson plays a special role in the distribution of the number of leptons in final states of events including one or more top quarks. The top quark decays into a bottom quark and a $W$ boson with a branching fraction of nearly $100 \%$ [34], as shown in Figure 5.1(a). The fraction of events with zero, one or two isolated high- $p_{T}$ leptons is then given by the product of the branching ratios of the $W$ boson.

In the searches for the Higgs boson, light leptons also play an important role. While the decay of the Higgs boson into two light leptons is highly suppressed by the small Yukawa couplings in the first two generations, the light leptons play a keyrole in the reconstruction of events in which a Higgs boson decays into the massive electroweak gauge bosons. For instance, the decay $H \rightarrow Z Z^{*} \rightarrow l^{+} l^{-} l^{+} l^{-}$has a very small branching fraction, but provides a very clean signature.

\subsubsection{Searches for Supersymmetry in Leptonic Final States}

Depending on the mass hierarchy and the mass scale of the supersymmetric particles, there are numerous scenarios in the MSSM where final states that provide the highest sensitivity contain one or more leptons. If the superpartners of the coloured SM particles are light enough, strong production of squarks and/or gluinos will typically dominate at the LHC. If the mass hierarchy allows for decays of squarks into the heavier neutralinos and charginos, there is a reasonable probability to observe leptons - in addition to high- $p_{T}$ jets - in the final state. If, on the other hand, the squark masses are too large such that the coloured superpartners cannot be produced directly at the LHC, but the gauginos and sleptons are not too heavy, there is a good chance to discover supersymmetry in final states with two or more isolated leptons and a small number of low- $p_{T}$ jets.

If squarks and gluinos are not too heavy, searches for SUSY with one lepton typically target hard processes where squarks are either produced directly or appear as the decay products of 

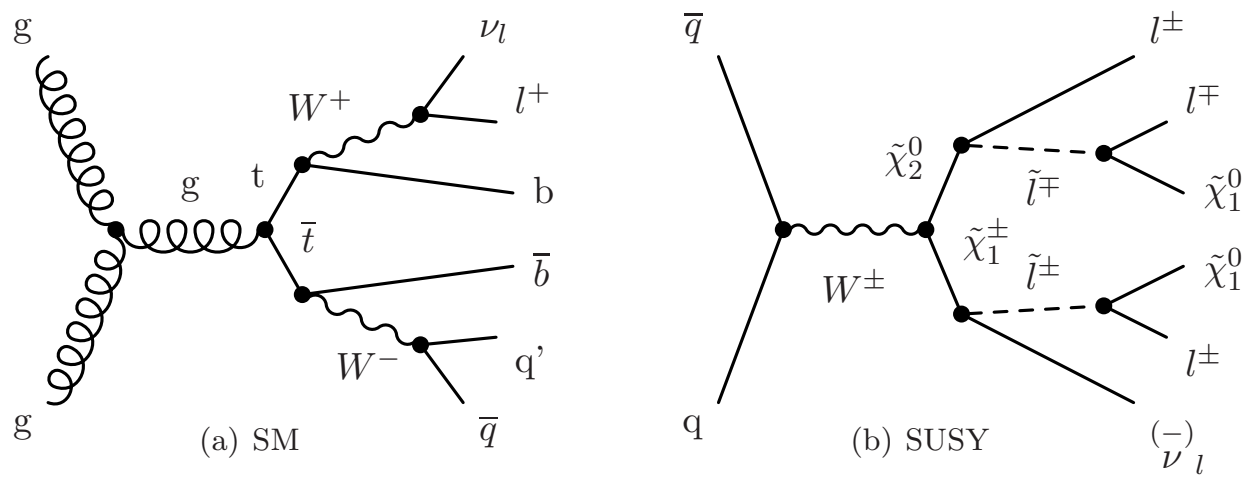

Figure 5.1: Examples for the production and decay of particles into final states with leptons. In the SM the production and decay of top quark pairs as shown in (a) is one of the processes that are studied at the LHC. It is the decay of the two $W$ bosons that defines the number of leptons in the final states. In SUSY models, the direct production and decay of gauginos can lead to final states with high lepton multiplicities, as shown in (b).

gluinos, and decay further via a chargino. Depending on the scenario several signal regions have been proposed. In the mSUGRA/CMSSM model the total missing transverse energy as well as the effective mass (for which there are different definitions) of an event have been found to be useful variables for the definition of a signal region. Compared to SM background, in a SUSY event the $E_{T}$-spectrum usually will tend to be harder. The same applies to the effective mass, which is always closely related to and sometimes defined as the scalar sum of the transverse momenta of all signal leptons and signal jets in an event.

While it is also possible to have two or even more leptons in final states from the strong production of supersymmetric particles, searches with two or more leptons in the final state also target scenarios in which the squarks and gluinos are too heavy for direct production at the LHC. If in such a case the sleptons and gauginos are not too heavy, these can be produced in pairs. Searches for SUSY in final states with two leptons usually target the direct production of sleptonand chargino-pairs, which can decay via sleptons or the electroweak gauge bosons. Searches for SUSY in final states with three leptons target the associate production of a neutralino with a chargino as shown in Figure 5.1(b), while in RPC scenarios SUSY searches in final states with four or more leptons may be sensitive to the direct production of neutralino pairs, which will be discussed in the next chapter.

\subsection{Trigger Efficiencies and Statistics}

As a basis for the following sections, the definition of a trigger efficiency as used in this thesis and the statistical interpretation of a measurement of such an efficiency are briefly described here.

\subsubsection{Definition of a Trigger Efficiency}

A trigger is designed to quickly select events based on well defined event characteristics, or signatures. As at the LHC there is usually very little time to decide if an event should be saved for further processing or not, the trigger takes its decision by evaluating a trigger hypothesis test on coarse measurements. If an event passes the hypothesis test it is selected for further processing, and these measurements are refined such that the final properties of an event in a 
physics analysis may differ from the properties based on which the trigger decision was taken. It is therefore possible that a trigger does not select all events that it is designed to select (inefficiency), or that it selects events that is is not supposed to select (fake efficiency).

There are two different types of triggers in general, those that select events based on a global property of an event, such as the missing transverse energy or the total scalar energy in an event, and those that select events based on the existence of one or more objects of a certain type in an event, such as muons or electrons, and the definition of the efficiency for both triggers is slightly different. For triggers evaluating global event properties, the true efficiency is defined as the fraction of events that are selected by that trigger, i.e.

$$
\varepsilon_{\mathrm{E}}=\lim _{N_{E} \rightarrow \infty} \frac{K_{E}}{N_{E}},
$$

where $N_{E}$ is the total number of events and $K_{E} \leq N_{E}$ is the number of events that pass the trigger in question. The trigger efficiency $\varepsilon_{E}$ is usually not a constant, but it is a function of one or more variables. Naturally it will depend on the event properties that are used to evaluate the hypothesis of the particular trigger, but it may as well depend on other variables indirectly. Note that the true efficiency of a trigger will usually not be known exactly, as it has to be measured from a finite number of events - it can usually not be calculated from the detector specifications. This will be enlarged upon in section 5.2.3.

For the second type of trigger, which selects events based on the abundance of objects in an event and their properties, the efficiency is defined in a slightly different way. One of the most prominent examples is a muon trigger, which in the simplest case is defined by a $p_{T^{-}}$ threshold: An event is selected by a muon trigger if there is at least one muon in the event with a transverse momentum larger than that threshold. If the efficiency of a muon trigger was defined as a fraction of events, like equation (5.2), it would depend on the number of muons in an event, as well as on some properties of all muons in that event, making it a function of a huge number of variables. Instead, it seems more natural to define the efficiency of such a trigger per object, i.e.

$$
\varepsilon_{O}=\lim _{N_{O} \rightarrow \infty} \frac{K_{O}}{N_{O}},
$$

where $N_{O}$ is the number of objects of a certain type, and $K_{O}$ is the number of objects passing the trigger hypothesis in question. This definition is possible in ATLAS, because the decision for triggers based on single objects is evaluated per object rather than per event. The efficiency per event can - under certain assumptions - be calculated from the single object efficiencies (5.3), as will be discussed later in this chapter.

In the following, only such object based triggers - mostly muon triggers - are considered. To finalise the definition of the efficiency of such a trigger one more clarification is needed. In the following, the efficiency of an object based trigger is always the efficiency with respect to the object reconstruction. This means that in equation (5.3) the number $N_{O}$ is the number of reconstructed objects after full reprocessing of the event, and the number $K_{O}$ is the number of reconstructed objects after full reprocessing that passed the trigger hypothesis in question. The number $K_{O}$ is determined by a procedure referred to as trigger matching. Usually only reconstructed objects of the same type as the trigger is designed to be sensitive to are considered, rendering the fake efficiency meaningless in this context. 


\subsubsection{Trigger Matching}

The trigger system in ATLAS uses look-up tables and fast reconstruction algorithms to evaluate the test hypothesis of the trigger items in the trigger menu during data taking. The reconstructed objects at the EF level (online objects) are distinct from the objects that are reconstructed for the offline analysis (offline objects), and it is in particular differences in the used calibration and alignment constants that can lead to substantial deviations between the reconstructed properties of the online object and the offline object that have been reconstructed from the same real physics object. The trigger efficiencies are usually considered as a function of the properties of the offline objects and of the event properties calculated using the offline reconstruction. As outlined above, the efficiency of a muon trigger is calculated with respect to offline muons; this means that for the calculation of a muon trigger efficiency it needs to be tested if an offline muon has passed the trigger hypothesis of the trigger in question. This is done by matching offline muons to online muons.

In order to perform such a matching, the angular distance measure $\Delta R$ is used. An offline muon is then considered to pass some trigger $A$, if

- there is an online muon reconstructed by the trigger algorithm in question passing the hypothesis of that trigger within $\Delta R<0.15$,

- there is no second online muon reconstructed by the trigger algorithm in question that matches the offline muon better in terms of $\Delta R$, and if

- there is no second offline muon that matches the same online muon better in terms of $\Delta R$.

This simple matching algorithm is not completely safe to fail. In complicated environments with many close-by muons it may be necessary to refine the matching procedure, introducing a global distance measure that takes into account all possible combinations of online-offline pairs. For the usual purposes it has nevertheless been found to work with an acceptable performance such that it can safely be used here.

An important part of a muon trigger hypothesis in ATLAS is a cut on the transverse momentum of the online muon, that usually depends slightly on the pseudorapidity of that muon. Due to the deviations between the online and offline reconstruction, a muon trigger is neither fully inefficient for offline muons with a transverse momentum below that threshold, nor fully efficient for muons with a transverse momentum slightly above that threshold; generally speaking, the efficiency of a muon trigger increases steeply in a small interval around the nominal threshold, reaching a plateau in the transverse momentum at the upper edge of that interval. This interval is referred to as the turn-on region of that trigger.

For most applications of trigger efficiencies, in particular for the calculation of event weights, the exact behaviour of the trigger efficiency in the turn-on region is not of interest. The reason for this is the large absolute dependence on the muon transverse momentum in this region, such that the efficiency in the turn-on region is extremely sensitive to the $p_{T}$ distribution in the sample it is measured from. As this distribution differs significantly in the various physics processes that usually contribute to the composition of selected events in an arbitrary physics analysis, the turn-on region is usually not used for the calculation of event weights, and muons with a transverse momentum in this region are not considered for trigger matching. By convention, for the remainder of this section, such muons are not assigned an online match, such that for muons in the turn-on region of any muon trigger the efficiency will be 0 . Although this does not reflect the actual performance of the detector in these regions, it serves the purposes of the presented studies best. 
For the muon triggers that were used in 2011, the efficiencies have been found to reach the plateau for a transverse momentum roughly $2 \mathrm{GeV}$ above the nominal threshold. The minimal $p_{T}$ threshold for an unprescaled single muon trigger was $18 \mathrm{GeV}$. The threshold on the muon $p_{T}$ could be lowered to $10 \mathrm{GeV}$ for triggers requiring two muons, and to $6 \mathrm{GeV}$ for triggers requiring one muon and one electron. Figure 5.2 shows a model for the efficiency of a muon trigger as a function of the muon $p_{T}$.

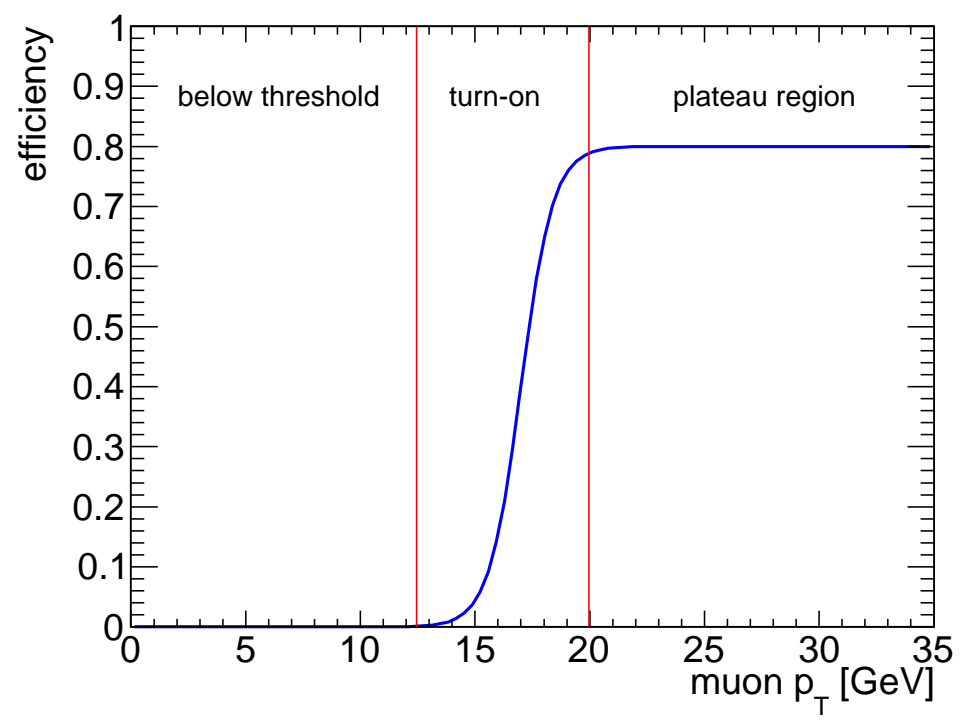

Figure 5.2: A model for the turn-on of a muon trigger with a $p_{T}$ threshold of roughly 18 $\mathrm{GeV}$. Far below the threshold, the efficiency is zero. Due to the limited resolution of the trigger algorithms with respect to the offline algorithms, the efficiency starts to rise a few GeV below the nominal threshold in the turn-on region. A few $\mathrm{GeV}$ above the threshold, the efficiency flattens out as a function of the muon $p_{T}$. This is the plateau region, which is the only region considered in the following. For the ATLAS muon triggers, the efficiency in the plateau region is not necessarily flat, as it depends on other properties of the muon which may be correlated to the transverse momentum of the muon.

\subsubsection{Interpretation of Measurements of Trigger Efficiencies}

For a measurement of a trigger efficiency from data, the numbers $N_{E}$ or $N_{O}$ and $K_{E}$ or $K_{O}$ have to be determined from some data sample. Naturally the true efficiency cannot be measured by evaluating the limit $N_{i} \rightarrow \infty$. Instead an estimator for the true efficiency has to be defined using some measurement $n \in \mathbb{N}$ and $k \in \mathbb{N}$. A naive estimator of the trigger efficiency is given by the fraction

$$
\hat{\varepsilon}=\frac{k}{n} .
$$

Using a more elaborate method aiming for an assessment of the uncertainties on the estimated efficiency, the measurement of the numbers $n$ and $k$ can be interpreted in a Bayesian way in order to calculate an estimator and an uncertainty for the efficiency. Using Bayes' Theorem with 
flat priors, and modelling the trigger decision as a Bernoulli process, the probability distribution function for the efficiency $\varepsilon$ is given by

$$
p(\varepsilon \mid n, k)=\frac{(n+1) !}{k !(n-k) !} \varepsilon^{k} \cdot(1-\varepsilon)^{n-k},
$$

and an estimator for the efficiency is given by

$$
\hat{\varepsilon}=\operatorname{mode}(p(\varepsilon))=\frac{k}{n},
$$

which gives the same value as the naive estimator (5.4). Other prior distributions can as well be used - throughout this chapter however flat priors are considered only and equation (5.5) is used. The uncertainty on the measured efficiency can be calculated from the boundaries of the smallest interval covering $68 \%$ around the mode, i.e.

$$
\int_{\hat{\varepsilon}-\Delta^{\text {low }}}^{\hat{\varepsilon}+\Delta^{\text {high }}} p(\varepsilon) d \varepsilon=0.68 \quad \text { for } \Delta^{\text {high }}+\Delta^{\text {low }}=\text { minimal. }
$$

If $n$ is large enough, and if $\varepsilon$ is sufficiently different from 0 and 1, the distribution $p(\varepsilon)$ becomes roughly Gaussian, where the mean value $\mu$ and the width $\sigma$ are given by

$$
\begin{aligned}
\mu & =\frac{k}{n} \quad \text { and } \\
\sigma^{2} & =\frac{\mu \cdot(1-\mu)}{n} .
\end{aligned}
$$

For most applications, this is a reasonable approximation, as will be detailed later in this chapter.

At this point, it must be emphasised that a measurement of a trigger efficiency is in most cases a biased measurement. As already outlined above, for an object based trigger, the efficiency $\varepsilon$ is usually a function of the object properties $x_{O}$,

$$
\varepsilon=\varepsilon\left(x_{O}\right)
$$

and depending on the geometry and structure of the detector, this function is usually unknown and not analytical. In such a case, the measurement of the efficiency of a trigger is always a binned measurement, and the measured efficiency in one bin is always the average efficiency with respect to the sample $S$ that measurement was performed on. In the case of infinite statistics, the efficiency in some bin $j$, defined by some lower and upper value of each $x_{O}$, is given by

$$
\varepsilon_{j}=\int_{j} \ldots \int_{j} d x_{1} \ldots d x_{I} p_{S}\left(x_{1}, \ldots, x_{I}\right) \varepsilon\left(x_{1}, \ldots, x_{I}\right)=\varepsilon_{j}(S),
$$

where the p.d.f. $p_{S}\left(x_{1}, \ldots, x_{I}\right)$ is given by the relative differential cross-sections with respect to the object properties $x_{O}$, augmented by any other relevant observable in each event. This means that a measurement of the efficiency of the very same trigger, performed on two different samples, may yield two significantly different estimators for the same quantity. It is, however, reasonable to assume that the differences become smaller with an increasing granularity in the measurement, such that if the binning is chosen fine enough, the measurements are nearly unbiased. This assumption will be tested for the ATLAS muon triggers later in this chapter. 


\subsection{Application of Trigger Efficiencies in Data Analyses}

There are different approaches to how the trigger efficiencies as measured from data are used in an analysis. In searches for new physics, for instance, MC simulations are used to generate signal events and compare the expectations from models beyond the SM with the actual observations. In data events are selected only if they are picked up by one of the used triggers, and if the efficiency of the trigger - or combination of triggers - that is used in an analysis differs significantly from 1, this efficiency has to be considered for any data-MC comparison. The ATLAS lepton triggers are not $100 \%$ efficient - in particular the muon triggers can have large inefficiencies.

It is possible to simulate as well the relevant triggers and only use events in the MC that passed the simulated triggers in the very same way as it is done in data with the actual triggers. In order to ensure comparability between $\mathrm{MC}$ and data, the trigger efficiencies in question have to be measured in data as well as in MC and a correction factor has to be applied to the MC usually. A different approach neglects the trigger simulation completely and applies a weight to each MC event, corresponding to the absolute probability that this event was selected by the used trigger items in data. Both approaches are briefly compared here, after which a detailed description of the calculation of event weights for different triggers and the calculation of uncertainties on the events weights and the sum of event weights is given. In the following, only object based triggers are considered.

\subsubsection{Scale Factors and Event Weights}

A widely used approach in ATLAS is the calculation of trigger scale factors (SF) for the comparison of data and MC. In the case of a single object trigger and a single object of interest in the final state, the scale factor is just the ratio of the trigger efficiency in data and the trigger efficiency in $\mathrm{MC}$

$$
s_{1}=\frac{\hat{\varepsilon}_{\text {data }}}{\hat{\varepsilon}_{\mathrm{MC}}}
$$

where the trigger efficiencies $\varepsilon_{i}$ are usually a function of the properties of the objects to be triggered on. However, it is usually assumed that some of the dependencies cancel by building the ratio, such that the scale factors (5.10) depend on less parameters, which means that the efficiencies do not have to be measured as a function of all relevant variables. For this to be true, it is of particular importance that the efficiencies $\varepsilon_{\mathrm{MC}}$ and $\varepsilon_{\text {data }}$ have been measured on a comparable dataset. To ensure this, the efficiency measurements are usually performed using only objects from a well defined physics process in $\mathrm{MC}$ and performing a background subtraction in data, i.e. contributions to the numerator and the denominator in equation (5.6) from different physics processes are estimated and subtracted separately. If the efficiencies are measured as a function of all relevant variables with a fine enough granularity, the requirement of a background subtraction can usually be relaxed. It is in general assumed that the scale factors obtained from a sample covering a well defined physics process can universally be applied to any MC sample no matter what the physics content of that sample is.

If the trigger signature is more complicated and/or if the number of objects of interest in the final state is increased, the calculation of scale factors gets more complicated as well. The most general expression for a scale factor is then given by the ratio

$$
s_{a}=\frac{W\left(\hat{\varepsilon}_{\mathrm{data}}^{i}\right)}{W\left(\hat{\varepsilon}_{\mathrm{MC}}^{i}\right)},
$$


where $W$ is the probability for a given event to satisfy the trigger requirement, which in good approximation is a function of the single object trigger efficiencies $\varepsilon^{i}$. It is important to note that the expression (5.11) can usually not be written as a function of ratios like (5.10) only. Therefore, even if for the single object trigger efficiencies the ratio $s_{1}$ does not depend on all object properties that the efficiencies themselves depend on, this is in general not true for the ratio $s_{a}$, such that for the calculation of these scale factors the trigger efficiency has to be measured precisely as a function of all relevant object properties in both data and MC. This induces the necessity of four independent steps in the application of scale factors, i.e. the simulation of the trigger, measurement of the trigger efficiency in $\mathrm{MC}$, measurement of the trigger efficiency in data and calculation of the probabilities $W$ respectively the ratio of these. Each of these steps introduces sources of uncertainties - in particular the statistical uncertainty from the measurement of the trigger efficiency in the MC as well as the accurate background subtraction, which introduces uncertainties that are correlated to the uncertainty on the total luminosity, are of importance.

Another disadvantage of the trigger scale factors is the limited use of the available MC statistics, in particular in final states with exactly one muon using a single muon trigger. For central muons the trigger efficiency can easily drop below $70 \%$, such that in this case about $30 \%$ of the computing time during the event generation and detector simulation have been spent for events that are neglected in the analysis. This number gets even higher for signatures with medium transverse momenta using di-muon triggers, as it is the case for instance in searches for SUSY with compressed mass spectra. In the above example only $50 \%$ of the MC statistics were used in that case. Apart from that, the trigger that was used in data has to be included in the MC, otherwise the trigger selection cannot be performed in MC. This is not always the case, and the application of scale factors gets difficult if the performance of the used triggers is significantly different in MC than in data, as it was the case for the combined ATLAS muon triggers during a period in 2011 .

Finally, the correct calculation of uncertainties for scale factors is difficult. Since the trigger efficiencies can only be measured with a finite precision from both data and MC, the scale factors must be assigned a statistical uncertainty as well as a systematic uncertainty. While this is straight forward for a single event, the normalisation of the $\mathrm{MC}$ in one bin of an arbitrary distribution will usually depend on the sum of scale factors for different events. The correlation between the scale factors, and therefore the calculation of the correct uncertainty on a sum of scale factors, is in general very hard to estimate or calculate without the extensive use of pseudo experiments. A widely used approximation is therefore to set the correlation coefficient to 1 between all events, which can overestimate the total uncertainty by a large factor.

A different approach for the consideration of the trigger performance in a physics analysis is to apply the efficiencies as measured from data directly to the MC. The overall idea is to calculate the probability for an event in the MC to issue the triggers that were used during data taking. In data, one or more of the signal objects are required to have issued the trigger, while in MC each signal object is considered in the calculation of the event weight, such that instead of the ratios (5.11) the weight $W_{\text {data }}$ is used directly. In contrast to using the scale factors, this approach makes use off the full MC statistics, as the binary trigger decision in a $\mathrm{MC}$ event is replaced by assigning a weighting factor to it. It is also possible to compare data that was taken using a trigger that is unavailable or flawed in the MC. The calculation is done in only two of the four steps that are needed to calculate the scale factors and the calculation of the uncertainties on the sum of weights can be performed taking into account the covariance between two event weights without using pseudo experiments, if a linear approximation is used. On the other hand, due to equation (5.9), if the efficiencies are not measured with a sufficient resolution in all relevant variables, the calculated weights may be significantly off, such that the 
measurements have to be performed with a high granularity.

In the remainder of this section, the calculation of event weights for several triggers and combinations of triggers is detailed, after which the approximation used to calculate the pairwise covariance for event weights is described. Several sanity checks for both the calculation and applicability of event weights and the calculation of uncertainties are presented.

\subsubsection{Calculation of Event Weights}

The calculation of event weights is done starting from single object trigger efficiencies $\varepsilon_{i}$ and corresponds to the calculation of probabilities, such that the calculus of probabilities can be used to build and simplify generic expressions for event weights. Starting with the simplest possible case, a single object trigger, the calculations for symmetric and asymmetric di-object triggers are described. Eventually also combinations of triggers and multi-object triggers are considered. Only lepton triggers are considered here, as leptons are the only objects this technique has so far been used for in ATLAS.

\section{Single Object Triggers}

For an analysis targeting a model or process with one or more high- $p_{T}$ objects of interest of a single type, a single object trigger designed for that type is generally the best choice for the online selection of events. If the final state contains $N$ such objects of interest, for instance muons or electrons, the probability for an event to pass a single object trigger is given by

$$
W_{s}\left(\varepsilon_{i}\right)=1-\prod_{i=1}^{N}\left(1-\varepsilon_{i}\right),
$$

where $\varepsilon_{i}$ is the probability for the $i^{\text {th }}$ object to pass the trigger hypothesis of the used single object trigger [136].

With the Bayesian interpretation of the measurements of $n$ and $k$ in the calculation of a probability distribution function for the single object trigger efficiency it is straight forward to construct a probability distribution for any function of those efficiencies:

$$
p_{W}(W)=\int p_{\varepsilon_{1}}\left(\varepsilon_{1}\right) \ldots p_{\varepsilon_{N}}\left(\varepsilon_{N}\right) \delta\left(W-W\left(\varepsilon_{i}\right)\right) d \varepsilon_{1} \ldots d \varepsilon_{N},
$$

and the mode and the smallest $68 \%$ interval around that mode could be used as an estimator for the weight and the uncertainty on the weight. This interpretation of the above p.d.f. is quite misleading, in particular if the measurement was done with low statistics, due to the intrinsic asymmetry of the p.d.f.s (5.5). The major problem with this interpretation is that the most probable value from the p.d.f. (5.13) does not correspond to the 'natural' value (5.12) in that case. For this reason, the calculation of both the central value and the uncertainty of an event weight are done in a different way. The calculation is performed using equation (5.12) and a Gaussian error propagation is performed, using the frequentist interpretation of the efficiency measurements with symmetric uncertainties on the central value. While this procedure has several shortcomings, it is shown to perform reasonably well for the triggers used in the analyses employing the trigger re-weighting approach later. This is particularly advantageous if more complicated trigger signatures are used. 


\section{Symmetric Di-Object Triggers}

A symmetric di-object trigger searches for two distinct objects passing the same trigger hypothesis in an event. Such triggers are of particular interest for analyses with final states with two or more objects of interest, targeting processes with medium- $p_{T}$ or even low- $p_{T}$ objects. The thresholds in the trigger hypothesis can usually be relaxed due to the smaller rates at which events with larger object multiplicities occur at; this is in particular true for lepton triggers at the LHC.

In the case of lepton signatures (i.e. electrons and/or muons), it has been shown that for such triggers the efficiency can be factorised in single object trigger efficiencies, if the leptons are separated well enough [137]. As it is reasonable to use only signal leptons for the calculation of the weights in $\mathrm{MC}$ and the trigger requirement in data, and as signal leptons are usually required to be isolated, the calculation of event weights for multi-lepton triggers can be done in a very good approximation using the factorisation of the total weight.

With $N$ objects of interest in an event, the probability for it to be selected by a symmetric di-object trigger is given by one minus the probability that one or zero objects passed the trigger hypothesis, i.e.

$$
W_{s d}=1-\prod_{i=1}^{N}\left(1-\varepsilon_{i}\right)-\sum_{j=1}^{N} \varepsilon_{j} \prod_{i=1, i \neq j}^{N}\left(1-\varepsilon_{i}\right) .
$$

\section{Asymmetric Di-Object Triggers}

Asymmetric di-object triggers select events based on two different trigger hypotheses. In contrast to the symmetric di-object triggers this allows to decrease one of the thresholds, while usually the higher threshold is raised. Such signatures are of importance for physics scenarios where the correlation between two or more objects of interest is not too high, as it is the case in many multi-vertex cascade decays in SUSY scenarios, for instance. Also, while for the symmetric diobject triggers at ATLAS usually both objects must pass the L1, the L2 and the EF hypothesis, asymmetric di-object triggers can be configured to skip the L1 and L2 requirement on the second object. In particular for the ATLAS muon triggers, where the major contribution to the overall inefficiency arises at L1 already, this relaxation can lead to a significant increase in the total efficiency of an asymmetric di-object trigger.

An event passes an asymmetric di-object trigger if at least one object of interest in the event passes the trigger hypothesis with the higher threshold (in the following referred to as the first leg of the trigger) and if in addition a different object of interest passes the trigger hypothesis with the lower threshold (in the following referred to as the second leg of the trigger). The calculation for event weights for asymmetric di-object triggers as outlined here corresponds to the case that both hypotheses require the same type of object, i.e. both objects of interest must be muons, or both objects of interest must be electrons, for instance. Other cases are considered later.

The calculation of weights for asymmetric di-object triggers is more complicated in comparison to the calculation of weights for symmetric di-object triggers because the trigger hypotheses are distinct, and a larger set of efficiencies is needed. An event with two or more objects of interest does not pass an asymmetric di-object trigger

(a) if no object of interest passes the first leg of the trigger, or

(b) if no object passes the second leg of the trigger under the condition that at least one object passes the first leg of the trigger, or 
(c) if both legs of the trigger were passed by and only by the same object of interest.

For the calculation of the probabilities for each of these cases, four different trigger efficiencies have to be measured. These are:

- $\varepsilon_{i}^{1}$ : The absolute probability for the $i^{\text {th }}$ object of interest to pass the first leg of the trigger.

- $\varepsilon_{i}^{2}$ : The absolute probability for the $i^{\text {th }}$ object of interest to pass the second leg of the trigger.

- $\varepsilon_{i}^{2^{\prime}}$ : The probability that the $i^{\text {th }}$ object of interest passes the second leg under the condition that it did not pass the first leg.

- $\varepsilon_{i}^{2 *}$ : The probability that the $i^{t h}$ object of interest passes the second leg under the condition that it did pass the first leg.

These efficiencies are not independent of each other, but the equation

$$
\varepsilon_{i}^{2}=\varepsilon_{i}^{2^{\prime}} \cdot\left(1-\varepsilon_{i}^{1}\right)+\varepsilon_{i}^{2 *} \cdot \varepsilon_{i}^{1}
$$

must hold. The probability for no object of interest to pass the first leg of the trigger - case (a) - in an event with $N$ objects of interest is given by

$$
p_{a}=\prod_{i=1}^{N}\left(1-\varepsilon_{i}^{1}\right) .
$$

The term for case (b) is calculated using conditional trigger efficiencies for the second leg. The term that enters the calculation of the event weight is given by $p\left(\overline{2^{\text {nd }} \operatorname{leg}} \mid 1^{s t} \operatorname{leg}\right) \cdot p\left(1^{s t} \operatorname{leg}\right)$. The measurement of the needed efficiencies as well as the factorisation of this term is much easier by using the sum rule for conditional probabilities for a binary condition. With arbitrary events $A$ and $B$, the equation

$$
p(\bar{A} \mid B) \cdot p(B)=p(\bar{A})-p(\bar{A} \mid \bar{B}) \cdot p(\bar{B})
$$

must also hold for the case (b). The corresponding term in the calculation of the weight is thus given by

$$
p_{b}=\prod_{i=1}^{N}\left(1-\varepsilon_{i}^{2}\right)-\prod_{i=1}^{N}\left(1-\varepsilon_{i}^{2^{\prime}}\right) \prod_{i=1}^{N}\left(1-\varepsilon_{i}^{1}\right) .
$$

Finally, the probability for the case (c) is given by

$$
p_{c}=\sum_{i=1}^{N} \varepsilon_{i}^{1} \varepsilon_{i}^{2 *} \cdot \prod_{j=1, j \neq i}^{N}\left(1-\varepsilon_{j}^{1}\right) \cdot \prod_{j=1, j \neq i}^{N}\left(1-\varepsilon_{j}^{2^{\prime}}\right) .
$$

These terms are combined to the total weight for an asymmetric di-object trigger, which is given by

$$
W_{a d}=1-p_{a}-p_{b}-p_{c}
$$




\section{Combinations of Triggers}

There are different types of trigger combinations that can be used to increase the absolute selection efficiency. In terms of lepton triggers, the simplest combination is a logical OR of a single electron trigger and a single muon trigger, which could for instance be used to select events with top-pairs where both top quarks decay leptonically, or for instance SUSY searches for direct chargino production. Assuming again that the single object trigger efficiencies are independent of each other, the weight to be applied in MC if an OR of a single muon and a single electron trigger is used in data for an event with $N_{\mu}$ signal muons and $N_{e}$ signal electrons is given by

$$
W_{e \| \mu}=1-\prod_{i=1}^{N_{\mu}}\left(1-\varepsilon_{i}^{\mu}\right) \prod_{j=1}^{N_{e}}\left(1-\varepsilon_{j}^{e}\right) .
$$

It is also possible to use triggers that require the existence of both an electron and a muon in the event. These are of use in the same scenarios as the OR of the single muon and single electron trigger, but usually the thresholds on the objects can be significantly decreased if such a multiobject trigger is used. The calculation of the event weights in that case is quite straight forward.

As for the asymmetric di-object trigger itself, the computation of event weights gets more complicated if two triggers sensitive to the same signature are combined. This is for instance the case if a symmetric di-muon trigger is ORed with a single muon trigger. In this case the easiest way to calculate an event weight is to make use of conditional efficiencies for the symmetric di-object trigger. Using

- $\varepsilon_{i}^{s}$, the absolute efficiency for the $i^{\text {th }}$ object of interest of the single object trigger, and

- $\varepsilon_{i}^{d^{\prime}}$, the probability for the $i^{\text {th }}$ object of interest to pass the hypothesis of the symmetric di-object trigger under the condition that it does not pass the hypothesis of the single object trigger,

the probability to pass either the single object trigger or the symmetric di-object trigger is given by:

$$
p(s \| s d)=p(s)+p(s d \mid \bar{s}) \cdot p(\bar{s})
$$

with $p(s)$ given by equation (5.12) inserting the efficiencies $\varepsilon_{i}^{s}$ and $p(s d \mid \bar{s})$ given by equation (5.14) using the efficiencies $\varepsilon_{i}^{d^{\prime}}$.

\subsubsection{Calculation and Propagation of Uncertainties on Event Weights}

The measurement of the trigger efficiency from data can only be performed with finite statistics, which means that the efficiencies will always come with a statistical uncertainty. Apart from that, the efficiencies can only be measured with a finite resolution in terms of the relevant variables, for instance $\eta, \phi$ and $p_{T}$ for the muons. This means that the efficiencies provided as an input for the calculation of event weights will be binned measurements, and within one bin the efficiency will not always be flat. In particular for the ATLAS muon triggers the efficiency as a function of the muon $\eta$ is highly inhomogeneous in the barrel part of the detector. Even if a fine binning is used, this may cause differences in the average efficiency per bin if samples with a different physics content are compared, cf. section 5.2.3. 
The finer the binning for the efficiency measurements is chosen, the smaller these systematic shifts get, but the statistics per bin decrease, which means that the statistical uncertainty in each bin increases. The binning must therefore be chosen in a reasonable way, countervailing the statistical uncertainty with the systematic uncertainty due to the different distributions in the relevant observables. The estimation of the systematic uncertainty will be assessed later in this chapter. In this section, the treatment of the statistical uncertainty, in particular the uncertainty on the sum of event weights is described.

If event weights, such as trigger weights, are used to adjust MC predictions to data, it is usually the sum of the event weights in the MC that is compared to the event count in data. Most generally, for a total of $N_{e}$ raw MC events, that sum is given by the expression

$$
S=\sum_{n=1}^{N_{e}} W_{n}
$$

where $W_{n}$ is the weight of the $n^{\text {th }}$ event. Each of these weights is usually assigned an uncertainty, such that

$$
W_{n}=W_{n}^{+\sigma_{W n}^{\text {down }}}
$$

and a widely used procedure to estimate the uncertainties on $S$ is to simultaneously vary each weight up and down by one standard deviation, such that

$$
S=S_{-\sigma_{S}^{\text {down }}}^{+\sigma_{\text {up }}^{\text {up }}} \quad \text { with } \sigma_{S}^{\text {up } / \text { down }}=\sum_{n=1}^{N_{e}} \sigma_{W_{n}}^{\text {up } / \text { down }} .
$$

This procedure will in most cases overestimate the uncertainties on $S$, as it assumes the correlation coefficients for each pair of event weights to be one.

As described above, for the calculation of trigger weights the efficiencies are provided as a number of independent measurements, there is one measurement per bin that is little correlated to the measurements in any other bin. Therefore the covariance of two of these measurements in some bins $i$ and $j$ can be assumed to be

$$
\operatorname{cov}\left(\varepsilon_{i}, \varepsilon_{j}\right)=\delta_{i}^{j} \sigma_{i} \sigma_{j}
$$

Let $W_{n}$ and $W_{m}$ be two event weights that are calculated using some set of efficiencies $\varepsilon_{i}$, such that

$$
W_{n}=W_{n}\left(\varepsilon_{i}\right) \quad \text { and } \quad W_{m}=W_{m}\left(\varepsilon_{i}\right) .
$$

The most general expression for the covariance of these weights using a Bayesian interpretation of the efficiency measurements is given by

$$
\begin{aligned}
\operatorname{cov}\left(W_{n}, W_{m}\right) & =E\left[\left(W_{n}-E\left[W_{n}\right]\right) \cdot\left(W_{m}-E\left[W_{m}\right]\right)\right] \\
& =\int \ldots \int\left(W_{n}-E\left[W_{n}\right]\right) \cdot\left(W_{m}-E\left[W_{m}\right]\right) p\left(\varepsilon_{1}\right) \ldots p\left(\varepsilon_{I}\right) d \varepsilon_{1} \cdots d \varepsilon_{I}
\end{aligned}
$$

where $E[x]$ is the expectation value of $x$ and equation (5.26) has been used. If two conditions are satisfied, this expression can be simplified. The first condition concerns the p.d.f.s for the efficiencies, which must be Gaussian, such that

$$
\hat{\varepsilon_{i}}=\frac{k}{n}=E\left[\varepsilon_{i}\right] \quad \text { and } \quad \sigma_{\varepsilon_{i}^{\text {up }}}=\sigma_{\varepsilon_{i}^{\text {down }}} .
$$


As outlined above, this is in general not true for efficiency measurements. It is however a reasonable approximation if the efficiencies are sufficiently different from 0 and 1 and if the statistics that are available for the measurements are not too small. For muon triggers, this is usually the case; for electron triggers the approximation is worse, but it is still feasible to use it.

The second condition that must be satisfied is that each event weight is a linear function of each efficiency $\varepsilon_{i}$ only, such that

$$
\frac{\partial^{l} W_{n}}{\partial \varepsilon_{i}^{l}}=0 \quad \forall i, \quad \forall l \geq 2 .
$$

Since trigger efficiencies are mostly parametrised as functions of the three-momentum - or some equivalent vector - and as signal leptons are usually required to be separated well enough from each other, this second condition is in general well satisfied for lepton trigger event weights. Equation (5.29) in combination with equation (5.30) implies that

$$
\begin{aligned}
E\left[W_{n}\left(\varepsilon_{1}, \ldots, \varepsilon_{I}\right]=W_{n}\left(E\left[\varepsilon_{1}\right], \ldots, E\left[\varepsilon_{I}\right)\right]\right) & =W_{n}\left(\hat{\varepsilon_{1}}, \ldots, \hat{\varepsilon_{I}}\right) \\
& =\hat{W}_{n}
\end{aligned}
$$

The function $W_{n}\left(\varepsilon_{i}\right)$ can be expanded in a Taylor series at $\varepsilon_{i}=\hat{\varepsilon_{i}}$; using equation (5.31) and omitting terms of cubic order or higher, this yields

$$
W_{n}\left(\varepsilon_{i}\right)=\hat{W}_{n}+\left.\sum_{i=1}^{I} \frac{\partial W_{n}}{\partial \varepsilon_{i}}\right|_{\varepsilon_{k}=\hat{\varepsilon_{k}}}\left(\varepsilon_{i}-\hat{\varepsilon_{i}}\right)+\left.\frac{1}{2} \sum_{i=1}^{I} \sum_{j=1}^{I} \frac{\partial^{2} W_{n}}{\partial \varepsilon_{i} \partial \varepsilon_{j}}\right|_{\varepsilon_{k}=\hat{\varepsilon}_{k}}\left(\varepsilon_{i}-\hat{\varepsilon_{i}}\right)\left(\varepsilon_{j}-\hat{\varepsilon_{j}}\right) .
$$

Due to equation (5.30) mixed terms that are of quadratic order in $\left(\varepsilon_{i}-\hat{\varepsilon_{i}}\right)$ for any $i$ vanish. Also, when equations (5.31) and (5.32) are inserted into equation (5.28) and the integration is carried out, all terms that for some $i$ are proportional to

$$
\int d \varepsilon_{i} p\left(\varepsilon_{i}\right)\left(\varepsilon_{i}-\hat{\varepsilon}_{i}\right)^{2 n+1}=0 \quad n \in \mathbb{N}
$$

vanish because all uneven central moments of a symmetric p.d.f. are equal to zero. Therefore all non-vanishing terms that are omitted in the Taylor expansion (5.32) are of quartic order in the uncertainties of the efficiencies and thereby very small compared to the leading terms. In addition, such terms will only contribute to the correlation factor between two event weights $W_{n}$ and $W_{m}$, if both weights depend on exactly the same subset of efficiencies $\varepsilon_{i}$. In that case, the approximation for the covariance that is derived in the following will underestimate the correlation if in addition the weights depend on more than one efficiency - however the fraction of pairs of events where all relevant properties are nearly equal is small, such that the approximation is expected to yield reasonable results. This hypothesis is tested later in this chapter.

Neglecting - for the above reasons - all terms of quadratic or higher order in the Taylor expansion, the covariance of the event weights $W_{n}$ and $W_{m}$ becomes

$$
\begin{aligned}
& \operatorname{cov}\left(W_{n}, W_{m}\right)= \\
& \quad \int \ldots \int\left(\left.\sum_{i=1}^{I} \frac{\partial W_{n}}{\partial \varepsilon_{i}}\right|_{\varepsilon_{k}=\hat{\varepsilon_{k}}} \cdot\left(\varepsilon_{i}-\hat{\varepsilon_{i}}\right)\right) \cdot\left(\left.\sum_{j=1}^{I} \frac{\partial W_{m}}{\partial \varepsilon_{j}}\right|_{\varepsilon_{k}=\hat{\varepsilon_{k}}} \cdot\left(\varepsilon_{j}-\hat{\varepsilon_{j}}\right)\right) p\left(\varepsilon_{1}\right) \ldots p\left(\varepsilon_{I}\right) d \varepsilon_{1} \ldots \varepsilon_{I},
\end{aligned}
$$


where again due to equation (5.33) all terms with $i \neq j$ vanish, such that the final linear approximation for the covariance of two event weights $W_{n}$ and $W_{m}$ is given by

$$
\begin{aligned}
\operatorname{cov}\left(W_{n}, W_{m}\right) & =\left.\left.\sum_{i=1}^{I} \frac{\partial W_{n}}{\partial \varepsilon_{i}}\right|_{\varepsilon_{k}=\hat{\varepsilon}_{k}} \frac{\partial W_{m}}{\partial \varepsilon_{i}}\right|_{\varepsilon_{k}=\hat{\varepsilon}_{k}} \int \ldots \int\left(\varepsilon_{i}-\hat{\varepsilon_{i}}\right)^{2} p\left(\varepsilon_{1}\right) \ldots p\left(\varepsilon_{I}\right) d \varepsilon_{i} \ldots d \varepsilon_{I} \\
& =\left.\left.\sum_{i=1}^{I} \frac{\partial W_{n}}{\partial \varepsilon_{i}}\right|_{\varepsilon_{k}=\hat{\varepsilon}_{k}} \frac{\partial W_{m}}{\partial \varepsilon_{i}}\right|_{\varepsilon_{k}=\hat{\varepsilon}_{k}} \sigma_{\varepsilon_{i}}^{2}
\end{aligned}
$$

This is a linear approximation that is only valid if the above mentioned criteria (5.29) and (5.30) are satisfied in good approximation. The quality of this approximation will also be assessed later in this chapter.

Equation (5.34) has the consequence that technically it can be taken into account in a very simple way. If the efficiencies are provided as histograms in bins $i$, each weight can be calculated from a number of efficiencies $\varepsilon_{i}$. For the calculation of the uncertainty on the sum of weights, another histogram with the same binning is created, and for each weight that is considered in the sum, the additional histogram is filled with one entry for each efficiency that weight depends on. For $W=W\left(\varepsilon_{i}\right)$, the derivative of the weight with respect to each of the efficiencies is calculated, and the value

$$
\left.\frac{\partial W_{n}}{\partial \varepsilon_{i}}\right|_{\varepsilon_{k}=\hat{\varepsilon}_{k}} \cdot \sigma_{\varepsilon_{i}}
$$

is linearly added to the content in bin $i$ for each of the weights. The variance on the sum $S$ of $N_{e}$ weights is then calculated by quadratically adding all bins in the additional histogram, i.e.

$$
\begin{aligned}
\sigma_{S}^{2} & =\sum_{i=1}^{I}\left(\left.\sum_{n=1}^{N_{e}} \frac{\partial W_{n}}{\partial \varepsilon_{i}}\right|_{\varepsilon_{k}=\hat{\varepsilon_{k}}} \cdot \sigma_{\varepsilon_{i}}\right)^{2} \\
\stackrel{(5.34)}{=} & \sum_{n=1}^{N_{e}} \sum_{m=1}^{N_{e}} \operatorname{cov}\left(W_{n}, W_{m}\right),
\end{aligned}
$$

if the uncertainties $\sigma_{\varepsilon_{i}}$ are propagated to the weights $W_{n}$ using Gaussian propagation of uncertainties.

\subsubsection{Sanity Checks for Trigger Reweighting}

In this section a closure test for the calculation of trigger weights for different physics samples is presented. The goal of the presented study was to assess the size of the systematic shifts in the calculated sum of weights for some physics sample $S^{\prime}$ if the efficiencies that are used for this calculation have been measured from a different sample $S$. As it is a realistic scenario, $S$ in this case is a $Z \rightarrow \mu \mu$ sample. In addition, the quality of the approximation (5.34) is tested.

\section{Closure Test for Event Weights}

As a first step of the closure test, the efficiencies of the simulated muon triggers have been measured from a $Z \rightarrow \mu \mu \mathrm{MC}$ sample using the Tag and Probe method, cf. 5.4.1. Assuming that there is no large dependence on other variables, the muon transverse momentum, the muon azimuthal angle and the muon pseudorapidity have been used as parameters for the efficiencies, such that here $x_{O}=\left(p_{T}, \eta, \phi\right)$. By comparing differently coarse one dimensional measurements 
in each of the three variables, a test binning is defined which is then used to calculate event weights for other MC samples with a different physics content. In a final step, for each sample the sum of the event weights is compared to the event yields that are obtained by using the trigger simulation - the configuration of the trigger simulation is (nearly) the same in each of the compared samples. The closure test has been performed using the simulation for the trigger EF_mu18, which was one of the primary muon triggers for data taking in 2011, and all used samples are part of the official ATLAS $m c 10 b$ production.

The measurement of the trigger efficiencies from the $Z \rightarrow \mu \mu$ sample is shown in Figure 5.3. In each plot, the blue circles show the efficiency in a very fine binning where all other dimensions are integrated out. For the calculation of the weights the efficiencies are considered as a function of all three variables, and each dimension is divided into bins according to the red triangles. A much finer binning is not feasible due to the limited statistics in each bin. With the chosen binning, a total of roughly 1.6 million probe muons is distributed in 6534 bins, such that the statistics in each bin is sufficient.

Figures 5.3(a) and 5.3(b) show the efficiency of EF_mu18 as a function of the muon transverse momentum for the barrel and the endcaps separately. The trigger reaches its plateau efficiency in both regions for $p_{T}>20 \mathrm{GeV}$, as can best be seen from the coarse binning. Above that threshold an efficiency of roughly $70 \%(85 \%)$ is reached in the barrel (endcaps). Figures 5.3(c) and 5.3(d) show the efficiency as a function of the muon azimuthal angle for $p_{T}>20 \mathrm{GeV}$. It can be seen from these plots that the efficiency of the EF_mu18 trigger depends heavily on the azimuthal angle and is not the same for all muons with $p_{T}>20 \mathrm{GeV}$. Finally, Figure 5.3(e) shows the efficiency as a function of the muon pseudorapidity for all muons with $p_{T}>20 \mathrm{GeV}$, which is highly inhomogeneous in the central region.

While it is impossible to define a binning that describes all features of the dependence of the trigger efficiency on all variables equally well, the chosen binning is expected to reproduce the performance of the trigger simulation reasonably well. It is in general difficult to gain enough statistics for a fine binning in the transverse momentum for $p_{T}>45 \mathrm{GeV}$, in particular with the used $Z \rightarrow \mu \mu$ sample. As the performance of the triggers is not expected to change rapidly with the transverse momentum in that region one large bin in $p_{T}$ was chosen to cover all muons with $p_{T}>50 \mathrm{GeV}$. The oscillating structure in the muon azimuthal angle is also difficult to cover completely, such that a relatively coarse binning had been chosen here. Finally, the inhomogeneity in the muon pseudorapidity requires a much finer binning in the barrel region than in the endcaps in this variable.

As a first test, a simple analysis selecting events with exactly one reconstructed muon and at least one primary vertex with more than 4 tracks associated to that vertex has been run on a $W \rightarrow \mu \nu$ sample, where the systematic shifts in the average trigger efficiencies are expected to be relatively small. From the full $W \rightarrow \mu \nu$ sample a total of 20 uncorrelated subsamples with 50, 000 events each have been drawn, and the simple analysis has been run twice on each of the samples. In the first run, the trigger simulation was used to select only such events where the selected signal muon passes the hypothesis of the EF_mu18 trigger. In a second run, the trigger requirement has been removed and an event weight according to the efficiencies as shown in Figure 5.3 has been calculated. The sum of weights has then been compared to the yield from the first run, in total as well as for several distributions.

The uncertainty on the sum of event weights is calculated using the procedure described above, with one exception: As in this case exactly one muon is required in the final state, the trigger weight for an event is just given by the absolute efficiency of the trigger EF_mu18 for the muon in that event. As described above, for more complicated weights the uncertainties on the trigger efficiencies are assumed to be Gaussian and symmetric. The case of a single muon in 


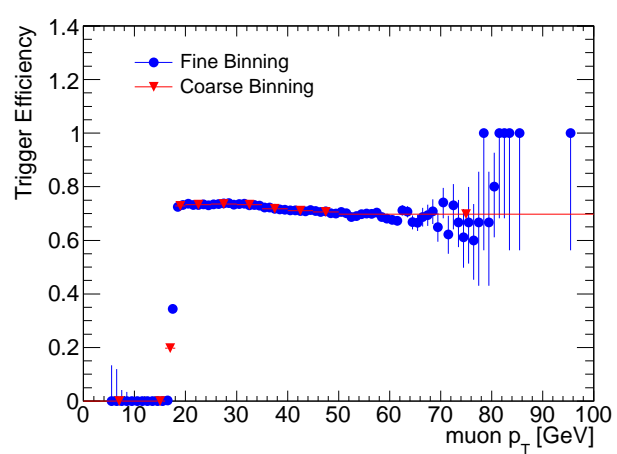

(a) barrel

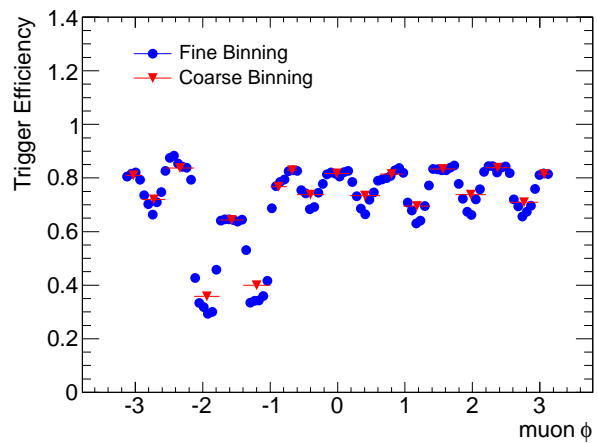

(c) barrel, $p_{T}>20 \mathrm{GeV}$

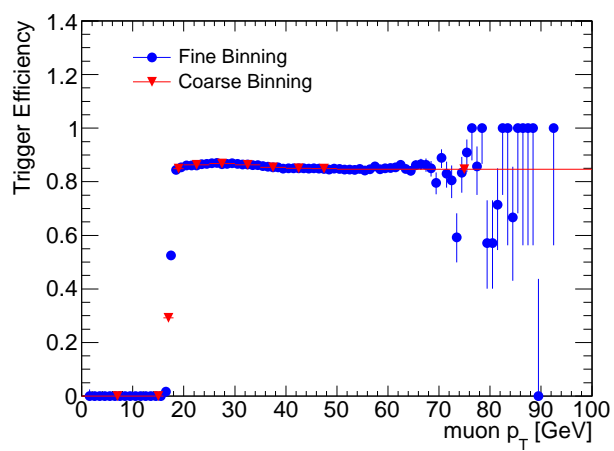

(b) endcaps

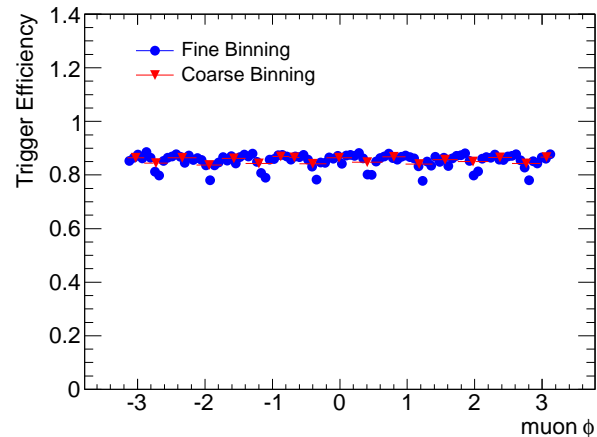

(d) endcaps, $p_{T}>20 \mathrm{GeV}$

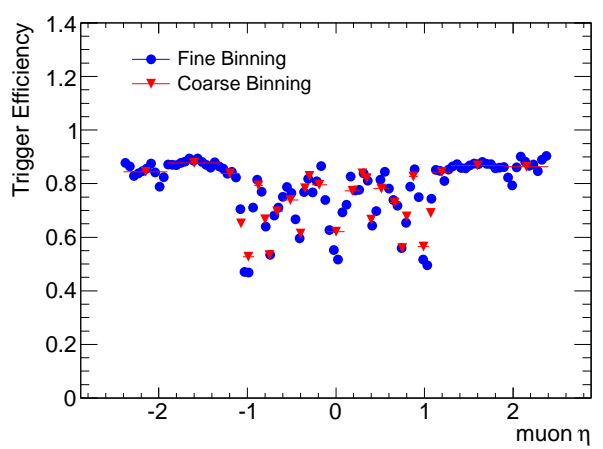

(e) $p_{T}>20 \mathrm{GeV}$

Figure 5.3: The efficiency of the simulated trigger item EF_mu18 in $m c 10 b$ in a $Z \rightarrow \mu \mu$ sample as a function of the muon $p_{T}$ in the barrel (a) and the endcaps (b), as a function of the muon $\phi$ for $p_{T}>20 \mathrm{GeV}$ in the barrel (c) and the endcaps (d), and as a function of the muon $\eta$ for $p_{T}>20 \mathrm{GeV}$. In all plots the efficiency is shown in the binning that was used for the closure test presented in this chapter (red triangles) and in a much finer binning to illustrate the dependence on some variables in some parts of the detector (blue circles).

the final state can be used to justify that choice by using the asymmetric uncertainties on the trigger efficiencies and showing that this results in roughly symmetric uncertainties on the sum of weights; the procedure used here is not fully correct, as the uncertainties are still assumed to 
be Gaussian, but a two-sided Gaussian with different widths is used and the absolute value of the upper and lower uncertainty on the sum of weights are compared.

Assuming that the sum of weights divided by the total number of events passing all cuts but the trigger cut corresponds to the actual average efficiency (in total, or in a bin for the comparison of binned distributions) an uncertainty on the expectation value (which is the sum of weights) is also estimated from the underlying binomial distribution. This uncertainty is estimated using

$$
(\Delta N)^{2}=N_{\text {pass }} \cdot\left(\frac{S}{N_{\text {pass }}}-\frac{S^{2}}{N_{\text {pass }}^{2}}\right)
$$

where $N_{\text {pass }}$ is the number of events passing all cuts in the simple analysis apart from the trigger cuts and $S$ is the sum of the trigger weights. For the comparison of the event yields with the sums of the trigger weights, these uncertainties are squared and the total uncertainty

$$
\sigma_{\text {Rew }}^{2}=(\Delta N)^{2}+\sigma_{S}^{2},
$$

is calculated, where in the case of asymmetric uncertainties $\sigma_{S}=\sigma_{S}^{\text {up }}$ if $N_{\text {trig }}>S$ and $\sigma_{S}=\sigma_{S}^{\text {low }}$ if $N_{\text {trig }}<S$. Since the used samples are fully correlated for both runs before the trigger cut is applied in the first run, no further uncertainty needs to be considered for this comparison. For a comparison between data and re-weighted MC, the term $(\Delta N)^{2}$ is included in the width of the Poissonian distribution that is considered in that case.

The result of the test is shown in Figure 5.4. The absolute numbers are also listed in Table 5.1. Figure 5.4(a) compares the event yield in the run using the trigger simulation with the sum of weights obtained in the run using the trigger re-weighting as a function of the sample number. The corresponding pull distribution is shown in Figure 5.4(b); although the uncertainties are not fully Gaussian, the pull is reasonably well described by a Gaussian function. The fitted mean value is in good agreement with 0 , which means that the binning and the trigger efficiencies that have been used to re-weight the sample do not introduce a large bias. Also, the fitted variance of the Gaussian distribution is in good agreement with 1 showing that the equation (5.37) is a good estimator for the uncertainties.

For some physics analyses, it is not only important to get the absolute event yields right, but typically several distributions are of interest. For the closure test on the $W \rightarrow \mu \nu$ sample, for each sample the distributions of the muon transverse momentum, the muon azimuthal angle and the muon pseudorapidity have been examined, since these are the properties of the muons on which the trigger efficiency shows the strongest dependence and systematic shifts are expected to be most prominent in these distributions. In addition, the distribution of the missing transverse energy has been compared between the two test runs. The results for the sample \#13 are shown in Figure 5.5.

The distributions for the two runs in sample \#13 show an overall excellent agreement. There are bins in which the deviations are larger than $3 \sigma$ indicating that systematic shifts at the order of the statistical uncertainty (5.38) are introduced by the re-weighting procedure. With the used binning for the trigger efficiencies, this effect is expected to become more significant if the binning in the distributions is increased, as it is shown as an example in Figure 5.6.

To further assess the size of the systematic shifts, for each distribution and each bin a pull distribution has been created using the 20 samples. While 20 ensembles is not a large number for this test, the size of the systematic shifts can still be estimated from this study. A normal distribution has been fitted to each of the pulls, as shown for one bin in the muon $\eta$ and one bin in the muon $\phi$ as an example in Figure 5.7(a) and 5.7(b). The fitted mean values and the 


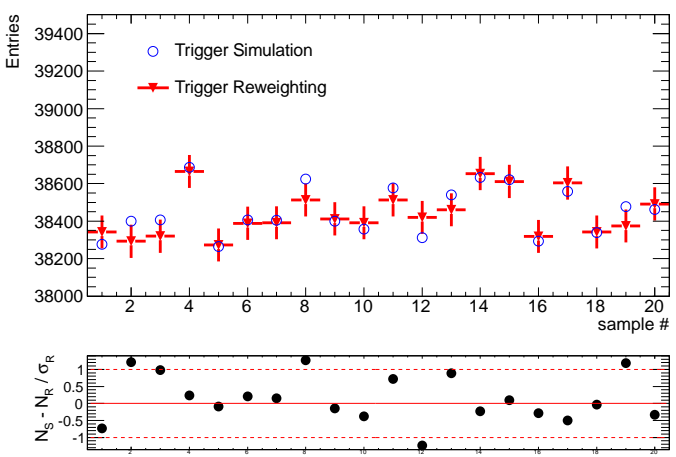

(a)

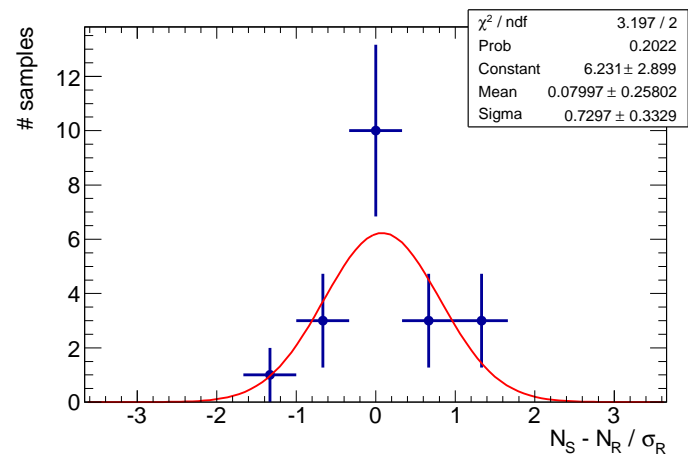

(b)

Figure 5.4: Outcome of the closure test performed on the $W \rightarrow \mu \nu$ sample. On the left side the event yield using the trigger simulation is compared to the sum of weights obtained by applying the trigger re-weighting procedure as a function of the test sample number. The plot on the right side shows the corresponding pull distribution, to which a Gaussian distribution function has been fitted. The fitted mean value is comparable with 0 and the fitted width is comparable with 1 indicating that both the estimator for the sum of weights as well as the estimated uncertainties are unbiased, here.

fitted widths of the normal distributions are shown as a function of the muon $p_{T}$, the muon $\eta$, the muon $\phi$ and the missing transverse energy in Figure 5.7(c) to 5.7(f) for the same binning as used in Figure 5.5. The fitted mean values are mostly comparable to 0, in particular in the muon $p_{T}$ and the $E_{T}$-distributions. As expected the largest differences arise in the muon $\eta$ distributions, where the trigger efficiency is not a very smooth function of the variable. For all distributions, the fitted width is roughly comparable with 1 , such that the estimation of the statistical uncertainty can be regarded to be valid. The systematic shifts in some bins are typically at the order of 1 to 3 times the statistical uncertainty, as expected. 


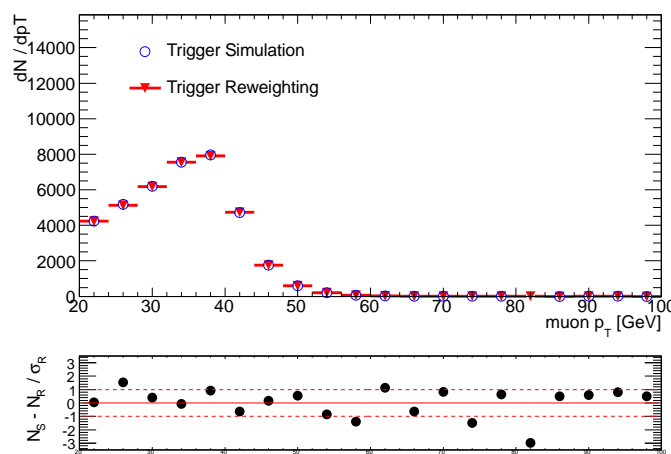

(a)

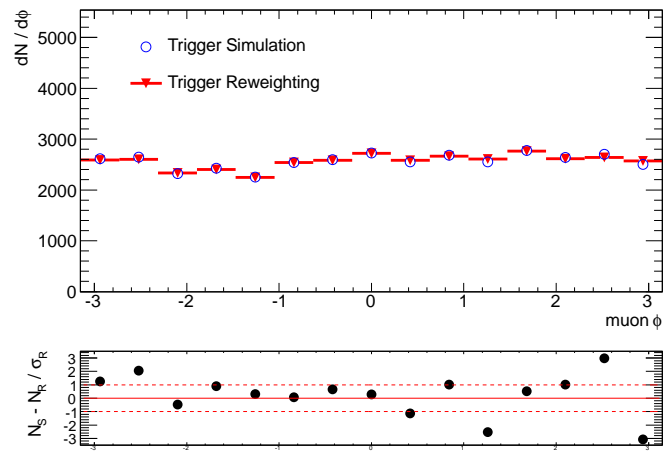

(c)

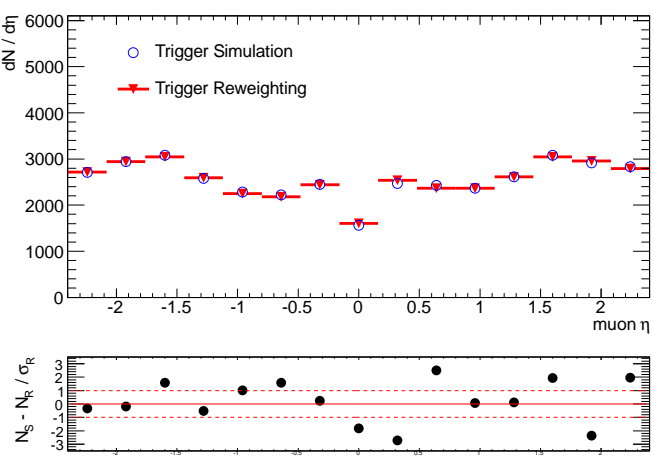

(b)

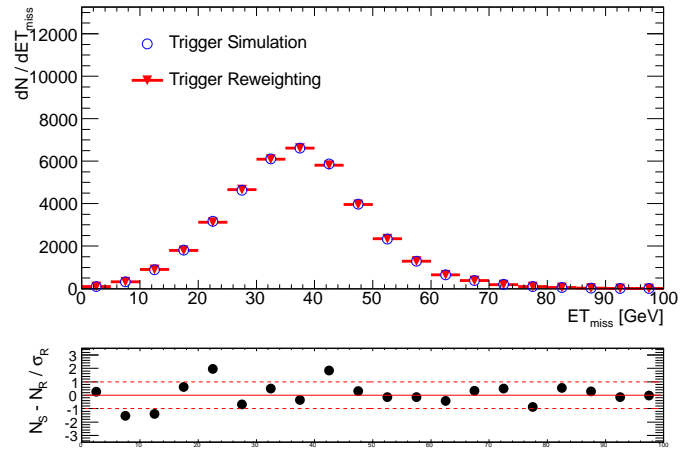

(d)

Figure 5.5: A more detailed comparison between the event yield using the trigger simulation and the sum of weights obtained from the trigger re-weighting for the sample \#13. The distributions of the muon transverse momentum (a), the muon pseudorapidity (b), the muon azimuthal angle (c) and the missing transverse energy (d). The overall agreement is good for all distributions.

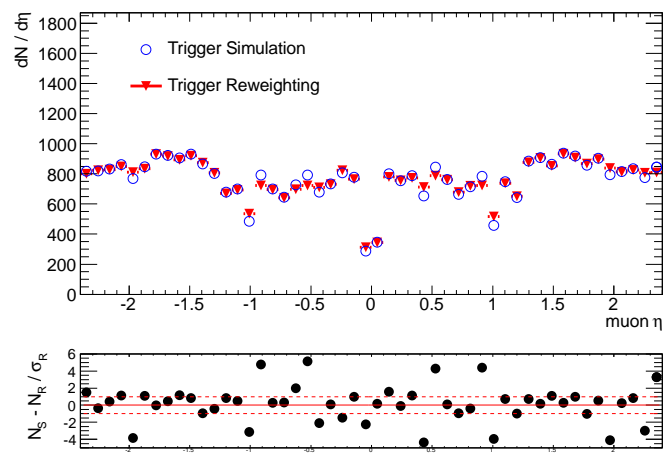

(a)
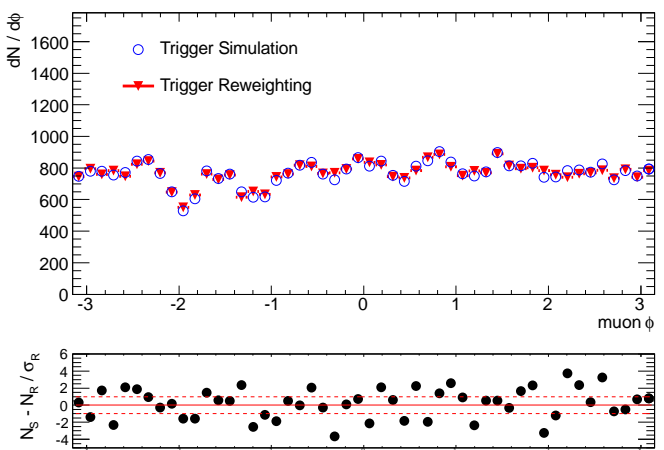

(b)

Figure 5.6: The $\eta$ and $\phi$ distributions from sample \#2 in a much finer binning. While for most bins the agreement is good as for the coarser binning, there are some bins in which systematic shifts are clearly visible. The size of these shifts is of the same size as the combined statistical uncertainty, roughly. 


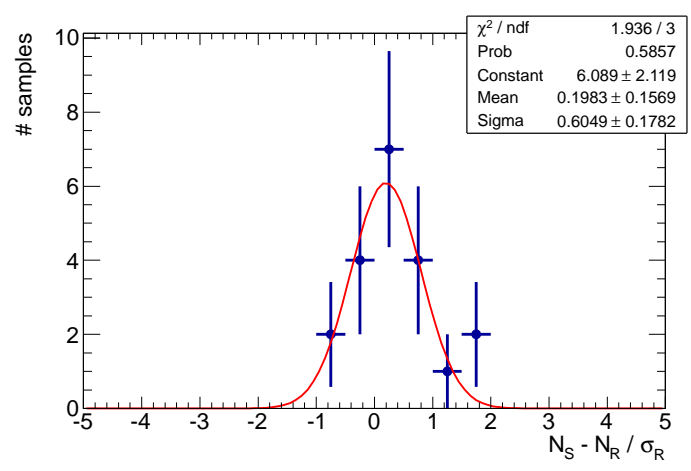

(a) pull distribution for $1.44<\eta<1.76$

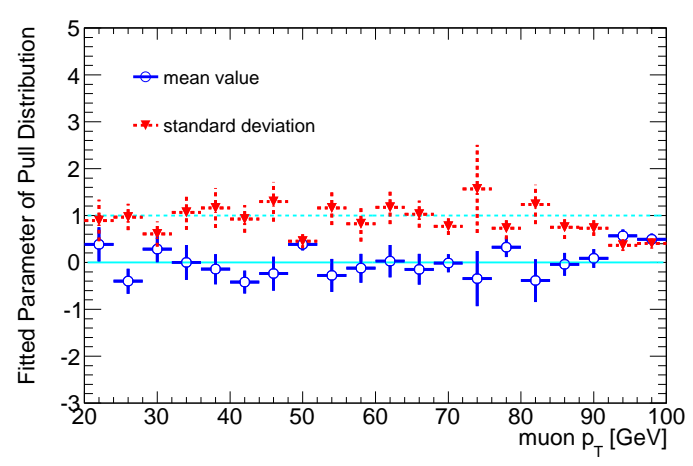

(c) mean and width for $p_{T}$ distribution

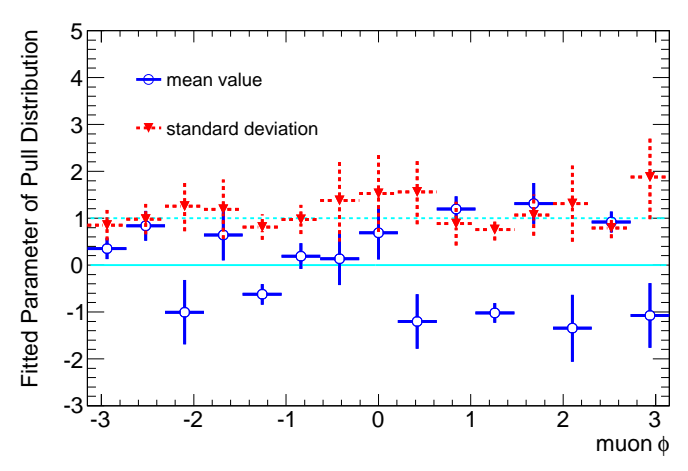

(e) mean and width for $\phi$ distribution

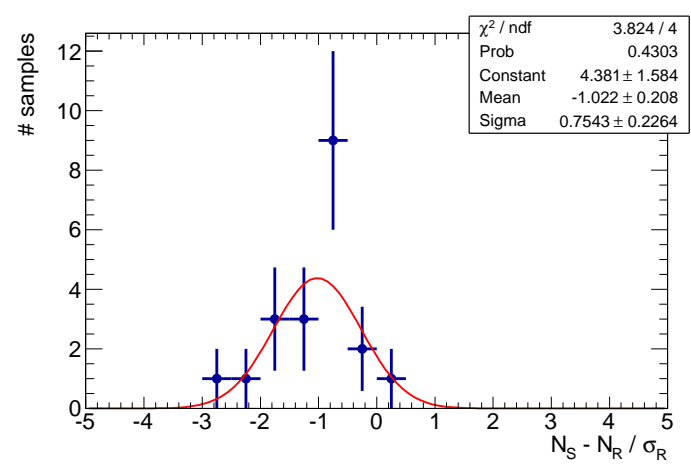

(b) pull distribution for $1.05<\phi<1.47$

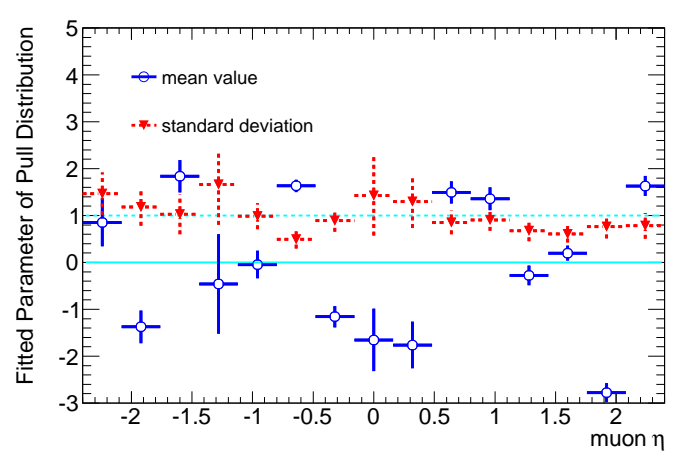

(d) mean and width for $\eta$ distribution

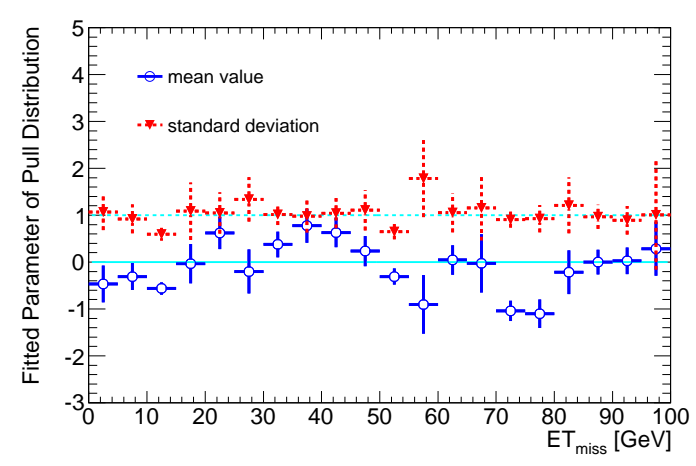

(f) mean and width for $E_{T}$ distribution

Figure 5.7: Complete summary of the closure test on the $W \rightarrow \mu \nu$ for the distributions of the muon $p_{T}$, muon $\eta$, muon $\phi$ and $E_{T}$. In (a) and (b) two example pull distributions using all 20 samples are shown, in (a) for one bin in $\eta$, in (b) for one bin in $\phi$. A Gaussian distribution has been fitted to the pulls, which have been created for all bins in each of the four relevant distributions here. The fitted mean value (blue circles) and the fitted width (red triangles) of the Gaussian distribution are shown as a function of (a) the muon $p_{T}$, (b) the muon $\eta$, (c) the muon $\phi$ and (d) the $E_{T}$. The fitted mean value in some bins differs significantly from 0 , in particular in the muon $\eta$ and the muon $\phi$ distributions, indicating a systematic shift in these bins. The fitted standard deviation is comparable to 1 in nearly all bins. 


\begin{tabular}{|c|c|c|c|c|}
\hline Sample & Simulation & Reweighting & Uncertainty & Abs. Difference \\
\hline 1 & 38277 & 38341.9 & $\pm 87.4_{-13.3}^{+12.0}$ & -64.9 \\
\hline 2 & 38400 & 38292.6 & $\pm 87.5_{-13.3}^{+11.9}$ & 107.4 \\
\hline 3 & 38406 & 38319.5 & $\pm 87.3_{-13.2}^{+11.9}$ & 86.5 \\
\hline 4 & 38686 & 38664.8 & $\pm 87.6_{-13.3}^{+12.0}$ & 21.2 \\
\hline 5 & 38265 & 38272.8 & $\pm 87.5_{-13.2}^{+11.9}$ & -7.8 \\
\hline 6 & 38407 & 38388.2 & $\pm 87.7_{-13.3}^{+12.0}$ & 18.8 \\
\hline 7 & 38405 & 38391.4 & $\pm 87.5_{-13.3}^{+12.0}$ & 13.6 \\
\hline 8 & 38625 & 38512.9 & $\pm 87.6_{-13.4}^{+12.0}$ & 112.1 \\
\hline 9 & 38399 & 38411.6 & $\pm 87.5_{-13.3}^{+12.0}$ & -12.6 \\
\hline 10 & 38358 & 38391.2 & $\pm 87.4_{-13.3}^{+12.0}$ & -33.2 \\
\hline 11 & 38577 & 38513.5 & $\pm 87.4_{-13.3}^{+12.0}$ & 63.5 \\
\hline 12 & 38311 & 38419.8 & $\pm 87.4_{-13.3}^{+11.9}$ & -108.8 \\
\hline 13 & 38539 & 38460.5 & $\pm 87.5_{-13.3}^{+12.0}$ & 78.5 \\
\hline 14 & 38633 & 38653.7 & $\pm 87.7_{-13.4}^{+12.0}$ & -20.7 \\
\hline 15 & 38620 & 38611.5 & $\pm 87.3_{-13.3}^{+11.9}$ & 8.5 \\
\hline 16 & 38293 & 38318.2 & $\pm 87.1_{-13.2}^{+11.9}$ & -25.2 \\
\hline 17 & 38559 & 38603.2 & $\pm 87.7_{-13.4}^{+12.0}$ & -44.2 \\
\hline 18 & 38339 & 38342.1 & $\pm 87.2_{-13.2}^{+11.9}$ & -3.1 \\
\hline 19 & 38478 & 38373.8 & $\pm 87.4_{-13.2}^{+11.9}$ & 104.2 \\
\hline 20 & 38462 & 38491.0 & $\pm 87.5_{-13.3}^{+12.0}$ & -29.0 \\
\hline
\end{tabular}

Table 5.1: Result of the closure test using $20 \mathrm{~W} \rightarrow \mu \nu$ samples. The quoted uncertainties reflect the binomial distribution accounting the trigger decision (first term) and the statistically limited efficiency measurements (second term). The asymmetric Gaussian approximation was used to calculate the second part of the uncertainties.

\begin{tabular}{c|c|c|c|c} 
Sample & Simulation & Reweighting & Uncertainty & Abs. Difference \\
\hline 1 & 1281 & 1285.4 & $\pm 7.6 \pm 0.34$ & -4.4 \\
2 & 1199 & 1216.0 & $\pm 7.5 \pm 0.32$ & -17.0 \\
3 & 1266 & 1277.9 & $\pm 7.8 \pm 0.32$ & -11.9 \\
4 & 1262 & 1264.1 & $\pm 7.8 \pm 0.33$ & -2.0 \\
5 & 1314 & 1317.0 & $\pm 8.1 \pm 0.36$ & -3.0 \\
6 & 1233 & 1243.7 & $\pm 7.7 \pm 0.32$ & -10.7 \\
7 & 1240 & 1247.0 & $\pm 7.4 \pm 0.32$ & -7.0 \\
8 & 1241 & 1247.2 & $\pm 7.7 \pm 0.33$ & -6.2 \\
9 & 1291 & 1295.1 & $\pm 7.8 \pm 0.33$ & -4.1 \\
10 & 1247 & 1248.1 & $\pm 7.6 \pm 0.33$ & -1.1 \\
11 & 1222 & 1223.6 & $\pm 7.5 \pm 0.32$ & -1.6 \\
12 & 1297 & 1300.4 & $\pm 7.7 \pm 0.33$ & -3.4 \\
13 & 1297 & 1288.9 & $\pm 7.9 \pm 0.32$ & 8.1 \\
14 & 1179 & 1188.2 & $\pm 7.6 \pm 0.33$ & -9.2 \\
15 & 1291 & 1281.3 & $\pm 7.7 \pm 0.34$ & 9.7 \\
16 & 1229 & 1232.5 & $\pm 7.8 \pm 0.34$ & -3.5 \\
17 & 1225 & 1228.7 & $\pm 7.3 \pm 0.30$ & -3.7 \\
18 & 1232 & 1237.0 & $\pm 7.7 \pm 0.32$ & -5.0 \\
19 & 1248 & 1237.9 & $\pm 7.6 \pm 0.32$ & 10.1 \\
20 & 1266 & 1268.8 & $\pm 7.6 \pm 0.33$ & -2.8
\end{tabular}

Table 5.2: Result of the closure test using 20 inclusive $W W$ samples requiring exactly 2 muons in the final state. Again, two sources of the uncertainties were considered, as quoted in the table. Here, the symmetric Gaussian approximation was used. On average the reweighting approach overestimates the event yield, as expected. 
In addition to the $W \rightarrow \mu \nu$ sample, the re-weighting procedure has been tested on several other samples with different physics content. Most of those samples provide much less statistics, such that an extensive test as the one performed for the $W$ sample could not be performed. The uncertainty on the sum of event weights is given as a symmetric approximation for all further tests. Furthermore, since the trigger re-weighting technique has mainly been used in searches for supersymmetry so far, it is the absolute event count that is of highest interest here, rather than distributions in the muon variables. In Figure 5.8 the results for a closure test on a $W W$ sample requesting exactly two muons in the final state is shown. For this sample, the expectation value calculated using the trigger re-weighting procedure tends to be slightly smaller than the event counts obtained by using the trigger simulation for nearly all samples, as is also clear from the numbers in Table 5.2.

Apart from this being a systematic effect of the trigger re-weighting, there is another caveat for this comparison. It is usually a reasonable approximation that the efficiency of a muon trigger is roughly flat in the muon transverse momentum for values significantly above the threshold. If as in the case of muon triggers the efficiencies are measured from a well defined physics process, the phase-space in which such a measurement can be performed is defined by that physics process. For the process $Z \rightarrow \mu \mu$ the $p_{T}$ spectrum of the muons has a relatively sharp drop at $p_{T} \sim 45$ $\mathrm{GeV}$, such that a single bin in $p_{T}$ is used for the efficiency of a muon trigger for muons with larger transverse momenta.

The samples that have been used for this study are a part of the mc10b ATLAS official MC production. In these samples, because faulty alignment constants for the muon system have been used, the trigger efficiency for muons in the barrel region decreases with increasing values of the transverse momentum. This effect has been observed to become significant for muons with $p_{T}>40 \mathrm{GeV}$, roughly. For the particular trigger chain that has been used for this closure test, the efficiency was observed to drop by roughly $30 \%$ between $p_{T}=40 \mathrm{GeV}$ and $p_{T}=500$ $\mathrm{GeV}$. The phase-space occupied by muons from a $W$ boson decay is comparable to that occupied by muons from a $Z$ boson decay (cf. Figure 5.5(a)), but in the $W W$ sample there is a significant fraction of muons with a transverse momentum larger than $50 \mathrm{GeV}$ as shown in Figure 5.8(c). Since the drop in the efficiency cannot be resolved by the Tag and Probe method due to a lack of statistics in the high $p_{T}$ regime, the trigger weights are expected to overestimate the actual event count using the trigger simulation in this sample. This is in fact observed - as indicated by Figure 5.8(c), for $p_{T}>50 \mathrm{GeV}$, the trigger simulation nearly consistently gives a lower event yield than the trigger re-weighting, where a trend to larger differences with increasing $p_{T}$ values can be observed. Such a behaviour is not observed in the $\eta$ distribution, as seen in Figure 5.8(d).

In the faulty MC production, the efficiency decreases roughly linear with increasing transverse momentum. To roughly estimate the effect that this defect in the $m c 10 b$ production has on the total event yields for several physics samples, a simple procedure has been used. Assuming a linear behaviour of the efficiency drop, based on an average trigger efficiency of $72 \%$ in the barrel region, at $p_{T}=40 \mathrm{GeV}$ and an efficiency of $42 \%$ at $p_{T}=500 \mathrm{GeV}$, the average trigger efficiency at $p_{T}=$ a) $50 \mathrm{GeV}$, b) $90 \mathrm{GeV}$, c) $130 \mathrm{GeV}$ and d) $170 \mathrm{GeV}$ has been calculated, and for each physics sample these average efficiencies have been weighted with the fraction of central muons in that sample with $p_{T} \in$ a) $[40 \mathrm{GeV}, 80 \mathrm{GeV})$, b) $[80 \mathrm{GeV}, 120 \mathrm{GeV}$ ), c) $[120 \mathrm{GeV}, 160 \mathrm{GeV})$ and d) $[160 \mathrm{GeV}, \infty)$ to calculate a new average efficiency in the barrel. The new average has been compared to the $72 \%$. This estimate of the effect does not take into account the underlying $\eta$ distributions in the samples, and in the case of more than one muon in the final state the estimate is based solely on the leading muon, as the fraction of subleading muons with transverse momenta in the badly modelled region is small. For the $W W$ sample, the effect has been estimated to cause a decrease of about $0.2 \%$ in the total event yield, where 


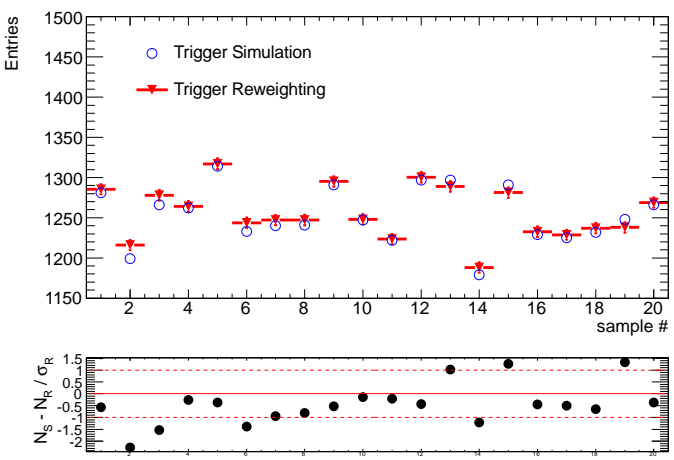

(a)
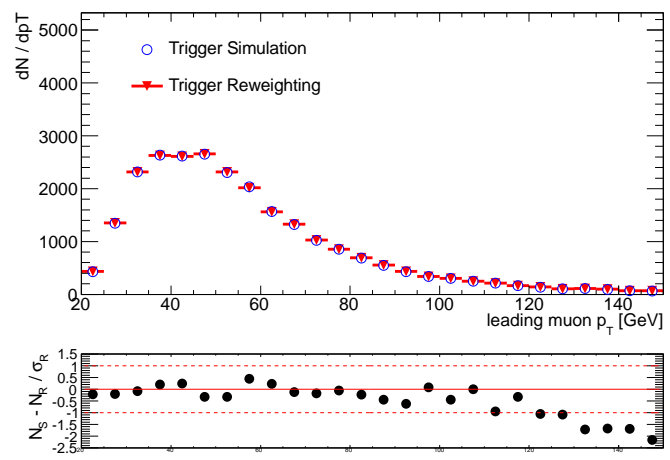

(c)

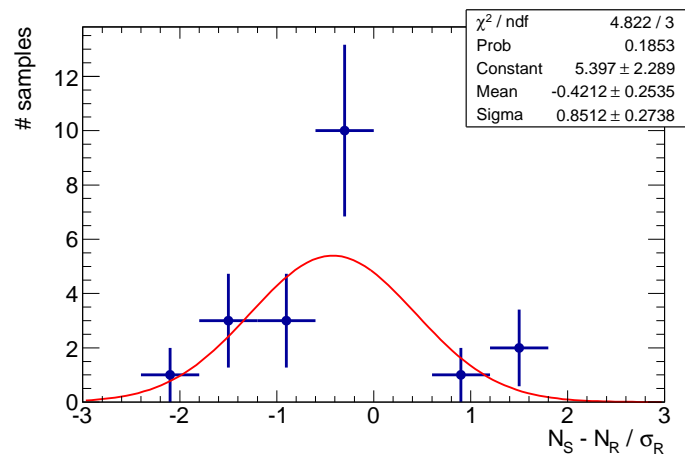

(b)

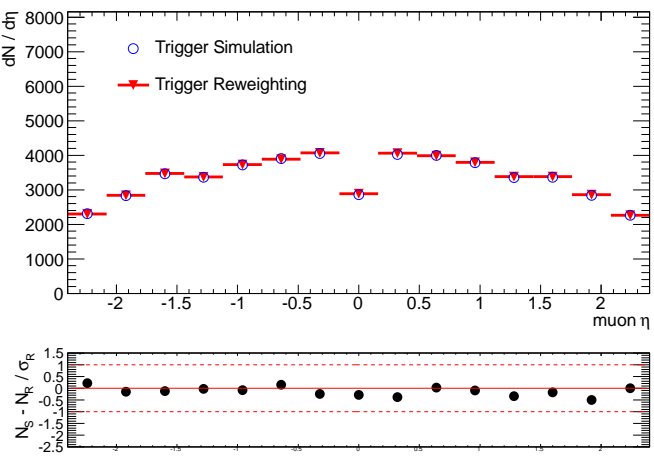

(d)

Figure 5.8: A comparison of some distributions for the closure test with exactly two muons in the final state. In (a), the total event yield is shown as a function of the sample number, for the run using the trigger simulation (blue circles) and for the run using the trigger re-weighting (red triangles). In nearly all samples, the trigger re-weighting predicts a higher event yield, as is emphasised by the pull distribution shown in (b). In (c), for the sum of all 20 samples, the distribution of the transverse momentum for the leading lepton is shown. The plot shows that for $p_{T}^{1} \gtrsim 80 \mathrm{GeV}$ the trigger simulation yields sytematicaly smaller event yields than the trigger re-weighting, while for $p_{T} \lesssim 80 \mathrm{GeV}$ such a behaviour is not observed. The difference tends to increase with increasing transverse momentum. Such a behaviour is also not observed in the sum of the $\eta$ distributions of both muons, as shown in (d). The observed behaviour with respect to the transverse momentum can most likely be attributed to a bug in the MC muon trigger simulation at large transverse momenta.

the spread of this estimate over the 20 samples used is negligible. This corresponds to 2 to 3 events per subsample, which by comparison to the numbers in Table 5.2 reduces the observed bias noticeably, but there is still a small systematic shift which can then be attributed to the re-weighting procedure with the used efficiencies and binning.

As a final result, the absolute event yields and the sum of weights for other physics samples with different muon multiplicities in the final state are summarised in Table 5.3. The overall agreement between the event count using the trigger simulation and the sum of weights in each sample is again good. The largest deviations are seen in the single muon channels, where the systematic due to the used binning is expected to be most noticable. In all cases, the relative 
difference between the integer event yield and the sum of weights is less than $1.1 \%$.

\begin{tabular}{c|c|c|c|c|c|c} 
Sample & $n_{\text {Muons }}$ & Simulation & Reweighting & Uncertainty & Abs. Difference & estim. Bug Effect \\
\hline SU4 & 1 & 3590 & 3516.5 & $\pm 2.4 \pm 28.8$ & 73.5 & $0.6 \%$ \\
SU4 & 2 & 276 & 280.0 & $\pm 0.2 \pm 4.1$ & -4.0 & $0.3 \%$ \\
SU4 & 3 & 8 & 7.9 & $\pm 0.0 \pm 0.4$ & -0.1 & $0.2 \%$ \\
WZ & 1 & 55365 & 54756.0 & $\pm 25.8 \pm 108.8$ & 609.0 & $0.6 \%$ \\
WZ & 2 & 11359 & 11390.0 & $\pm 2.3 \pm 23.6$ & -31.0 & $0.2 \%$ \\
WZ & 3 & 783 & 784.3 & $\pm 0.1 \pm 2.8$ & -1.3 & $0.1 \%$ \\
ZZ & 2 & 33231 & 33342.2 & $\pm 6.3 \pm 40.9$ & -111.2 & $0.2 \%$ \\
$\mathrm{t} \bar{t}$ & 1 & 43308 & 42926.0 & $\pm 33.5 \pm 100.1$ & 382.0 & $0.7 \%$ \\
$\mathrm{t} \bar{t}$ & 2 & 2208 & 2214.7 & $\pm 0.8 \pm 10.89$ & -6.7 & $0.3 \%$
\end{tabular}

Table 5.3: Outcome of the sanity checks using various samples and requiring various muon multiplicities in the final state. The overall agreement is good, however in particular in the 1 muon channels relatively large differences are observed. These are of the order of $1 \%$ and can most likely be accounted to the used binning. Note that the estimated effect of the defect can only give a very rough idea of the actual impact, which tends to increase the discrepancy in the 1 muon channels while it tends to decrease the discrepancies in the channels with more than 1 muon in the final state.

\section{Closure Test for Uncertainties on Event Weights}

After testing the general performance of the trigger re-weighting procedure, in a second step the goodness of the approximation (5.34) is assessed. In a first simple test, the actual correlation coefficient between two event weights is compared to the approximation that has been proposed for the sum of trigger weights. In a second step, a MC sample for a well defined physics scenario with four leptons in the final state is subject to an ensemble test, imposing a trigger requirement on one, two, three or all four leptons in the final state with different trigger combinations.

Two event weights $W_{1}$ and $W_{2}$ are considered as a function of up to four independent estimators for a trigger efficiency, $\varepsilon_{1}, \varepsilon_{2}, \varepsilon_{3}$ and $\varepsilon_{4}$. In order to assess the quality of assumption (5.29), the distributions of the efficiencies that have been used to sample the distribution functions for the weights are in this case given by equation (5.5). Figures 5.9(a), 5.9(b) and 5.9(c) illustrate how the Gaussian approximation performs for different absolute efficiencies and statistics. In general the approximation works best for efficiencies close to 0.5 and large statistics, but for most applications in ATLAS the available statistics are sufficient to justify the usage of the approximation.

The simplest, non-trivial scenario is given by an analysis using a single muon trigger with two muons above the threshold in the final state. Assuming that the two muons in each event have sufficiently different kinematics such that equation (5.30) holds, the event weights for two such events are given by

$$
\begin{aligned}
& W_{1}=1-\left(1-\varepsilon_{i}\right)\left(1-\varepsilon_{j}\right) \\
& W_{2}=1-\left(1-\varepsilon_{k}\right)\left(1-\varepsilon_{l}\right)
\end{aligned}
$$

where $i \neq j$ and $k \neq l$. In the case that also $i, j \in\{k, l\}$ the covariance between weights $W_{1}$ and $W_{2}$ is 0 in accordance with equation (5.34). If $i=k$ and $j=l$ the two weights are $100 \%$ correlated and as discussed above the equation (5.34) will underestimate this coefficient. The more relevant case is if $i=k$, but $j \neq l$, such that the correlation factor is not 0 or 1 , but it 


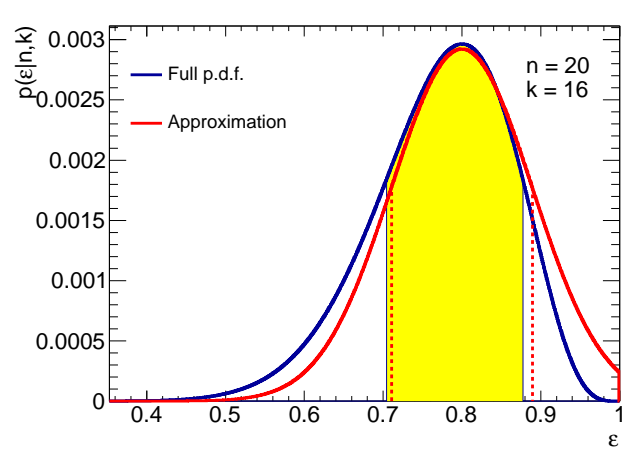

(a)

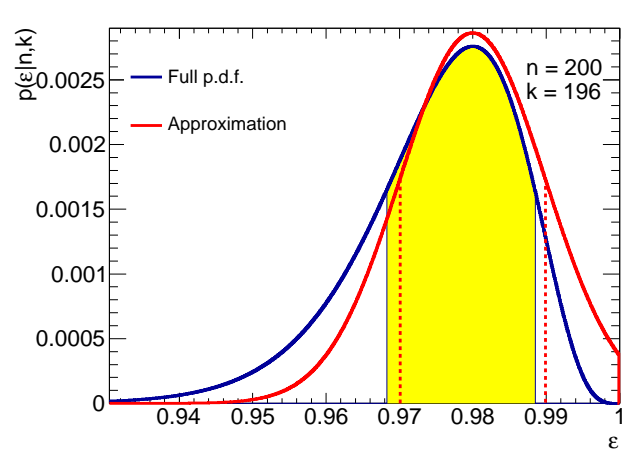

(c)

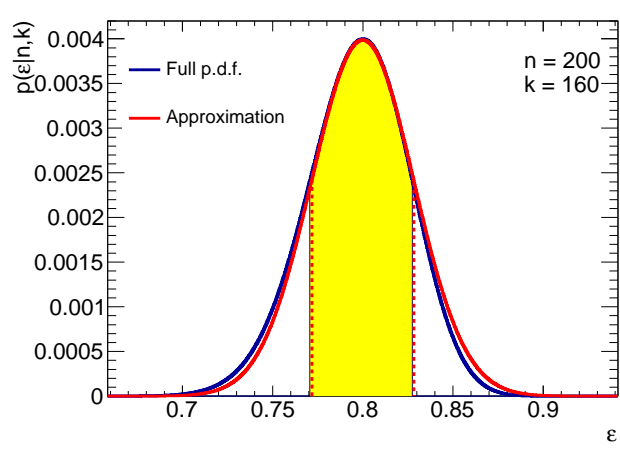

(b)

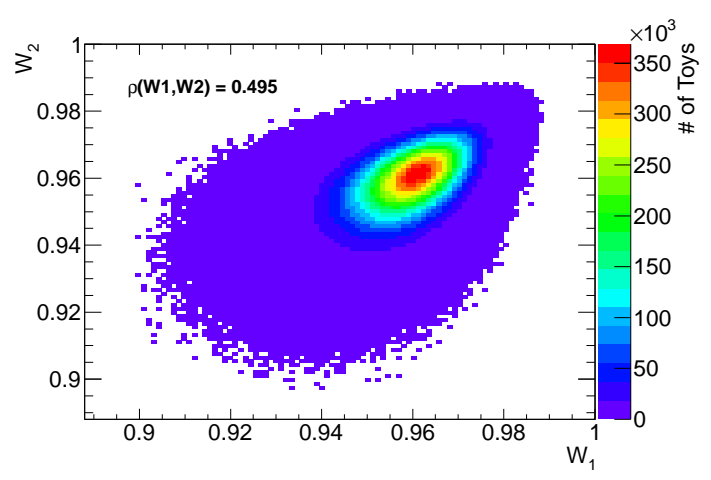

(d)

Figure 5.9: In (a) to (c) the Bayesian p.d.f. (5.5) is compared to the Gaussian approximation. In (a) the p.d.f.s are shown for a measurement with small statistics, and an efficiency of roughly $80 \%$. In (b) the p.d.f.s for a similar measurement with increased statistics and in (c) the p.d.f.s for an efficiency close to $100 \%$ are plotted. While for the measurement with small statistics and for the measurement of an efficiency close to $100 \%$ in the Gaussian approximation the domain of the p.d.f. is continously extended to values larger than 1, which is unphysical, the estimated uncertainties are still in reasonable agreement with the boundaries of the smallest interval covering $68 \%$ of the full p.d.f.. If the efficiency differs sufficiently from 1 and if enough statistics are available for the measurement, the Gaussian approximation works very well. Using the full p.d.f.s, the plot in (d) shows the two-dimensional distribution of two weights $W_{1}$ and $W_{2}$ for a final state with two muons. The correlation coefficient between the two weights is estimated using a total of 100 million pseudo experiments.

will depend on both the central values and the uncertainties on the three different efficiencies. Assuming a realistic value of $\varepsilon_{i, j, k, l}=80 \%$ and different statistics for the measurements, the correlation coefficient $\varrho\left(W_{1}, W_{2}\right)$ can be estimated using pseudo experiments. In order to keep the statistical fluctuations at a minimum, for each test that has been performed in this context a total of 100 million pseudo experiments have been used. 
Figure 5.9(d) shows the two dimensional distribution for the weights $W_{1}$ and $W_{2}$ for the case

$$
\begin{aligned}
\varepsilon_{1} & =\frac{160}{200}=0.8_{-0.029}^{+0.027} \approx 0.8 \pm 0.028, \\
\varepsilon_{2} & =\frac{160}{200}=0.8_{-0.029}^{+0.027} \approx 0.8 \pm 0.028, \\
\varepsilon_{3} & =\frac{160}{200}=0.8_{-0.029}^{+0.027} \approx 0.8 \pm 0.028, \\
W_{1} & =1-\left(1-\varepsilon_{1}\right)\left(1-\varepsilon_{2}\right) \approx 0.96 \pm 0.008, \\
W_{2} & =1-\left(1-\varepsilon_{1}\right)\left(1-\varepsilon_{3}\right) \approx 0.96 \pm 0.008,
\end{aligned}
$$

where equation (5.34) estimates the correlation coefficient to be

$$
\varrho_{\text {approx }}\left(W_{1}, W_{2}\right)=\frac{\operatorname{cov}\left(W_{1}, W_{2}\right)}{\sigma_{W_{1}} \sigma_{W_{2}}}=0.5 .
$$

From the histogram, this number is estimated to be $\varrho_{\text {hist }}\left(W_{1}, W_{2}\right)=0.495$, which is close to the approximation.

The quality of equation (5.34) has been tested for more configurations and the outcome of the performed tests are summarised in Figure 5.10. Figure 5.10(a) shows the absolute difference $\varrho_{\text {hist }}-\varrho_{\text {approx }}$ for final states with two muons, using a single muon trigger, such that $W_{1}$ and $W_{2}$ are defined as above, for the cases

$$
\begin{array}{ll}
\varepsilon_{1} & \in[0.05,0.95] \\
\varepsilon_{2} & =0.7 \pm 0.03 \\
\varepsilon_{3} & =0.7 \pm 0.03
\end{array} \quad \text { where } \varepsilon_{1}=\frac{k}{n} \text { with } n \in[20,400]
$$

Figure 5.10(b) shows the same for a final state with three leptons, where

$$
\begin{aligned}
& W_{1}=1-\left(1-\varepsilon_{1}\right)\left(1-\varepsilon_{2}\right)\left(1-\varepsilon_{3}\right) \quad \text { and } \\
& W_{2}=1-\left(1-\varepsilon_{1}\right)\left(1-\varepsilon_{2}\right)\left(1-\varepsilon_{4}\right),
\end{aligned}
$$

using the same values of $\varepsilon_{1}, \varepsilon_{2}, \varepsilon_{3}$ and $\varepsilon_{4}=0.75 \pm 0.03$. As shown by the two plots, the maximum deviation between the approximation and the actual correlation factor between the two event weights $W_{1}$ and $W_{2}$ appears for small statistics $n$ and efficiencies close to 0 or close to 1 , where the approximation can be off by as much as $6 \%$. For the majority of realistic cases, where $n \gtrsim 100$ and $0.5 \lesssim \varepsilon \lesssim 1$. the approximation works well, with a maximum deviation of about 0.02 in the correlation coefficient.

As a final test, the approximation is tested using a MC sample modelling the associate production of a $\tilde{\chi}_{2}^{0}$ and a $\tilde{\chi}_{3}^{0}$ with four leptons in the final state, cf. chapter 6 . For this test, the MC truth four-vectors of all leptons in an event have been used to calculate the event weight and the uncertainty on it. After a first run where the nominal efficiencies have been used, a series of pseudo experiments has been run, where the trigger efficiencies are smeared according to their p.d.f..

It must be emphasised that this is a test of the uncertainty on the sum of weights rather than a test for the central value - the estimator for the expectation value is the sum of all event weights calculated using the most probable value for each trigger efficiency. According to the central limit theorem, the distribution of the sum of weights calculated from the pseudo experiments 


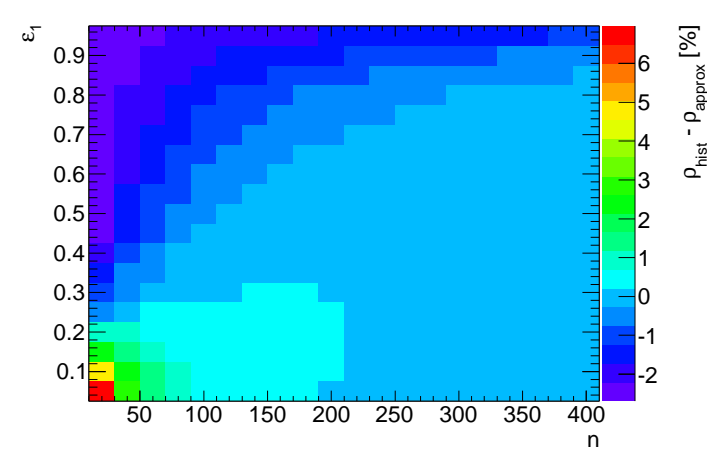

(a)

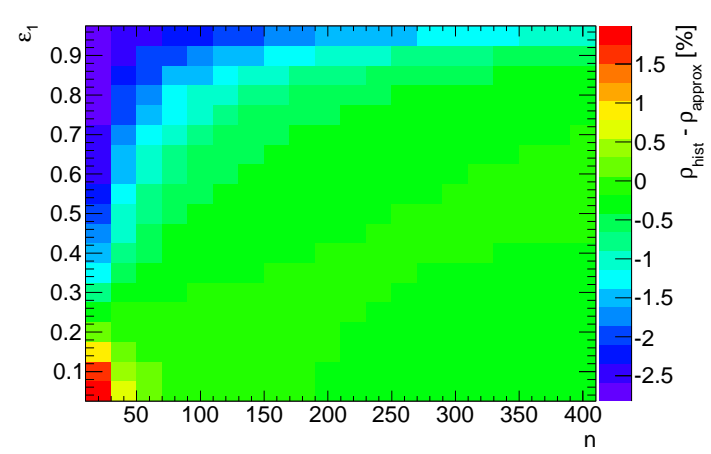

(b)

Figure 5.10: The correlation coefficient calculated using the approximation (5.34) is compared to the correlation coefficient estimated by using toy experiments. In (a), the difference of the two correlation coefficients for two weights covering two muons in the final state, where the kinematic properties of the first muon in each event are the same, is shown as a function of the trigger efficiency for that muon and the statistics that are available for the measurement of that efficiency. The agreement is worst for small statistics and efficiencies close to 0 or 1 , as expected. In (b) the same comparison is shown for event weights covering three muons in the final state, where the first muon has the same kinematic properties in each event. Again, the agreement is worst for very small or very high efficiencies and small statistics.

will be roughly a Gaussian, where the mean value $\mu_{S}$ is the sum of the mean values of the individual summands. This will in general differ from the estimator for the sum of weights $S$, because equations (5.29) and (5.30) are only an approximation. The difference between $S$ and $\mu_{S}$ is expected to be small; however, as another test for the approximations (5.29) and (5.30) the ensemble test discussed here has been performed two times using different p.d.f.s for the trigger efficiencies, as is explained below.

A total of 10,000 events have been used for the ensemble test. Each event has four leptons in the final state, all of which are considered as muons for this test. Three different trigger efficiencies are used to calculate the sum of weights for different trigger configurations. These efficiencies are $\varepsilon_{\mathrm{SM}}$, which is the absolute efficiency of a single muon trigger, $\varepsilon_{\mathrm{DM}}$ which is the absolute efficiency of one leg of a symmetric di-muon trigger, and $\varepsilon_{\mathrm{DMC}}$, which is the efficiency of the same di-muon trigger for a muon that has failed the trigger hypothesis of the single muon trigger. The efficiencies are considered as functions of the muon $p_{T}$, the muon $\eta$ and the muon $\phi$, and a total of 3060 bins have been used. The efficiencies have been fixed to

$$
\begin{aligned}
& \varepsilon_{\mathrm{SM}}=160 / 200=0.8_{-0.029}^{+0.027} \approx 0.800 \pm 0.028 \quad \text { for } p_{T}>20 \mathrm{GeV} \\
& \varepsilon_{\mathrm{DM}}=140 / 200=0.7_{-0.033}^{+0.031} \approx 0.700 \pm 0.032 \quad \text { for } p_{T}>10 \mathrm{GeV} \\
& \varepsilon_{\mathrm{DMC}}=\begin{array}{rr}
140 / 200 & =0.7_{-0.033}^{+0.031} \approx 0.700 \pm 0.032 \text { for } 10 \mathrm{GeV}<p_{T} \leq 20 \mathrm{GeV} \\
60 / 200 & =0.3_{-0.031}^{+0.033} \approx 0.300 \pm 0.032 \text { for } \\
p_{T}>20 \mathrm{GeV},
\end{array}
\end{aligned}
$$

which concerning the statistics is roughly a realistic case. A basic cut-flow is applied to the events, where all four leptons are required to pass simple kinematic cuts $\left(|\eta|<2.4\right.$ and $p_{T}>10$ $\mathrm{GeV}$ ). Eight different trigger configurations are considered for this test:

(a) single muon trigger, using the leading muon only, 
(b) single muon trigger, using the leading and the subleading muon,

(c) single muon trigger, using the leading, subleading and third leading muon,

(d) single muon trigger, using all four muons,

(e) symmetric di-muon trigger, using the leading and subleading muon only,

(f) symmetric di-muon trigger, using the leading, subleading and third leading muon,

(g) symmetric di-muon trigger, using all four muons,

(h) logical OR of single muon trigger with the symmetric di-muon trigger, using the three leading muons.

In a first run, the estimator for the sum of weights and the uncertainty on that sum of weights is calculated using the nominal values of all efficiencies and the approximation (5.34). In a second step a total of 1,000 pseudo experiments is processed. Each efficiency is smeared according to a Gaussian distribution where the mean value is given by the nominal efficiency and the width is given by the symmetric approximation of the uncertainties on these efficiencies. After smearing, the 10,000 events are re-processed and the weights for all eight cases are re-calculated using the smeared efficiency. In a third step this procedure is repeated, but using the p.d.f.s (5.5).

The outcome of this test is summarised in Figure 5.11, Figure 5.12 and Table 5.4. The plots in Figure 5.11 and Figure 5.12 show the distribution of the sum of weights for each of the eight cases, where a Gaussian distribution is fitted to that distribution and both ensembles are considered. In addition to the mean value and the width as calculated from the first run without smearing the efficiencies, the estimated uncertainties assuming fully correlated efficiency measurements and completely neglecting any correlation between the event weights is shown.

The deviations between the two ensembles are small, although there is a clear difference in the mean values of both distributions, as expected. In general, the uncertainty on the sum of weights is best estimated using the approximation (5.34). Varying all efficiencies simultaneously in the same direction $(\varrho=1)$ overestimates the uncertainty by a large factor usually, while assuming that all event weights are completetly uncorrelated $(\varrho=0)$ systematically underestimates the uncertainties. Although as expected the agreement between the calculated uncertainty and the width of both distributions is not perfect, this test shows that for a realistic physics sample the correlations between event weights are typically too large to completely neglect them, but the correlation coefficients between two events are on average by far different from 1 . In the context of trigger weights, it is therefore necessary to make use of equation (5.34) to calculate an uncertainty on the sum of weights that describes well the actual width of the distribution. 


\begin{tabular}{c|c|c|c|c|c|c} 
Trigger & Muons & Estimate & Gaussian P.D.F. & Full P.D.F. & $\varrho_{i j}=0$ & $\varrho_{i j}=1$ \\
\hline single muon & 1 & $4530.4 \pm 10.0$ & $10.5 \pm 0.2$ & $10.0 \pm 0.2$ & 2.1 & 160.2 \\
single muon & 2 & $5423.0 \pm 3.3$ & $3.5 \pm 0.1$ & $3.4 \pm 0.1$ & 0.7 & 65.5 \\
single muon & 3 & $5575.6 \pm 1.2$ & $1.2 \pm 0.0$ & $1.2 \pm 0.0$ & 0.4 & 27.8 \\
single muon & 4 & $5591.1 \pm 0.9$ & $1.0 \pm 0.0$ & $1.0 \pm 0.0$ & 0.4 & 21.8 \\
di-muon & 2 & $2776.4 \pm 13.1$ & $13.1 \pm 0.3$ & $13.5 \pm 0.3$ & 2.4 & 257.0 \\
di-muon & 3 & $4442.1 \pm 9.4$ & $9.5 \pm 0.2$ & $9.4 \pm 0.2$ & 1.8 & 231.3 \\
di-muon & 4 & $5191.8 \pm 4.6$ & $4.7 \pm 0.1$ & $4.7 \pm 0.1$ & 0.9 & 138.8 \\
OR of both & 3 & $5632.6 \pm 0.4$ & $0.4 \pm 0.0$ & $0.4 \pm 0.0$ & 0.2 & 9.5
\end{tabular}

Table 5.4: Results for the test of equation (5.34) for different triggers and different muon multiplicities in the final state.

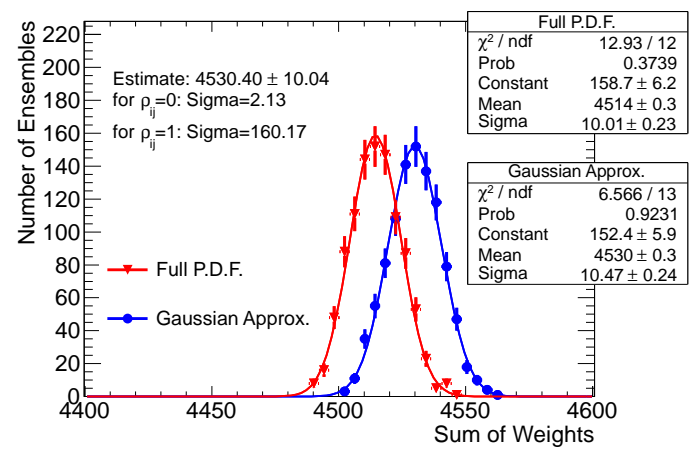

(a) single muon trigger, one muon

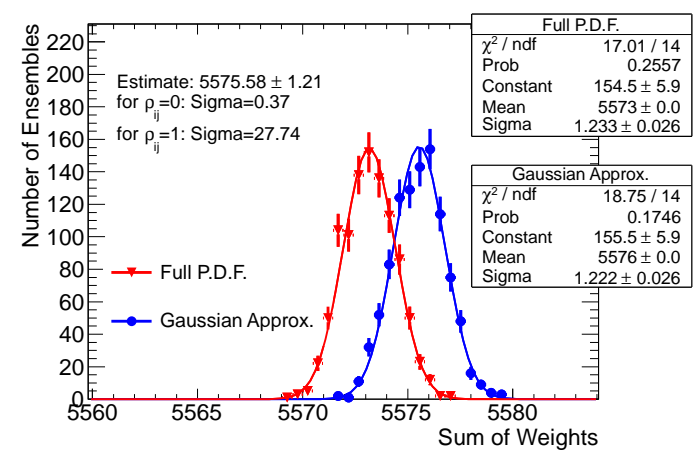

(c) single muon trigger, three muons

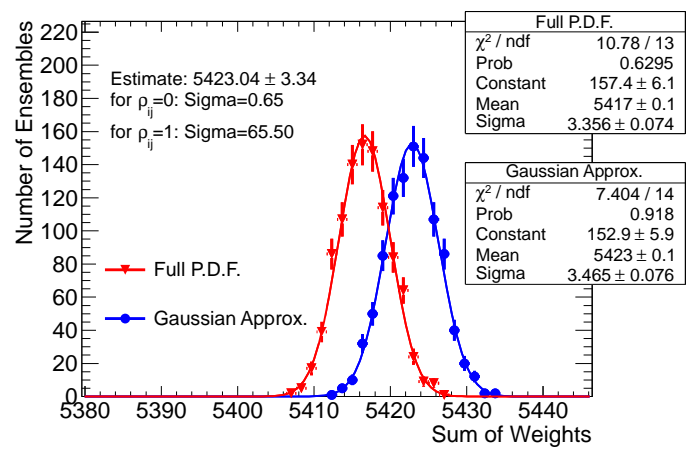

(b) single muon trigger, two muons

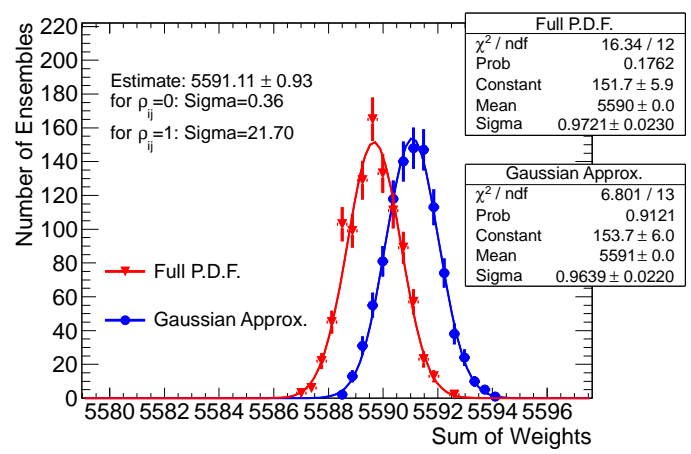

(d) single muon trigger, four muons

Figure 5.11: Results of the ensemble test using a SUSY physics sample for a single muon trigger and different muon multiplicities. The plots show the distribution of the sum of weights, using a Gaussian approximation for the p.d.f. of the trigger efficiency in each bin and using the full p.d.f. as derived in section 5.2.3. The estimate (using the Gaussian p.d.f.s) for the sum of weights and the uncertainty on that sum that has been calculated using equation (5.34) is shown in the top left of each plot. In addition, the uncertainty on the sum of weights is given under the assumption that all the uncertainties on all efficiencies are fully uncorrelated $\left(\varrho_{i j}=0\right)$ or fully correlated $\left(\varrho_{i j}=1\right)$. The three given uncertainties should be compared to the widths of the distribution for the Gaussian approximation that are shown in each plot. In all cases the best estimate is achieved if equation (5.34) is used. 


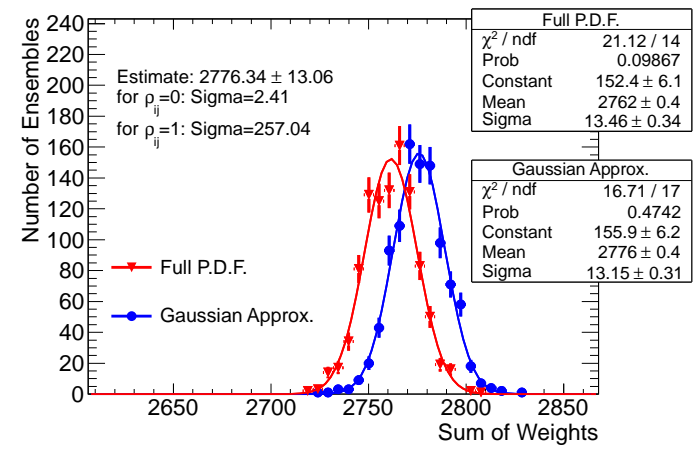

(a) di-muon trigger, two muons

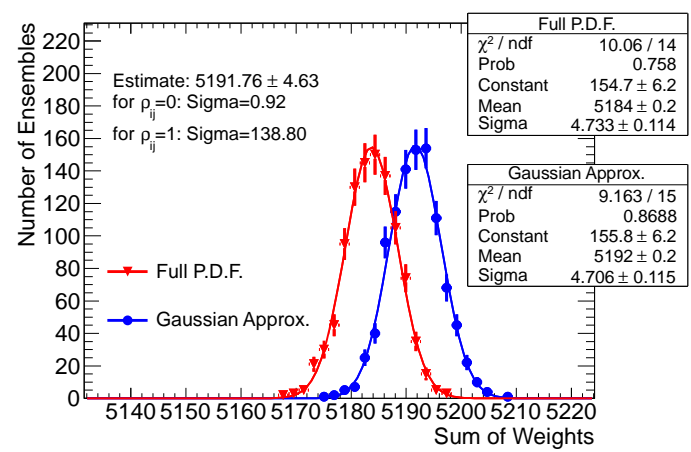

(c) di-muon trigger, four muons

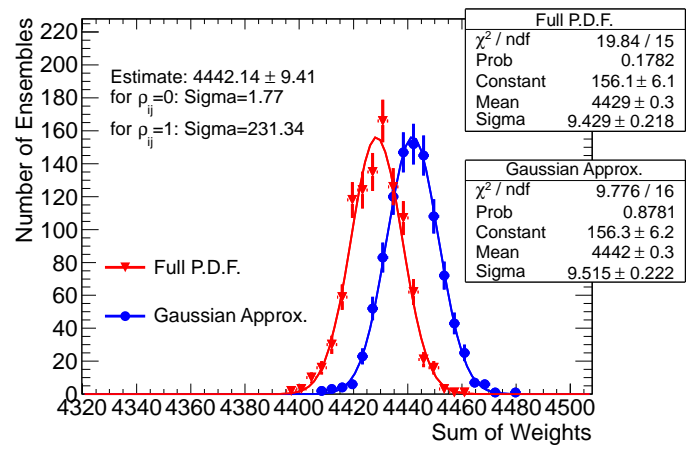

(b) di-muon trigger, three muons

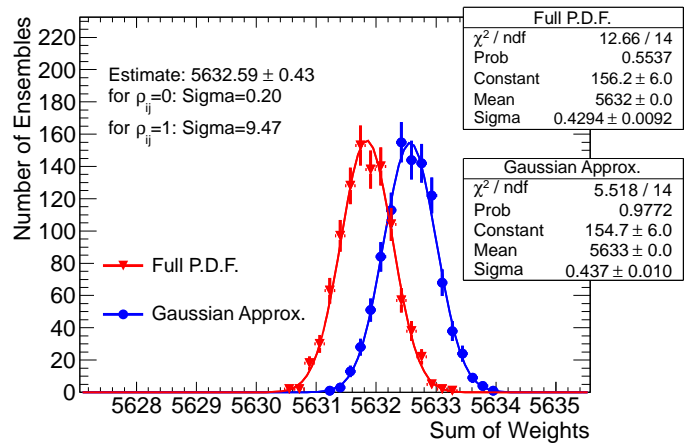

(d) OR of single muon and di-muon trigger, three muons

Figure 5.12: The results from ensemble test using a SUSY physics sample for di-muon triggers and a combination of a di-muon trigger with a single muon trigger. 


\section{Summary of Closure Tests}

All tests that have been performed show that the presented methods can be used to replace the simulation of the muon triggers in the ATLAS MC. With the used granularity in the measurement of the trigger efficiencies the observed biases are roughly at the order of $1 \%$ in all distributions that have been examined, even if the physics contents in some samples differ significantly from the physics content in the sample that has been used to measure the efficiency. The magnitude of the observed bias is in all cases roughly comparable to the statistical uncertainty.

The tests performed for equation (5.34) have shown that the linear approximation for the calculation of the covariance between two event weights works excellent for the majority of the relevant cases. It allows for a more reasonable approximation of the uncertainty on the sum of weights than either of the simple approaches that are commonly used.

\subsection{Performance of the ATLAS Muon Triggers during the $\sqrt{s}=7$ TeV Run}

During the LHC run in 2011 the instantaneous luminosity has constantly been increased such that for analyses targeting final states with muons the primary muon triggers had to be adjusted to higher thresholds in order to keep the trigger rates at an acceptable level. The efficiencies of the triggers used in some searches for SUSY with two and more leptons in the final state have been measured using the 2011 dataset. Before the results of these measurements are shown, a short overview on the techniques for measuring muon trigger efficiencies is given.

\subsubsection{Techniques for Measuring Muon Trigger Efficiencies}

The goal for most techniques that can be used to measure the efficiency of some trigger is to select an unbiased sample of objects or events that are supposed to pass the hypothesis of that trigger and check what fraction of that sample actually did pass that hypothesis, i.e. an unbiased measurement of $n$ and $k$ as defined in section 5.2.

While in MC it is straight forward to perform such a measurement using all suitable events in some MC sample it is more difficult to select an unbiased sample in data because only events that have passed some trigger hypothesis are stored and the samples selected by the minimumbias trigger do usually not provide enough statistics for the measurements that are needed.

There are different ways to obtain a nearly unbiased sample from data that all rely on the same principle. The idea is to decouple the event selection that defines the number $n$ from the trigger decision that defines the number $k$. For a muon trigger, which selects events based on distinct objects, this can be achieved by either using a different trigger signature like electrons or jets, or by using the same trigger signature but using different objects for the event selection and the measurement of the trigger efficiency. Both methods will be shortly described here, but only the Tag and Probe method has been used for the measurements presented in this chapter.

\section{Orthogonal Trigger Method}

With this method a data sample is selected using a trigger $B$ that is orthogonal to the muon trigger of interest $A$. Orthogonal means that the trigger decision for $A$ is completely uncorrelated to the trigger decision for $B$. Usually an electron or a jet trigger is a good choice here, although at some level there might be small correlations between the trigger decisions for different signatures 
- for instance for both electron and muon reconstruction at the EF tracking information is used. Events are selected if they pass the trigger $B$ and all muons in the selected events are used to calculate the efficiency of the muon trigger $A$.

One shortcoming of this method can be the limited statistics. If for instance $B$ is an electron trigger, events with both a well reconstructed electron and a well reconstructed muon do not occur at high rates, in particular if due to the high rate of events with single, low $p_{T}$ electrons the thresholds for the available electron triggers are high. In order to increase the statistics of the test sample, a selection of triggers $\left\{B_{i}\right\}$ can be used instead of a single trigger $B$, but for an unbiased measurement it must be ensured that all of these triggers are orthogonal to the muon trigger $A$. Without any further selection criteria - that will reduce the available statistics again - it is also difficult to measure the efficiency of the muon trigger $A$ on a well defined sample, as depending on the set of orthogonal triggers events with very different physics content are selected.

\section{Tag and Probe Method}

This method selects events based on the same or a signature very similar to the one used by the trigger $A$. For the measurement of a muon trigger efficiency, events with at least two muons are selected, and one of the muons in the event is required to pass some muon trigger hypothesis $B$, where $B$ can be equal to $A$, or not. This muon is referred to as the $\operatorname{tag}$ muon and this selection ensures that the event has been stored no matter what the evaluation of the trigger hypothesis $A$ on any of the other muons in the event yields. These remaining muons are referred to as probe muons, and the efficiency of the trigger $A$ can be measured by counting the total number of probe muons (which is $n$ here) and the total number of probe muons that pass the hypothesis of the trigger $A$ (which is $k$ here) using equation (5.4). The method relies on the independence of the trigger decisions on two different muons, and in this sense the Tag and Probe method is a special case of the orthogonal trigger method.

Although there are ways to select samples with a well defined physics content using the orthogonal trigger method with different signatures, there is a natural candidate for such a physics process using the Tag and Probe method, which is the production of a $Z$ boson and the subsequent decay of that particle into two muons. The motivation for selecting a sample for the measurement of a trigger efficiency that is dominated by a well known and well defined physics process is that usually the measurement of some trigger efficiency depends on the sample it is measured from, as discussed above. Using a well defined sample for a measurement ensures comparability between different measurements of the same quantity which in the case of muon trigger efficiencies can be important if for instance two different analyses use different object selection criteria, but the same trigger. In such a case, the measurement of the trigger efficiency on a well defined sample allows for a direct study of the dependence of the trigger efficiency on the object selection criteria.

Candidate events for $p p \rightarrow Z X \rightarrow \mu \mu X$ are selected by requiring exactly two oppositely charged muons with an invariant mass close to the mass of the $Z$ boson, $\left|m_{\mu \mu}-m_{Z}\right|<\Delta_{m}$. The absolute value of $\Delta_{m}$ can be varied, where a larger value of $\Delta_{m}$ naturally yields larger statistics, but also a larger contamination with events from different physics processes. If that contamination can not be neglected, sometimes a background subtraction using MC is used, and sometimes a more accurate definition of $X$ is needed. 


\subsubsection{Performance of the Primary ATLAS Muon Triggers during the $\sqrt{s}=7$ TeV Run in 2011}

The ATLAS muon trigger efficiencies have been measured for the analyses searching for SUSY in final states with leptons using the data taken in 2011 [138, 139, 140, 141, 142, 143].

\section{Event Selection and Object Reconstruction}

Events are selected from the physics_Muons trigger stream and the object selection has been done following the criteria defined in the physics analysis, which are briefly described here; details on the reconstruction algorithms can for instance be found in [144].

Each event is demanded to pass the data quality requirements, to report no error after assessing the LAr quality, to contain no jet failing the jet quality criteria VeryLooseBad [145], and to contain at least one primary vertex with at least five associated tracks.

Jets are reconstructed making use of the anti- $k_{t}$ jet algorithm [146]. Topological clusters are used as an input and a distance parameter of $R=0.4$ has been used. A 'baseline' jet is defined as a jet candidate with a transverse momentum of more than $20 \mathrm{GeV}$ and it is required to lie within $|\eta|<4.9$.

Electrons are reconstructed using the egamma algorithm. A 'baseline' electron is defined as an electron candidate with a transverse energy of more than $10 \mathrm{GeV}$ and $|\eta|<2.47$. The electron $\eta$ and $\phi$ is calculated either from the track of the electron or, if there are not enough hits in the pixel detector and the SCT, from the respective coordinates of the cluster in the ECAL. A baseline electron is as well required to pass mediumPP quality criteria, which are mainly based on lateral shower shape requirements.

Muon candidates are reconstructed using the STACO algorithm. As the relevant muon triggers require combined muon candidates at the EF, only combined muon candidates are considered here, although in the analysis also non-combined muons can be used. A combined muon candidate is constructed using tracks from both the muon spectrometer as well as from the inner detector. Differences between the algorithms used during online and offline reconstruction can induce a non-vanishing probability for offline non-combined muons to have issued a combined muon trigger - the efficiency of a combined muon trigger with respect to non-combined STACO muons has been observed to be considerable in the transition region between the barrel and the endcaps, in particular. Nevertheless, since the fraction of non-combined muons is usually small, there is not enough statistics to measure this efficiency in the required binning for these. Therefore in the analyses the non-combined muons are assigned an efficiency of 0 in MC and are not considered for trigger matching in data. Baseline muons are required to have a transverse momentum of more than $10 \mathrm{GeV}$, lie within $|\eta|<2.4$ and satisfy a selection of hit requirements in the ID. After overlap removal, which is explained in the next paragraph, muons are further required to be isolated; that is, the sum of the transverse momenta of all tracks in a cone of size $\Delta R=0.2$ around the muon track must be less than $1.8 \mathrm{GeV}$.

Overlap removal is performed in five steps using all baseline objects. First, if two baseline electrons are within $\Delta R<0.1$, the electron with the smaller transverse energy is removed. Second, if the distance between any baseline jet and any baseline electron is smaller than $\Delta R<0.2$, the baseline jet is removed. If the distance between any remaining baseline jet and a baseline electron is still less than $\Delta R<0.4$, the electron is removed. In a fourth step all baseline muons that are within a distance of $\Delta R<0.4$ to a remaining baseline jet are removed, and finally both muons and electrons are removed, if they are within a distance of $\Delta R<0.1$ to each other. The reasoning for this procedure is explained in more detail in chapter 6 . 
The efficiency measurements presented in this section have been performed using combined signal muons, i.e. isolated combined muons after overlap removal - these are the muons that in the analyses are used for trigger matching in data and the calculation of trigger weights in MC.

For all measurements that have been used in the analyses, the Tag and Probe method with $Z \rightarrow \mu \mu$ candidates, requiring exactly two muons with $\Delta_{m}=10 \mathrm{GeV}$, has been applied. A background subtraction has not been performed, as the efficiencies are not directly compared to the efficiencies in the $\mathrm{MC}$, but they have been used in several physics analyses to re-weight the MC samples according to the procedure described above.

\section{Measurement of Absolute Efficiencies}

The primary single muon triggers that have been used for the SUSY searches using the data taken in 2011 are EF_mu18 and EF_mu18_medium. The difference between these two trigger items is a tighter L1 criterion for EF_mu18_medium compared to EF_mu18. The item EF_mu18 was used throughout data taking periods A to I in 2011, while due to the increasing trigger rates, the item EF_mu18_medium was used in data taking periods J to M. The absolute efficiency with respect to reconstructed signal muons is shown in Figure 5.13 for EF_mu18 and in Figure 5.14 for EF_mu18_medium. The efficiency is shown as a function of the most relevant muon variables $p_{T}$, and $\eta$ and $\phi$. Note that the one-dimensional histograms in figures 5.13(a) and 5.14(a) show the efficiency as a function of the muon transverse momentum averaged over the $\eta$ and $\phi$ distributions, and that the two-dimensional plots in figures 5.13(b) and 5.14(b) show the efficiency averaged over all muons with a transverse momentum of more than $20 \mathrm{GeV}$, for which the efficiency is roughly flat in $p_{T}$. Also, in the plots showing the efficiency as a function of the transverse momentum, the trigger matching was performed only for muons with $p_{T}>20$ $\mathrm{GeV}$ as explained above. As the difference between the average efficiency in the endcaps and the average efficiency in the barrel region can be large, the efficiency as a function of the transverse momentum is shown separately for these two regions.

In the plateau region, where $p_{T}>20 \mathrm{GeV}$, the average efficiency in the endcaps reaches values between $87 \%$ and $90 \%$ for both triggers, while in the central part of the detector the average efficiencies in this region are about $75 \%$ for EF_mu 18 and about $67 \%$ for EF_mu18_medium. The main reason for the difference in the barrel region is the tightened L1 requirement for the latter trigger item: The EF item EF_mu18 is seeded at L1 by the item L1_MU10, which uses one of the low $p_{T}$ coincidence windows, while EF_mu18_medium is ultimately seeded by L1_MU11, the hypothesis of which includes the high $p_{T}$ coincidence window.

In addition to the single muon triggers, the analysis searching for SUSY signatures in final states with two leptons used a combined electron muon trigger throughout periods $\mathrm{A}$ to $\mathrm{M}$. The EF item EF_e10_medium_mu6 has been used exclusively in some parts of the signal phase space, such that a measurement of the absolute efficiency of the muon trigger item EF_mu6 - for which re-run mode was enabled - has been performed using EF_mu18 or EF_mu18_medium to select the tag muon in the respective data taking periods. The results of this measurement are shown in Figure 5.15, where as for the single muon triggers discussed above no trigger matching for muons in the turn-on region has been performed, such that the efficiency for all muons with $p_{T}<8 \mathrm{GeV}$ is 0 . As for the primary single muon triggers, the efficiency is shown as a function of the muon transverse momentum separately for both the endcaps and the barrel in Figure 5.15(a) and as a function of the pseudorapidity and the azimuthal angle for all muons with $p_{T} \geq 8 \mathrm{GeV}$ in Figure 5.15(b). The results are comparable to the measurements of the efficiency of EF_mu18, because also EF_mu6 is seeded by a L1 trigger that's decision is based on the evaluation of the low- $p_{T}$ coincidence window. 


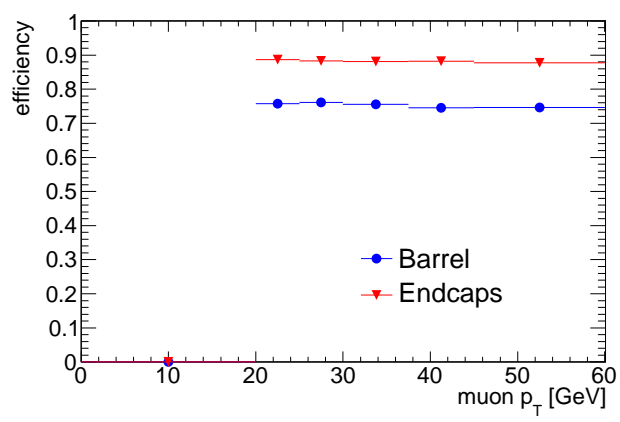

(a)

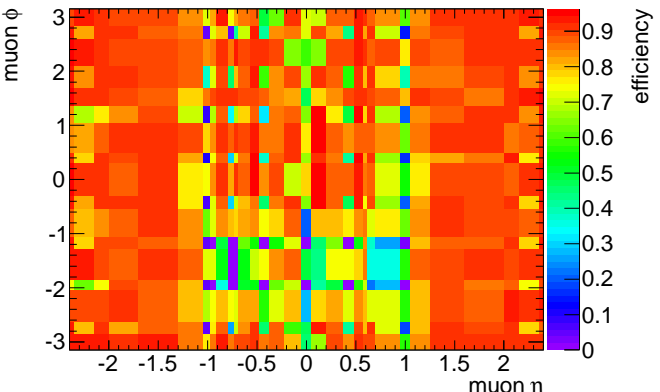

(b)

Figure 5.13: The absolute efficiency with respect to reconstruction of the trigger item EF_mu18. The efficiency is shown as a function of the muon transverse momentum for all muons passing the offline selection cuts in (a), where the barrel region is defined by $|\eta|<1.05$ and the endcap regions are defined by $1.05<|\eta|<2$.4. In (b) the efficiency is shown as a function of the muon $\eta$ and $\phi$ for all muons passing the offline selection cuts and $p_{T}>20 \mathrm{GeV}$. The efficiency has been measured using data taken in 2011 from data taking periods A to I. In the barrel region, for $\phi \sim-1$ and $\phi \sim-2$ the efficiency drops to very low values, which is mainly due to a lack in coverage due to the support structure for the detector that is located in that region. Overall, the efficiency is a highly inhomogeneous function in the barrel region.

\section{Measurement of Conditional Efficiencies}

In order to increase the absolute efficiency of the offline selection, the searches for SUSY with two or more leptons in the final state used symmetric di-muon triggers in 2011. This increased the sensitivity of the searches in parts of the signal phase space where two or more signal leptons have an average transverse momentum of less than $20 \mathrm{GeV}$, because events with such leptons are usually not selected by the single muon triggers.

For the application of the trigger efficiencies in the calculation of event weights for data-MC comparisons, it is not sufficient to calculate the absolute efficiencies of these di-muon triggers. Instead conditional efficiencies need to be measured as explained in the first part of this chapter.

The lowest un-prescaled di-muon trigger that has been available during the complete data taking period in 2011 is EF_2mu10_loose. For the analyses using this trigger the efficiency for the trigger legs EF_mu10_loose had to be measured for muons that fail the trigger hypothesis of the used single muon trigger in the respective data-taking period. This efficiency is referred to as the conditional efficiency of EF_mu10_loose here; it has been calculated for muons not passing EF_mu18 in data taking periods A to I, and for muons not passing EF_mu18_medium in data taking periods J to M. The Tag and Probe method has been used as well, using EF_mu18 in data taking periods $\mathrm{A}$ to $\mathrm{I}$ and EF_mu18_medium in data taking periods $\mathrm{J}$ to $\mathrm{M}$ to select the tag muon. As for EF_mu6 the evaluation of the trigger hypothesis for EF_mu10_loose was done in re-run mode. The conditional efficiency has then been measured using all probe muons that failed the hypothesis of the respective primary single muon trigger. The results are summarised in figures 5.16, and following the usual procedure the actual trigger decision is neglected for $p_{T}<12 \mathrm{GeV}$.

Figures 5.16(a) and 5.16(b) show the conditional efficiencies as a function of the muon $p_{T}$. For $p_{T}<20 \mathrm{GeV}$, the conditional efficiency here is equal to the absolute efficiency of EF_mu10_loose. 


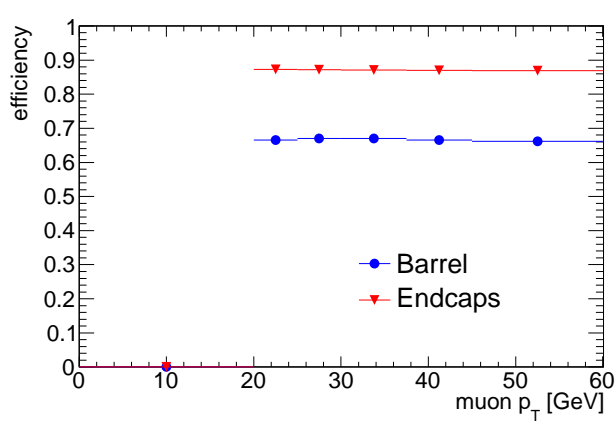

(a)

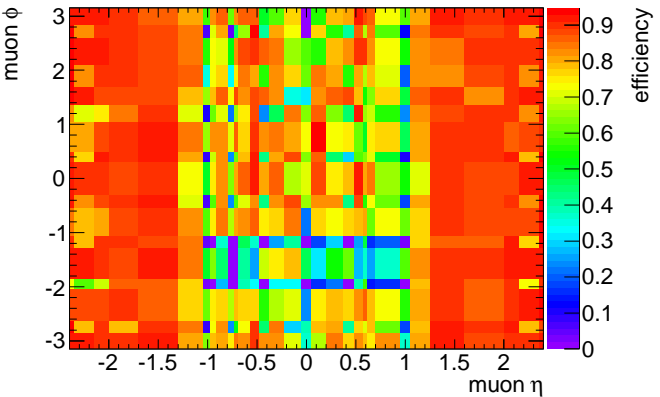

(b)

Figure 5.14: The absolute efficiency with respect to reconstruction of the trigger item EF_mu18_medium as a function of the muon $p_{T}$ in (a) and as a function of $\eta$ and $\phi$ in (b). The efficiency has been measured using data taken in 2011 during periods $\mathrm{J}$ to M. Compared to the performance of EF_mu18 in the previous data taking periods, the efficiency of EF_mu18_medium drops below $70 \%$ in the barrel region.

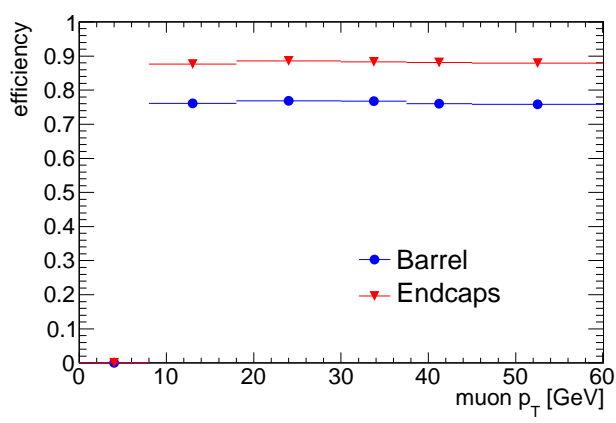

(a)

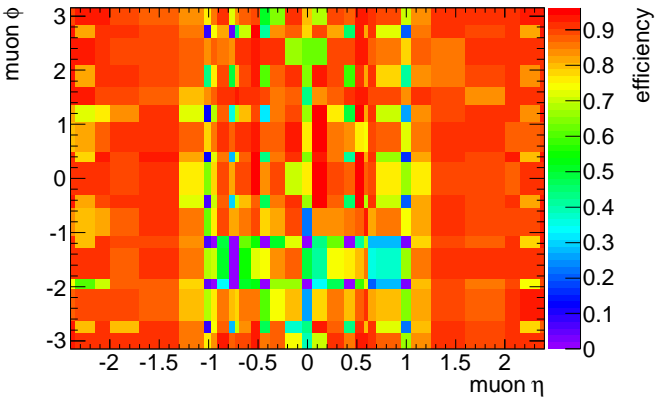

(b)

Figure 5.15: The absolute efficiency with respect to reconstruction of the trigger item EF_mu6 as a function of the muon $p_{T}$ in (a) and as a function of $\eta$ and $\phi$ in (b). The trigger decision was evaluated in re-run mode, the trigger EF_mu18 (_medium) have been used as tag triggers in data taking periods A to I ( $\mathrm{J}$ to $\mathrm{M})$.

Figures 5.16(c) and 5.16(d) show the efficiency of EF_mu10_loose as a function of the muon $\eta$ and $\phi$ in that $p_{T}$ regime. For $p_{T} \geq 20 \mathrm{GeV}$, the efficiency is the actual conditional efficiency, which as a function of $\eta$ and $\phi$ is shown in figures 5.16(e) and 5.16(f). In the one-dimensional plots, the change from the low $p_{T}$ coincidence window to the high $p_{T}$ coincidence window when going from EF_mu18 to EF_mu18_medium is clearly visible in the barrel. The efficiency measurement had to be performed with a much coarser binning compared to the measurements of the absolute efficiencies because the available statistics were much smaller for the measurement of the conditional efficiencies. 


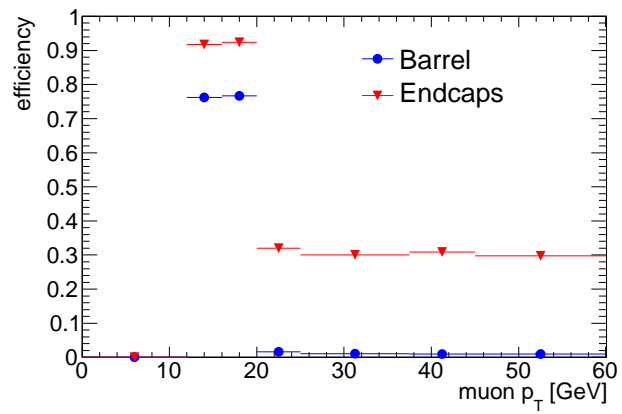

(a)

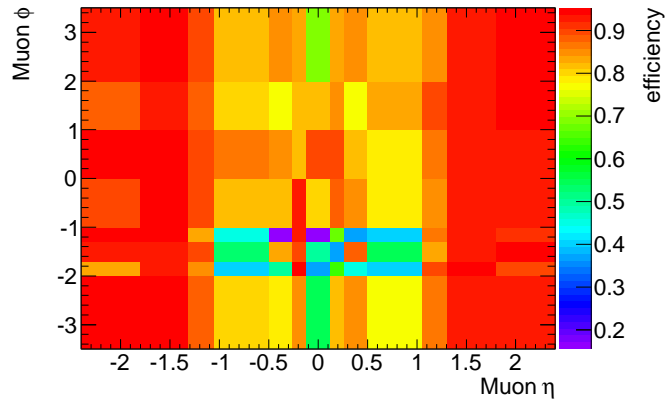

(c)

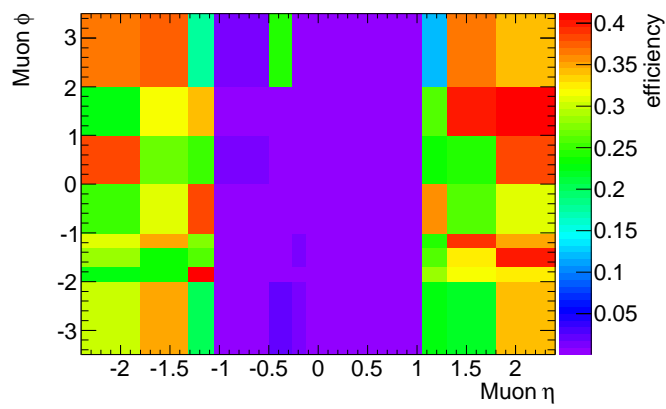

(e)

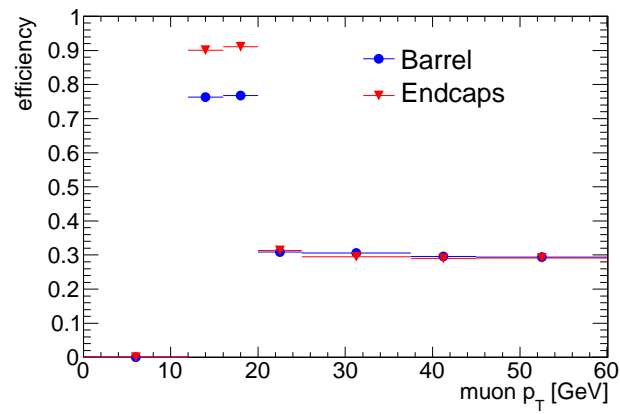

(b)

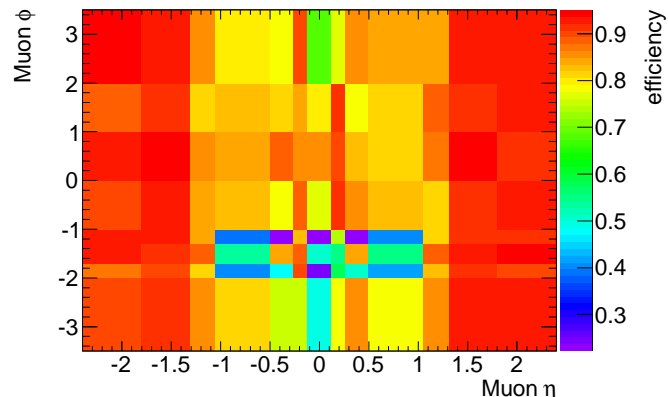

(d)

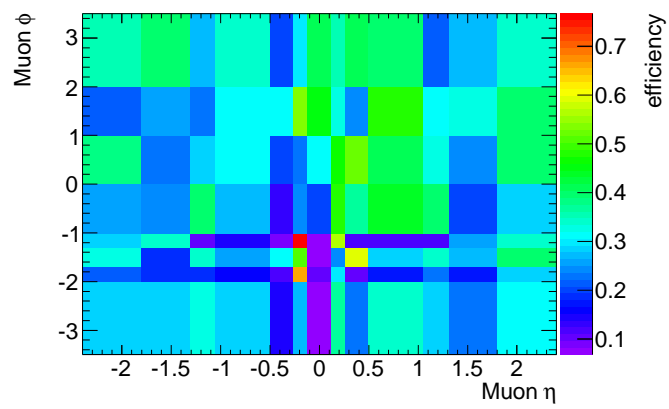

(f)

Figure 5.16: The efficiency of EF_mu10_loose with respect to offline muons that have not passed the trigger EF_mu18 (left side, data taking periods A to I) or the trigger EF_mu18_medium (right side, data taking periods $\mathrm{J}$ to $\mathrm{M}$ ). For $p_{T}>20 \mathrm{GeV}$, in the endcap regions there is a non-vanishing probability that a muon will not pass the single muon triggers, but it will pass the hypothesis of the di-muon trigger EF_2mu10_loose. For EF_mu18_medium, this probability is also considerable in the barrel region. The plots in (c) and (d) show the absolute efficiency for $8 \mathrm{GeV} \leq p_{T}<20 \mathrm{GeV}$ as a function of the muon $\eta$ and $\phi$, while (e) and (f) show the conditional efficiency for $p_{T} \geq 20 \mathrm{GeV}$. Due to the small statistics available for the measurements, a relatively coarse binning has been chosen for these measurements. 



\section{Search for R-Parity Conserving SUSY in Final States with Four Leptons}

While in the more constrained models like the CMSSM searches for supersymmetry with zero or one lepton in the final state are often the most sensitive ones, searches with two or more leptons in the final state become more important if certain assumptions are dropped from these models. If for instance the mass parameters for the coloured sparticles are decoupled from the mass parameters of the colourless sfermions and bosinos, the squarks and gluinos may acquire very large masses at the electroweak scale, while neutralinos, charginos and sleptons remain relatively light, and the direct production of these sparticles may become the dominant production mechanism for supersymmetric particles at the LHC. With similarly tight signal regions, the SM background usually decreases significantly with increasing lepton multiplicity. In the pMSSM, which usually provides the framework for motivating searches with two or more leptons, the direct production of gauginos is one of the processes probed at the LHC, cf. Figure 2.6. Since in the pMSSM it is often a single production process that would drive an observation of physics beyond the SM at one point in the pMSSM parameter space, so-called simplified models are constructed and studied for the direct production of gauginos and sleptons. Searches for SUSY with two leptons in the final state usually target the direct production of charginos and sleptons, whereas a search with three leptons in the final state would primarily be sensitive to the associate production of a chargino and a neutralino. A promising candidate for a discovery in final states with four leptons is the associate production of two neutralinos, which is studied in this chapter.

A study of the pMSSM is first presented as a motivation for the construction of a simplified model for the associate production of two neutralinos. Two slightly different simplified models are introduced. After this, a search for SUSY with four leptons in the final state with the ATLAS detector, using the full dataset of 2012 is presented, and the results are interpreted in the simplified models. The work that is presented in this chapter is a part of a more comprehensive analysis, which is documented in [147].

\subsection{Simplified Models for $\tilde{\chi}_{2}^{0}-\tilde{\chi}_{3}^{0}$ Production}

Two slighlty different simplified models for the associate production of a $\tilde{\chi}_{2}^{0}$ and a $\tilde{\chi}_{3}^{0}$ with four leptons in the final state have been defined. In both models, the abstract mass hierarchy is the same, $m_{\tilde{l}_{L}}>m_{\tilde{\chi}_{2 / 3}^{0}}>m_{\tilde{l}_{R}}>m_{\tilde{\chi}_{1}^{0}}$, and in both models the produced neutralinos decay exclusively into a right chiral slepton and a charged lepton, and the right chiral sleptons decay exclusively into a charged lepton and the LSP. In one of the models, the mass splittings between the neutralinos are fixed, and the model is parametrised in terms of the absolute neutralino mass scale and the mass difference between the neutralinos and the right chiral sleptons. In the second model, the mass splitting between the heavier neutralinos and the LSP is varied, as well as the absolute neutralino mass scale, and the right chiral slepton masses are fixed in between the masses of the $\tilde{\chi}_{2}^{0}$ and the $\tilde{\chi}_{1}^{0}$. 
The pMSSM has been used as a starting point to define the raw structure of the SUSY mass spectrum and the relevant couplings in the simplified models. The study of the pMSSM from which the baseline properties of the simplified models have been derived is presented first, followed by a more complete description of the two simplified models.

\subsubsection{The pMSSM as a Motivation}

In the case of heavy, coloured superpartners the dominant production mechanism at the LHC will be the direct electroweak production of sleptons, neutralinos and charginos, if the masses of those are small enough. Also, since the squarks are heavy, the only available decay modes for the heavier neutralinos and charginos are the decays via sleptons, the electroweak gauge bosons and the Higgs bosons. In these scenarios it is the masses of the sleptons and the mixings of the neutralinos and charginos that will determine the relative branching fractions of each of the following two-body and three-body decays, all assuming a neutralino LSP:

$$
\begin{aligned}
& \tilde{\chi}_{i}^{0} \rightarrow \tilde{l}_{R} l \\
& \tilde{\chi}_{i}^{0} \rightarrow \tilde{\chi}_{j}^{0} h / H / A \\
& \tilde{\chi}_{i}^{ \pm} \rightarrow \tilde{\nu}_{l} l \\
& \tilde{\chi}_{i}^{ \pm} \rightarrow \tilde{\chi}_{j}^{0} f f
\end{aligned}
$$

$$
\begin{aligned}
& \tilde{\chi}_{i}^{0} \rightarrow \tilde{l}_{L} l \\
& \tilde{\chi}_{i}^{0} \rightarrow \tilde{\chi}_{i}^{0} f f \\
& \tilde{\chi}_{i}^{ \pm} \rightarrow \tilde{\chi}_{j}^{0} W^{ \pm} \\
& \tilde{l}_{L / R} \rightarrow \tilde{\chi}_{j}^{0} l
\end{aligned}
$$

$$
\begin{aligned}
& \tilde{\chi}_{i}^{0} \rightarrow \tilde{\chi}_{j}^{0} Z \\
& \tilde{\chi}_{i}^{ \pm} \rightarrow \tilde{l}_{L} \nu_{l} \\
& \tilde{\chi}_{i}^{ \pm} \rightarrow \tilde{\chi}_{j}^{0} H^{ \pm} \\
& \tilde{l}_{L / R} \rightarrow \tilde{\chi}_{j}^{ \pm} \nu_{l}
\end{aligned}
$$

where $f$ is an arbitrary SM fermion. Therefore, in the pMSSM with heavy coloured sparticles, the pair production of neutralinos is one of the few possibilities to get final states with four leptons.

At the LHC, the production of a neutralino in association with a chargino is usually favoured with respect to the associate production of two neutralinos. This is because the dominant production mechanism for a neutralino-chargino pair is the exchange of a $W$ boson in the schannel, whereas the associate production of two neutralinos requires an intermediate $Z$ boson. In order to identify regions of the parameter space of the pMSSM where a search with four leptons in the final state is potentially more sensitive than a search with three leptons in the final state an analysis of the behaviour of the production cross sections and branching fractions of the relevant processes has been performed.

In principle it is also possible to get a final state with two or less leptons for the points that are considered in this analysis. These processes are only of marginal interest here; the cross section times branching fraction for final states with two or less leptons is indeed usually larger than those for final states with three and more leptons - due to the similar production mechanism not by a larger factor - but with similar acceptance cuts the expected SM background is much larger in these channels.

A scan of a slice of the pMSSM parameter space has been conducted with the goal of identifying regions in the pMSSM parameterspace where two criteria are met: a) the cross section for direct gaugino production is large enough to allow for a discovery at the LHC, and b) the ratio

$$
\mathcal{R}=\frac{\sigma\left(p p \rightarrow \tilde{\chi}_{i}^{0} \tilde{\chi}_{j}^{0}\right) \times B R\left(\tilde{\chi}_{i}^{0} \rightarrow l l X\right) \times B R\left(\tilde{\chi}_{j}^{0} \rightarrow l l X\right)}{\sigma\left(p p \rightarrow \tilde{\chi}_{i}^{0} \tilde{\chi}_{k}^{ \pm}\right) \times B R\left(\tilde{\chi}_{i}^{0} \rightarrow l l X\right) \times B R\left(\tilde{\chi}_{k}^{ \pm} \rightarrow l X\right)},
$$

is large enough such that a search with four leptons in the final state is more sensitive than a search with three leptons in the final state. In order to identify such regions, the total production cross section for neutralino pairs and the branching fraction of final states with four leptons has been calculated at each point in the scan and the ratio $\mathcal{R}$ has been examined at these points. For the calculation of $\mathcal{R}$, the values $i, j=\{2,3\}$ and $k=1$ have been taken into account. The 
reasons for not considering the heaviest neutralino, i.e. $i, j=4$ or the heaviest chargino, i.e. $k=2$, is that the production of these is highly suppressed with respect to the lighter states due to their relatively large masses. It is also possible to produce the lightest neutralino directly, in particular if this is Higgsino-like. The direct production of a $\tilde{\chi}_{1}^{0}$ in association with a second, Higgsino-like neutralino does not contribute to the inclusive four lepton production cross section that is of interest here; furthermore, Higgsino-like neutralino LSPs are disfavoured by direct cold dark matter detection experiments.

For this study of the pMSSM, the coloured sector has been completely decoupled by setting the mass parameters for the squarks and the gluinos to $10 \mathrm{TeV}$, which yields physical masses above the kinematic thresholds at the $8 \mathrm{TeV}$ LHC. The masses and mixings of the neutralinos and charginos are mainly determined by the parameters $M_{1}, M_{2}$ and $\mu$, which have been varied between $50 \mathrm{GeV}$ and $1000 \mathrm{GeV}\left(M_{1}\right.$ and $\left.M_{2}\right)$ and between $50 \mathrm{GeV}$ and $400 \mathrm{GeV}(\mu)$ in steps of 50 $\mathrm{GeV}$ each. For values larger than the upper boundaries, the sparticles acquire large masses and the production cross sections decrease such that at the LHC with a centre-of-mass energy of 8 $\mathrm{TeV}$ there is no possibility of finding any evidence for the existence of these particles. Commonly a $\mu$ parameter above $\sim 400 \mathrm{GeV}$ is not considered, as larger values usually render a model unnatural. The value of $400 \mathrm{GeV}$ is not a harsh upper bound, and as it was discussed above, strictly speaking the naturalness criterion does not constitute a physical boundary. Nevertheless, as for the study presented here the consideration of $\mu>400 \mathrm{GeV}$ is not crucial, the scan has been performed up to this value only.

In correspondence to the CMSSM, the mass parameters for the left chiral sleptons have been set to larger values than those for the right chiral sleptons for most values of $M_{1}, M_{2}$ and $\mu$, i.e. $m_{\tilde{l}_{L}}=0.8 \cdot\left(M_{1}+M_{2}\right)$ and $m_{\tilde{l}_{R}}=0.25 \cdot\left(M_{1}+M_{2}+\mu\right)$. The choice for $m_{\tilde{l}_{R}}$ ensures that at most points tested the right chiral sleptons are heavier than the lightest neutralino, but lighter than the heavier neutralinos; however, this is not true for all points, as will be shown later. Also, the simplified models are designed for use in a search for SUSY with four leptons excluding $\tau$ 's in the final state, such that also for the staus the mass parameters have been set to relatively large values, i.e. $m_{\tilde{\tau}_{i}}=m_{\tilde{l}_{L}}$. The choice made for the mass parameters of the left chiral sleptons and staus also decouples the left chiral sleptons from the interesting processes, while still keeping the mass difference between the left chiral and right chiral sleptons relatively small.

Finally the trilinear couplings have been fixed to $A_{t}=-3 \mathrm{TeV}, A_{\tau}=A_{b}=0$, and the Higgs mass parameter $m_{A}$ has been fixed to $1.5 \mathrm{TeV}$. This ensures that the mass of the lightest Higgs boson is given by $120-130 \mathrm{GeV}$, roughly, at all points. Different values of $\tan \beta$ have been taken into account - the results for $\tan \beta=10$ are shown, as these are the only results relevant for the two simplified models that are presented here.

For the points under investigation, the production cross section times branching fraction for final states with three and four leptons have been calculated using the program SoftSUSY [96] as a spectrum calculator, the program SDECAY [148] for the branching fractions and the program prospino [149] for the production cross section at the LHC with a centre-of-mass energy of 8 $\mathrm{TeV}$. All the decay modes listed above are taken into account, and the production processes

$$
\begin{aligned}
& p p \rightarrow \tilde{\chi}_{i}^{0} \tilde{\chi}_{j}^{0} \\
& p p \rightarrow \tilde{\chi}_{i}^{0} \tilde{\chi}_{k}^{ \pm}
\end{aligned}
$$

are considered. Apart from the mass scale, it is the mixing matrices that determine the production cross sections for the electroweak gauginos. The direct production of sleptons is not taken into account - the left chiral sleptons typically have a large muss in the chosen scenarios, such that the production cross section is very small. The direct production of right chiral sleptons 

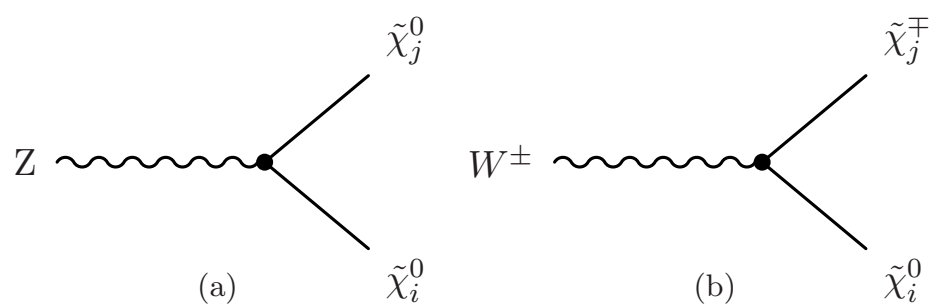

Figure 6.1: The dominant vertices for the direct production of a neutralino in association with another gaugino at the LHC. The production via a Higgs boson is usually negligible.

also has a small cross section and does neither contribute to the three lepton final states, nor to the four lepton final states at the points that are of relevance for this analysis.

The dominant vertices for the most relevant production processes are shown in Figure 6.1. With the mixing matrices $N, U$, and $V$ as defined in chapter 2 the corresponding vertex factors are given by:

$$
\begin{aligned}
& \text { (a) : } \frac{i e}{2 \sin \theta_{W} \cos \theta_{W}} \gamma^{\mu}\left(\left(N_{4 i}^{*} N_{4 j}-N_{3 i}^{*} N_{3 j}\right) P_{L}-\left(N_{4 i} N_{4 j}^{*}-N_{3 i} N_{3 j}^{*}\right) P_{R}\right) \\
& \text { (b) : } \frac{i e}{2 \sin \theta_{W} \cos \theta_{W}} \gamma^{\mu}\left(\left(N_{2 i} V_{1 j}^{*}-\frac{1}{\sqrt{2}} N_{4 i} V_{2 j}^{*}\right) P_{L}+\left(N_{2 i}^{*} U_{1 j}+\frac{1}{\sqrt{2}} N_{3 i}^{*} U_{2 j}\right) P_{R}\right)(6
\end{aligned}
$$

From (a) it is clear that only the Higgsino components of a neutralino contribute to the associate production of two neutralinos via a $Z$ boson. This is because neither the Bino nor the Wino do carry a weak hypercharge, and therefore do not couple to the $B$; also due to the SU (2) structure constants, the neutral Wino does not couple to the $W^{0}$. It is also clear that in the absence of complex phases in the mixing matrix, for $i=j$ the production of a neutralino pair is highly suppressed if the absolute values of the Higgsino components of that neutralino are roughly equal, i.e. $\left|N_{i 3}\right| \approx\left|N_{i 4}\right|$. The associate production of two Higgsino-like neutralinos with $i \neq j$ on the other hand can become large if the relative signs of the $H_{u}$ or the $H_{d}$ component are opposite, as in this case these two mass eigenstates behave like a Dirac fermion pair. It is possible to directly produce Binos and neutral Winos via the exchange of a Higgs boson in the s-channel; this process is however highly suppressed in comparison with the production via an electroweak gauge boson.

The associate production of a neutralino and a chargino happens via an intermediate $W$ boson. The Wino components of both gauginos contribute as well as the Higgsino components. For a fully Bino-like neutralino, the vertex factor is zero.

Figure 6.3 shows the production cross section for the associate production of two neutralinos, $\sigma\left(p p \rightarrow \tilde{\chi}_{i}^{0} \tilde{\chi}_{j}^{0}\right)$ with $i, j=\{1,2\}$, in leading order in the $M_{2}-\mu$ plane for different values of $M_{1}$. Points that have been assigned a number are excluded because at these points the stable LSP is one of the sleptons $(-1)$ or because they are excluded by theory $(-2$, due to non-convergent RGE, tachyons, no radiative EWSB). For all values of $M_{1}$, there are regions in the $M_{2}-\mu$ plane with a considerably large production cross section for neutralino pairs. Depending on $M_{1}$, the behaviour of the cross sections is qualitatively different as a function of $M_{2}$ and $\mu$ and there is a number of different reasons for these behaviours, such that a general statement is difficult to make. Based on the examples shown in the figure, the behaviour is now discussed rudimentary 


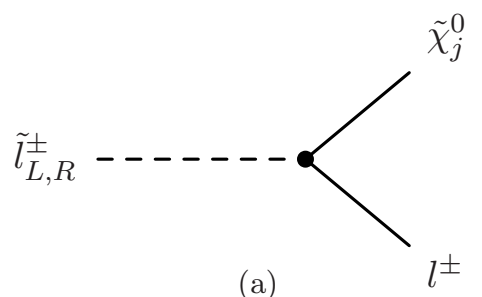

(a)

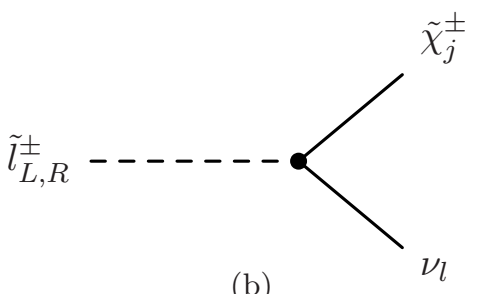

(b)

Figure 6.2: The relevant vertices for the decay of the right and left chiral sleptons. Due to the small Yukawa couplings in the first and second generation of leptons, the decay into a chargino and a neutrino is usually heavily suppressed for the right chiral sleptons.

for the more relevant regions of the pMSSM parameter space. As discussed above, the most important ingredients for the production cross section are the masses of the involved particles as well as the neutralino mixing matrix.

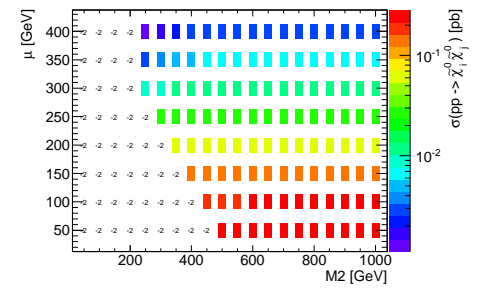

(a) $M_{1}=100 \mathrm{GeV}$

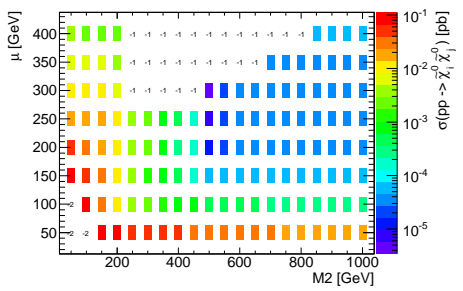

(d) $M_{1}=550 \mathrm{GeV}$

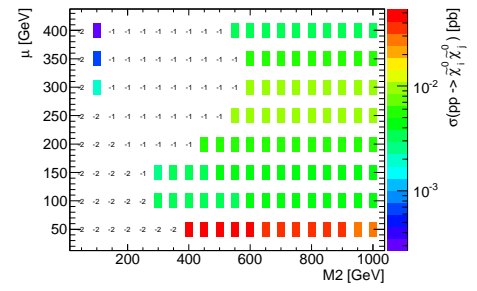

(b) $M_{1}=250 \mathrm{GeV}$

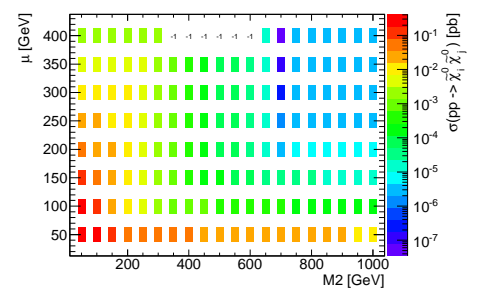

(e) $M_{1}=750 \mathrm{GeV}$

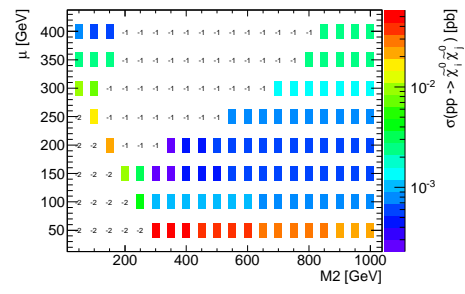

(c) $M_{1}=350 \mathrm{GeV}$

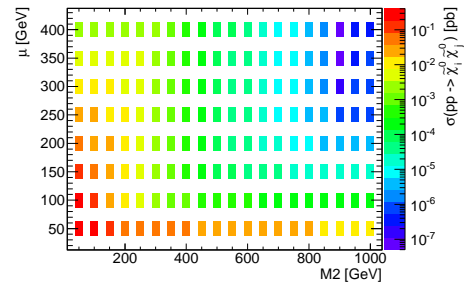

(f) $M_{1}=950 \mathrm{GeV}$

Figure 6.3: The production cross section for a pair of neutralinos at $\sqrt{s}=8 \mathrm{TeV}$ at the LHC as a function of $M_{2}$ and $\mu$ for different values of $M_{1}$. The lightest and the heaviest neutralino have not been considered, such that $i, j=\{2,3\}$, here.

- $M_{1}=100 \mathrm{GeV}$, Figure 6.3(a): The dominant contribution to the neutralino pair production cross section comes from the production of a $\tilde{\chi}_{2}^{0}$ in association with a $\tilde{\chi}_{3}^{0}$. The production of a $\tilde{\chi}_{2,3}^{0}$ pair does not contribute significantly. With the exception of a few points with $M_{2} \lesssim 400 \mathrm{GeV}$ and $\mu \gtrsim 300 \mathrm{GeV}$, where it becomes more Higgsino-like, the $\tilde{\chi}_{4}^{0}$ is almost exclusively a Wino; this means that the Wino components of the other neutralinos are negligible. For $\mu \gtrsim 150 \mathrm{GeV}$, the Bino component of the $\tilde{\chi}_{1}^{0}$ is close to 1 , which means that in this region the $\tilde{\chi}_{2,3}^{0}$ have very large Higgsino components; the cross section decreases with increasing $\mu$ due to the larger masses of the Higgsino-like neutralinos in that case. The relative sign of the two neutralino components does not play a major role, here. 
- $M 1=250 \mathrm{GeV}$, Figure 6.3(b): The dominant production mechanism is again the associate production of a $\tilde{\chi}_{2}^{0}$ and a $\tilde{\chi}_{3}^{0}$, with the exception of all points with $\mu=50 \mathrm{GeV}$. For these values, the pair production of two $\tilde{\chi}_{0}^{2}$ is dominant - usually this is larger by a factor of about 10 compared to the associate production; the pair production of two $\tilde{\chi}_{3}^{0}$ is negligible. There is a significant drop in the production cross section for a $\tilde{\chi}_{2}^{0}$ pair between $\mu=50$ $\mathrm{GeV}$ and $\mu=100 \mathrm{GeV}$, such that already at $\mu=100 \mathrm{GeV}$ the associate production is dominant. The reason for this is that (a) the mass of the second lightest neutralino gets larger, and more importantly (b), the difference of the absolute values of the two Higgsino components of that neutralino become smaller, which results in a much smaller vertex factor as described above. For increasing values of $\mu$, the associate production becomes the more dominant, the larger $\mu$ gets. Apart from the small stripe at $M_{2}=100 \mathrm{GeV}$, where the overall production cross section is very small, the heaviest neutralino is again mostly Wino. For $\mu<250 \mathrm{GeV}$, the $\tilde{\chi}_{3}^{0}$ has a large Bino component, which explains the small cross section for the associate production in this region. For larger values of $\mu$, the $\tilde{\chi}_{3}^{0}$ gets more Higgsino-like and the Bino component is mostly absorbed in the lightest neutralino, such that the associate production of a $\tilde{\chi}_{2}^{0}$ and a $\tilde{\chi}_{3}^{0}$ is enhanced with increasing $\mu$; this effect is countervailed by the increasing masses, such that the cross section for the associate production drops again at the upper edge of the tested $\mu$ spectrum.

- $M_{1}=350 \mathrm{GeV}$, Figure 6.3(c): This example is used here to describe some features that are common to most of the points with $250 \mathrm{GeV} \lesssim M_{1} \lesssim 400 \mathrm{GeV}$ : The composition of the heaviest neutralino changes rapidly as a function of $M_{2}$ in a very small interval in the relevant region of the $M_{2}-\mu$ plane. This behaviour is observed for all such values of $M_{1}$ where small values of $M_{2}$ are no longer excluded by theory, but it becomes more pronounced for larger values of $M_{1}$. For all values of $M_{1}>300 \mathrm{GeV}$, the Wino component of the heaviest neutralino is close to 0 for $M_{2} \lesssim M_{1}-100 \mathrm{GeV}$ and the $\tilde{\chi}_{4}^{0}$ becomes mostly a Bino for all values of $\mu$ that have been considered here; in that case, for smaller values of $\mu$, the Wino component is absorbed by the $\tilde{\chi}_{3}^{0}$, while for larger values of $\mu$ and small values of $M_{2}$ the Wino component is absorbed in the lightest neutralino. For $M_{2} \gtrsim M_{1}$ however, the Wino component of the heaviest neutralino gets close to 1 . For $\mu \lesssim M_{1}-50 \mathrm{GeV}$ and large values of $M_{2}$, the $\tilde{\chi}_{3}^{0}$ is almost exclusively a Bino, while the $\tilde{\chi}_{2}^{0}$ and the $\tilde{\chi}_{1}^{0}$ are Higgsinolike. For $\mu \gtrsim M_{1}-50 \mathrm{GeV}$, the Bino-component of the lightest neutralino becomes larger, such that in this region the second and third lightest neutralinos are Higgsino-like, and the production cross section for the associate production becomes larger. The observed behaviour of the cross section for $\mu=50 \mathrm{GeV}$ can be explained in the same way as it was done for $M_{1}=250 \mathrm{GeV}$ : In this region, the pair production of two $\tilde{\chi}_{2}^{0}$ dominates.

The behaviour in the remaining examples shown in Figure 6.3 is not explained here in detail. The principle as it has been discussed in some detail above is always the same, and in the following it will be shown that the examples with $M_{1}>350 \mathrm{GeV}$ are not too interesting for the construction of the simplified models that this study aims at. The goal of the following considerations has been to further reduce the region of the pMSSM parameter space that is potentially interesting for a search with four leptons in the final state.

In order to do so, the branching fractions of final states with four leptons have been included in the considerations. The decays of the neutralinos and sleptons are also governed by the mixing matrices. First, charginos and neutralinos can decay via the electroweak gauge bosons. In these cases, the vertex factors (6.3) apply. Second, charginos and neutralinos can decay via a slepton, and sleptons can decay into a lepton and a lighter chargino/neutralino. The vertices most relevant for the study presented here are shown in Figure 6.2. The full vertex factors for 
these diagrams are provided in [48]; they contain the slepton and neutralino/chargino mixing matrices and are not given here, but the most relevant features of the couplings between sleptons and gauginos are briefly summarised.

The coupling strength between neutralinos and sleptons is dominantly driven by the Bino and Wino components of the neutralinos. This is because these components interact with the sleptons with the respective gauge coupling strength, while the interaction between the Higgsino components and the sleptons is described by the Yukawa couplings. Compared to the gauge couplings, for the first two generations the Yukawa couplings are negligible, if the Bino/Wino component of the neutralino in question is not too small ( $\gtrsim 10^{-3}$, very roughly). Also it is only the Bino component of a neutralino that couples to the right chiral sleptons, whereas both the Wino and the Bino component interact with the left chiral sleptons.

The same is essentially true for charginos, with the exception that a chargino does not have a Bino component. This means that for the first two generations, due to the small Yukawa couplings, the decay of a chargino into a right chiral slepton and a neutrino is highly suppressed with respect to the decay via a left chiral slepton, or both the two-body as well as the three-body decay via a SM gauge boson; this holds even if the Wino component of the chargino is relatively small. Furthermore the decay of a right chiral slepton into a chargino and a neutrino is highly suppressed with respect to the decay of the same sparticle into a neutralino and a charged lepton if that decay is kinematically allowed. As an example, some results are shown in Figure 6.4. For the different values of $M_{1}$, two different regions with a relatively large four lepton cross section, which is mainly driven by the mixing of the neutralinos and the mass difference between the neutralinos and the sleptons, emerge:

- For smaller values of $M_{1}$ points with $\mu \sim M_{1} \pm 50 \mathrm{GeV}$ for $M_{2} \sim(2.5 \pm 0.5) \cdot \mu$ very roughly come with a large four lepton cross section.

- For larger values of $M_{1}$, the region with small $M_{2}$ and $\mu$ is no longer theoretically excluded for the chosen slepton mass parameters, such that for these points there is a narrow diagonal in the $M_{2}-\mu$ plane with a relatively large neutralino pair production cross section at $M_{2} \sim \mu$ for $\mu \sim 0.5 \cdot M_{1}$.

- For very large values of $M_{1}$, the cross section times branching ratio for very small values of $\mu$ or $M_{2}$ gets equally large.

For small values of $\mu$, the large cross section for the pair production of two $\tilde{\chi}_{2}^{0}$ over the whole $M_{1}$ and $M_{2}$ plane is multiplied by a very small branching fraction for a four lepton final state and therefore rendered uninteresting for a four lepton search; typically, the expected number of SUSY events with four leptons in the final state in these regions of the pMSSM is at the order of 1 for an integrated luminosity of $10-30 \mathrm{fb}^{-1}$ - without accounting for a limited acceptance and selection efficiency. The reason for the expected number of events with four leptons being this small is that at these points the mass of second lightest neutralino is very small and the right chiral sleptons are much heavier. The only allowed two-body decay of the $\tilde{\chi}_{2}^{0}$ is the decay into the LSP and a photon - this has usually a very small branching fraction, such that the threebody decays are dominating. Since it is mostly a Higgsino, the decay via an off-shell massive gauge boson is most important; either via an off-shell $Z$ boson into the LSP and a fermion pair, or via an off-shell $W$ boson into the light chargino (that will decay further) and a fermion pair, where the probability that in the final state there is a pair of charged leptons is usually at the order of $5 \%$; thus, considering both legs, the probability to get a final state with four charged leptons in these cases is less than $1 \%$. 


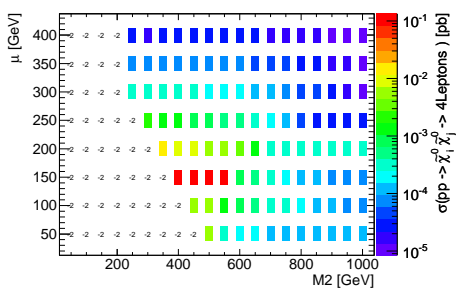

(a) $M_{1}=100 \mathrm{GeV}$

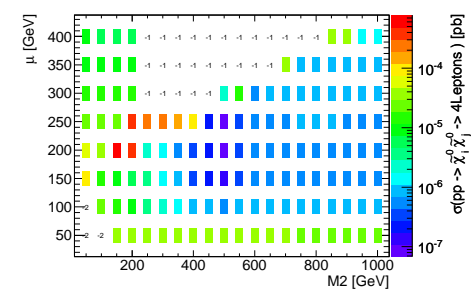

(d) $M_{1}=550 \mathrm{GeV}$

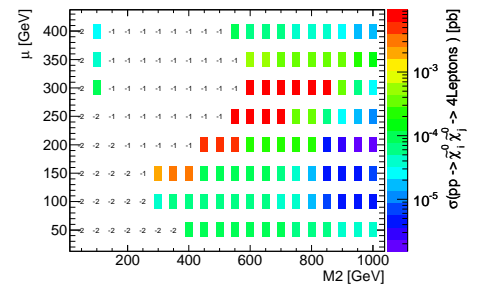

(b) $M_{1}=250 \mathrm{GeV}$

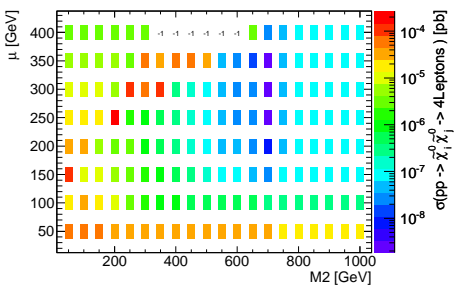

(e) $M_{1}=750 \mathrm{GeV}$

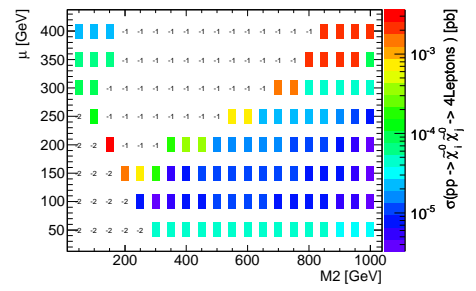

(c) $M_{1}=350 \mathrm{GeV}$

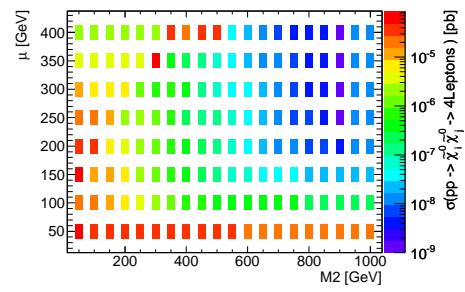

(f) $M_{1}=950 \mathrm{GeV}$

Figure 6.4: The production cross section for a pair of neutralinos times the branching fraction into four lepton final states as a function of $M_{2}$ and $\mu$ for different values of $M_{1}$. A large number of possible decay modes contributes to the branching fraction - in the parts of the parameter space with a large four lepton cross section, the decay of both the $\tilde{\chi}_{2}^{0}$ and the $\tilde{\chi}_{3}^{0}$ into a right chiral slepton and a lepton dominate.

Therefore the dominating process for points where a four lepton search is of potential interest is the associate production of a Higgsino-like $\tilde{\chi}_{2}^{0}$ and a Higgsino-like $\tilde{\chi}_{3}^{0}$. Anticipating a dataset of the order of $10-30 \mathrm{fb}^{-1}$, only points with $\sigma\left(p p \rightarrow \tilde{\chi}_{i}^{0} \tilde{\chi}_{j}^{0} \rightarrow 4 l+X\right) \gtrsim 5 \cdot 10^{-4} \mathrm{pb}$ were considered as a possible benchmark for a simplified model, whereas points with a smaller cross section times branching ratio have no relevance for this study. The plots shown in Figure 6.4 indicate that this threshold for any considered value of $M_{2}$ and $\mu$ is reached at about $M_{1} \sim 550$ $\mathrm{GeV}$.

While for larger values of $M_{1}$ the absolute production cross section can still be large, at the order of $10 \mathrm{fb}$ or even above, in particular for small values of $M_{2}$, one of the branching fractions for $\tilde{\chi}_{2,3}^{0} \rightarrow l l X$ is small:

- If, on the one hand, the decays of the $\tilde{\chi}_{2}^{0}$ are the limiting factor, this is mostly because the masses of the sleptons are larger than the mass of the $\tilde{\chi}_{2}^{0}$, and the same reasoning as for $\mu \sim 50 \mathrm{GeV}$ applies.

- On the other hand, there are still points where the $\tilde{\chi}_{2}^{0}$ decays into two leptons and an LSP via the right chiral sleptons in nearly $100 \%$ of all cases - for nearly all of these points, the decay of the second lightest neutralino into the LSP and a $Z$ boson or into a chargino and a $W$ boson are forbidden by energy-momentum conservation; the mass splittings between the sparticles are too small, here. For these points it is the decay of the $\tilde{\chi}_{3}^{0}$ that limits the four lepton cross section, and for most points it is the decay of this neutralino into a chargino and a $W$ boson that dominates. The decay via the right chiral sleptons is still possible but highly suppressed at these points, because only the Bino component of the $\tilde{\chi}_{3}^{0}$ contributes to that decay and this component of the $\tilde{\chi}_{3}^{0}$ is very small. The decay into 
a $Z$ boson and the LSP is also heavily suppressed because the signs of the two Higgsino components are the same for both the $\tilde{\chi}_{3}^{0}$ and the $\tilde{\chi}_{1}^{0}$. Instead the $\tilde{\chi}_{3}^{0}$ has a considerable Wino component, such that the decay into a chargino and a $W$ boson is highly favoured, and the probability to get four leptons in the final state becomes small due to the branching fractions of the leptonic $W$ and chargino decays: For nearly all points considered in this study, the decay of the light chargino into a slepton and a neutrino are small, because (a) the left chiral sleptons are heavier than the light chargino, and (b) the decay of a chargino into a right chiral slepton is proportional to the respective Yukawa coupling, which is very small for the first two generations. Therefore it is usually the decay of a chargino into a $W$ boson and the LSP that is highly favoured.

For $M_{1} \lesssim 500 \mathrm{GeV}$, there are points in the $M_{1}-\mu$ plane for which the four lepton cross section is large enough, such that the experiments at the LHC are sensitive to these points. It is the same principles as outlined above that govern the behaviour of the cross section times branching fraction. The points with the largest four lepton cross section naturally have a branching ratio of $100 \%$ for the decay of both the $\tilde{\chi}_{2}^{0}$ and the $\tilde{\chi}_{3}^{0}$ into a slepton and a lepton, such that both neutralinos that are produced ultimately feature a final state with two leptons.

However, there are a lot of points with $M_{1} \lesssim 500 \mathrm{GeV}$ and a four lepton cross section of more than $5 \cdot 10^{-4} \mathrm{pb}$ where other decay channels are open. One important effect in this region is the decay of the $\tilde{\chi}_{3}^{0}$ into a $Z$ boson and an LSP. Starting at the maximum four lepton cross section for each value of $M_{1}$, this decay becomes kinematically accessible for increasing values of $\mu$, and is favoured with respect to the sleptonic decays due to the large Higgsino components of the $\tilde{\chi}_{3}^{0}$. Another important effect is that - again, starting at the maximum four lepton cross section at each value of $M_{1}$ - for decreasing values of $\mu$, the right chiral sleptons become heavier than the $\tilde{\chi}_{2}^{0}$, such that decays via the off-shell gauge bosons are preferred again. Finally, for increasing values of $M_{2}$, both of the above mentioned effects kick in.

One of the guiding principles for the construction of a simplified model is to keep the complexity of that model at a minimum, and the consideration of all the above described features in the simplified model does not serve that purpose. Also, while all the points with a four lepton cross section of more than $5 \cdot 10^{-4} \mathrm{pb}$ could possibly be discovered with the available dataset, other channels might be more sensitive.

Therefore, in a final step, the ratio $\mathcal{R}$ as defined in equation (6.1) is shown in Figure 6.5, where all direct gaugino production processes and decays as listed above have been taken into account. Note that in contrast to figures 6.3 and 6.4 the plots are shown for a different selection of values of $M_{1}$ : First, points with $M_{1}>550 \mathrm{GeV}$ are no longer considered due to the small absolute production cross section. Second, in the following also points with $M_{1}>400 \mathrm{GeV}$ are not considered, because for these values of $M_{1}$, in the regions with a large four lepton production cross section the ratio $\mathcal{R}$ is small, such that a search with three leptons in the final state seems more promising, and in the regions where $\mathcal{R}$ is relatively large, the four lepton production cross section is too small to allow for a potential discovery.

In the following consideration the selection efficiency for a four lepton selection is assumed to be roughly equal to the selection efficiency of a three lepton selection - a more accurate calculation requires the use of a detailed MC simulation, which has not been available at this point; after all, this study has been performed to design the parameters for a MC production. The assumption is justified due to the similarities in the production and decay of the relevant particles for a three lepton final state and a four lepton final state. The analyses that were performed by the ATLAS Collaboration before this study has been done indicate that very roughly, for similar production processes, the SM background in a search with three leptons in the final state is about a factor of $12-25$ larger than the SM background in a search with a four 
lepton final state $[150,151]$. In that case, as a very rough estimate again, a four lepton search is more sensitive than a three lepton search, if $\mathcal{R} \gtrsim 0.4-0.5$ - assuming a (roughly realistic) signal over background ratio of $\sim 3$ in the four lepton channel. The plots shown in Figure 6.5 indicate that this can only be achieved for $100 \mathrm{GeV} \leq M_{1} \leq 350 \mathrm{GeV}$, such that also points with $M_{1}<100 \mathrm{GeV}$ or $M_{1}>350 \mathrm{GeV}$ are no longer taken into account for this study.

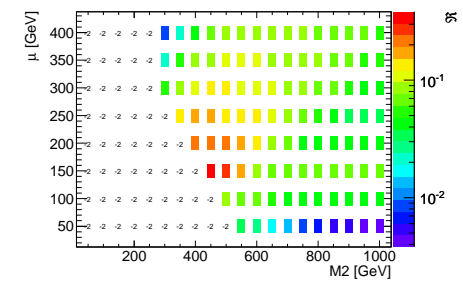

(a) $M_{1}=50 \mathrm{GeV}$

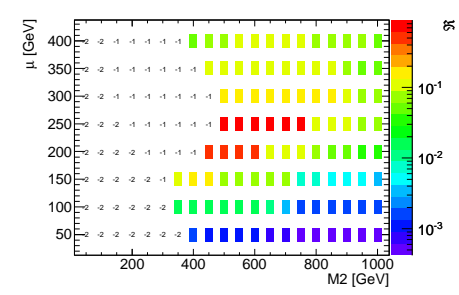

(d) $M_{1}=200 \mathrm{GeV}$

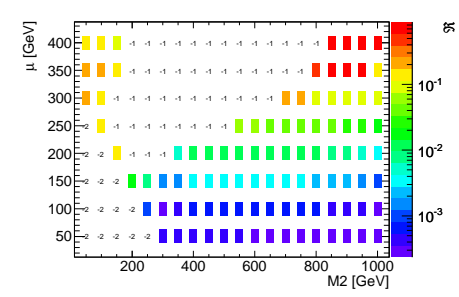

(g) $M_{1}=350 \mathrm{GeV}$

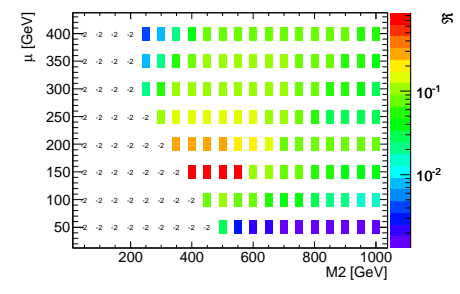

(b) $M_{1}=100 \mathrm{GeV}$

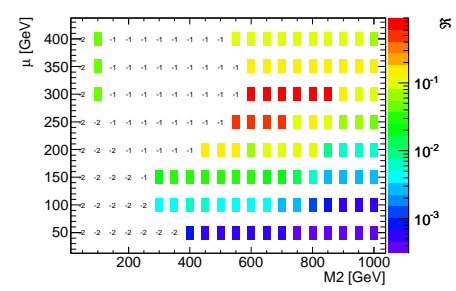

(e) $M_{1}=250 \mathrm{GeV}$

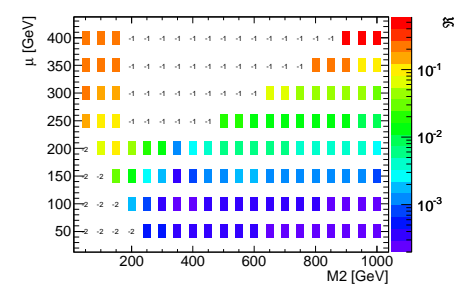

(h) $M_{1}=400 \mathrm{GeV}$

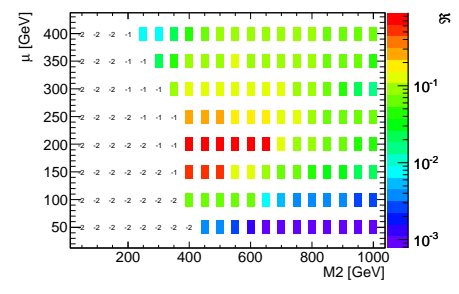

(c) $M_{1}=150 \mathrm{GeV}$

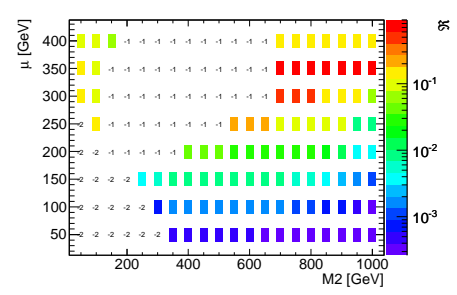

(f) $M_{1}=300 \mathrm{GeV}$

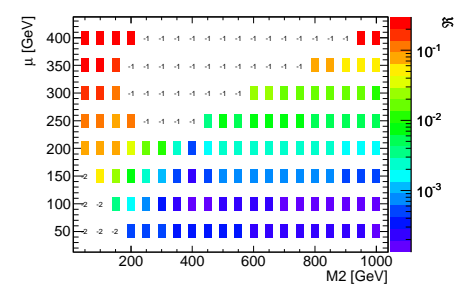

(i) $M_{1}=450 \mathrm{GeV}$

Figure 6.5: The ratio $\mathcal{R}$ as a function of $M_{2}$ and $\mu$ for different values of $M_{1}$. Small parts of the parameter space of the pMSSM show values of $\mathcal{R} \gtrsim 0.5$, such that a search with four leptons in the final state might be more sensitive to new physics than a search with three leptons in the final states in these regions.

A list of points that meet all criteria that were discussed above and that are therefore of interest for a search with four leptons in the final state is summarised in Table 6.1. The set of these points is referred to as $L$ in the following. As the final step in the construction of the simplified models for $\tilde{\chi}_{2}^{0}-\tilde{\chi}_{3}^{0}$ production, the characteristics of these points are now discussed in some detail.

In Figure 6.6 some of the most relevant characteristics have been summarised. The plot shown in Figure 6.6(a) indicates that for all points in $L$ the most relevant process is the associate production of a $\tilde{\chi}_{2}^{0}$ and a $\tilde{\chi}_{3}^{0}$. Also, all points in $L$ feature a $\tilde{\chi}_{4}^{0}$ that is almost exclusively Wino, as demonstrated in Figure 6.6(b); this means that the lighter mass eigenstates are mainly Binoand Higgsino- like. In Figure 6.6(c) the mass difference between the two produced neutralinos and the LSP is shown. Three distinct 'islands' emerge, corresponding to three distinct straight 


\begin{tabular}{|c|c|c|}
\hline M1 $[\mathrm{GeV}]$ & $\mathrm{M} 2[\mathrm{GeV}]$ & $\mu[\mathrm{GeV}]$ \\
\hline 100 & $400-550$ & 150 \\
150 & $400-500$ & 150 \\
150 & $400-650$ & 200 \\
200 & $400-450$ & 150 \\
200 & $450-600$ & 200 \\
200 & $500-750$ & 250 \\
250 & $450-550$ & 200 \\
250 & $550-700$ & 250 \\
250 & $600-850$ & 300 \\
300 & $550-650$ & 250 \\
300 & $700-800$ & 300 \\
300 & $700-1000$ & 350 \\
350 & $800-950$ & 350 \\
350 & $850-1000$ & 400 \\
\hline
\end{tabular}

Table 6.1: A list of all points in the studied part of the pMSSM for which a four lepton search might be more sensitive than a search with three leptons in the final state.

lines with a small width in Figure 6.6(d), which shows the correlation of the mass difference between the second lightest neutralino and the right chiral sleptons and the mass difference between the right chiral sleptons and the LSP. On the z-axis, the average Bino component of the LSP is shown. Note that in each bin the average has been calculated from a maximum of two points, while for the majority of the bins one bin corresponds to exactly one point. The three areas/lines correspond to the three different values of $M_{1}-\mu$ that are covered by the points in $L$, as emphasised in Figure 6.6(e). As shown in Figure 6.6(f), the LSP mass ranges from roughly $100 \mathrm{GeV}$ to about $350 \mathrm{GeV}$, whereas a continuation of the grid to larger values of $M_{1}, M_{2}$ and $\mu$ will enlarge the upper value. For $M_{1}-\mu=-50 \mathrm{GeV}$, the LSP is highly Bino like, and its mass is roughly given by $M_{1}$. If $\mu$ is decreased for a fixed $M_{1}$, the LSP gets a larger Higgsino component, while the $\tilde{\chi}_{3}^{0}$ gets more Bino like.

For all points in $L$, the decay chains

$$
\begin{array}{lll}
\tilde{\chi}_{3}^{0} & \rightarrow \tilde{l}_{R}^{ \pm} l^{\mp} \rightarrow \tilde{\chi}_{1}^{0} l^{ \pm} l^{\mp} & \text { and } \\
\tilde{\chi}_{2}^{0} & \rightarrow \tilde{l}_{R}^{ \pm} l^{\mp} \rightarrow \tilde{\chi}_{1}^{0} l^{ \pm} l^{\mp} &
\end{array}
$$

have a branching fraction of $100 \%$. Example mass spectra for some points in $L$ are shown in Figure 6.7. The first two examples show the mass spectra for two points with $M_{1}-\mu=50$ $\mathrm{GeV}$. The neutralino sector looks very similar for all of these points, i.e. the $\tilde{\chi}_{2}^{0}$ and the $\tilde{\chi}_{3}^{0}$ are nearly mass degenerate and much have a slightly larger mass than the light chargino. The mass difference between the heavier neutralinos and the sleptons depends on the value of $M_{2}$ : The larger $M_{2}$ gets, the larger get the slepton masses and the smaller gets the mass difference between the sleptons and the heavier neutralinos. The third example shows the mass spectrum for a point with $M_{1}=\mu$. For all those points in $L$ the mass difference between the $\tilde{\chi}_{2}^{0}$ and the $\tilde{\chi}_{3}^{0}$ gets slightly larger. Finally for $M_{1}-\mu=50 \mathrm{GeV}$, the mass difference between the LSP and the $\tilde{\chi}_{2}^{0}$ gets small, whereas the mass difference between the $\tilde{\chi}_{3}^{0}$ and the $\tilde{\chi}_{2}^{0}$ gets relatively large. 


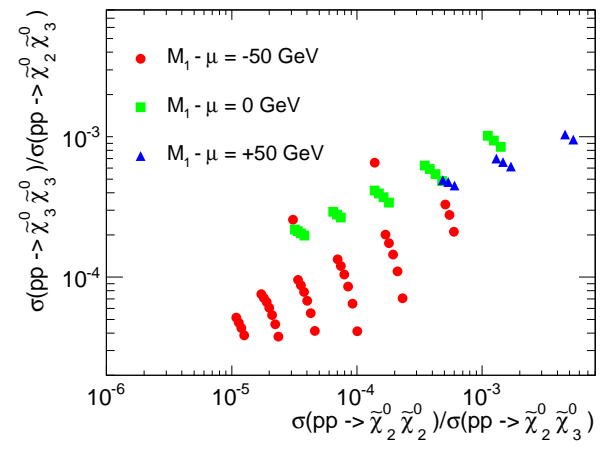

(a) Ratio of cross sections

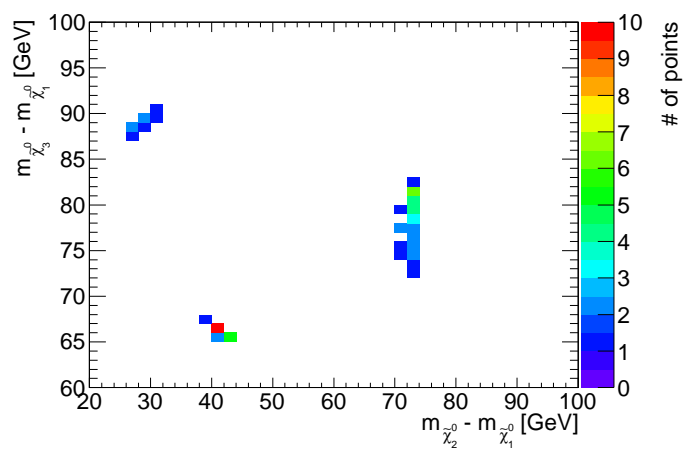

(c) Neutralino mass differences

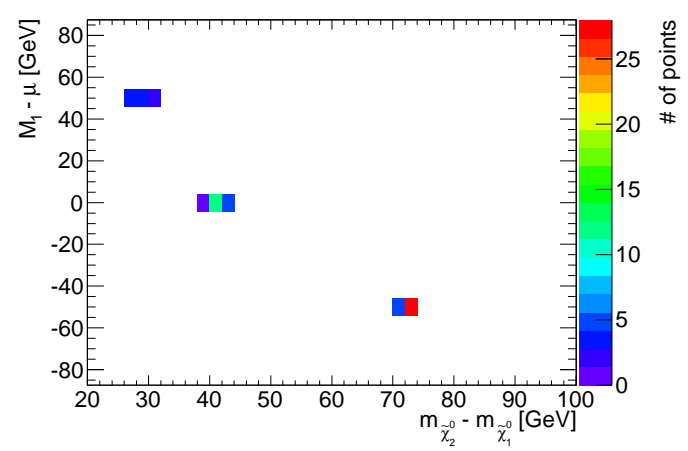

(e) Correlation between mass differences and parameters

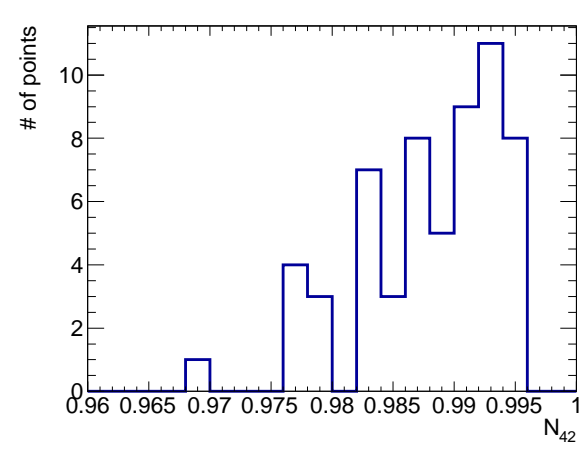

(b) Wino component of $\tilde{\chi}_{4}^{0}$

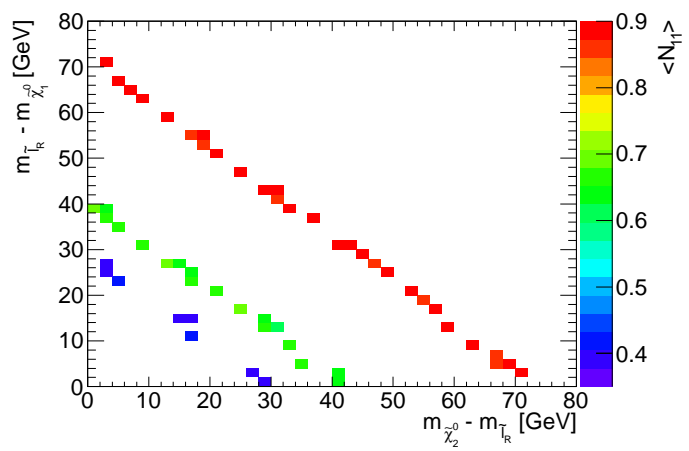

(d) Neutralino and slepton mass differences

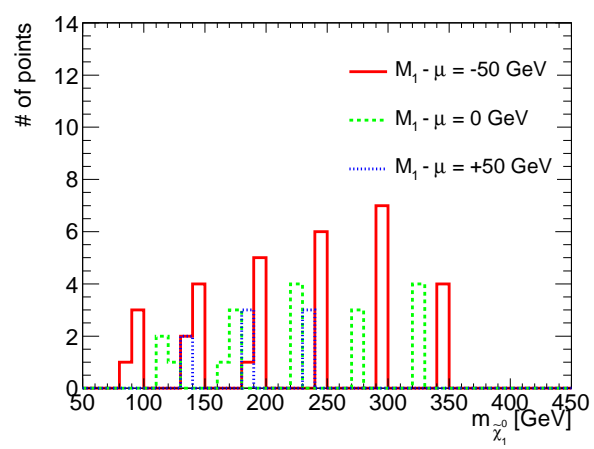

(f) LSP mass

Figure 6.6: Some characteristics of the points in the studied part of the pMSSM at which a search with four leptons might be more sensitive than a search with three leptons in the final state.

\subsubsection{The Simplified Model v2A}

A first simplified model for $\tilde{\chi}_{2}^{0}-\tilde{\chi}_{3}^{0}$ production has been designed very closely following the pMSSM with $M_{1}-\mu=-50 \mathrm{GeV}$. The reason for not considering the other potentially interesting points is the smaller mass differences between the second lightest neutralino and the right chiral sleptons 


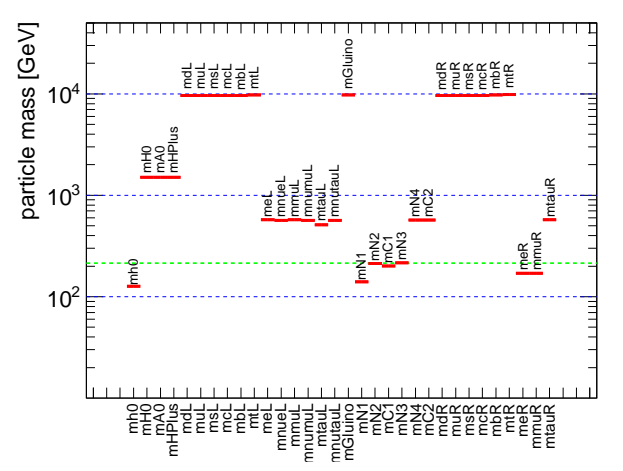

(a) $M_{1}=150 \mathrm{GeV}, M_{2}=500 \mathrm{GeV}, \mu=200 \mathrm{GeV}$

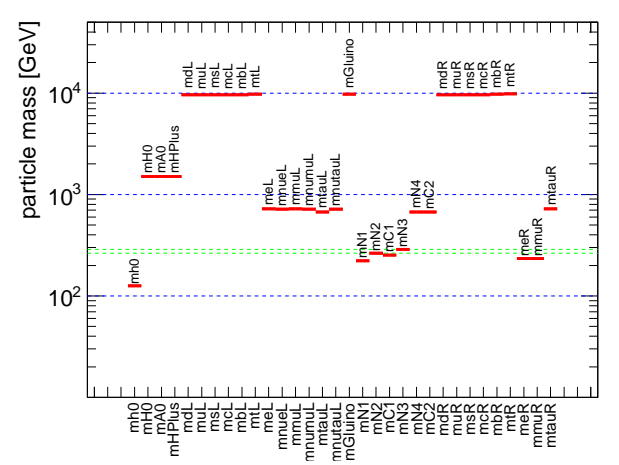

(c) $M_{1}=250 \mathrm{GeV}, M_{2}=600 \mathrm{GeV}, \mu=250 \mathrm{GeV}$

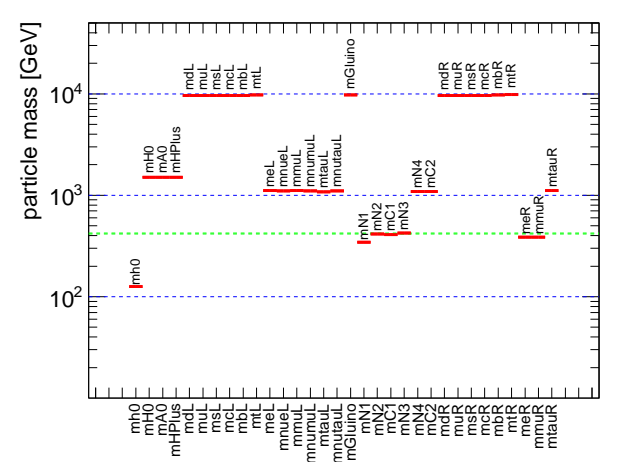

(b) $M_{1}=350 \mathrm{GeV}, M_{2}=1000 \mathrm{GeV}, \mu=400 \mathrm{GeV}$

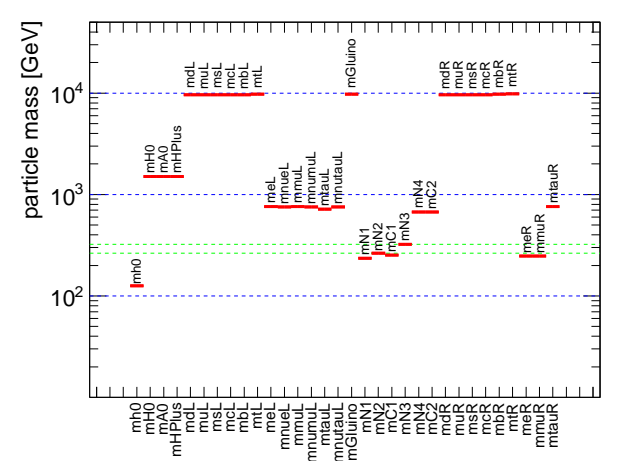

(d) $M_{1}=300 \mathrm{GeV}, M_{2}=600 \mathrm{GeV}, \mu=250 \mathrm{GeV}$

Figure 6.7: The mass spectra for four different points in $L$ as an example. Two examples for $M_{1}-\mu=-50 \mathrm{GeV}$ and one example for each $M_{1}=\mu$ and $M_{1}-\mu=50 \mathrm{GeV}$ have been considered.

at these points. An important factor that has not been taken into account for the considerations so far is the acceptance of the detector and the efficiency of the analysis cuts. In the searches for direct gauginos, signal leptons are required to have a transverse momentum of more than 10 $\mathrm{GeV}$ for several reasons (cf. section 6.3). With a mass difference of only $20-40 \mathrm{GeV}$ between the $\tilde{\chi}_{2}^{0}$ and the $\tilde{\chi}_{1}^{0}$, and the corresponding smaller mass differences between the neutralinos and the right chiral sleptons, the phase space for the leptons in the final state gets kinematically cut off at small transverse momenta, and the probability to reconstruct four leptons with a transverse momentum of more than $10 \mathrm{GeV}$ gets very small.

The masses of all superpartners except for the $\tilde{\chi}_{1}^{0}, \tilde{\chi}_{2}^{0}, \tilde{\chi}_{3}^{0}$ and the right chiral sleptons have been fixed to $100 \mathrm{TeV}$. Also, the mass of the lightest Higgs boson has been fixed to $125 \mathrm{GeV}$ and the masses of the heavier neutral and the charged Higgs bosons has been set to $1.5 \mathrm{TeV}$ (since the only process to be studied in the simplified model is the associate production of the Higgsino-like neutralinos via a Z boson and their decay via intermediate right chiral sleptons, the masses of the Higgs bosons and the other sparticles are in fact of no importance, here, and are only given for completeness).

The mass differences between the LSP and the heavier neutralinos has been fixed to

$$
m_{\tilde{\chi}_{2}^{0}}-m_{\tilde{\chi}_{1}^{0}}=75 \mathrm{GeV} \quad \text { and } \quad m_{\tilde{\chi}_{3}^{0}}-m_{\tilde{\chi}_{1}^{0}}=80 \mathrm{GeV}
$$


and the mass of the $\tilde{\chi}_{3}^{0}$ is used as a parameter,

$$
m_{\tilde{\chi}_{3}^{0}} \in\left[180 \mathrm{GeV}, m_{\tilde{\chi}_{3}^{0}}^{\max }\right]
$$

where the increment between two points is $50 \mathrm{GeV}$. With the exception of the two points with $M_{2}=400 \mathrm{GeV}$, for all points in $L$ with $M_{1}-\mu=-50 \mathrm{GeV}$ each entry in the mixing matrix is very similar, such that the mixing matrix in the simplified model has been fixed:

$$
\mathbf{N}=\left(\begin{array}{cccc}
0.87 & -0.07 & 0.39 & -0.30 \\
-0.06 & 0.06 & 0.70 & 0.71 \\
-0.49 & -0.18 & 0.60 & -0.61 \\
-0.02 & 0.98 & 0.1 & -0.18
\end{array}\right)
$$

Thus, the simplified model features a heavy Wino-like neutralino, a light Bino-like neutralino, and two intermediate Higgsino-like neutralinos. Due to the different signs of $N_{24}$ and $N_{34}$, while the signs for $N_{23}$ and $N_{33}$ are the same, the vertex factor for the production via a $Z$ boson gets large, such that couplings ensure a reasonable production cross section.

Motivated by the pMSSM, the mass difference between the $\tilde{\chi}_{3}^{0}$ and the right chiral sleptons has been used as another parameter, such that for each value of $m_{\tilde{\chi}_{3}^{0}}$ the slepton masses

$$
m_{\tilde{l}_{R}} \in\left[m_{\tilde{\chi}_{3}^{0}}-15 \mathrm{GeV}, m_{\tilde{\chi}_{3}^{0}}-75 \mathrm{GeV}\right]
$$

in steps of $10 \mathrm{GeV}$ are considered. As in the pMSSM, the decay chains (6.4) and (6.5) have been assigned a branching fraction of $100 \%$. Apart from small differences in the mixing matrix, each point in the simplified model corresponds to a very similar point in the pMSSM - the points with the larger neutralino masses correspond to values of $M_{1}, M_{2}$ and $\mu$ beyond the scope of the studied part of the pMSSM, however.

Naturally the production cross section for a $\tilde{\chi}_{2}^{0}-\tilde{\chi}_{3}^{0}$ pair does not depend on that second parameter of the simplified model, but it does heavily depend on $m_{\tilde{\chi}_{3}^{0}}$, as shown in Figure 6.8(a), where LSP masses up to $700 \mathrm{GeV}$ are considered. As indicated above, the acceptance however does significantly depend on the mass splittings between the neutralinos and the right chiral sleptons. In order to assess the discovery reach in the simplified model phase space with the LHC and assign a reasonable value to $m_{\tilde{\chi}_{3}^{0}}^{\max }$ before using a huge amount of CPU time for the full simulation, a total of 10,000 events have been generated using the MadGraph event generator, taking into account initial and final state radiation. The MC truth information from these events has been used to roughly estimate the acceptance of a search with four leptons in the final state, and identify the limiting factors in the event selection.

The selection criteria have been kept very simple: Electrons and muons that originate from the decay of a $\tilde{\chi}_{i}^{0}$ or a $\tilde{l}_{R}$ are required to have a transverse momentum of more than $10 \mathrm{GeV}$ and electrons (muons) have to satisfy $|\eta|<2.47(|\eta|<2.4$ ). Furthermore, events with a lepton pair with the same flavour, opposite charge (SFOS) and an invariant mass of $m_{\mathrm{SFOS}}=m_{Z} \pm 10$ $\mathrm{GeV}$ are vetoed. This cut is commonly used in the ATLAS multilepton analyses to reduce the background from $W Z$ and $Z Z$ events. By construction, all events in the simplified model feature the existence of a SFOS lepton pair; the choices of $m_{\tilde{\chi}_{3}^{0}}-m_{\tilde{l}_{R}}$ ensure that the invariant mass of an SFOS pair in the same decay chain is not larger than roughly $75 \mathrm{GeV}$, such that only combinatorial background in events where all four leptons have the same flavour can potentially be rejected by this cut. Finally, the missing transverse energy at truth level has been required to exceed $50 \mathrm{GeV}$. This simplified 'signal region' is highly correlated to one of the signal regions that have been used for the RPV searches with four leptons in the final state before, although 


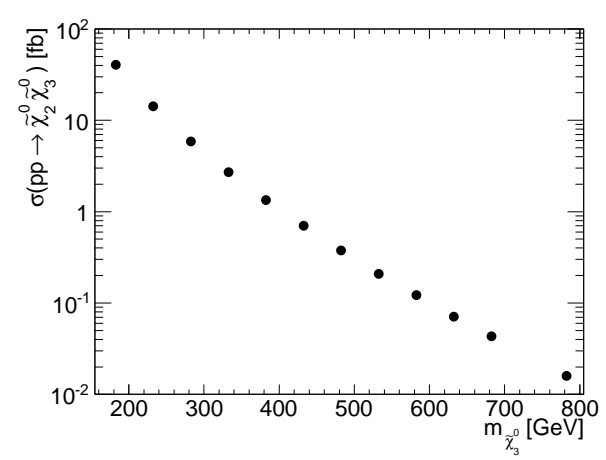

(a) Signal Cross Section v2A

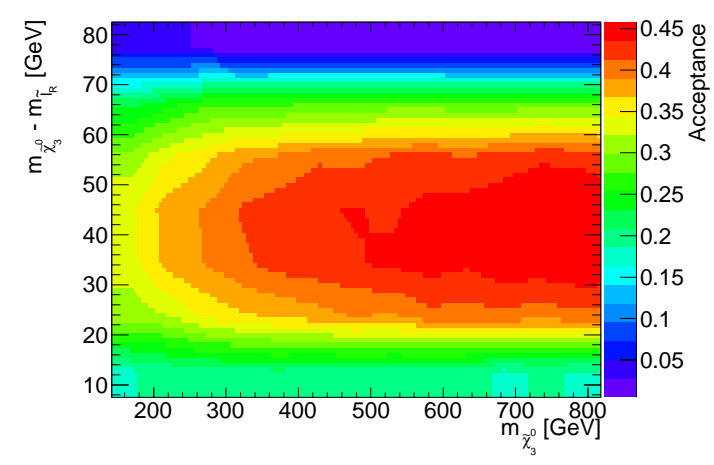

(c) Acceptance v2A

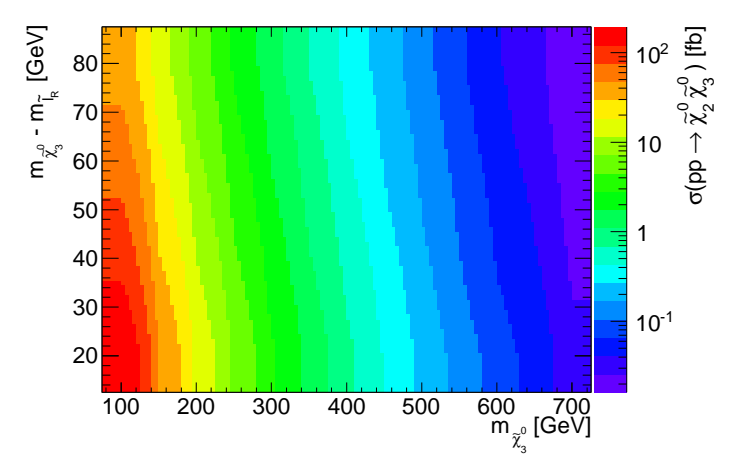

(b) Signal Cross Section v2B

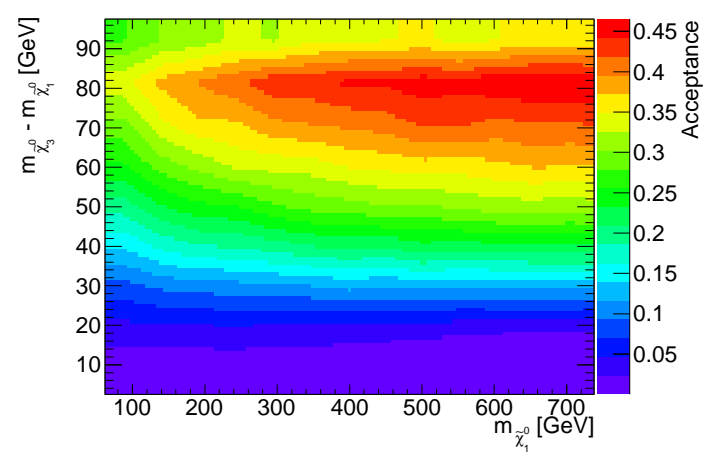

(d) Acceptance v2B

Figure 6.8: The production cross section for a $\tilde{\chi}_{2}^{0} \tilde{\chi}_{3}^{0}$ pair and the estimated acceptance based on generator level information for the simplified models $v 2 A$ and $v 2 B$. The production cross section depends highly on the mass scale in the neutralino sector, while the acceptance depends highly on the mass difference between the neutralinos and the sleptons, as well. For the simplified model $v 2 A$, the production cross section does not depend on the mass difference between the $\tilde{\chi}_{0}^{3}$ and the $\tilde{l}_{R}$.

the results from that search are not directly transferable [151]. The estimated acceptance using these cuts is shown in Figure 6.8(c).

For a constant mass difference between the $\tilde{\chi}_{3}^{0}$ and the $\tilde{l}_{R}$, one of the more important limitations on the acceptance arises from the $\eta$ distribution of the leptons, as exemplified in Figure 6.9(a) for a mass difference of $55 \mathrm{GeV}$. On the other axis, for a fixed mass of the $\tilde{\chi}_{3}^{0}$, it is the transverse momentum of the fourth lepton that limits the acceptance significantly, in particular for small mass differences between the neutralinos and the slepton, as shown in Figure 6.9(b). A significant part of the overall selection inefficiency arises due to the requirement on the $E_{T}$ to add up to more than $50 \mathrm{GeV}$. Naturally the $E_{T}$-spectrum tends to be harder for larger LSP masses, as shown in Figure 6.9(c). The cut efficiency depends also on the mass splitting between the neutralinos and the sleptons; if the slepton mass is close to the mass of the $\tilde{\chi}_{3}^{0}$ the slepton is not heavily boosted in the $\tilde{\chi}_{3}^{0}$-frame of reference; also, as in that case the mass difference between the slepton and the LSP is relatively large, the LSP can get a significant boost in the rest frame of the slepton, which will also result in a high LSP momentum in the detector frame. If the slepton mass is close to the LSP mass, the mass difference between the slepton and the 
$\tilde{\chi}_{3}^{0}$ is large, such that the slepton can be highly boosted in the detector frame; the LSP is only marginally boosted in the slepton rest frame, such that the probability to get a boosted LSP in the detector frame is high. For medium mass differences between the neutralinos and the sleptons, the momentum of the slepton in the rest frame of the $\tilde{\chi}_{3}^{0}$ is large, and also the momentum of the LSP in the slepton rest frame is large, and there is a considerable probability that these two effects counterbalance each other, resulting in a softer $\mathbb{E}_{T^{-}}$spectrum.

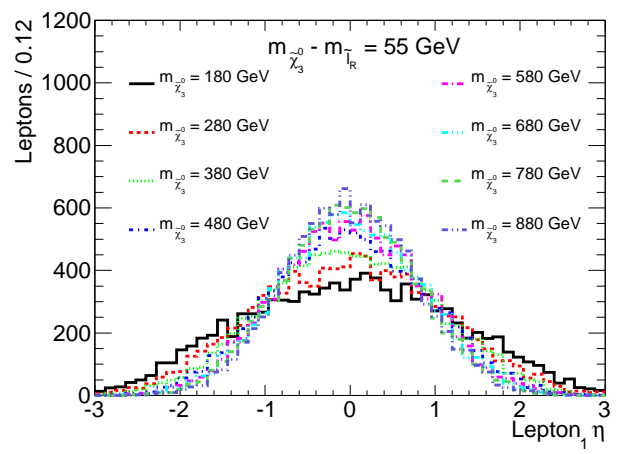

(a) leading lepton $\eta$

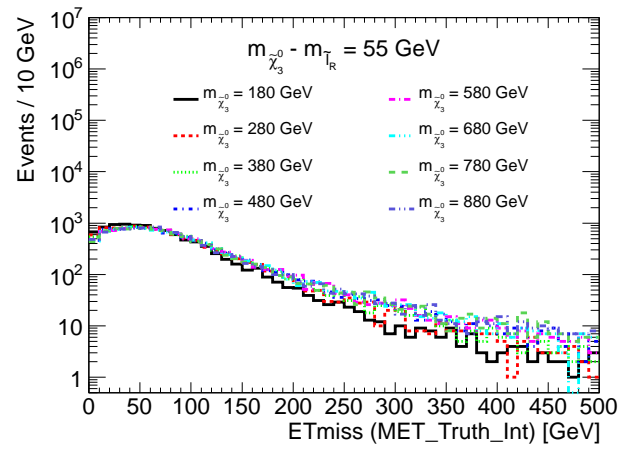

(c) $\mathbb{E}_{T}$ distribution as a function of $m_{\tilde{\chi}_{3}^{0}}$

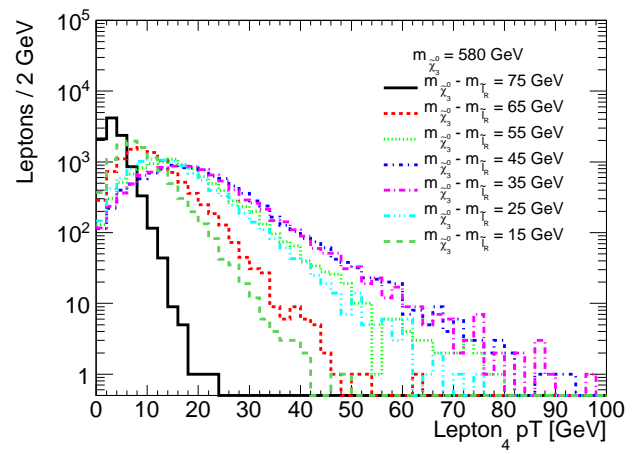

(b) $p_{T}$ of the lowest $p_{T}$ lepton

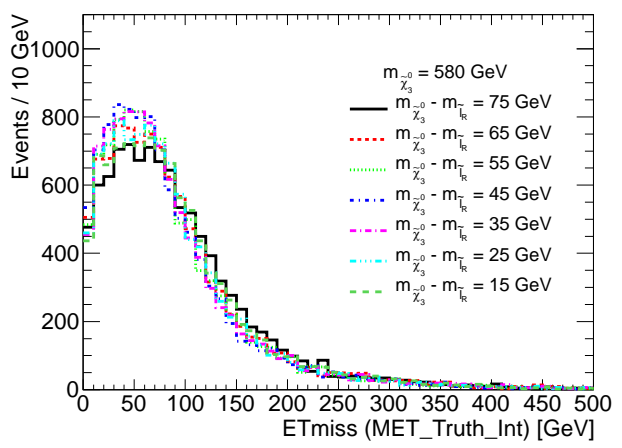

(d) $E_{T}$ distribution as a function of $m_{\tilde{\chi}_{3}^{0}}-m_{\tilde{l}_{R}}$

Figure 6.9: Several distributions limiting the acceptance in the simplified model v2A at different edges of the grid.

To conclude the discussion of the simplified model v2A and define the ultimate parameter boundaries, a simple estimation of the expected limit has been performed. Using the CLs technique, assuming a dataset of $30 \mathrm{fb}^{-1}$, a SM background of $1 \pm 1$ events in the signal region and a signal uncertainty of $30 \%$, the estimation for the expected limit is shown in Figure 6.10. The numbers are loosely based on the ATLAS search for RPV SUSY with four leptons in the final state [151] - the goal is not to provide a reliable estimate, but to define reasonable boundaries for the parameters of the simplified model grid before sending the grid into the CPU-consuming $\mathrm{MC}$ production.

Based on the estimated expected limit, $m_{\tilde{\chi}_{3}^{0}}^{\max }=780 \mathrm{GeV}$ has been chosen. 


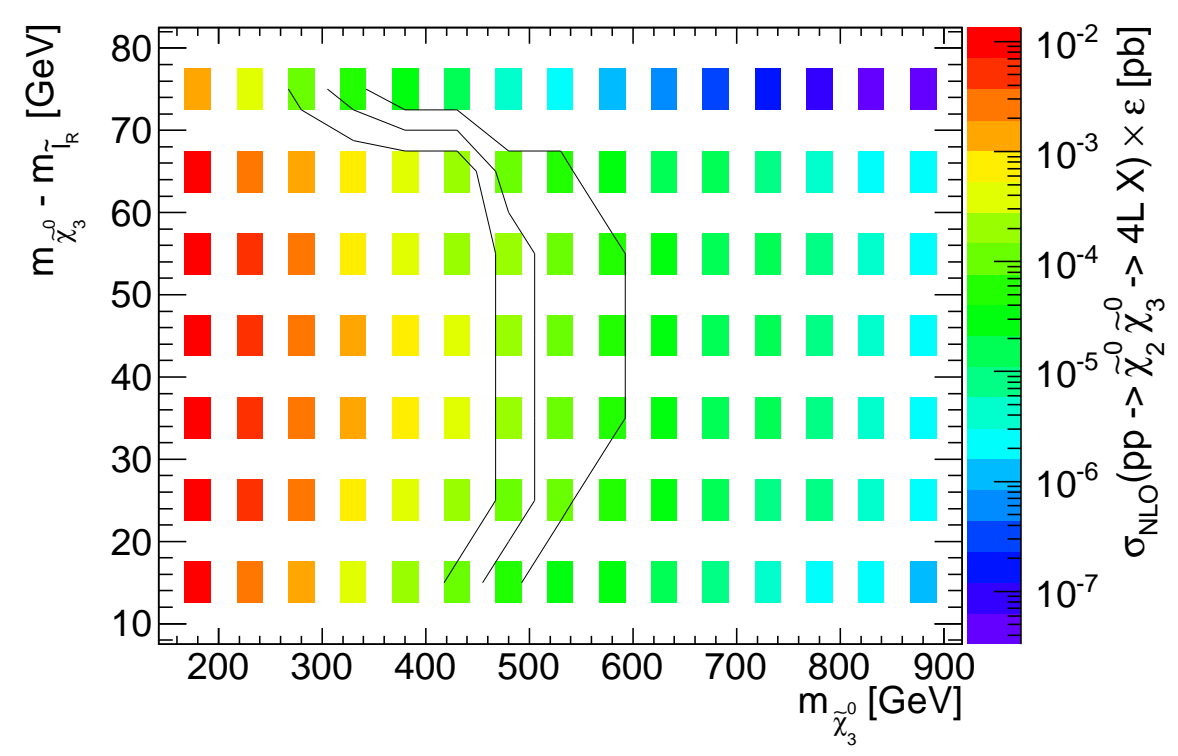

Figure 6.10: The total four lepton cross section times the estimated acceptance in the simplified model $v 2 A$. The black lines indicate the roughly estimated expected limit in this model, which has been used to define the parameter boundaries.

\subsubsection{The Simplified Model v2B}

As indicated by Figure 6.6(e) for instance, depending on the difference between $M_{1}$ and $\mu$, there are parts of the pMSSM in which a search with four leptons in the final state may be most sensitive compared to searches with a different lepton multiplicity, where the mass difference between the LSP and the heavier neutralinos is different from $\sim 80 \mathrm{GeV}$. Therefore a slightly differently parametrised second generation of a simplified model for $\tilde{\chi}_{2}^{0}-\tilde{\chi}_{3}^{0}$ production has been designed; this simplified model is not as closely related to the pMSSM as the above simplified model $v 2 A$.

The simplified model $v 2 B$ inherits most of its features from the simplified model $v 2 A$; the masses of all sparticles but the three lightest neutralinos and the right chiral sleptons have been fixed to $100 \mathrm{TeV}$, the branching fraction of the decays (6.4) and (6.5) has been set to $100 \%$, and the mixing matrix (6.8) has been used. The latter point is one of the features where the simplified model $v 2 B$ diverges from the pMSSM, where the mass differences between the neutralinos are heavily correlated with the mixing matrix.

The masses of the $\tilde{\chi}_{1}^{0}$ and the $\tilde{\chi}_{3}^{0}$ have been chosen as parameters of this simplified model, with

$$
\begin{aligned}
& m_{\tilde{\chi}_{1}^{0}} \in\left[100 \mathrm{GeV}, m_{\tilde{\chi}_{1}^{0}}^{\max }\right], \\
& m_{\tilde{\chi}_{3}^{0}} \in\left[m_{\tilde{\chi}_{1}^{0}}+\Delta m^{\mathrm{min}}, m_{\tilde{\chi}_{1}^{0}}+\Delta m^{\mathrm{max}}\right], \\
& m_{\tilde{\chi}_{2}^{0}}=m_{\tilde{\chi}_{3}^{0}} \quad \text { and } \\
& m_{\tilde{l}_{R}}=\frac{1}{2} \cdot\left(m_{\tilde{\chi}_{1}^{0}}+m_{\tilde{\chi}_{3}^{0}}\right) .
\end{aligned}
$$

The model is therefore designed to probe a diagonal with a small width in the $m_{\tilde{\chi}_{1}^{0}-} m_{\tilde{\chi}_{3}^{0}}$. The mass difference has been kept small because with the used mixing matrix, the decay of the 


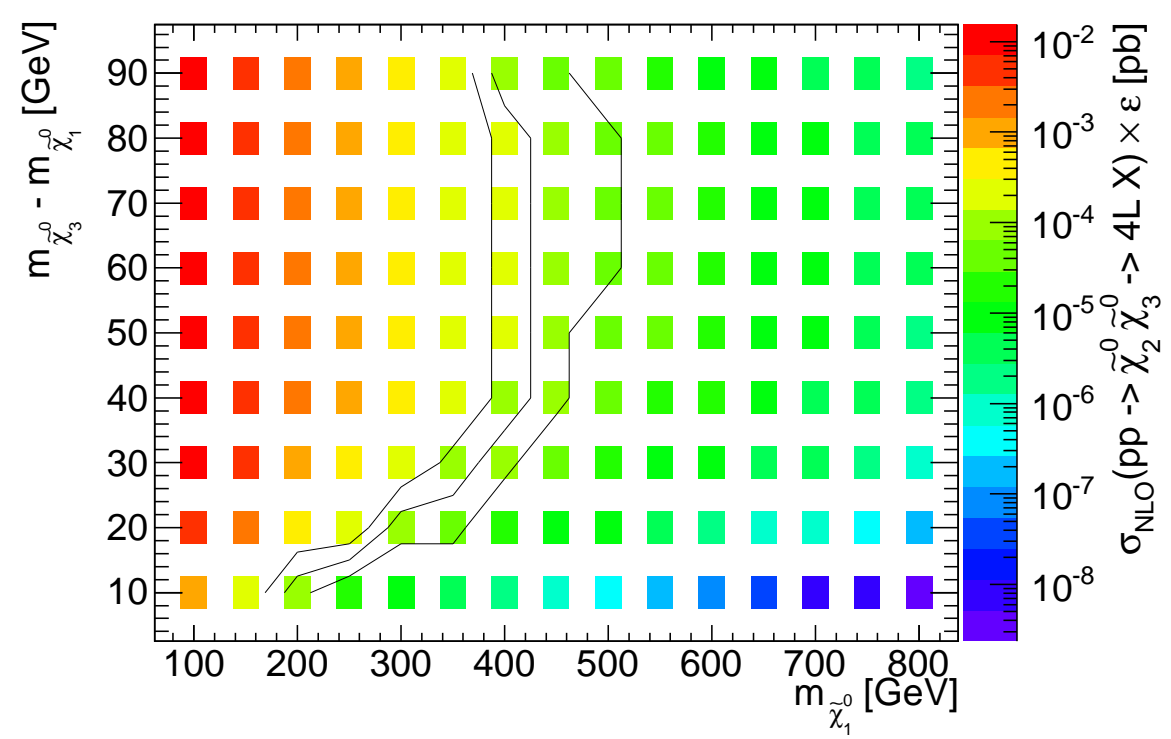

Figure 6.11: The total four lepton cross section times the estimated acceptance in the simplified model v2B. The black lines indicate the roughly estimated expected limit in this model, which has been used to define the parameter boundaries.

heavier neutralinos into a $Z$ boson and the LSP becomes dominant in any realistic model if it is allowed kinematically. While it tends to imply a softer $\mathbb{F}_{T}$-distribution, the mass difference between the right chiral sleptons and the neutralinos has been set to the maximum value for both of the subsequent decays that are relevant here - the reason is that a smaller or a larger mass of the slepton would lead to a softer $p_{T}$ spectrum for the leptons, which will tend to decrease the acceptance significantly. The production cross section for a $\tilde{\chi}_{2}^{0}$ in association with a $\tilde{\chi}_{3}^{0}$ in the simplified model v2B is shown in Figure 6.8(b); the estimated acceptance, using the same selection as described for the simplified model v2A, is shown in Figure 6.8(d).

Similar to the study that has been performed for the simplified model $v 2 A$, the $m_{\tilde{\chi}_{1}^{0}-m_{\tilde{\chi}_{3}^{0}}}$ plane has been scanned on the theory level in order to set reasonable values for the $m_{\tilde{\chi}_{1}^{0}}^{\max }$, $\Delta m^{\min }$ and $\Delta m^{\max }$. Using the same pseudo signal selection as in the simple study performed for the simplified model v2A, the results are summarised in Figure 6.11. Based on these results, $m_{\tilde{\chi}_{1}^{0}}^{\max }=700 \mathrm{GeV}, \Delta m^{\min }=20 \mathrm{GeV}$ and $\Delta m^{\max }=80 \mathrm{GeV}$ have been chosen for the full production of the model.

\subsection{Analysis Overview}

A dataset corresponding to a total integrated luminosity of $20.7 \pm 0.7 \mathrm{fb}^{-1}$ is analysed. The data was taken with the ATLAS detector during the LHC proton-proton run at a centre-of-mass energy of $\sqrt{s}=8 \mathrm{TeV}$ in 2012, using a combination of several single lepton and dilepton triggers for the online selection.

Four or more isolated light charged leptons with a transverse momentum of more than $10 \mathrm{GeV}$ are required in the offline selection. The cut on the transverse momentum limits the sensitivity of the analysis to scenarios with very small mass differences, but it enhances the suppression of SM background with fake leptons and ensures a good quality and understanding of reconstructed 
leptons. The decay chains in the signal models considered here are free of on-shell Z-bosons, such that events containing a leptonic $\mathrm{Z}$ candidate are vetoed (Z-veto).

While the analysis is designed to provide a high sensitivity to the production of $\tilde{\chi}_{2}^{0}-\tilde{\chi}_{3}^{0}$ pairs, the SM background can be highly suppressed with a limited number of cuts already. After requiring at least four leptons and the absence of any $\mathrm{Z}$ candidate in the event, the expectation value for the number of SM events in the analysed data sample becomes very small - at the order of 1 - already by demanding the missing transverse energy to be larger than $50 \mathrm{GeV}$. While the targeted models for $\tilde{\chi}_{2}^{0}-\tilde{\chi}_{3}^{0}$ production can easily motivate further constraints on candidate signal events, such as the abundance of two lepton pairs with the same flavour and opposite charge (SFOS), these constraints are not imposed due to the small number of expected $\mathrm{SM}$ events in the $E_{T}>50 \mathrm{GeV}$ region. This allows for an easy re-interpretation of the search results in new physics scenarios with slightly different signatures - for instance, a different lepton composition in the final state - at a later point. It is for the same reason that the number of leptons required in the final state is inclusive.

The SM background is generally divided into two different types of events; those with four prompt leptons (irreducible background) and those with one or more fake leptons (reducible background).

The largest contribution to the irreducible SM background consists of events in which a top quark pair is produced in association with one or more massive gauge bosons, mainly $t \bar{t} Z$ and $t \bar{t} W W$ production. Another significant contribution to the irreducible SM background arises from the associate production of two or more massive electroweak gauge bosons. This is dominantly the production of two $Z$ bosons. Also, events in which a Higgs boson is produced and decays into two $Z$ bosons contributes to the background. The production of three massive gauge bosons does as well contribute to the SM background with four prompt leptons, mainly the associate production of a $Z$ boson with two $W$ bosons.

The dominant sources for reducible background are processes with three prompt leptons and one additional fake lepton in the final state, like the production of a $W$ boson in association with a $Z$ boson and jets. The contributions from other processes become smaller the smaller the multiplicity of prompt leptons get.

\subsection{Object Reconstruction and Event Preselection}

The ATLAS software release 17 has been used for the analysis presented here. Objects are selected according the the common recommendations of the ATLAS SUSY working group [152]; events are preselected according to these, as well. The most important selection criteria are described in this section.

\subsubsection{Data Sample}

For the results presented in this document, a total of $20.7 \mathrm{fb}^{-1}$ of proton-proton collision data were taken into account. This corresponds to the amount of data that was recorded with the ATLAS detector under nominal conditions during periods A-L of the 2012 LHC proton-proton run. Data that was acquired during intervals in which subsystems of the ATLAS detector were not operating under nominal conditions is rejected using a Good Runs List (GRL), which is provided centrally by the ATLAS SUSY Working Group. 


\subsubsection{Trigger}

Events are selected from the physics_Egamma and the physics_Muons stream. The appropriate amount of signal leptons, as defined below, are required to match an online object that is associated to one of the used triggers within $\Delta R=0.15$, where a constraint on the transverse momentum of the offline objects is imposed in order to ensure that only objects in the plateau region of the triggers are assigned a match. A logical OR of all single lepton and dilepton triggers that are listed in Table 6.2 is used - if at least one of the triggers was passed, the event is selected.

The technique described in chapter 5 is not applied - the reason is that the average trigger efficiency for events with four leptons with the used trigger composition in this analysis is very close to 1 in both data and also for most of the relevant MC samples - therefore, the MC is not re-weighted, and for technical reasons, the trigger simulation is used.

\begin{tabular}{c|c|c} 
Trigger Item & Targeted Object (s) & offline threshold [GeV] \\
\hline EF_e24vhi_medium1 & single, isolated electron & 25 \\
EF_mu24i_tight & single, isolated muon & 25 \\
EF_2e12Tvh_loose1 & two electrons & 14,14 \\
EF_e24vh_medium1_e7_medium1 & two electron & 25,10 \\
EF_2mu13 & two muons & 14,14 \\
EF_mu18_tight_mu8_EFFS & two muons & 18,10 \\
EF_e12Tvh_medium1_mu8 & one electron, one muon & 14,10 \\
EF_mu18_tight_e7_medium1 & one electron, one muon & 18,10
\end{tabular}

Table 6.2: The triggers used in this analysis. For the selection of signal events, a logical OR of all listed triggers is used.

\subsubsection{Cleaning Cuts}

Events are subject to a series of quality cuts to ensure a reasonable data integrity in the selected events.

Defects in the hardware, as well as non-collision backgrounds, such as showers from cosmicrays, can mimic a jet signature in the detector. A selection of jet moments is used to identify such 'bad' jets, and events with one or more 'bad' jets are vetoed, if that jet has a transvserse momentum of more than $20 \mathrm{GeV}$ and if it is not removed due to an overlapping electron. This quality cut is applied in both data and MC, and the looser definition [153] for a bad jet is used.

In a small fraction of the data taken during period $\mathrm{B}$, a hot spot in the tile calorimeter was not properly masked. An event is therefore rejected, if it contains a jet pointing to that hot spot. This cut is not applied in the MC, as only a small fraction of the data is subject to this cut and the impact on the integrated luminosity is not significant.

Events are also removed from the selection if they contain fake $\mathbb{E}_{T}$ due to malfunctioning cells in the tile calorimeter and the HEC. Such events are identified via jets with $p_{T}>40 \mathrm{GeV}$, if they are aligned to the $\mathbb{E}_{T}$. Events with noise bursts and corrupted data integrity in the LAr calorimeter or the tile calorimeter are rejected as well.

Due to the procedure used to reset the Timing, Trigger and Control signals, for some events a fraction of the full detector information may be missing. Such events are flagged and vetoed. Since the number of these events is very small, the total integrated luminosity is not corrected for this cut. 
Finally, a selection of quality cuts is applied to suppress non-collision background. First, the primary vertex in the event must have five or more associated tracks. Second, if there is a muon in the event with either a longitudinal impact parameter $\left|z_{0}\right|>1 \mathrm{~mm}$ or a transverse impact parameter $\left|d_{0}\right|>0.2 \mathrm{~mm}$ after overlap removal, the event is vetoed to suppress cosmic background. Third, in order to avoid the abundance of mis-measured muons in the event, for all muons the relation $\frac{\sigma_{q / p}}{|q / p|}<0.2$ must hold.

\subsubsection{Object Reconstruction}

The selection of physics objects - electrons, muons, taus, and jets - is performed in several steps. First, baseline objects are selected, by applying a series of basic cuts for each type of object to the reconstructed raw object candidates. In a second step, overlapping objects are removed, as are leptons from low mass resonances. In a final step baseline objects that pass the overlap and low mass removal are subject to further cuts by which signal objects are defined.

\section{Electrons}

Electron candidates are reconstructed with the egamma algorithms based on clusters in the ECAL and tracks from the ID [144]. Baseline electrons are selected from the electron candidates by requiring that

- the transverse energy is larger than $10 \mathrm{GeV}$,

- the absolute value of the pseudorapidity of the cluster is $\left|\eta^{c l}\right|<2.47$,

- the electron candidate matches the medium++ quality criterion [154], which is based on the shower shape in the ECAL, track-cluster matching, track properties and leakage into the hadronic calorimeters,

- the electron candidate has been reconstructed by an algorithm optimised for high $p_{T}$ electrons,

- the electron candidate has not deposited energy in dead or malfunctioning calorimter regions.

Signal electrons are selected from baseline electrons that are not removed due to an overlap with other objects, as explained further below, by requiring that

- the baseline electron passes the tight++ quality criteria,

- the significance of the transverse impact parameter is $\frac{\left|d_{0}\right|}{\sigma_{d_{o}}}<5$,

- $\left|z_{0} \sin \theta\right|<0.4 \mathrm{~mm}$,

- the electron is isolated. For electrons, isolation is defined by two quantities. First, the sum of the transverse momentum of all tracks in a cone of $\Delta R<0.3$ around the electron track must be $p_{T}^{\text {cone } 30}<0.16 \cdot E_{T}$, where $E_{T}$ is the transverse energy of the electron. In order to increase sensitivity to boosted scenarios, tracks of baseline leptons are not included in the $p_{T}^{\text {cone } 30}$ variable used in this analysis. Second, the energy deposited in a cone of $\Delta R$ around the electron is required to be $E_{T, c o r r}^{c o n e}<0.18 \cdot E_{T}$, where the index corr indicates that a vertex correction is applied to the energy deposited in the cone. This correction is $20.15 \mathrm{MeV}$ (17.94 MeV) per vertex with five or more associated tracks in data (MC), and it is subtracted from the raw energy deposit around the electron. 


\section{Muons}

Muon candidates are reconstructed from tracks in the ID and tracks or track segments in the MS using a statistical combination of the tracks (STACO algorithm). Combined muons are reconstructed from tracks in the ID and the MS, whereas segment tagged muons are reconstructed from tracks in the ID and track segments in the MS [144]. In MC, the transverse momentum of both types of muons is smeared to match the resolution observed in data [155, 156]. Muon candidates are subject to various cuts to define baseline muons:

- The transverse momentum is required to be larger than $10 \mathrm{GeV}$.

- The absolute value of the pseudorapidity must be smaller than 2.4.

- The ID track must pass a series of quality cuts: There must be at least one hit in the b-layer, if expected, and the track must have been reconstructed using at least one hit in the pixel detector and six hits in the SCT (if the tracks cross a dead pixel sensor or a dead SCT sensor, these count as hits). The number of holes in the pixel detector and the SCT must be less than three. Finally, for $0.1<|\eta|<1.9$, the sum of hits and outliers in the TRT must be larger than five. In any case, if that sum is larger than five, the number of outliers in the TRT must be smaller than nine times the number of hits in the TRT.

Signal muons are selected from baseline muons that are not discarded in the overlap removal. In addition to the baseline cuts, the following cuts are applied to define signal muons:

- The $d_{0}$ significance must be less than 3 .

- $\left|z_{0} \sin \theta\right|<1 \mathrm{~mm}$.

- Signal muons are required to be isolated. The sum of the transverse momentum of all tracks in a cone of $\Delta R<0.3$ around the muon has to be $p_{T, c o r r}^{\text {cone }}<0.12 \cdot p_{T}$, where $p_{T}$ is the transverse momentum of the muon. Similar to the procedure for the electron $E_{T, c o r r}^{\text {cone } 30}$, a vertex correction is applied to the sum of all track momenta. A total of 10.98 $\mathrm{MeV}(6.27 \mathrm{MeV})$ per vertex with more than five associated tracks is subtracted, as are the contributions from other leptons in order to ensure a good sensitivity to boosted scenarios.

\section{Jets}

The anti- $k_{T}$ algorithm [146] with a distance parameter of $\Delta R=0.4$ is used to reconstruct jets from topological clusters, employing local cluster calibration (LC). The energy measured from the calorimeters is corrected by applying the jet energy scale (JES), such that the jets used in this analysis are calibrated according to the LC + JES. Baseline jets are defined from all jet candidates by requiring

- the jet $p_{T}$ to be larger than $20 \mathrm{GeV}$ and

- the jet to be within $|\eta|<4.5$.

To define signal jets, remaining jets after the overlap removal are required to

- lie within $|\eta|<2.5$ and

- have a jet vertex fraction of JVF> 0.5. The JVF is the fraction of all track momenta associated to the jet that can as well be associated to the primary vertex.

For completeness, a signal jet is tagged as a b-jet using the MV1 algorithm [157]. An operating point with an average b-tagging efficiency of $85 \%$ is used. However, for the part of the search that is presented in this thesis, b-tagging is irrelevant. 


\section{Taus}

Taus are reconstructed from jet candidates with $p_{T}>10 \mathrm{GeV}$ and $|\eta|<2.5$, but the energy is corrected using the tau energy scale (TES) [158]. While in this analysis the signal region is completely blind to signal taus, tau candidates still have to be taken into account in the overlap removal, as the presented analysis is only a part of a more comprehensive analysis with signal regions that are sensitive to taus. Baseline taus are further required to

- have $p_{T}>20 \mathrm{GeV}$,

- have exactly 1 or 3 associated tracks,

- have a charge of exactly \pm 1 and

- fullfil loose tau identification criteria (using a boosted decision tree).

Baseline taus that are not rejected due overlapping other objects are identified as signal taus if

- they fulfill medium tau identification criteria.

However, signal taus are of no relevance for this analysis.

\section{Missing Transverse Energy}

The missing transverse energy is calculated using calibrated electrons, photons, muons, jets and topological clusters that are not associated to any of these objects. Electrons passing the medium++ quality cuts with a transverse energy of more than $10 \mathrm{GeV}$ are considered, as are both isolated and non-isolated muons (using the STACO muon collection). Photons must pass the tight selection criteria and have a transverse energy of more than $10 \mathrm{GeV}$. Jets with a transverse momentum of more than $20 \mathrm{GeV}$ are taken into account, where local cluster calibration is applied. Additional topological clusters and low energy deposits in the calorimeters are added to a so-called soft term $[159,160]$. The missing transverse energy is then given by

$$
\begin{aligned}
E_{x, y}^{\mathrm{miss}} & =E_{x, y}^{\mathrm{el}}+E_{x, y}^{\mu}+E_{x, y}^{\gamma}+E_{x, y}^{\mathrm{jet}}+E_{x, y}^{\mathrm{soft}}, \\
E_{T} & =\sqrt{E_{x}^{\mathrm{miss}^{2}}+E_{y}^{\mathrm{miss}^{2}}} .
\end{aligned}
$$

\section{Overlap Removal and Low Mass Resonances}

It may happen for several reasons that reconstructed baseline objects overlap, i.e. they are close to each other in terms of the distance measure $\Delta R$. Depending on the distance and the type of the overlapping objects, some of these are removed from the further analysis. This is done using the baseline objects as defined above in the following order, where objects that are removed in one step are no longer considered in the following steps:

- In case two baseline electrons are reconstructed within a distance of $\Delta R<0.1$, the electron with the lower energy is removed.

- Jets that lie within $\Delta R<0.2$ of a remaining baseline electron are removed. This is done because electrons are identified by both the electron and jet reconstruction algorithms.

- Taus are removed if they are within $\Delta R<0.2$ of a remaining electron or a baseline muon. 
- Since taus are as well identified as jets, remaining jets are removed if they are close to a remainig tau $(\Delta R<0.2)$.

- Electrons and muons that are within $\Delta R<0.4$ of a remaining jet are removed. This can be interpreted as an additional isolation requirement on the leptons, as this requirement mainly affects light leptons from heavy flavour jets - most of these would also be rejected in the signal lepton selection, but a fraction might pass the isolation requirements for signal leptons, such that this step in the overlap removal increases the purity of the signal leptons.

- In order to avoid using badly measured muons and electrons, both a remaining electron and a remaining muon are vetoed if they overlap within $\Delta R<0.1$. Such electrons are likely to be reconstructed due to photons originating from muon bremsstrahlung in the detector, where the cluster in the ECAL is matched to the muon track.

- Finally, muon pairs that are within a distance of $\Delta R<0.05$ are also removed.

In order to suppress leptons from low mass hadronic resonances and Drell-Yan processes, remaining baseline SFOS lepton pairs are removed from the selection if they have an invariant mass of less than $12 \mathrm{GeV}$.

\subsection{MC samples}

Several MC samples have been used for the optimisation of the signal region and for the estimation of the irreducible SM background. The ATLAS Underlying Event Tune 2B [161] has been used for the production of all used MC samples. Most samples that have been generated with the ALPGEN [162], POWHEG [163, 164, 165] and MadGraph [166] event generators use PYTHIA [167] for the parton shower. Samples generated with MC@NLO [168] as well as a few of the samples generated with ALPGEN use HERWIG [169] for the hadronisation and JIMMY [170] to model the underlying event. For the production of the ALPGEN and MadGraph samples, the PDF set CTEQ6L1 [171] has been used, whereas all other samples have been generated using the CT10 [172] PDF set.

\subsubsection{SM MC}

The nominal samples for the SM diboson processes were generated using the SHERPA [173] event generator. For the process $p p \rightarrow l l l l+X$, where $X$ is a collection of up to three jets, both t-channel $Z Z$ production as well as internal conversion in the final state are taken into account. All four leptons in the final state are required to have $|\eta|<2.7$ and $p_{T}>7 \mathrm{GeV}$; events with SFOS lepton pairs with an invariant mass of down to $8 \mathrm{GeV}$ have been generated. For the process $p p \rightarrow l l l \nu$ and $p p \rightarrow l l \nu \nu$, two of the leptons in the final state are required to have $|\eta|<2.7$ and $p_{T}>5 \mathrm{GeV}$. The minimal invariant mass of an SFOS pair was set to $0.2 \mathrm{GeV}$. All diboson samples are normalised to NLO by taking the ratio of the LO Sherpa cross section with the NLO MCFM $[174,175]$ cross section, where the filter cuts have been slightly adapted to MCFM.

For processes including three gauge bosons, such as $p p \rightarrow W W W, p p \rightarrow Z W W$ and $p p \rightarrow$ $Z Z Z$, the MadGraph generator has been used. For this analysis, the samples have been normalised to the NLO cross sections with the appropriate k-factors [176].

MadGraph has as well been used for the generation of events with a top quark pair and two additional $W$ bosons. For samples modelling the production of a top quark pair in association 
with one $W$ boson or one $Z$ boson, ALPGEN has been used. In these samples, the invariant mass of the SFOS pair originating from the $Z$ boson was required to be larger than $5 \mathrm{GeV}$ at generator level. Also for these processes, the samples are normalised to NLO using appropriate k-factors $[177,178]$.

Events in which a SM Higgs boson is produced via two gluons or by vector boson fusion are generated with POWHEG. The decay of the Higgs boson into two Z bosons is enforced in these samples. In addition, PYTHIA has been used to generate events for the associate production of a Higgs boson with a vector boson; finally, also the associate production of top quark pair with a Higgs boson is considered.

A more detailed overview on the used MC samples can be found in [147].

\subsubsection{Signal MC}

The MC samples for the two $\tilde{\chi}_{2}^{0} \tilde{\chi}_{3}^{0}$ signal grids were produced using MadGraph and PYTHIA. These samples are normalised to NLO using cross sections calculated with prospino. Different sets of PDFs and a variation of the factorisation and renormalisation scale are taken into account to estimate a theory uncertainty on the signal cross section.

\subsubsection{Corrections to the MC}

The MC simulation is tuned to data using event weights. Apart from the proper luminosity scale factor, there are two more factors that are relevant for this analysis. First, the distribution of the average number of interactions per bunch crossing in data is different from that in the MC. Therefore a pile-up reweighting is applied, which attributes a weighting factor to each event in the MC, such that the weighted distribution of the average number of interactions per bunch crossing in the MC agrees with the actual distribution in data.

Second, the lepton reconstruction efficiency is different in data and MC. Scale factors are measured by the ATLAS combined performance groups, and are applied to selected signal leptons in the MC.

\subsection{Signal Region}

The definition of the signal region for the $\tilde{\chi}_{2}^{0} \tilde{\chi}_{3}^{0}$ grids is performed using MC for the background estimation, although the reducible background is eventually estimated from data. Events are required to pass all cleaning cuts as outlined above. In addition, events must contain at least four signal leptons, where events with additional 'loose' light leptons are rejected. A 'loose' lepton is a baseline lepton that passes the overlap removal, but fails at least one of the subsequent signal lepton cuts. This step is necessary for the data driven background, estimation which is outlined below to yield appropriate results.

Further, events are required not to contain a leptonic $\mathrm{Z}$ candidate. This is defined as an SFOS lepton pair with an invariant mass of $\left|m_{S F O S}-m_{Z}\right| \leq 10 \mathrm{GeV}$. In addition, events are rejected if they contain an SFOS pair, which in combination with any remaining lepton in the event has an invariant mass of $\left|m_{S F O S+l}-m_{Z}\right| \leq 10 \mathrm{GeV}$. Also, events with two SFOS pairs are rejected, if $\left|m_{S F O S+S F O S}-m_{Z}\right| \leq 10 \mathrm{GeV}$. These cuts suppress radiative $\mathrm{Z}$ decays, like $Z \rightarrow l l \gamma$, and in particular $Z \rightarrow l l l l$, where in some cases one of the additional leptons gets a significant fraction of the $\mathrm{Z}$ momentum and the second additional lepton is not reconstructed for this reason.

This cut is motivated by the absence of $Z$ bosons in signal events. Also, the mass hierarchy of the neutralinos and the sleptons in the signal models that are considered here put a natural 
upper bound on the invariant mass of an SFOS pair in a signal event, which is given by the mass difference between the $\tilde{\chi}_{3}^{0}$ and the $\tilde{\chi}_{1}^{0}$. Depending on the mass of the intermediate sleptons, the invariant mass of the two leptons originating from the same $\tilde{\chi}_{3 / 2}^{0}$ must be smaller than at most $80 \mathrm{GeV}$, as this is the maximum splitting between the neutralino masses that are relevant here. While wrongly assigned SFOS pairs in signal events where all four leptons have the same flavour, can still populate the vetoed region, the fraction of such signal events is small. Also, the width of both the heavy neutralinos and the sleptons, as well as detector resolution effects may wash out the sharp threshold at $\lesssim 80 \mathrm{GeV}$; nevertheless, the probabilty that a $\mathrm{Z}$ candidate is reconstructed in a signal event is relatively small.

In contrast to some of the SM processes with four or more leptons in the final state, the signal events are expected to have a certain amount of real missing transverse energy due to the two neutralinos in the final state. While there is a non-negligible chance that the neutralinos leave the detector back-to-back, which would result in a very small amount of real missing transverse energy, the overall $\mathbb{E}_{T}$-spectrum is expected to be harder in signal events than in background events. The distribution of the reconstructed missing transverse energy for events with four or more signal leptons and no additional 'light' leptons is shown in Figure 6.12(a). Depending on the masses of the neutralinos, the expected number of signal events becomes dominant for $E_{T} \gtrsim 40 \mathrm{GeV}$.

In order to identify the optimal cut on the missing transverse energy, the expected significance is considered as a function of the $\mathbb{E}_{T}$ threshold. The significance $Z_{N}$ is defined as

$$
Z_{N 50}=\sqrt{2} \cdot \operatorname{erf}^{-1}\left(1-2 p_{0}\right)
$$

where $p_{0}$ is the p-value for the 'background only' hypothesis, assuming that the number of events measured in data corresponds to the expected number of background events plus the expected number of signal events for a given point. The index 50 indicates that a conservative $50 \%$ uncertainty on the background expectation was included. The significance is calculated at each point in the two considered signal grids, using a total of 100, 000 pseudo experiments to ensure a reasonable precision. The expected significance for four reference points is shown in Figure 6.12(b) as a function of the cut on the missing transverse energy. The four reference points are:

- v2A RP1: $m_{\tilde{\chi}_{3}^{0}}=280 \mathrm{GeV}, m_{\tilde{l}_{R}}=245 \mathrm{GeV}, m_{\tilde{\chi}_{1}^{0}}=200 \mathrm{GeV}$

- v2A RP2: $m_{\tilde{\chi}_{3}^{0}}=330 \mathrm{GeV}, m_{\tilde{l}_{R}}=285 \mathrm{GeV}, m_{\tilde{\chi}_{1}^{0}}=250 \mathrm{GeV}$

- v2B RP1: $m_{\tilde{\chi}_{3}^{0}}=265 \mathrm{GeV}, m_{\tilde{l}_{R}}=232.5 \mathrm{GeV}, m_{\tilde{\chi}_{1}^{0}}=200 \mathrm{GeV}$

- v2B RP2: $m_{\tilde{\chi}_{3}^{0}}=185 \mathrm{GeV}, m_{\tilde{l}_{R}}=167.5 \mathrm{GeV}, m_{\tilde{\chi}_{1}^{0}}=150 \mathrm{GeV}$

Naturally a cut that is optimal for one reference point does not necessarily coincide with the optimal threshold for a different reference point. In general, it has been found that a cut at 50 $\mathrm{GeV}$ or $60 \mathrm{GeV}$ seems optimal. Given that for points with a higher expected significance at 60 $\mathrm{GeV}$ the loss in the expected significance is marginal if the cut is moved to $50 \mathrm{GeV}$, and given that for some points the decrease in the expected significance is considerable when moving from $50 \mathrm{GeV}$ to $60 \mathrm{GeV}$, the signal region for this analysis has been defined with $\#_{T}>50 \mathrm{GeV}$. This choice is also motivated by the fact that the expected SM background is already at a very low level when requiring $E_{T}>50 \mathrm{GeV}$. Further cuts have as well been considered but are not used in this analysis for the same reason.

Based on the above described considerations, the signal region for this analysis has been defined as follows: 


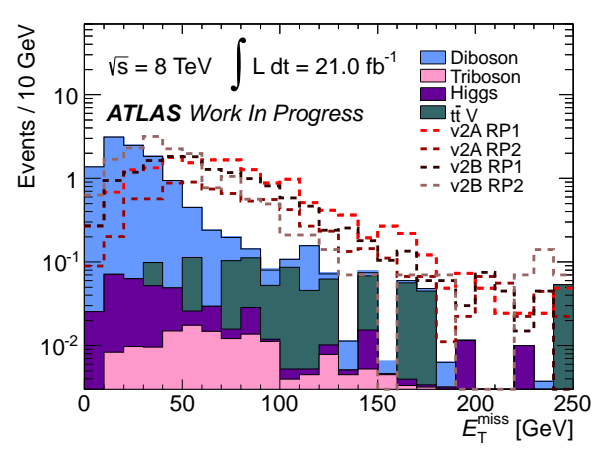

(a) $E_{T}$ spectrum

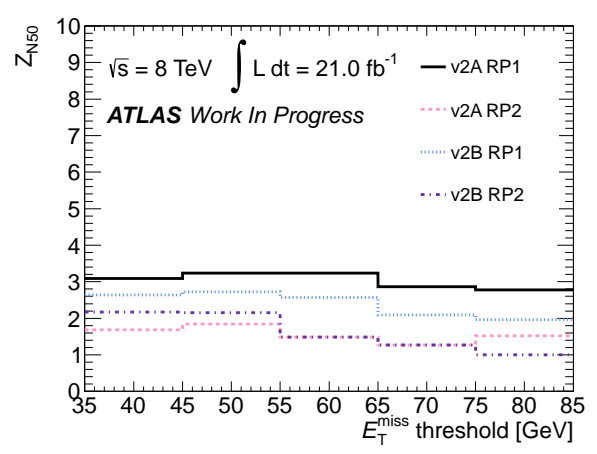

(b) expected significance

Figure 6.12: Distribution of a variable with a possible relevance to the expected significance. Figure (a) shows the distribution of the missing transverse energy after requiring at least four reconstructed signal leptons and no reconstructed $\mathrm{Z}$ candidate in the event. Figure (b) shows the expected significance as a function of $E_{T}$ threshold, where an uncertainty of $50 \%$ on the background expectation is taken into account.

- There are four or more signal leptons in the event, $n_{L, \text { signal }} \geq 4$.

- There is no additional 'loose' lepton in the event, $n_{L, \text { loose }}=0$.

- There is no leptonic $\mathrm{Z}$ candidate in the event, $\mathrm{Z}$ veto.

- The amount of missing transverse energy in the event exceeds $50 \mathrm{GeV}, \mathbb{E}_{T}>50 \mathrm{GeV}$.

Using MC simulations for the SM background, the selection efficiency, the expected number of signal events and the expected significance for both grids in this signal region is shown in Figure 6.13, where a bilinear interpolation is used to calculate the numbers in between the grid points that were defined above. The total systematic uncertainty on the expected number of signal events is shown in Figure 6.14, where in addition to the theory uncertainty on the cross section all systematics as described in the next section are considered. Roughly, the average total systematic uncertainty lies between $10 \%$ and $20 \%$, where in regions with small mass splittings, due to the poor acceptance, the total uncertainty can be significantly larger, mainly due to an increased uncertainty from the MC statistics.

\subsection{Estimation of the SM Background}

While the irreducible SM background is estimated from MC simulations, the number of events with four or more leptons, of which at least one lepton is not a prompt lepton, is estimated using a data driven technique. In the first part of this section, the SM background in the signal region as expected from MC simulations is described, whereas in the second part the estimation of the fake-lepton background is explained.

\subsubsection{Irreducible Background Processes}

As the dominant source of irreducible background, the production of two top quarks in association with a $Z$ boson or two $W$ bosons is considered in the estimation. These events contribute 


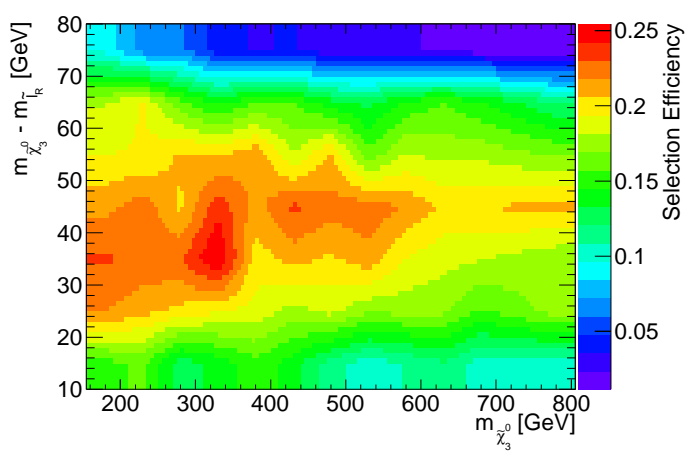

(a) v2A: Selection Efficiency

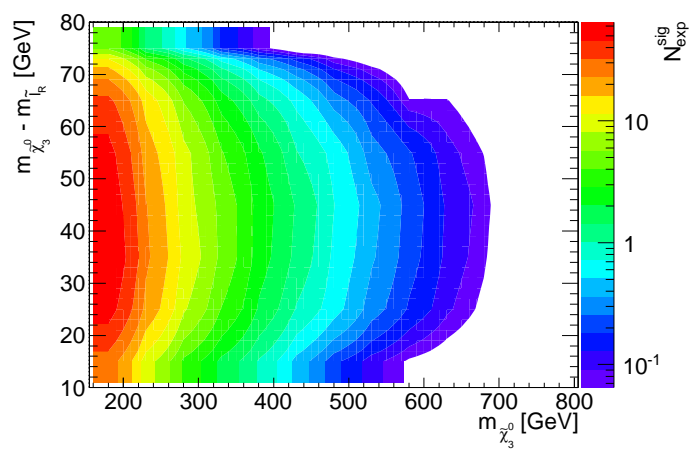

(c) v2A: expected number of signal events

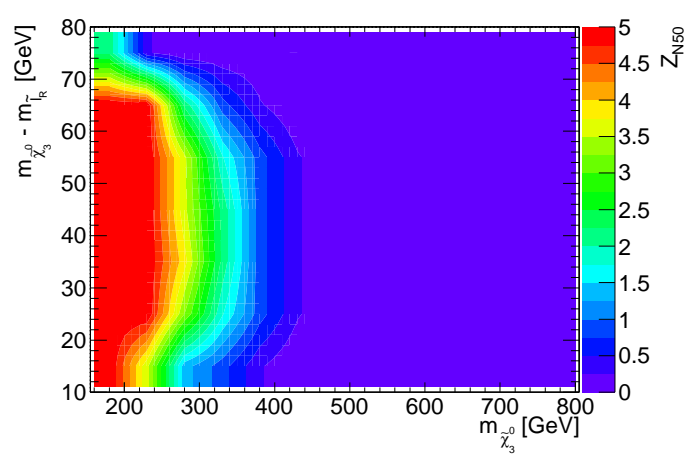

(e) v2A: expected significance

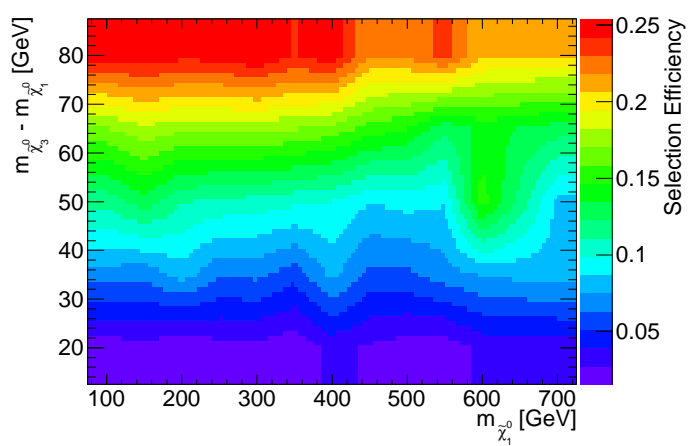

(b) v2B: Selection Efficiency

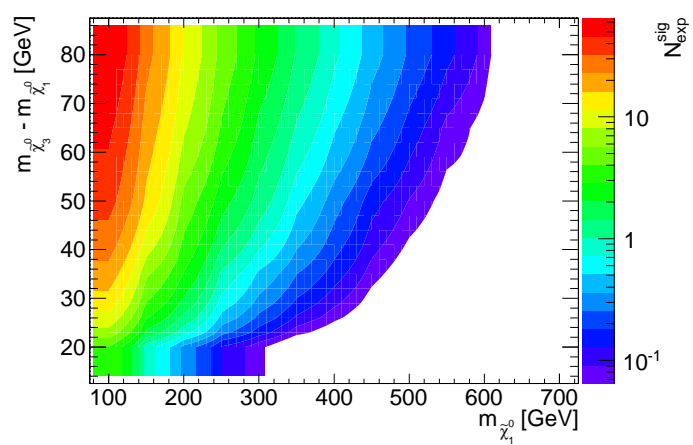

(d) v2B: expected number of signal events

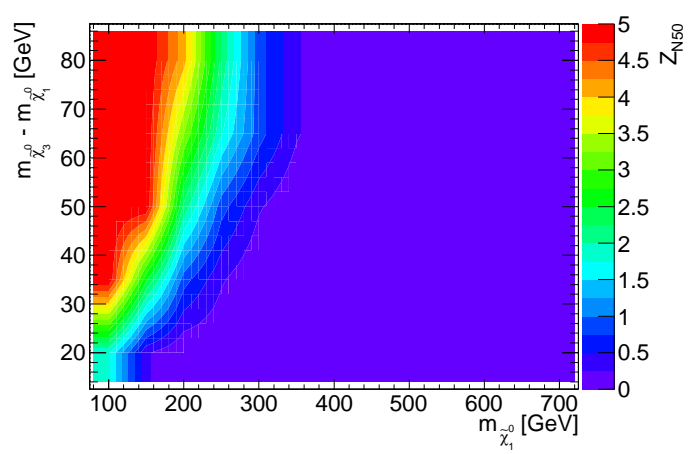

(f) v2B: expected significance

Figure 6.13: The selection efficiency for the signal region (top), the expected number of signal events (middle) and expected significance $Z_{N}$ (bottom) across the simplified signal models for $\tilde{\chi}_{3}^{0}-\tilde{\chi}_{2}^{0}$ production in the signal region. For the grid points where the signal is much larger than the SM background, $Z_{N}$ is set by hand to a value of 5 . Bilinear interpolation is used to calculate the values in between the discrete grid points.

because there is a considerable chance that both top quarks and the additional gauge bosons decay into light leptons. In addition, there is real missing transverse energy in such events.

The other important background for this analysis is the associate production of $Z$ bosons, where both of the $Z$ bosons decay into light leptons. While for all signal scenarios that are 


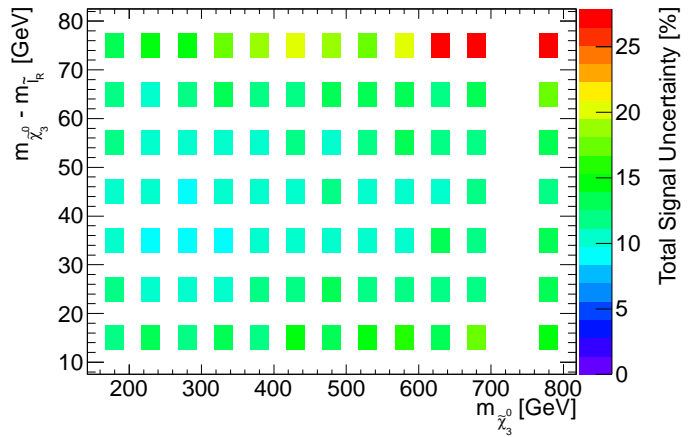

(a) v2A: systematic uncertainty

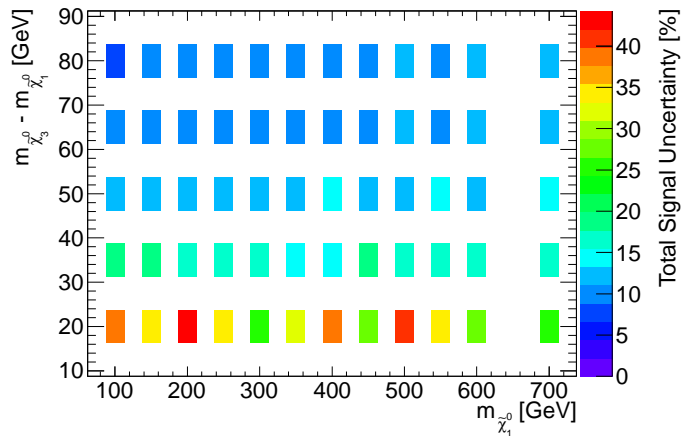

(b) v2B: systematic uncertainty

Figure 6.14: The total systematic uncertainty on the expected number of signal events. For most points, the signal uncertainty is roughly $10 \%-20 \%$. Points with a small acceptance and efficiency exhibit a larger uncertainty, mainly due to the small statistics after selection in the MC samples.

considered in this analysis the total cross section for the associate production of two $Z$ bosons is larger than the signal cross section by at least two orders of magnitude, the probability to have four prompt charged leptons in a genuine $Z Z$ event is only $0.5 \%$, roughly. If in addition both $Z$ bosons are required to be far off the nominal $Z$ mass, and if a considerable amount of missing transverse energy is required, a reasonable sensitivity to the new physics scenarios can be achieved.

Four prompt leptons in the final state are also expected in events where three electroweak gauge bosons are produced; in particular the production of one $Z$ boson in association with two $W$ bosons or two $Z$ bosons, where real missing transverse energy due to neutrinos in the final state is expected.

Also, events in which a Higgs boson is produced can contribute to the irreducible background, if the Higgs decays into a pair of $Z / Z^{*}$ bosons; in particular if the Higgs boson is produced in association with another $Z$ boson. The signal models that are considered in this study do not make any assumptions about the SUSY Higgs sector. A SM-like Higgs boson is therefore taken into account in the background estimation; the production of Higgs bosons is not considered as a signal in this analysis. While it is yet unclear if the boson that was discovered at the ATLAS experiment and the CMS experiment in 2012 is really a (the) Higgs boson, the observations made in the searches for the SM Higgs in final states with four leptons justify the consideration of a SM-like Higgs boson in the background estimation for the presented analysis.

In all background processes, the bosons may be off shell, and in addition to $Z$ bosons, the production of $\gamma^{*}$ is as well taken into account.

\subsubsection{Reducible Background Processes}

The dominant contribution to the reducible background is expected from processes with three prompt leptons and one additional fake lepton in the final state. Sources for fake leptons are both heavy flavour and light flavour jets, as well as electrons that are produced in pairs from photons that are radiated in the initial or final state. While fake leptons can in fact be real leptons, they do not originate from the hard interaction and do therefore not qualify as signal leptons for the purpose of this analysis (cf. chapter 5). These are thus considered as fake leptons, 
here.

The production of a $W$ boson in association with a $Z / \gamma^{*}$, as well as the associate production of three $W$ bosons and the production of a top quark pair in association with a $W$ boson, are taken into account.

While the SM background from events with three real leptons and one or more fake leptons is already expected to be small, the contribution from events with two prompt leptons and two or more fake leptons is yet expected to be much smaller. The production of top quark pairs, two $W$ bosons, a $Z / \gamma^{*}$ and also single top production, where in addition to the top quark a $W$ boson emerges from the hard interaction, are sources for this background.

In events with one real lepton, such as the production of a single top quark in the s- or t-channel, as well as the production of a single $W$ boson, four or more signal leptons may be reconstructed if there are three or more non-prompt leptons in the event. The contribution of these processes to the signal region has been found to be negligible.

\subsubsection{Background Expectations from MC}

The number of events in the signal region as expected from SM MC is summarised in Table 6.3, where for completeness also the reducible backgrounds are included. The following sources of systematic uncertainties on the expectation value of the SM background have been considered for the contributions that are estimated from MC, where the numbers for the experimental systematics are provided by the respective ATLAS combined performance groups:

- Jet Energy: Uncertainties due to the Jet Energy Scale (JES) are considered in both the calculation of the relevant jet properties as well as in the calculation of the related components of the missing transverse energy. The uncertainty due to the Jet Energy Resolution (JER) is determined by smearing the transverse momentum of each jet.

- Electron Energy: The uncertainty due to the electron energy scale (EES) and the electron energy resolution (EER) are estimated from an analysis of events with massive gauge bosons for high $p_{T}$ electrons, and events with a $J / \psi$ candidate for low $p_{T}$ electrons. As for jets, the uncertainty is taken into account for the calculation of the electron properties and the related components of the missing transverse energy.

- Muon Energy: The uncertainties on the muon energy/momentum are calculated by smearing the muon transverse momentum. Two contributions to the uncertainty from the ID track (MID) and the MS track (MMS) are taken into account. As in the case of jets and electrons, also this uncertainty is taken into account for the calculation of the missing transverse energy.

- Tau Energy: The uncertainties on the tau energy scale are considered as a function of the tau $p_{T}$, the tau $\eta$ and the number of tracks that are associated to the reconstructed tau candidate. Although this analysis does not make any requirements on taus in the final state, the uncertainty on the tau energy scale and the tau energy resolution has a small impact on the number of events in the signal region due to the overlap removal, where taus are considered.

- Lepton Efficiency Scale Factors: An uncertainty is on the scale factors that are used to correct the MC for the differences in the lepton reconstruction efficiencies is taken into account by varying these scale factors accordingly (MEFF, ESF). Since the uncertainties on the efficiency scale factors are calculated using signal leptons in the signal region only, 
uncertainties on tau reconstruction efficiency scale factors are not taken into account in this analysis.

- MET: An uncertainty is applied on the resolution and the energy scale of the soft term in the missing transverse energy.

- Luminosity: The uncertainty on the luminosity (3.6\%) is considered for scaling the MC to data.

- Trigger: As explained above, the average trigger efficiency for events with four leptons in the final state is large, such that no correction is applied to the MC. A conservative uncertainty of $5 \%$ is attributed to the average efficiency in MC.

- Cross section: The cross sections for some of the SM background processes have not yet been measured. For the production of a top quark pair in association with a massive gauge boson, an uncertainty of $30 \%$ is used, whereas for the production of the triboson samples an uncertainty of $100 \%$ is applied. The uncertainty on the diboson samples are $5 \%$ for $Z Z$ production and $7 \%$ for WZ production.

- Generator: The expected number of background events may depend on the generator that is used to calculate the predictions. For the $Z Z$ background, an uncertainty is derived from the comparison of the number of events predicted by SHERPA and the number of events predicted by POWHEG, which yields an uncertainty of $78 \%$. A comparison of Alpgen and MadGraph is performed to derive an uncertainty on the $t \bar{t} Z$ production; this is estimated to $15 \%$ in the signal region.

- PDF: Uncertainties on the parton distribution functions are taken into account for the $Z Z$ and the $W Z$ samples. For other samples, this is already included in the cross section uncertainties.

- MC statistics: The number of unscaled (raw) events in the MC that pass the full signal selection is usually quite small. This is taken into account by applying a proper contribution to the total uncertainty on the expectation value.

\subsubsection{Estimation of the Reducible Background}

The estimation of the reducible background is performed using a semi data driven method, relying on some approximations. A more complete description of the method and all measurements that have been performed for its application in this analysis can be found in [147].

The reducible background consists of processes with less than four prompt leptons and one or more fake leptons and the method used for the estimation of the reducible background in the signal region exploits differences in the quality and isolation of reconstructed fake leptons compared to reconstructed prompt leptons on a statistical basis. In this context, reconstructed leptons are classified as 'loose' leptons and 'tight' leptons. A 'tight' lepton is a signal lepton as defined in section 6.3.4. A 'loose' electron is defined as a baseline electron that is not removed in the overlap removal, but which fails the isolation requirement or the tight++ or impact parameter requirements. A 'loose' muon is defined as a baseline muon that is not removed in the overlap removal but which fail the isolation or impact parameter requirements. Note that the definition of 'loose' and 'tight' leptons is mutually exclusive. 
In the following, the index $L(T)$ indicates a 'loose' ('tight') and the index $R(F)$ indicates a real (fake) lepton. Let $n_{R}\left(n_{F}\right)$ be the number of reconstructed 'loose' or 'tight' real (fake) leptons, then the real reconstruction efficiency $r$ and the fake rate $f$ is defined as

$$
\begin{aligned}
& r=\lim _{n_{R} \rightarrow \infty} \frac{n_{R}^{T}}{n_{R}}, \\
& f=\lim _{n_{F} \rightarrow \infty} \frac{n_{F}^{T}}{n_{F}},
\end{aligned}
$$

where as in the case of trigger efficiencies (cf. chapter 5) a possible dependence of both $r$ and $f$ on one or more properties of the leptons, such as the lepton flavour, transverse momentum or pseudorapidity, is implied. In the following, the notation $\bar{f}=1-f$ and $\bar{r}=1-r$ is used.

Due to the limited number of events with four or more reconstructed leptons, the estimation of the reducible background as outlined in the following relies on some approximations. First, since the number of events with three or more fake leptons is expected to be very small, such events are not considered in the following. Second, the probability that a real lepton is reconstructed as a signal lepton, is usually very high, such that events with three or more 'loose' leptons are also not considered.

The following derivation of the formula that is used to calculate the expectation value for the number of events with one or more fake leptons is therefore performed using a simplified

\begin{tabular}{lr}
\hline Process & $N_{M C}^{S R} / 20.7 \mathrm{fb}^{-1}$ \\
\hline $\mathrm{ZZ}$ & $0.60_{-0.39}^{+0.39}$ \\
$\mathrm{WZ}$ & $0.06_{-0.06}^{+0.06}$ \\
$\mathrm{WW}$ & $0.00_{-0.00}^{+0.00}$ \\
ZWW & $0.12_{-0.12}^{+0.12}$ \\
$\mathrm{ZZZ}$ & $0.00_{-0.00}^{+0.00}$ \\
$\mathrm{WWW}$ & $0.00_{-0.00}^{+0.00}$ \\
Higgs & $0.26_{-0.07}^{+0.07}$ \\
$\mathrm{t} \overline{\mathrm{t}}$ & $0.04_{-0.04}^{+0.15}$ \\
$\mathrm{t} \overline{\mathrm{tZ}}$ & $0.77_{-0.35}^{+0.35}$ \\
$\mathrm{t} \overline{\mathrm{t}} \mathrm{t}$ & $0.01_{-0.01}^{+0.01}$ \\
$\mathrm{t} \overline{\mathrm{t}} \mathrm{WW}$ & $0.01_{-0.01}^{+0.01}$ \\
$\mathrm{DY} / \mathrm{Z} / \mathrm{W}+$ jets and single t & $0.00_{-0.00}^{+0.00}$ \\
\hline Total SM (MC) & $1.87_{-0.72}^{+0.72}$ \\
\hline
\end{tabular}

Table 6.3: The expected number of signal events for different SM processes. The estimations are obtained using MC only. The estimation of backgrounds with fake leptons is replaced by an estimate from data for the final results. These numbers have been used to define and freeze the signal region. 
notation with only two leptons. If the above approximations are taken into account, the final result of this simplified calculation does not differ from the result that is obtained by using a matrix with four leptons, but the calculation is significantly longer.

In the following, let $N_{R R}$ be the number of events with two real leptons, $N_{R F}\left(N_{F R}\right)$ the number of events with one real and one fake lepton, where the real lepton is the leading (subleading) lepton (in terms of $p_{T}$ ), and $N_{F F}$ the number of events with two fake leptons. The same notation is used with indices $T$ and $L$ for 'tight' and 'loose' leptons. Then, the following equation holds, where the index 1 denotes the leading lepton and the index 2 the subleading lepton:

$$
\left(\begin{array}{c}
N_{T T} \\
N_{T L} \\
N_{L T} \\
N_{L L}
\end{array}\right)=\left(\begin{array}{cccc}
r_{1} r_{2} & r_{1} f_{2} & f_{1} r_{2} & f_{1} f_{2} \\
r_{1} \bar{r}_{2} & r_{1} \bar{f}_{2} & f_{1} \bar{r}_{2} & f_{1} \bar{f}_{2} \\
\bar{r}_{1} r_{2} & \bar{r}_{1} f_{2} & \bar{f}_{1} r_{2} & \bar{f}_{1} f_{2} \\
\bar{r}_{1} \bar{r}_{2} & \bar{r}_{1} \bar{f}_{2} & \bar{f}_{1} \bar{r}_{2} & \bar{f}_{1} \bar{f}_{2}
\end{array}\right) \cdot\left(\begin{array}{c}
N_{R R} \\
N_{R F} \\
N_{F R} \\
N_{F F}
\end{array}\right)
$$

The number of events with two tight leptons where at least one lepton is a fake lepton is then given by

$$
N_{\overline{T T}}^{\geq 1 \text { fake }}=N_{T T}-r_{1} r_{2} N_{R R}
$$

which can easily be calculated by multiplying each row in equation (6.19) with a well chosen term and subtracting the second and third row from the sum of the first and fourth row:

$$
\left(\begin{array}{c}
N_{T T} \\
N_{T L} \frac{f_{2}}{\bar{f}_{2}} \\
N_{L T} \frac{f_{1}}{\bar{f}_{1}} \\
N_{L L} \frac{f_{1}}{\bar{f}_{1}} \frac{\frac{f_{2}}{\bar{f}_{2}}}{f_{2}}
\end{array}\right)=\left(\begin{array}{cccc}
r_{1} r_{2} & r_{1} f_{2} & f_{1} r_{2} & f_{1} f_{2} \\
r_{1} \bar{r}_{2} \frac{f_{2}}{\bar{f}_{2}} & r_{1} f_{2} & f_{1} \bar{r}_{2} \frac{f_{2}}{\bar{f}_{2}} & f_{1} f_{2} \\
\bar{r}_{1} r_{2} \frac{f_{1}}{\bar{f}_{1}} & \bar{r}_{1} f_{2} \frac{f_{1}}{\bar{f}_{1}} & f_{1} r_{2} & f_{1} f_{2} \\
\bar{r}_{1} \bar{r}_{2} \frac{f_{1}}{\bar{f}_{1}} \frac{\underline{f}_{2}}{\bar{f}_{2}} & \bar{r}_{1} f_{2} \frac{f_{1}^{1}}{\bar{f}_{1}} & f_{1} \bar{r}_{2} \frac{f_{2}}{\bar{f}_{2}} & f_{1} f_{2}
\end{array}\right) \cdot\left(\begin{array}{c}
N_{R R} \\
N_{R F} \\
N_{F R} \\
N_{F F}
\end{array}\right),
$$

such that

$$
\begin{aligned}
N_{\overline{T T}}^{\geq 1 \text { fake }} & =N_{T T}-r_{1} r_{2} N_{R R} \\
& =\left(N_{T L}-r_{1} \bar{r}_{2} N_{R R}\right) \frac{f_{2}}{\bar{f}_{2}}+\left(N_{L T}-\bar{r}_{1} r_{2} N_{R R}\right) \frac{f_{1}}{\bar{f}_{1}}-\left(N_{L L}-\bar{r}_{1} \bar{r}_{2} N_{R R}\right) \frac{f_{1}}{\bar{f}_{1}} \frac{f_{2}}{\bar{f}_{2}}(6
\end{aligned}
$$

In the case of four leptons, the number of events with four real leptons, where one or two are reconstructed as 'loose' leptons is estimated from MC, such that

$$
N_{\mathrm{BKG}, 4 \mathrm{~T}}^{\mathrm{RED}}=\left(N_{3 T 1 L}^{\text {data }}-N_{3 T 1 L}^{\mathrm{MC}, \mathrm{IRR}}\right) \cdot F_{i}-\left(N_{2 T 2 L}^{\text {data }}-N_{2 T 2 L}^{\mathrm{MC}, \mathrm{IRR}}\right) \cdot F_{j} \cdot F_{k},
$$

where $F_{i}=\frac{f_{i}}{f_{i}}$ and in practice the calculation is performed on an event-by-event basis, taking into account the dependence of the fake ratios $F_{i}$ on the lepton properties; each event is assigned a weight, and the number of events with one or two fakes in the four lepton signal region is given by the sum of those weights. Also, a correction to the formula is applied to account for events with more than four leptons, in particular events with two 'tight' leptons and three or more 'loose' leptons.

The most important input for this method is a measurement of the fake ratios $F_{i}$. As noted above, the fake ratio depends on the lepton flavour, as well as the origin of the fake leptons and several of the lepton properties. For instance, the fake ratio for leptons from heavy flavour jets depends on the transverse momentum of the b-quark that emerges from the hard interaction. 
This can however not be used as a parameter, since the b-quark momentum is unknown. In effect, the average fake ratio for muons from heavy flavour jets in top quark events is different from the fake ratio for muons from heavy flavour jets in QCD multijet events.

Since it is difficult to accurately measure the fake ratios for all processes that have to be considered, a semi-data driven approach is followed for the estimation of these. In a first step, MC samples have been used to determine the fake ratios for the different processes in MC. For muons, two different fake ratios are considered, while for electrons four different fake ratios are considered, depending on the origin of the fake lepton and the underlying hard process. The results are summarised in Table 6.4, where an average fake ratio is given. The production of top quarks and the production of massive electroweak gauge bosons are considered as the two dominant underlying hard interactions, and heavy flavour jets are considered as an origin for fake electrons and fake muons. Light flavour jets can in principle as well contribute to the number of fake leptons, but these contributions are small compared to the heavy flavour fraction and are therefore absorbed in it. For electrons, also the conversion of a photon is considered as an origin for fake electrons. The fake ratios are measured as a function of the lepton $p_{T}$ and lepton $\eta$.

\begin{tabular}{l|l|c|c|c} 
Fake Type & Hard Process & Average Fake Ratio & Scale Factor & Fraction in SR \\
\hline e from HF jets & boson & $0.110 \pm 0.008$ & $0.73 \pm 0.06$ & 0.053 \\
& top & $0.047 \pm 0.001$ & $0.73 \pm 0.06$ & 0.832 \\
e from conversion & boson & $0.190 \pm 0.008$ & $1.27 \pm 0.31$ & 0.056 \\
& top & $0.075 \pm 0.006$ & $1.27 \pm 0.31$ & 0.059 \\
$\mu$ from HF jets & boson & $0.240 \pm 0.015$ & $0.83 \pm 0.04$ & 0.054 \\
& top & $0.153 \pm 0.003$ & $0.83 \pm 0.04$ & 0.945
\end{tabular}

Table 6.4: The average fake ratios as measured in MC, the data/MC scale factors and the fraction of events in the signal region, that are used as an input for the data driven background estimation. For more information on these numbers, see [147].

In a second step scale factors for the fake ratios have been determined by comparing well defined data samples with MC for processes that dominate the region studied in data. It is assumed that the scale factors do not depend on the underlying hard process, but only on the origin of the fake leptons.

The fake ratios and the scale factors for electrons and muons from heavy flavour jets as measured in dedicated regions in data and MC are shown in Figures 6.15 and 6.16. $\mathrm{A} b \bar{b}$ Tag \& Probe method has been used to select the data samples, which are rich in $b \bar{b}$ jet pairs [147].

In a third step, to calculate the fake ratios that apply to the signal region, the composition of the fake background in the signal region is estimated from $\mathrm{MC}$, and the fake ratio for each hard process and fake lepton origin is multiplied with the scale factor and the corresponding fraction $R^{\text {process,origin }}$ of total events in the signal region, such that the fake ratio in the signal region is given by

$$
F_{i}=\sum_{\text {process,origin }} \frac{F_{i}^{\text {data,origin }}}{F_{i}^{\mathrm{MC}, \text { origin }}} \cdot F_{i}^{\mathrm{MC}, \text { origin,process }} \cdot R^{\text {process,origin }} .
$$

The following systematic uncertainties are considered for the prediction of the reducible background:

- Fake Ratio: The fake rates depend on several event and object properties, and these dependencies can not be fully covered by a fine binning of the fake rates. Therefore, a 


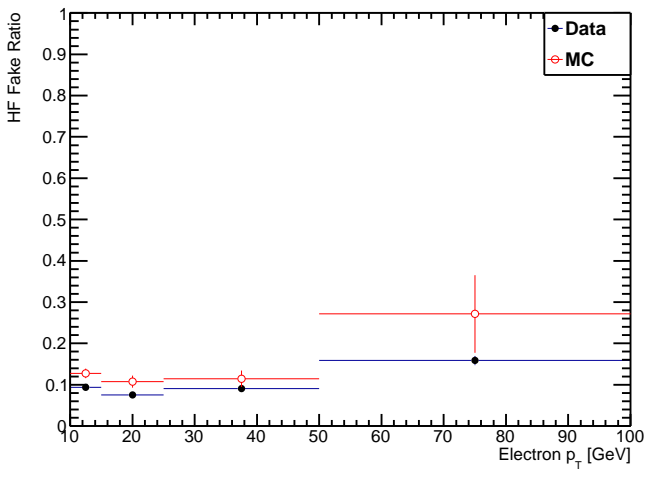

(a) Fake Ratio, electron $p_{T}$

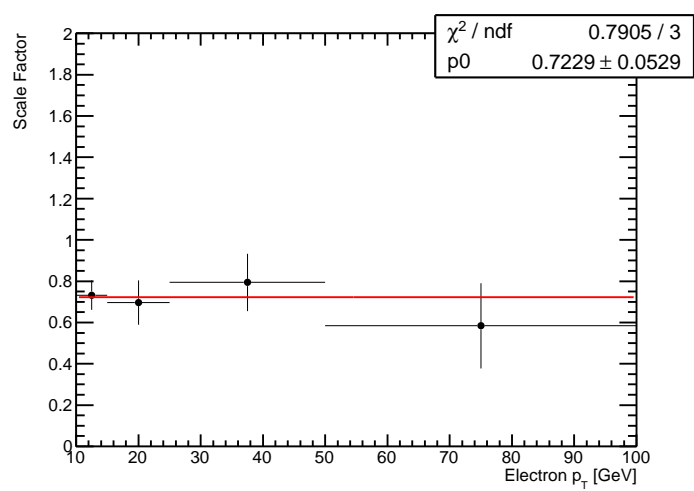

(c) Scale Factor, electron $p_{T}$

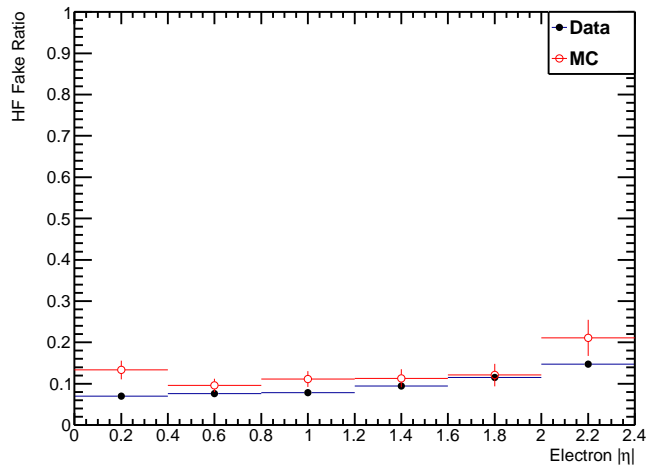

(b) Fake Ratio, electron $\eta$

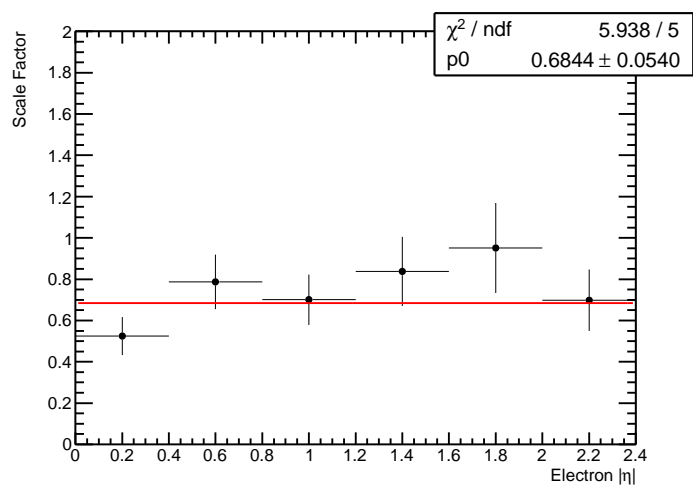

(d) Scale Factor, electron $\eta$

Figure 6.15: The fake ratios and scale factors for electrons from heavy flavour jets, measured as a function of the electron $p_{T}$ and the electron $\eta$ [147].

total uncertainty of $10 \%$ is applied to the electron fake ratios, and a total uncertainty of $30 \%$ is applied to the muon fake ratios.

- Scale Factors: For fakes from heavy flavour jets, a total uncertainty of $4 \%$ is applied for the muon fake ratio scale factors and $6 \%$ for electron fake ratio scale factors. For electrons from conversion, a total uncertainty of $22 \%$ is applied.

- Fraction: The fraction of events with fake leptons from a particular source are not known very precisely. Therefore, an uncertainty of $100 \%$ is applied on these fractions. 


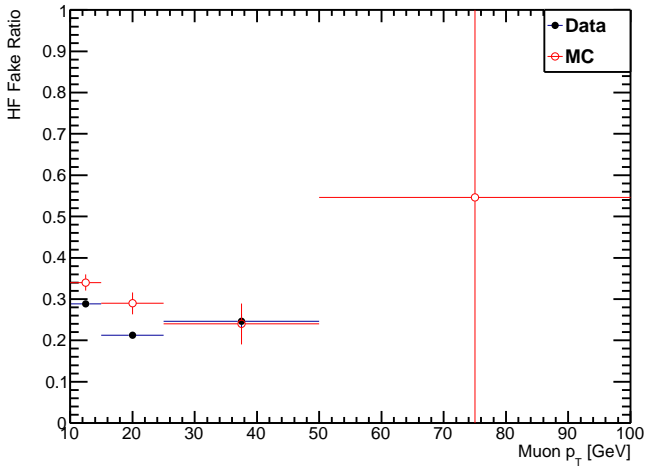

(a) Fake Ratio, muon $p_{T}$

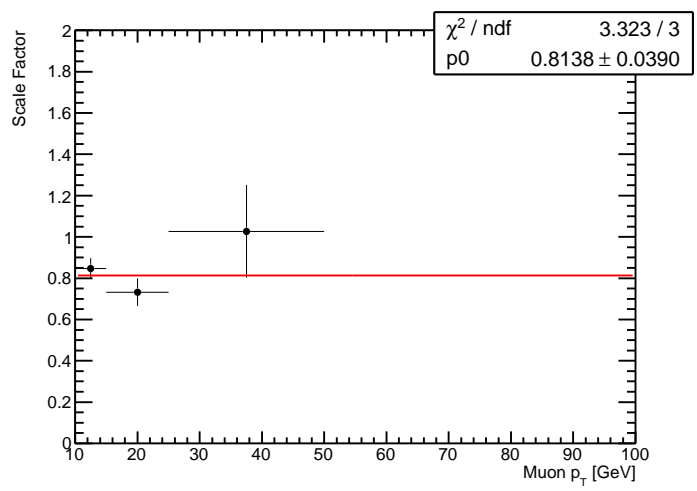

(c) Scale Factor, muon $p_{T}$

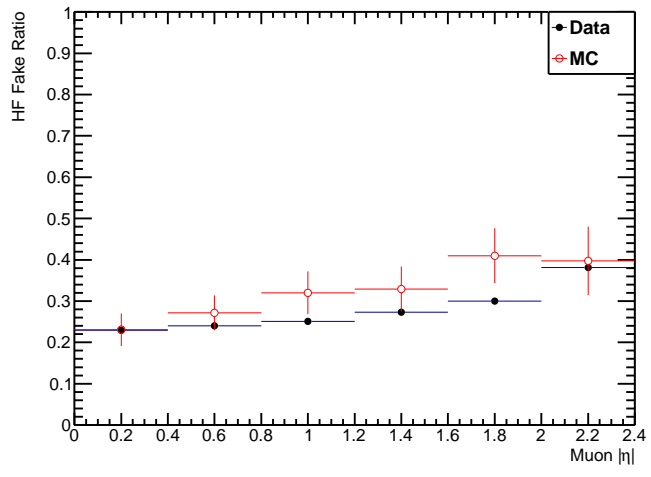

(b) Fake Ratio, muon $\eta$

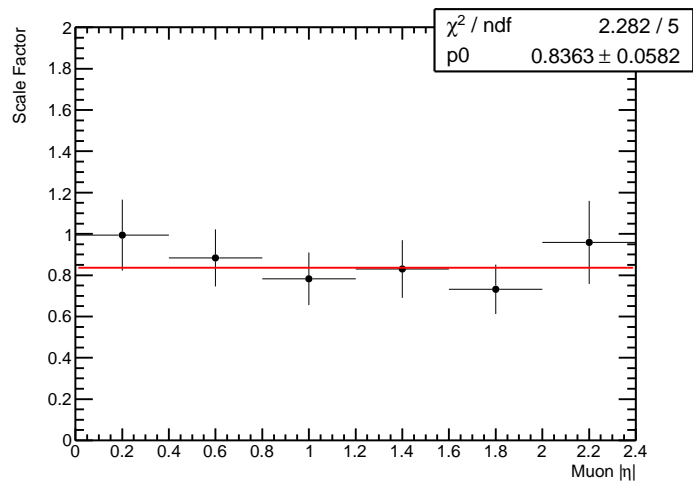

(d) Scale Factor, muon $\eta$

Figure 6.16: The fake ratios and scale factors for muons from heavy flavour jets, measured as a function of the muon $p_{T}$ and the muon $\eta[147]$.

\subsubsection{Validation of the Background Modelling}

The background model has been validated using two control regions. The first control region is very similar to the signal region. Four or more light signal leptons are required, events containing lepton combinations that make up a $\mathrm{Z}$ candidate are vetoed. In order to avoid overlap with the signal region, the amount of missing transverse energy is required to be less than $50 \mathrm{GeV}$. This region is referred to as VRnoZ. A contamination of the validation region with signal events is not taken into account.

Since in this region only a few events are expected in data, and the contamination with signal events may be significant, a second control region is defined. Four or more light signal leptons are required, and in addition a leptonic $\mathrm{Z}$ candidate is demanded. The amount of missing transverse energy is still required to be less than $50 \mathrm{GeV}$ to ensure orthogonality with other signal regions that are used in the more comprehensive analysis [147]. This region is referred to as VRZ. A contamination of signal events is as well not considered in this region, where for the $\tilde{\chi}_{2}^{0}-\tilde{\chi}_{3}^{0}$ models it is expected to be negligible, anyways.

The predicted number of events according to the SM and the observed number of events in data are summarised in Table 6.5. The agreement between data and predictions is reasonable. A comparison of several distributions is shown in Figure 6.17 for VRnoZ and in Figure 6.18 for 
VRZ. Both the absolute number of events as well as the shape of the distributions are in good agreement, although the explanatory power of VRnoZ is limited by the small statistics in that region, and an underfluctuation in data is observed compared to the SM expectation. More plots for the validation of the background model can be found in [147].

\begin{tabular}{lrr}
\hline Process & VRnoZ & VRZ \\
\hline ZZ & $7.2_{-3.6}^{+3.6}$ & $167_{-38}^{+38}$ \\
ZWW & $0.03_{-0.03}^{+0.03}$ & $0.35_{-0.35}^{+0.35}$ \\
ZZZ & $<0.01_{-0.00}^{+0.00}$ & $0.02_{-0.02}^{+0.02}$ \\
Higgs & $0.17_{-0.05}^{+0.05}$ & $4.5_{-0.9}^{+0.9}$ \\
$\mathrm{t} \bar{t} \mathrm{C}$ & $0_{-0.00}^{+0.05}$ & $1.5_{-0.6}^{+0.7}$ \\
$\mathrm{t} \bar{t} \mathrm{WW}$ & $<0.01_{-0.00}^{+0.00}$ & $<0.01_{-0.00}^{+0.00}$ \\
Irreducible SM & $7.4_{-3.6}^{+3.6}$ & $173_{-39}^{+39}$ \\
Reducible SM & $0.3_{-0.3}^{+0.7}$ & $2.0_{-2.0}^{+2.6}$ \\
\hline Total SM & $7.7_{-3.4}^{+3.4}$ & $175_{-37}^{+37}$ \\
Data & 3 & 201 \\
\hline CL & 0.10 & 0.51 \\
\hline
\end{tabular}

Table 6.5: SM expectation and observed number of events in the two background model validation regions. The agreement between predictions and the observation is reasonable and the background model can be assumed to be valid. 


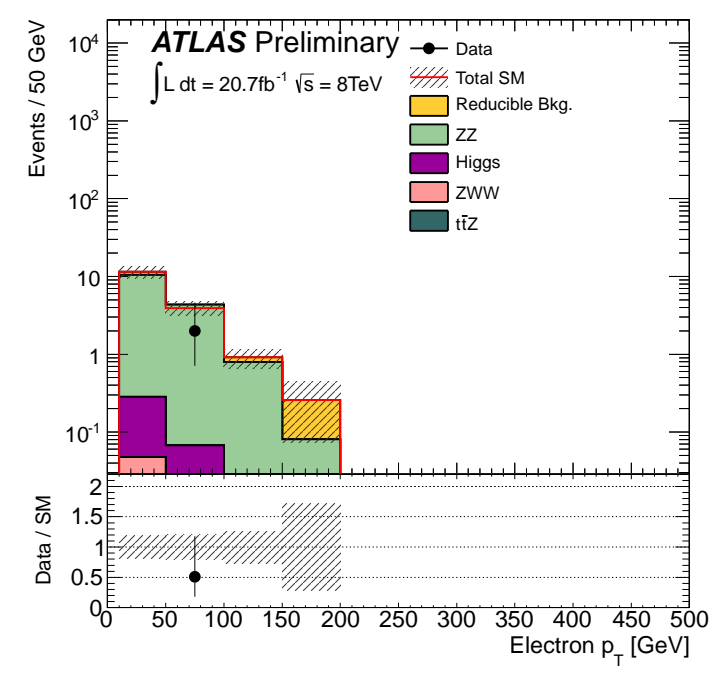

(a)

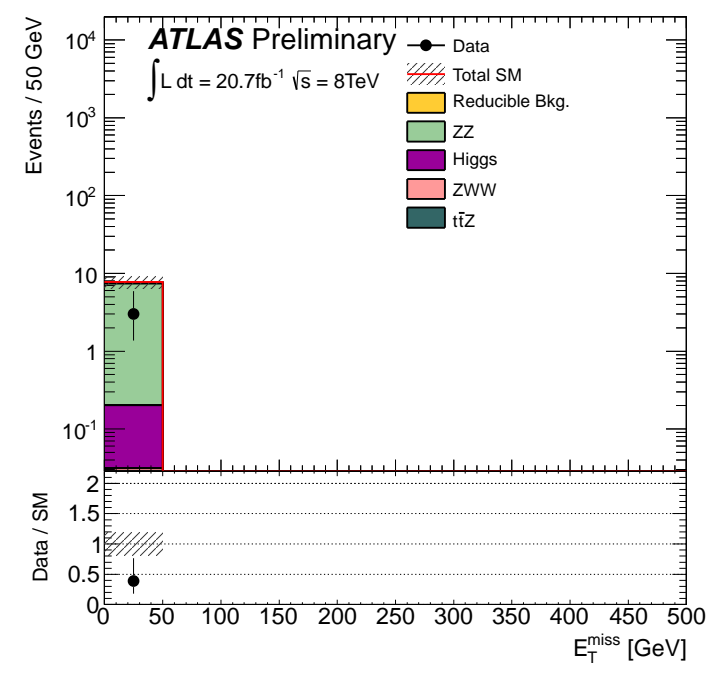

(c)

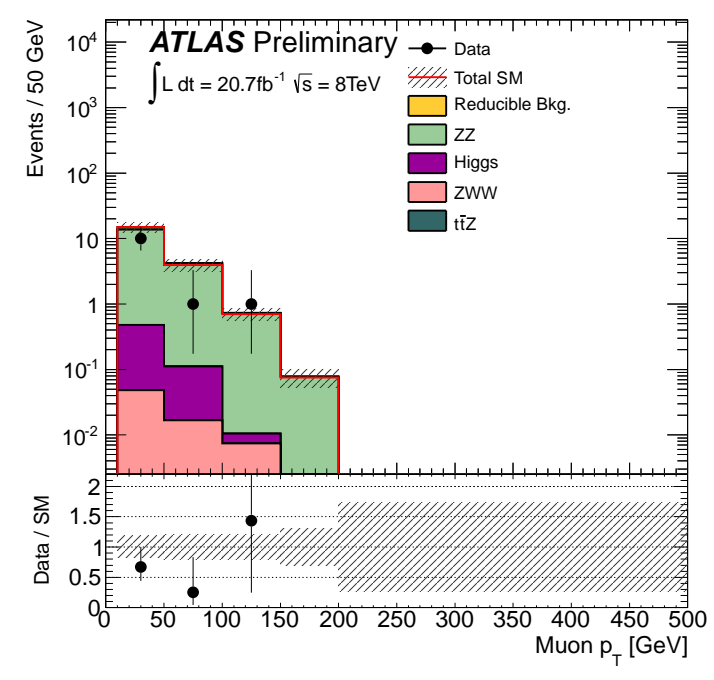

(b)

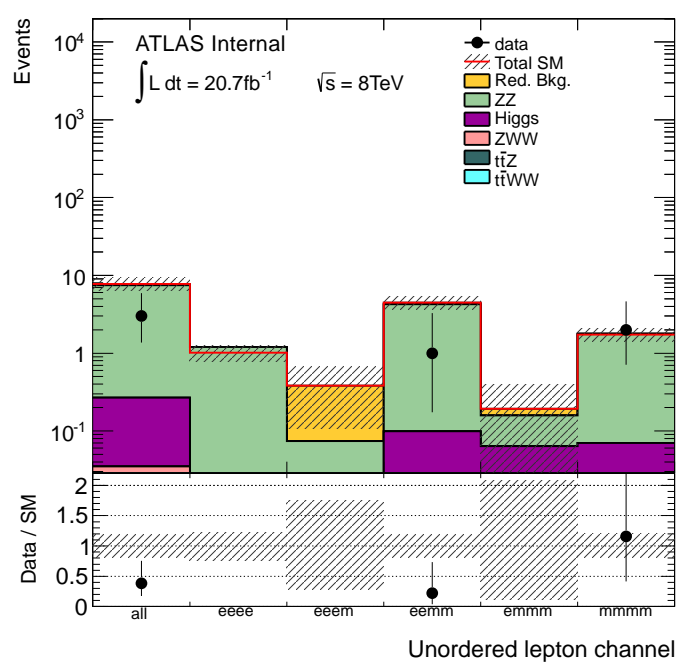

(d)

Figure 6.17: Distributions in the $\mathrm{Z}$ veto region that is used to validate the background modelling, VRnoZ [147]. 


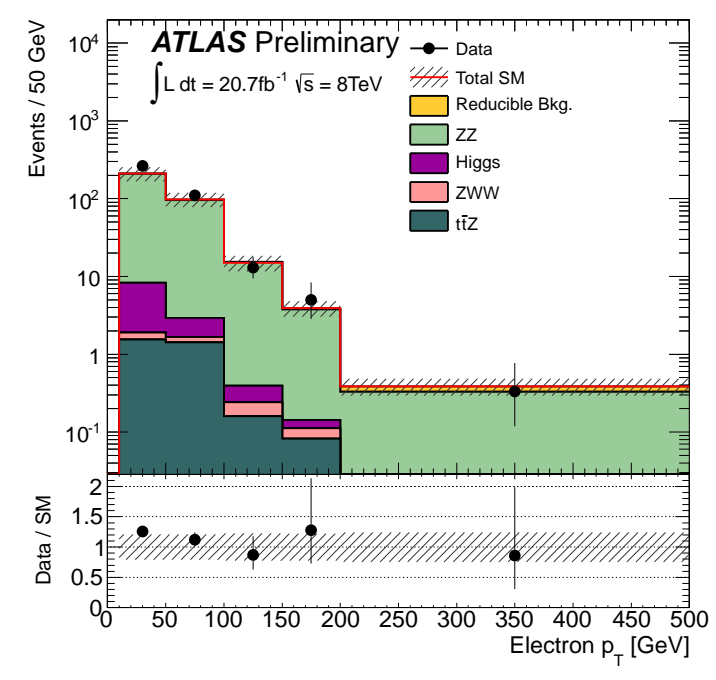

(a)

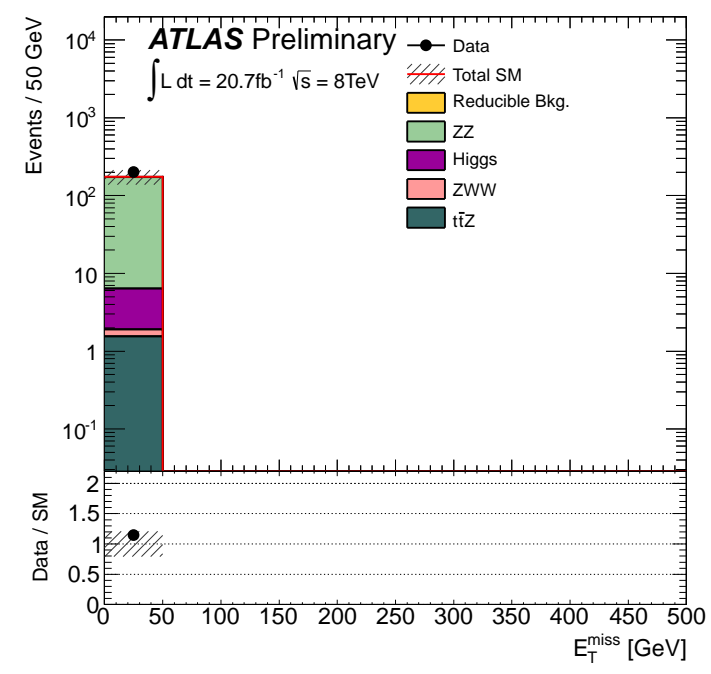

(c)

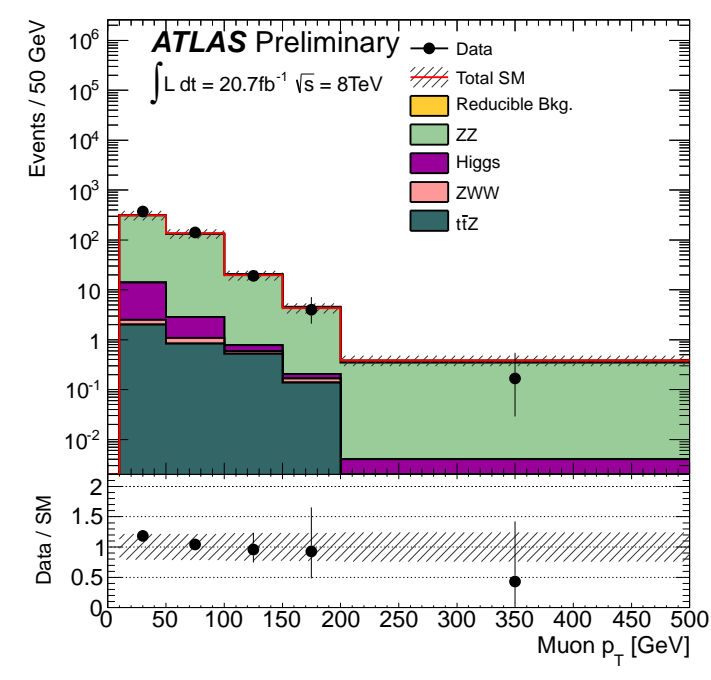

(b)

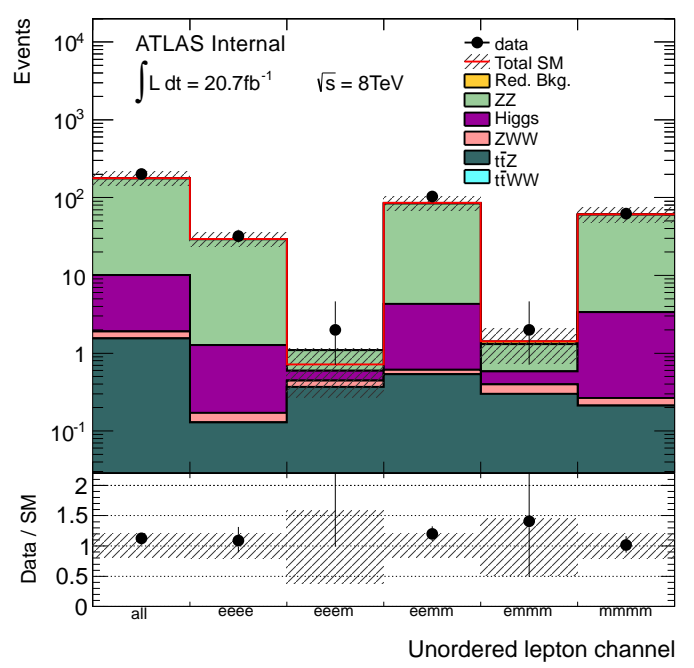

(d)

Figure 6.18: Distributions in the $\mathrm{Z}$ request region that is used to validate the background modelling, VRZ [147]. 


\subsection{Analysis Results}

The expected SM background in the SR is shown with the number of observed events in Table 6.6. A total of $1.7 \pm 0.8$ events is expected and 2 events are observed in the signal region, which yields a p-value for the background-only hypothesis of $p_{0}=0.29$, reflecting a good agreement between the SM expectation and the observed data. The data driven estimate for the reducible background is negative due to underfluctuations in data and is therefore set to 0 . The estimation of the irreducible background is done via MC simulations, where reconstructed objects are matched (in terms of $\Delta R$ ) to generator level objects, such that the contribution with fake leptons from these samples can be subtracted. This is done in order to avoid double counting of such backgrounds, because the fake background is estimated from data; it is also the reason why some numbers differ from the expectations as shown in Table 6.3.

In Figure 6.19, some of the most important distributions for the signal region are shown, such as the lepton $p_{T}$ distributions, the distribution of the missing transverse energy and a splitting of the signal events into different lepton flavour channels. There is no evidence for physics beyond the SM in that signal region.

\begin{tabular}{|c|c|}
\hline Process & Signal Region \\
\hline $\mathrm{ZZ}$ & $0.6_{-0.5}^{+0.5}$ \\
\hline ZWW & $0.12_{-0.12}^{+0.12}$ \\
\hline $\mathrm{ZZZ}$ & $<0.01_{-0.00}^{+0.00}$ \\
\hline Higgs & $0.26_{-0.07}^{+0.07}$ \\
\hline $\mathrm{t} \overline{\mathrm{t} Z}$ & $0.73_{-0.34}^{+0.34}$ \\
\hline tt̄WW & $<0.01_{-0.01}^{+0.01}$ \\
\hline Irreducible SM & $1.7_{-0.8}^{+0.8}$ \\
\hline Reducible SM & $0.00_{-0.00}^{+0.16}$ \\
\hline Total SM & $1.7_{-0.8}^{+0.8}$ \\
\hline Data & 2 \\
\hline$p_{0}$ & 0.29 \\
\hline
\end{tabular}

Table 6.6: Results in the signal region. The observed number of events is in good agreement with the expected number of events from the SM. For the estimation of the irreducible background, MC has been used, where reconstructed objects have been matched to generator level objects in order to remove events where only three of the prompt leptons, and an additional fake lepton, were reconstructed. These contributions are estimated with the weighting method. 


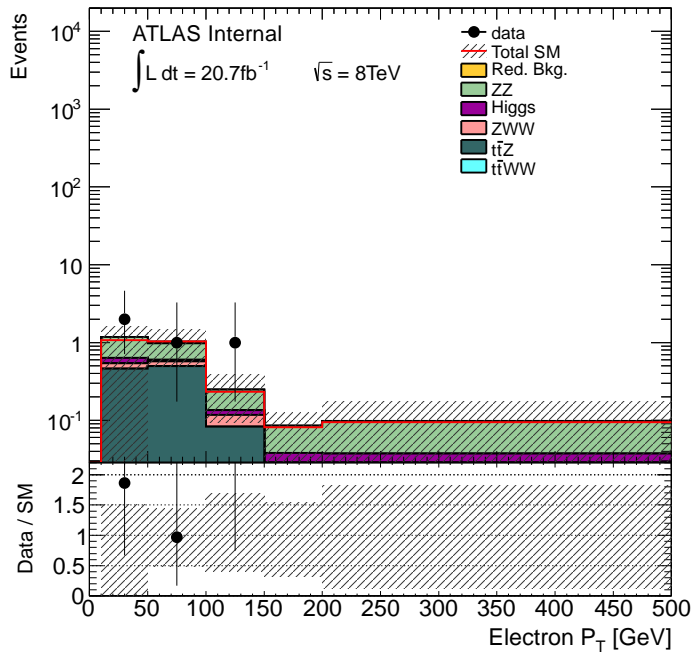

(a)

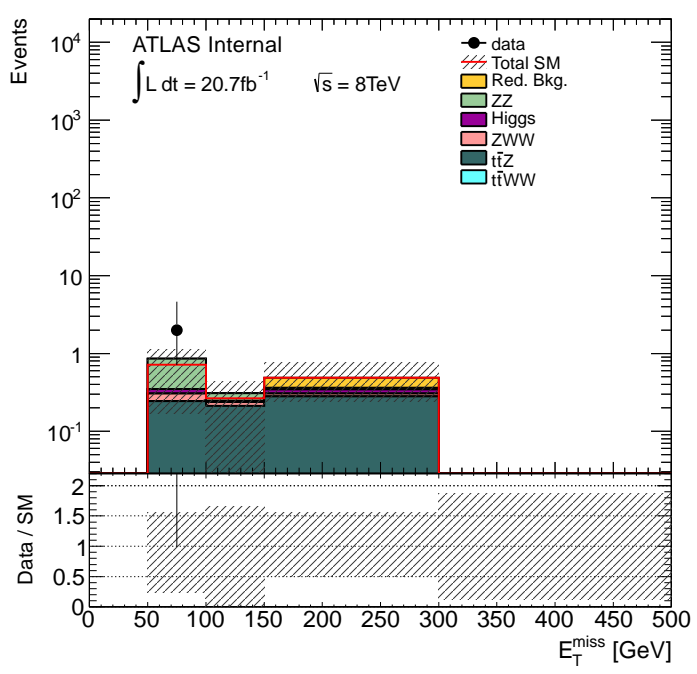

(c)

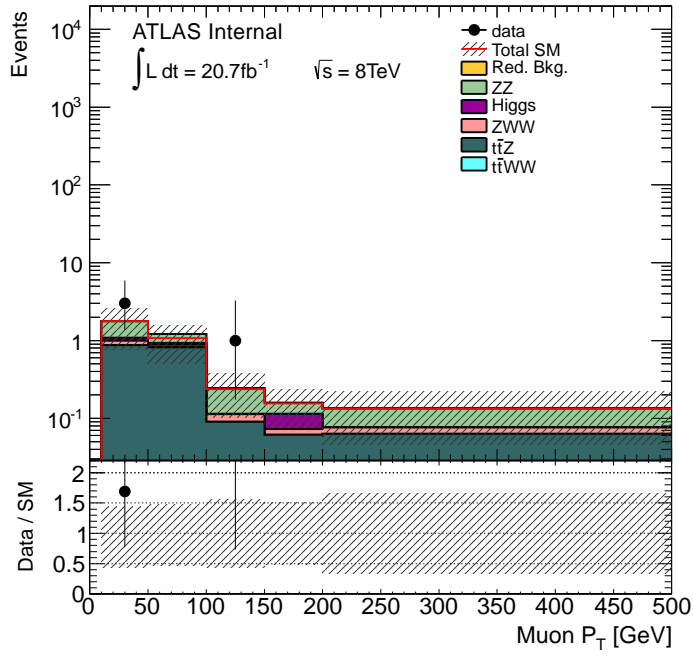

(b)

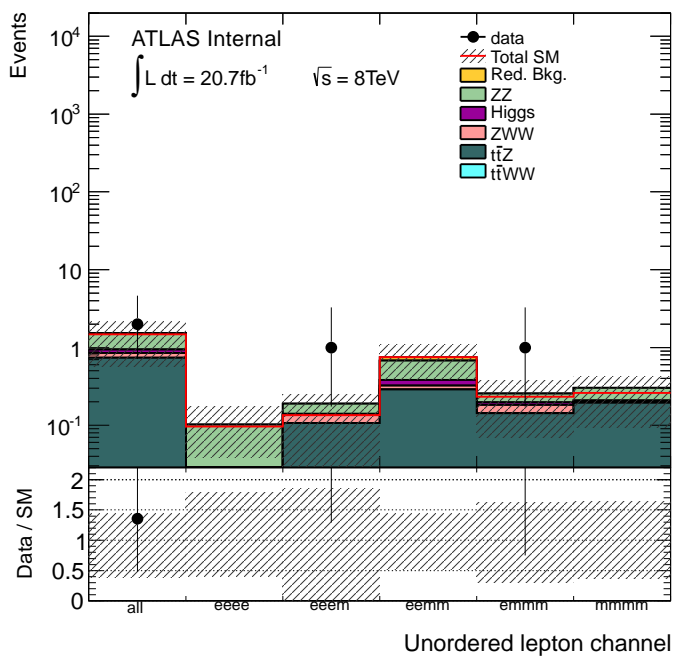

(d)

Figure 6.19: Distributions in the signal region. The agreement between the SM expectation and the observed data is reasonable [147].

\subsubsection{Statistical Interpretation of the Results}

The results of the analysis are interpreted using a profile log likelihood ratio test. The likelihood is defined using the expected number of background events and the expected number of signal events for a point in parameter space. Systematic uncertainties are included by adding nuisance parameters, where correlations between different samples are taken into account. In the case of asymmetric uncertainties, these are symmetrised for the calculation of the log likelihood ratio.

Let $n_{S}$ be the number of observed events in the signal region, $\mathbf{b}$ the background expectation, $\boldsymbol{\theta}$ the nuisance parameters for the systematic uncertainties and $\mu$ the signal strength, which is used to scale the expected number of signal events in a provided signal grid. The likelihood is 
then given by

$$
L\left(n_{S} \mid \mu, \boldsymbol{b}, \boldsymbol{\theta}\right)=P\left(n_{S} \mid \lambda_{S}(\mu, \boldsymbol{b}, \boldsymbol{\theta})\right) \cdot P_{\mathrm{syst}}\left(\boldsymbol{\theta}^{\mathbf{0}}, \boldsymbol{\theta}\right),
$$

where $P_{\text {syst }}$ is a product of unit Gaussian probability distribution functions, by which the systematic uncertainties are considered. $\lambda_{S}$ is the expected number of events in the signal region, which is a function of the expected number of background and signal events as well as the nuisance parameters $\boldsymbol{\theta}$.

The calculation of p-values and $\mathrm{CL}_{s}$ values is performed using the ATLAS HistFitter package, which is heavily based on the RooStats framework [179]. A FrequentistCalculator is used to run pseudo experiments. The number of pseudo experiments that are used varies between 30,000 and 150,000 - the calculation is done in steps of 10,000 toys and continued until the p-value is stable at the permille level for three steps.

Two different fits are considered here. First, to assess the comparability of the SM with the observed number of events in the signal region, the signal strength is set to 0 , and the probability value for the SM hypothesis is calculated. In a second step, the signal strength is set to 1 , and limits are placed in the defined simplified models for $\tilde{\chi}_{2}^{0}-\tilde{\chi}_{3}^{0}$ production.

\section{Compatibility with the Standard Model}

The SM provides a good description of the observation in the signal region. The probability to get the observed result, or one that is in worse agreement with the SM is given by $p_{0}=0.29$. There is no need to extend the SM in order to explain the observation.

\section{Limits in the Simplified Model}

For certain parameter combinations in the studied simplified models for $\tilde{\chi}_{2}^{0}-\tilde{\chi}_{3}^{0}$ production the predicted number of events (signal plus background) in the signal region is in significant disagreement with the observation. These models can therefore be excluded with a confidence level of $95 \%$, or higher.

Limits are placed in both generations of the models, and the results are shown in Figures 6.20 and 6.21. The theory uncertainty on the signal prediction is taken into account for the observed limit, while all other uncertainties are considered to calculate the expected limit and the uncertainties on the expected limit.

For a fixed mass difference $m_{\tilde{\chi}_{3}^{0}}-m_{\tilde{\chi}_{1}^{0}}=80 \mathrm{GeV}$ and $m_{\tilde{\chi}_{2}^{0}}-m_{\tilde{\chi}_{1}^{0}}=75$, Higgsino-like intermediate neutralinos with a mass of $m_{\tilde{\chi}_{3}^{0}} \lesssim 270-340 \mathrm{GeV}$ are excluded, if the masses of the first and second generation right chiral sleptons are not too close to the masses of the neutralinos.

For varying mass difference $m_{\tilde{\chi}_{3}^{0}}-m_{\tilde{\chi}_{1}^{0}}=20-80 \mathrm{GeV}$, Bino-like LSPs with a mass of $m_{\tilde{\chi}_{1}^{0}} \lesssim$ $100-250 \mathrm{GeV}$ are excluded, where for small mass differences the limit on the LSP mass gets worse. 


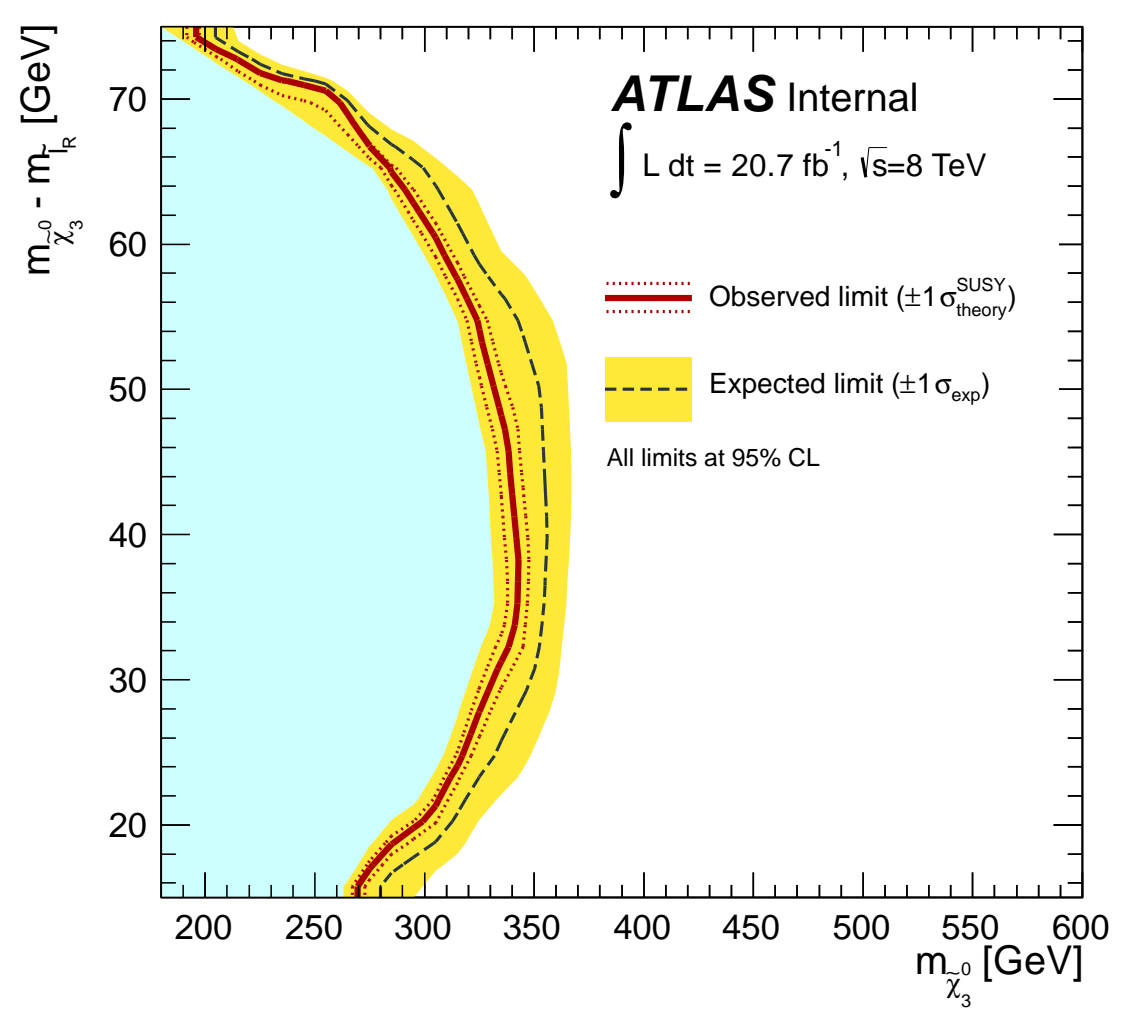

Figure 6.20: The observed and expected 95\% CL limit contours for the signal model v2A. The signal cross section uncertainty is taken into account in the observed limit. The yellow band is the $\pm 1 \sigma$ experimental uncertainty on the expected limit. The red dashed lines are the $\pm 1 \sigma$ signal theory uncertainties on the observed limit. In order to account for the discreteness of points for which $\mathrm{MC}$ has been produced, a bilinear interpolation is used to calculate the $\mathrm{CL}_{s}$ values for intermediate points. 


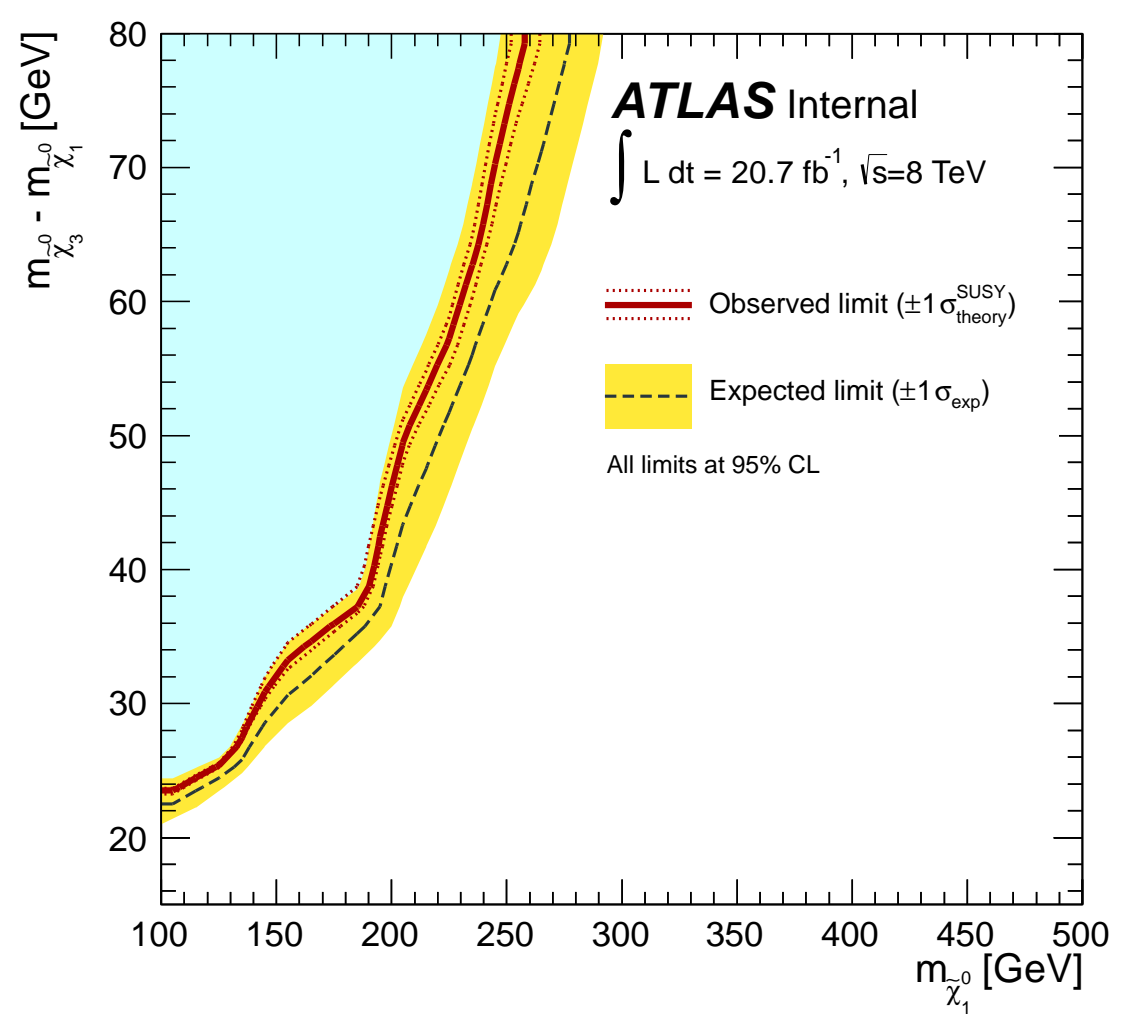

Figure 6.21: The observed and expected 95\% CL limit contours for the signal model v2B. The signal cross section uncertainty is taken into account in the observed limit. The yellow band is the $\pm 1 \sigma$ experimental uncertainty on the expected limit. The red dashed lines are the $\pm 1 \sigma$ signal theory uncertainties on the observed limit. In order to account for the discreteness of points for which $\mathrm{MC}$ has been produced, a bilinear interpolation is used to calculate the $\mathrm{CL}_{s}$ values for intermediate points. 


\section{Discussion and Outlook}

\subsection{Summary and Discussion}

A study of the CMSSM has been shown and used to motivate searches for supersymmetry in channels with a large lepton multiplicity. While the searches for SUSY at the LHC experiments ATLAS and CMS put increasingly strong constraints on constrained supersymmetric models like the CMSSM, pushing the lower boundaries on sparticle masses to larger values, a number of low energy observables prefer the superpartners of some SM particles to be light.

In the same study, the 'naturalness' of constrained models has been assessed by studying a theoretically motivated measure for the fine-tuning of a point in the CMSSM parameter space. The widely used fine-tuning measure $\Delta$ requires the superpartners to be light in order for the theory to be 'natural'. It has been shown that the constraints that were set on the CMSSM soft SUSY breaking parameters by the search for supersymmetry with jets and missing transverse energy in the final state with the ATLAS detector push the sparticle masses to large values, such that upper bounds, that were previously set on the $\Delta$ parameter, are significantly violated in the preferred regions of the CMSSM parameter space. However, placing such boundaries on the $\Delta$ parameter remains questionable.

In addition, a phenomenologically motivated measure for fine-tuning was introduced and the behaviour of this measure was studied in the CMSSM and the NUHM1, where in addition to the soft SUSY breaking parameters the top mass was considered as a parameter. It was shown that the newly introduced measure exhibits a qualitatively different behaviour in the various models that have been examined, which underlines the arbitrariness in the definition of a measure for the 'naturalness' of a model. Compared to the traditional $\Delta$ parameter, which provides a measure for the naturalness of a model by means of the $Z$ mass, the introduced parameter $\varrho_{\text {max }}$ takes into account the relations between all available measurements and the configuration of the soft SUSY breaking parameters.

As a supporting study for searches for supersymmetry in events with one or more leptons in the final state, a strategy to account for the limited efficiency of the ATLAS lepton triggers has been described. A method for the propagation of uncertainties to the sum of trigger weights, taking into account the correlation between each pair of two event weights, has been developed.

It has been shown that ATLAS trigger efficiencies measured from a data sample that is dominated by a specific physics process can be used to reweight Monte Carlo samples for any physics process, if the measured efficiencies are properly parametrised. The reweighting approach has been compared to the use of scale factors, and the advantages and disadvantages of the two methods were compared. For events with two or more leptons, it has been shown that the reweighting approach has no disadvantages compared to the scale factor approach; on the other hand, the use of scale factors has a number of shortcomings that can be avoided with the reweighting approach.

In particular the propagation of uncertainties on event yields has been studied. A method for the propagation of uncertainties on event weights to the sum of such weights has been developed, which accounts for the correlation between each pair of two events. The approximations that are made for the application of this method have been shown to be reasonable, and the estimator for 
the uncertainty on the sum of trigger weights has been shown to reflect the 'true' uncertainty very well; the developed method outperforms commonly used methods, which assume the correlation between two event weights to be $100 \%$, and can easily overestimate the 'true' uncertainty by one or more orders of magnitude. The technical implementation of that method in an existing sofware package was also shortly described.

Finally, a search for SUSY in events with four or more leptons in the final state has been performed.

A study of the pMSSM with heavy coloured sparticles and light neutralinos, charginos and sleptons has been performed; that study was then used to motivate the construction and the parametrisation of two simplified models for the direct production of a $\tilde{\chi}_{2}^{0}-\tilde{\chi}_{3}^{0}$ pair. It was shown that this production mechanism is of particular interest at the LHC if the two intermediate neutralinos are Higgsino-like; in that case, a search for SUSY in events with four or more leptons can possibly drive a discovery, if the left chiral sleptons are heavier than the lightest chargino.

The search was performed using the full ATLAS dataset of 2012, and no significant deviation from the SM prediction has been found. Limits have been set in the two simplified models, where for right chiral sleptons with a mass half-way in between the mass of the $\tilde{\chi}_{2}^{0}$ and the mass of the $\tilde{\chi}_{1}^{0}$, Higgsino-like (nearly) mass degenerate $\tilde{\chi}_{2}^{0} / \tilde{\chi}_{3}^{0}$ with a mass of less than $\sim 340 \mathrm{GeV}$, and a Bino-like neutralino LSP with a mass of less than $\sim 250 \mathrm{GeV}$ are excluded. If the masses of the right chiral sleptons are closer to the mass of either the $\tilde{\chi}_{2}^{0}$ or the $\tilde{\chi}_{1}^{0}$, the lower limits on the neutralino masses become smaller.

\subsection{Outlook}

Thanks to the sound performance of the LHC and the associated experiments, searches for physics beyond the Standard Model have been performed in many different final states - and global fits of SUSY models so far have only been using a small part of all the availabl results. For the next two years, the LHC will be on a scheduled shutdown, which means that no new data will be recorded. One of the major tasks will be the exploration of methods that can be used to implement the results in their full variety in such global fits; in particular, the SUSY fitting collaborations will work on fits of models that are less constrained than the CMSSM and the NUHMs. This will introduce additional parameters in the fits and will significantly increase both the CPU time that is needed to perform the fits, as well as the needed granularity in the signal grids that are considered for the inclusion of the LHC limits.

For instance, a fit of the pMSSM, even with only 19 parameters, has to deal with a huge parameter space; while theory calculations, like the prediction of the mass spectrum, are not necessarily more time consuming in these models than in the CMSSM, the number of points that have to be scanned by a fit in order to ensure a reliable description of the region around the minimum, grows exponentially with the number of parameters. Also, a robust parametrisation of an LHC limit will be a challenging task in such a large parameter space.

Limits are often set by the ATLAS and CMS collaborations in simplified models, as it was done in this thesis for the direct production of two neutralinos. The inclusion of such limits might as well prove to be interesting for global fits - for instance, the limits that were set in the simplified model $v 2 A$ in this document can be directly transferred to the pMSSM.

On the same page, the inclusion of LHC limits in global fits could possibly be enhanced if the experiments considered new ways of making their results public. As an example, RooStats workspaces including the full likelihood function would be very helpful in the emulation of LHC searches and the re-interpretation of the search results as a $\chi^{2}$ contribution in a global fit. 
The analysis of the data taken at the LHC with a centre-of-mass energy of $7 \mathrm{TeV}$ and $8 \mathrm{TeV}$ in the years 2010-2012 have so far not shown any hints on the existence of SUSY, or physics beyond the SM in general, although the work on some analyses still has to be concluded. Also, the methods that were used for the first analyses of that data can be refined, and a thorough evaluation of the results will be performed to be prepared for the next period of data-taking, which will presumably start in 2015.

In addition to the preparation of the analyses of the new LHC data, new measurements from the field of astroparticle physics are expected in the next years, which may provide new hints on the nature of dark matter.

It will as well be an interesting task to study the implications of a SM-like Higgs boson with a mass of $126 \mathrm{GeV}$ on SUSY. The increasing precision that is achieved in the measurements of the couplings of the Higgs-like boson will help to constrain models for physics beyond the SM even further and provides an important input to the design of searches for new physics in the next LHC run.

With the discovery of the new boson in the Higgs searches before the first long term shutdown, a big milestone at the LHC has been reached. In the next two years the road to the next milestones will be prepared, while the particle physics community will eagerly wait for the LHC to start operating again; then, at a centre-of-mass energy of $14 \mathrm{TeV}$, allowing us once again to "explore strange new worlds, discover new particles and new interactions, to boldly go where no man has gone before". 



\section{Bibliography}

[1] M. Planck, Wissenschaftliche Selbstbiographie: Mit einem Bildnis und der von Max von Laue gehaltenen Traueransprache, 2. Auflage. Johann Ambrosius Barth Verlag Leipzig, 1948.

[2] J. J. Thomson, Cathode rays, Phil. Mag. 44 (1897) 293-316.

[3] CDF Collaboration Collaboration, F. Abe et al., Observation of Top Quark Production in $\bar{p} p$ Collisions with the Collider Detector at Fermilab,

Phys. Rev. Lett. 74 (1995) 2626-2631.

http://link.aps.org/doi/10.1103/PhysRevLett.74.2626.

[4] D0 Collaboration Collaboration, S. Abachi et al., Search for high mass top quark production in $p \bar{p}$ collisions at $\sqrt{s}=1.8 \mathrm{TeV}$, Phys.Rev.Lett. 74 (1995) 2422-2426, arXiv:hep-ex/9411001 [hep-ex].

[5] DONuT Collaboration, K. Kodama et al., Final tau-neutrino results from the DONuT experiment, Phys. Rev. D78 (2008) 052002, arXiv:0711.0728 [hep-ex].

[6] ATLAS Collaboration, Observation of a new particle in the search for the Standard Model Higgs boson with the ATLAS detector at the LHC, Phys.Lett. B716 (2012) 1-29, arXiv:1207.7214 [hep-ex].

[7] CMS Collaboration, Observation of a new boson at a mass of $125 \mathrm{GeV}$ with the CMS experiment at the LHC, Phys.Lett. B716 (2012) 30-61, arXiv:1207.7235 [hep-ex].

[8] S. P. Martin, A Supersymmetry Primer, arXiv:hep-ph/9709356.

[9] G. Quast for the Electroweak Working Group, Global fit to electroweak precision data, Eur. Phys. J. C33 (2004) s641-s643.

[10] J. Haller for the Gfitter Collaboration, Fits of the Electroweak Standard Model and Beyond using Gfitter, arXiv:0810.3664 [hep-ph].

[11] H. Flacher et al., Gfitter - Revisiting the Global Electroweak Fit of the Standard Model and Beyond, Eur. Phys. J. C60 (2009) 543-583, arXiv:0811.0009 [hep-ph].

[12] A. Hoecker for the Gfitter Collaboration, Status of the global electroweak fit of the Standard Model, arXiv:0909.0961 [hep-ph].

[13] S. Weinberg, The Quantum theory of fields. Vol. 1: Foundations. Cambridge, UK: Univ. Pr. (1995) 609 p.

[14] S. Weinberg, The quantum theory of fields. Vol. 2: Modern applications. Cambridge, UK: Univ. Pr. (1996) 489 p.

[15] S. Weinberg, The quantum theory of fields. Vol. 3: Supersymmetry. Cambridge, UK: Univ. Pr. (2000) 419 p. 
[16] F. Halzen and A. Martin, Quarks and Leptons: An Introductory Course in Modern Particle Physics. John Wiley \& Sons, 1984.

[17] D. Griffiths, Introduction to Elementary Particles. Wiley-VCH Verlag GmbH \& Co. KGaA, 2004.

[18] I. J. R. Aitchison, Supersymmetry in particle physics: An elementary introduction,. SLAC-R-865.

[19] E. Noether, Invariante Variationsprobleme, Goett. Nachr. (1918) 235-257.

[20] E. P. Wigner, Gruppentheorie und ihre Anwendung auf die Quantenmechanik der Atomspektren. Vieweg, 1931.

[21] P. W. Higgs, Broken Symmetries and the Masses of Gauge Bosons, Phys. Rev. Lett. 13 (1964) 508-509.

[22] P. W. Higgs, Broken symmetries, massless particles and gauge fields, Phys.Lett. 12 (1964) 132-133.

[23] G. Guralnik, C. Hagen, and T. Kibble, Global Conservation Laws and Massless Particles, Phys. Rev. Lett. 13 (1964) 585-587.

[24] F. Englert and R. Brout, Broken Symmetry and the Mass of Gauge Vector Mesons, Phys. Rev. Lett. 13 (1964) 321-323.

[25] J. Goldstone, A. Salam, and S. Weinberg, Broken Symmetries, Phys. Rev. 127 (1962) 965-970. http://link.aps.org/doi/10.1103/PhysRev.127.965.

[26] J. Goldstone, Field Theories with Superconductor Solutions, Nuovo Cim. 19 (1961) 154-164.

[27] Y. Nambu, Quasi-Particles and Gauge Invariance in the Theory of Superconductivity, Phys. Rev. 117 (1960) 648-663. http://link.aps.org/doi/10.1103/PhysRev.117.648.

[28] LEPS Collaboration, Evidence for a narrow $S=+1$ baryon resonance in photoproduction from the neutron, Phys. Rev. Lett. 91 (2003) 012002, arXiv:hep-ex/0301020 [hep-ex].

[29] DIANA Collaboration, Observation of a baryon resonance with positive strangeness in K+ collisions with Xe nuclei, Phys.Atom.Nucl. 66 (2003) 1715-1718, arXiv:hep-ex/0304040 [hep-ex].

[30] CLAS Collaboration, A Bayesian analysis of pentaquark signals from CLAS data, Phys. Rev. Lett. 100 (2008) 052001, arXiv:0709.3154 [hep-ph].

[31] F. Close, Vanishing pentaquarks, Nature 435 (2005) 287-288.

[32] V. Kuznetsov and M. Polyakov, New Narrow Nucleon $N^{*}(1685)$, JETP Lett. 88 (2008) 347-350, arXiv:0807.3217 [hep-ph]. 
[33] CLAS Collaboration, Upper limits for the photoproduction cross section for the $\Phi^{--}(1860)$ pentaquark state off the deuteron, Phys. Rev. C85 (2012) 015205, arXiv:1109.1238 [nucl-ex].

[34] Particle Data Group, Review of Particle Physics, 2012-2013. Review of Particle Properties, Phys. Rev. D 86 no. 1, (2012) 010001.

[35] P. M. Nadolsky et al., Implications of CTEQ global analysis for collider observables, Phys. Rev. D78 (2008) 013004, arXiv:0802.0007 [hep-ph].

[36] A. Martin, W. Stirling, R. Thorne, and G. Watt, Parton distributions for the LHC, Eur. Phys. J. C63 (2009) 189-285, arXiv:0901.0002 [hep-ph].

[37] C. L. Bennett et al., 4-Year COBE DMR Cosmic Microwave Background Observations: Maps and Basic Results, Astrophys. J. 464 (1996) L1-L4, arXiv:astro-ph/9601067.

[38] D. Larson, J. Dunkley, G. Hinshaw, E. Komatsu, M. Nolta, et al., Seven-Year Wilkinson Microwave Anisotropy Probe (WMAP) Observations: Power Spectra and WMAP-Derived Parameters, Astrophys. J. Suppl. 192 (2011) 16, arXiv:1001.4635 [astro-ph.Co].

[39] N. Jarosik, C. Bennett, J. Dunkley, B. Gold, M. Greason, et al., Seven-Year Wilkinson Microwave Anisotropy Probe (WMAP) Observations: Sky Maps, Systematic Errors, and Basic Results, Astrophys. J. Suppl. 192 (2011) 14, arXiv:1001.4744 [astro-ph.Co].

[40] H. Murayama, Supersymmetry phenomenology, arXiv:hep-ph/0002232.

[41] J. Bagnasco, M. Dine, and S. D. Thomas, Detecting technibaryon dark matter, Phys.Lett. B320 (1994) 99-104, arXiv:hep-ph/9310290 [hep-ph].

[42] M. Quiros, Introduction to extra dimensions, arXiv:hep-ph/0606153 [hep-ph].

[43] C. Macesanu, The Phenomenology of universal extra dimensions at hadron colliders, Int. J. Mod. Phys. A21 (2006) 2259-2296, arXiv:hep-ph/0510418 [hep-ph].

[44] N. Arkani-Hamed, S. Dimopoulos, and G. Dvali, The Hierarchy problem and new dimensions at a millimeter, Phys.Lett. B429 (1998) 263-272, arXiv:hep-ph/9803315 [hep-ph].

[45] J. Wess and B. Zumino, Supergauge Transformations in Four-Dimensions, Nucl. Phys. B70 (1974) 39-50.

[46] S. R. Coleman and J. Mandula, ALL POSSIBLE SYMMETRIES OF THE S MATRIX, Phys. Rev. 159 (1967) 1251-1256.

[47] R. Haag, J. T. Lopuszanski, and M. Sohnius, All Possible Generators of Supersymmetries of the s Matrix, Nucl. Phys. B88 (1975) 257.

[48] J. Rosiek, Complete set of Feynman rules for the MSSM: Erratum, arXiv:hep-ph/9511250 [hep-ph].

[49] P. Gondolo and G. Gelmini, Cosmic abundances of stable particles: Improved analysis, Nucl. Phys. B360 (1991) 145-179.

[50] J. Edsjo and P. Gondolo, Neutralino relic density including coannihilations, Phys. Rev. D56 (1997) 1879-1894, arXiv:hep-ph/9704361 [hep-ph]. 
[51] G. Jungman, M. Kamionkowski, and K. Griest, Supersymmetric dark matter, Phys. Rept. 267 (1996) 195-373, arXiv:hep-ph/9506380.

[52] C. Arina and N. Fornengo, Sneutrino cold dark matter, a new analysis: Relic abundance and detection rates, JHEP 0711 (2007) 029, arXiv:0709.4477 [hep-ph].

[53] D. G. Cerdeno and A. M. Green, Direct detection of WIMPs, arXiv:1002.1912 [astro-ph.CO].

[54] DAMA Collaboration, The DAMA/LIBRA apparatus, Nucl. Instrum.Meth. A592 (2008) 297-315, arXiv:0804.2738 [astro-ph].

[55] C. Aalseth et al., Search for an Annual Modulation in a P-type Point Contact Germanium Dark Matter Detector, Phys. Rev. Lett. 107 (2011) 141301, arXiv:1106.0650 [astro-ph.CO].

[56] G. Angloher et al., Results from $730 \mathrm{~kg}$ days of the CRESST-II Dark Matter Search, Eur. Phys. J. C72 (2012) 1971, arXiv:1109.0702 [astro-ph.Co].

[57] The DARWIN Consortium, DARWIN: dark matter WIMP search with noble liquids, J. Phys. Conf. Ser. 375 (2012) 012028, arXiv:1201.2402 [astro-ph.IM].

[58] XENON100 Collaboration, E. Aprile et al., Dark Matter Results from 100 Live Days of XENON100 Data, Phys. Rev. Lett. 107 (2011) 131302, arXiv:1104.2549 [astro-ph.CO].

[59] J. R. Ellis, K. Enqvist, D. V. Nanopoulos, and F. Zwirner, Aspects of the Superunification of Strong, Electroweak and Gravitational Interactions, Nucl. Phys. B276 (1986) 14.

[60] R. Barbieri and G. Giudice, Upper Bounds on Supersymmetric Particle Masses, Nucl. Phys. B306 (1988) 63.

[61] L. Evans and P. Bryant, LHC Machine, JINST 3 (2008) S08001.

[62] http://public.web.cern.ch.

[63] ATLAS Collaboration, The ATLAS Experiment at the CERN Large Hadron Collider, JINST 3 (2008) S08003.

[64] CMS Collaboration, The CMS experiment at the CERN LHC, JINST 3 (2008) S08004.

[65] W. R. Leo, Techniques for Nuclear and Particle Physics Experiments. Springer, 2nd edition, 1994.

[66] K. Kleinknecht, Detektoren fuer Teilchenstrahlung. Teubner, 4. Auflage, 2005.

[67] LHCb Collaboration, The LHCb Detector at the LHC, JINST 3 (2008) S08005.

[68] ALICE Collaboration, K. Aamodt et al., The ALICE experiment at the CERN LHC, JINST 3 (2008) S08002.

[69] http://www.interactions.org, Image Bank.

[70] LHCf Collaboration, O. Adriani et al., The LHCf detector at the CERN Large Hadron Collider, JINST 3 (2008) S08006. 
[71] G. Antchev et al., The TOTEM detector at LHC, Nucl. Instrum.Meth. A617 (2010) 62-66.

[72] J. Pinfold et al., Technical Design Report of the MoEDAL Experiment, CERN, Geneva, 2009.

[73] ATLAS Collaboration, Updated Luminosity Determination in pp Collisions at sqrts $=7$ TeV Using the ATLAS Detector,.

[74] ATLAS Collaboration, Luminosity Determination in pp Collisions at sqrts $=7 \mathrm{TeV}$ Using the ATLAS Detector in 2011,.

[75] ATLAS Collaboration, ATLAS inner detector: Technical design report. Vol. 1, 1997.

[76] ATLAS Collaboration, ATLAS inner detector: Technical design report. Vol. 2, 1997.

[77] http://cdsweb.cern.ch.

[78] ATLAS Collaboration, ATLAS liquid argon calorimeter: Technical design report, 1996.

[79] ATLAS Collaboration, ATLAS tile calorimeter: Technical design report, 1996.

[80] ATLAS Collaboration, ATLAS muon spectrometer: Technical design report, 1997.

[81] http://atlas.web.cern.ch.

[82] ATLAS Collaboration, Electron performance measurements with the ATLAS detector using the 2010 LHC proton-proton collision data, Eur. Phys. J. C72 (2012) 1909, arXiv:1110.3174 [hep-ex].

[83] F. Teischinger and N. Lorenzo Martinez, Electron energy response stability with pile-up in 2012 data, ATL-COM-PHYS-2012-782, CERN, Geneva, 2012.

[84] ATLAS Collaboration, Calibrating the b-Tag Efficiency and Mistag Rate in $35 p b^{-1}$ of Data with the ATLAS Detector, ATLAS-CONF-2011-089, CERN, Geneva, 2011.

[85] ATLAS Collaboration, Commissioning of the ATLAS high-performance b-tagging algorithms in the $7 \mathrm{TeV}$ collision data, ATLAS-CONF-2011-102, CERN, Geneva, 2011.

[86] ATLAS Collaboration, Measurement of the b-tag Efficiency in a Sample of Jets Containing Muons with $5 \mathrm{fb}-1$ of Data from the ATLAS Detector, ATLAS-CONF-2012-043, CERN, Geneva, 2012.

[87] O. Kortner, E. Moyse, and M. Vanadia, Plots of Muon Performance in 2012 Data, ATL-COM-PHYS-2012-716, CERN, Geneva, 2012.

[88] P. Bechtle, K. Desch, and P. Wienemann, Fittino, a program for determining MSSM parameters from collider observables using an iterative method, Comput. Phys. Commun. 174 (2006) 47-70, arXiv:hep-ph/0412012.

[89] P. Bechtle, K. Desch, and P. Wienemann, Supersymmetry parameter analysis with Fittino, arXiv:hep-ph/0506244.

[90] P. Bechtle, K. Desch, and P. Wienemann, SUSY parameter measurements with Fittino, arXiv:hep-ph/0511137 [hep-ph]. 
[91] P. Bechtle, K. Desch, M. Uhlenbrock, and P. Wienemann, Constraining SUSY models with Fittino using measurements before, with and beyond the $\mathrm{LHC}$, arXiv:0907.2589 [hep-ph].

[92] P. Bechtle, K. Desch, M. Uhlenbrock, and P. Wienemann, Extracting SUSY parameters from LHC measurements using Fittino, AIP Conf.Proc. 1200 (2010) 486-489, arXiv:0909.1820 [hep-ph].

[93] P. Bechtle, K. Desch, H. Dreiner, M. Kramer, B. O'Leary, et al., Present and Possible Future Implications for mSUGRA of the Non-Discovery of SUSY at the LHC, arXiv:1105.5398 [hep-ph].

[94] P. Bechtle et al., Constrained Supersymmetry after two years of LHC data: a global view with Fittino, JHEP 1206 (2012) 098, arXiv:1204.4199 [hep-ph].

[95] W. Porod, SPheno, a program for calculating supersymmetric spectra, SUSY particle decays and SUSY particle production at $e+e$ - colliders, Comput. Phys. Commun. 153 (2003) 275-315, arXiv:hep-ph/0301101.

[96] B. C. Allanach, SOFTSUSY: A C++ program for calculating supersymmetric spectra, Comput. Phys. Commun. 143 (2002) 305-331, arXiv:hep-ph/0104145.

[97] J. A. Aguilar-Saavedra et al., Supersymmetry parameter analysis: SPA convention and project, Eur. Phys. J. C46 (2006) 43-60, arXiv:hep-ph/0511344 [hep-ph].

[98] G. Belanger, F. Boudjema, A. Pukhov, and A. Semenov, micrOMEGAs2.0: A program to calculate the relic density of dark matter in a generic model, Comput. Phys. Commun. 176 (2007) 367-382, arXiv:hep-ph/0607059.

[99] G. Belanger, F. Boudjema, A. Pukhov, and A. Semenov, Dark matter direct detection rate in a generic model with micrOMEGAs 2.2, Comput.Phys.Commun. 180 (2009) 747-767, arXiv:0803.2360 [hep-ph].

[100] T. Hahn, S. Heinemeyer, W. Hollik, H. Rzehak, and G. Weiglein, FeynHiggs 2.7, Nucl. Phys.Proc.Suppl. 205-206 (2010) 152-157, arXiv:1007.0956 [hep-ph].

[101] F. Mahmoudi, SuperIso program and flavor data constraints, PoS cHarged 2008 (2008) 020, arXiv:0812.2902 [hep-ph].

[102] N. Nguyen, D. Horns, and T. Bringmann, AstroFit: An Interface Program for Exploring Complementarity in Dark Matter Research, arXiv:1202.1385 [astro-ph.HE].

[103] A. Djouadi, J. Kalinowski, and M. Spira, HDECAY: A Program for Higgs boson decays in the standard model and its supersymmetric extension, Comput.Phys.Commun. 108 (1998) 56-74, arXiv:hep-ph/9704448 [hep-ph].

[104] Fittino Collaboration, Paper in Preparation.

[105] P. Bechtle et al., What if the LHC does not find supersymmetry in the $\sqrt{s}=7$ TeV run?, Phys. Rev. D84 (2011) 011701, arXiv:1102.4693 [hep-ph].

[106] M. Davier, A. Hoecker, B. Malaescu, and Z. Zhang, Reevaluation of the Hadronic Contributions to the Muon g-2 and to alpha(MZ)., Eur. Phys. J. C 71 (2010) 1515. 13 p. 
[107] Particle Data Group, Review of particle physics, J.Phys. G37 (2010) 075021.

[108] LHCb Collaboration Collaboration, R. Aaij et al., First evidence for the decay $B_{s} \rightarrow \mu^{+} \mu^{-}$, Phys.Rev.Lett. 110 (2013) 021801, arXiv:1211.2674 [hep-ex].

[109] Heavy Flavor Averaging Group, Averages of b-hadron, c-hadron, and $\tau$-lepton Properties, arXiv:1010.1589 [hep-ex].

[110] LHCb Collaboration, Strong constraints on the rare decays $B_{s} \rightarrow \mu^{+} \mu^{-}$and $B^{0} \rightarrow \mu^{+} \mu^{-}$, Phys. Rev. Lett. 108 (2012) 231801, arXiv:1203.4493 [hep-ex].

[111] Muon G-2 Collaboration, Final report of the muon E821 anomalous magnetic moment measurement at BNL, Phys. Rev. D73 (2006) 072003, arXiv:hep-ex/0602035.

[112] CDF Collaboration, Precise measurement of the $W$-boson mass with the CDF II detector, Phys. Rev. Lett. 108 (2012) 151803, arXiv:1203.0275 [hep-ex].

[113] D0 Collaboration, Measurement of the W Boson Mass with the D0 Detector, Phys. Rev. Lett. 108 (2012) 151804, arXiv:1203.0293 [hep-ex].

[114] The CDF and D0 Collaborations and the Tevatron Electroweak Working Group, 2012 Update of the Combination of CDF and DO Results for the Mass of the $W$ Boson, arXiv: 1204.0042 [hep-ex].

[115] The ALEPH, DELPHI, L3, OPAL and SLD Collaborations, the LEP Electroweak Working Group, and the SLD Electroweak and Heavy Flavour Groups, Precision electroweak measurements on the $Z$ resonance, Phys.Rept. 427 (2006) 257-454, arXiv:hep-ex/0509008 [hep-ex].

[116] WMAP Collaboration, E. Komatsu et al., Seven-Year Wilkinson Microwave Anisotropy Probe (WMAP) Observations: Cosmological Interpretation, Astrophys. J. Suppl. 192 (2011) 18, arXiv:1001.4538 [astro-ph.C0].

[117] ALEPH, DELPHI, L3 and OPAL Collaboration, Combined LEP Chargino Results, up to $208 \mathrm{GeV}$ for large $\mathrm{m} 0$ lepsusy.web.cern.ch/lepsusy/www/inos_moriond01/charginos_pub.html.

[118] H. K. Dreiner, S. Heinemeyer, O. Kittel, U. Langenfeld, A. M. Weber, et al., Mass Bounds on a Very Light Neutralino, Eur. Phys. J. C62 (2009) 547-572, arXiv:0901.3485 [hep-ph].

[119] J. Conley, H. Dreiner, and P. Wienemann, Measuring a Light Neutralino Mass at the ILC: Testing the MSSM Neutralino Cold Dark Matter Model, Phys. Rev. D83 (2011) 055018, arXiv:1012.1035 [hep-ph].

[120] ATLAS Collaboration, Combined search for the Standard Model Higgs boson using up to $4.9 \mathrm{fb}^{-1}$ of pp collision data at $\sqrt{s}=7 \mathrm{TeV}$ with the ATLAS detector at the LHC, Phys.Lett. B710 (2012) 49-66, arXiv:1202.1408 [hep-ex].

[121] CMS Collaboration, Combined results of searches for the standard model Higgs boson in pp collisions at sqrt(s) = 7 TeV, Phys.Lett. B710 (2012) 26-48, arXiv:1202.1488 [hep-ex]. 
[122] ATLAS Collaboration, Search for squarks and gluinos using final states with jets and missing transverse momentum with the ATLAS detector in $\sqrt{(s)}=7$ TeV proton-proton collisions, ATLAS-CONF-2012-033, CERN, Geneva, 2012.

[123] M. Bahr, S. Gieseke, M. Gigg, D. Grellscheid, K. Hamilton, et al., Herwig ++ Physics and Manual, Eur. Phys. J. C58 (2008) 639-707, arXiv:0803.0883 [hep-ph].

[124] S. Ovyn, X. Rouby, and V. Lemaitre, DELPHES, a framework for fast simulation of a generic collider experiment, arXiv:0903.2225 [hep-ph].

[125] ATLAS Collaboration, Search for squarks and gluinos using final states with jets and missing transverse momentum with the ATLAS detector in sqrts $=7$ TeV proton-proton collisions, ATLAS-CONF-2011-086, CERN, Geneva, 2011.

[126] CMS Collaboration, Search for supersymmetry with the razor variables at CMS, CMS-PAS-SUS-12-005, CERN, Geneva, 2012.

[127] P. Gondolo, J. Edsjo, P. Ullio, L. Bergstrom, M. Schelke, et al., DarkSUSY: Computing supersymmetric dark matter properties numerically, JCAP 0407 (2004) 008, arXiv:astro-ph/0406204 [astro-ph].

[128] H.E.S.S. Collaboration, F. Aharonian et al., Observations of the Crab Nebula with H.E.S.S, Astron.Astrophys. 457 (2006) 899-915, arXiv:astro-ph/0607333 [astro-ph].

[129] HESS Collaboration, A. Abramowski et al., H.E.S.S. constraints on Dark Matter annihilations towards the Sculptor and Carina Dwarf Galaxies, Astropart.Phys. 34 (2011) 608-616, arXiv:1012.5602 [astro-ph.HE].

[130] MAGIC Collaboration, J. Albert et al., VHE Gamma-Ray Observation of the Crab Nebula and Pulsar with MAGIC, Astrophys. J. 674 (2008) 1037-1055, arXiv:0705.3244 [astro-ph].

[131] T. Weekes, H. Badran, S. Biller, I. Bond, S. Bradbury, et al., VERITAS: The Very energetic radiation imaging telescope array system, Astropart.Phys. 17 (2002) 221-243, arXiv:astro-ph/0108478 [astro-ph].

[132] LAT Collaboration, W. Atwood et al., The Large Area Telescope on the Fermi Gamma-ray Space Telescope Mission, Astrophys. J. 697 (2009) 1071-1102, arXiv:0902.1089 [astro-ph.IM].

[133] M. Boezio, M. Pearce, P. Picozza, R. Sparvoli, P. Spillantini, et al., PAMELA and indirect dark matter searches, New J.Phys. 11 (2009) 105023.

[134] XENON100 Collaboration, E. Aprile et al., The XENON100 Dark Matter Experiment, Astropart.Phys. 35 (2012) 573-590, arXiv:1107.2155 [astro-ph.IM].

[135] G. W. Anderson and D. J. Castano, Measures of fine tuning, Phys.Lett. B347 (1995) 300-308, arXiv:hep-ph/9409419 [hep-ph].

[136] F. Kohn, C. Hensel, and A. Quadt, A Software Package to perform Trigger Reweighting, ATL-COM-DAQ-2010-008, CERN, Geneva, 2010. 
[137] J. Hofestädt, Muon triggers for Searches for Supersymmetry in Multi-Lepton Final States with the ATLAS Detector, Master Thesis II.Physik-UniGö-MSc-2012/05, II. Physikalisches Institut, Georg-August-Universitaet Goettingen, 2012.

[138] A. Alonso et al., Searching for Supersymmetry with two leptons and missing transverse momentum at sqrt(s)=7 TeV, ATL-PHYS-INT-2011-091, CERN, Geneva, 2011.

[139] ATLAS Collaboration, Searches for supersymmetry with the ATLAS detector using final states with two leptons and missing transverse momentum in $\sqrt{s}=7$ TeV proton-proton collisions, Phys.Lett. B709 (2012) 137-157, arXiv:1110.6189 [hep-ex].

[140] ATLAS Collaboration, Search for direct production of charginos and neutralinos in events with three leptons and missing transverse momentum in $\sqrt{s}=7$ TeV pp collisions with the ATLAS detector, arXiv:1208.3144 [hep-ex].

[141] ATLAS Collaboration, Search for direct slepton and gaugino production in final states with two leptons and missing transverse momentum with the ATLAS detector in pp collisions at $\sqrt{s}=7 \mathrm{TeV}$, arXiv:1208.2884 [hep-ex].

[142] ATLAS Collaboration, Search for supersymmetry in events with four or more leptons and missing transverse momentum in pp collisions at $\sqrt{s}=7$ TeV with the ATLAS detector, CERN, Geneva, 2012.

[143] ATLAS Collaboration, Search for R-parity-violating supersymmetry in events with four or more leptons in $\sqrt{s}=7$ TeV pp collisions with the ATLAS detector, arXiv:1210.4457 [hep-ex].

[144] ATLAS Collaboration, Expected Performance of the ATLAS Experiment - Detector, Trigger and Physics, p.266-273, arXiv:0901.0512 [hep-ex].

[145] https://twiki.cern.ch/twiki/bin/viewauth/AtlasProtected/HowToCleanJets2011, 2011.

[146] M. Cacciari et al., The Anti-k(t) jet clustering algorithm, JHEP 0804 (2008) 063, arXiv:0802.1189 [hep-ph].

[147] L. Ancu et al., Search for Supersymmetry in events with four or more leptons in 20.7 $f b^{-1}$ pp collisions at sqrt $(s)=8 \mathrm{TeV}$ with the ATLAS detector, ATL-COM-PHYS-2012-1819, CERN, Geneva, 2012.

[148] M. Mühlleitner, SDECAY: A Fortran code for SUSY particle decays in the MSSM, Acta Phys. Polon. B35 (2004) 2753-2766, arXiv:hep-ph/0409200.

[149] W. Beenakker, R. Hopker, and M. Spira, PROSPINO: A Program for the production of supersymmetric particles in next-to-leading order $Q C D$, arXiv:hep-ph/9611232 [hep-ph].

[150] L. Ancu et al., Search for supersymmetry in events with three leptons and missing transverse momentum in $13 \mathrm{fb}-1 \mathrm{pp}$ collisions at sqrt(s) $=8 \mathrm{TeV}$ with the ATLAS detector, ATL-PHYS-INT-2012-095, CERN, Geneva, 2012.

[151] L. Ancu et al., Search for Supersymmetry in events with four or more leptons in 13 fb-1 $p p$ collision at $\operatorname{sqrt}(s)=8 \mathrm{TeV}$ with the ATLAS detector, ATL-PHYS-INT-2012-096, CERN, Geneva, 2012. 
[152] https://twiki.cern.ch/twiki/bin/viewauth/AtlasProtected/ SusyObjectDefinitionsr178TeV, 2013.

[153] https://twiki.cern.ch/twiki/bin/viewauth/AtlasProtected/HowToCleanJets2012, 2012.

[154] https://twiki.cern.ch/twiki/bin/viewauth/AtlasProtected/TechnicalitiesForMedium1, 2012.

[155] https://twiki.cern.ch/twiki/bin/view/AtlasProtected/MCPAnalysisGuidelinesRel15, 2013.

[156] ATLAS Collaboration, Muon Momentum Resolution in First Pass Reconstruction of pp Collision Data Recorded by ATLAS in 2010, ATLAS-CONF-2011-046. https://cdsweb.cern.ch/record/1338575.

[157] ATLAS Collaboration, Commissioning of the ATLAS high-performance b-tagging algorithms in the $7 \mathrm{TeV}$ collision data, ATLAS-CONF-2011-102. https://cdsweb.cern.ch/record/1369219.

[158] Determination of the tau energy scale and the associated systematic uncertainty in proton-proton collisions at sqrts $==7 \mathrm{TeV}$ with the ATLAS detector at the LHC in 2011, ATLAS-CONF-2012-054, CERN, Geneva, 2012.

[159] ATLAS Collaboration, Calorimeter Clustering Algorithms: Description and Performance, ATL-LARG-PUB-2008-002. https://cdsweb. cern.ch/record/1099735.

[160] ATLAS Collaboration, Performance of the Missing Transverse Energy Reconstruction and Calibration in Proton-Proton Collisions at a Center-of-Mass Energy of $7 \mathrm{TeV}$ with the ATLAS Detector, ATLAS-CONF-2010-057. https://cdsweb. cern.ch/record/1281330.

[161] ATLAS Collaboration, ATLAS tunes of PYTHIA 6 and Pythia 8 for MC11, ATL-PHYS-PUB-2011-009. https://cdsweb. cern. ch/record/1363300.

[162] M. L. Mangano et al., ALPGEN, a generator for hard multiparton processes in hadronic collisions, JHEP 0307 (2003) 001, arXiv:hep-ph/0206293.

[163] S. Frixione, P. Nason, and C. Oleari, Matching NLO QCD computations with Parton Shower simulations: the POWHEG method, JHEP 0711 (2007) 070, arXiv:0709.2092 [hep-ph].

[164] S. Alioli, P. Nason, C. Oleari, and E. Re, A general framework for implementing NLO calculations in shower Monte Carlo programs: the POWHEG BOX, JHEP 1006 (2010) 043, arXiv:1002.2581 [hep-ph].

[165] P. Nason, A New method for combining NLO QCD with shower Monte Carlo algorithms, JHEP 0411 (2004) 040, arXiv:hep-ph/0409146 [hep-ph].

[166] J. Alwall et al., MadGraph 5 : Going Beyond, JHEP 1106 (2011) 128, arXiv:1106.0522 [hep-ph].

[167] T. Sjöstrand et al., PYTHIA 6.4 Physics and Manual, JHEP 0605 (2006) 026, arXiv:hep-ph/0603175. 
[168] S. Frixione and B. R. Webber, The MC@NLO 3.2 event generator, arXiv:hep-ph/0601192.

[169] G. Corcella, I. Knowles, G. Marchesini, S. Moretti, K. Odagiri, et al., HERWIG 6: An Event generator for hadron emission reactions with interfering gluons (including supersymmetric processes), JHEP 0101 (2001) 010, arXiv:hep-ph/0011363.

[170] J. M. Butterworth, J. R. Forshaw, and M. H. Seymour, Multiparton interactions in photoproduction at HERA, Z. Phys. C72 (1996) 637-646, arXiv:hep-ph/9601371.

[171] P. M. Nadolsky et al., Implications of CTEQ global analysis for collider observables, Phys. Rev. D78 (2008) 013004, arXiv:0802.0007 [hep-ph].

[172] H.-L. Lai et al., New parton distributions for collider physics, Phys. Rev. D82 (2010) 074024, arXiv:1007.2241 [hep-ph].

[173] T. Gleisberg et al., Event generation with SHERPA 1.1, JHEP 0902 (2009) 007, arXiv:0811.4622 [hep-ph].

[174] J. M. Campbell and R. K. Ellis, An Update on vector boson pair production at hadron colliders, Phys. Rev. D60 (1999) 113006, arXiv:hep-ph/9905386 [hep-ph].

[175] J. M. Campbell et al., Vector boson pair production at the LHC, JHEP 1107 (2011) 018, arXiv:1105.0020 [hep-ph].

[176] F. Campanario et al., QCD corrections to charged triple vector boson production with leptonic decay, Phys. Rev. D78 (2008) 094012, arXiv:0809.0790 [hep-ph].

[177] A. Kardos et al., Top quark pair production in association with a Z-boson at NLO accuracy, Phys. Rev. D85 (2012) 054015, arXiv:1111.0610 [hep-ph].

[178] J. M. Campbell and R. K. Ellis, $t \bar{t} W^{+-}$production and decay at NLO, JHEP 1207 (2012) 052, arXiv:1204.5678 [hep-ph].

[179] https://twiki.cern.ch/twiki/bin/view/RooStats/WebHome, 2012. 



\section{List of Figures}

2.1 Running of gauge couplings in the SM. Taken from [40] . . . . . . . . . . . . 14

2.2 The running of the gauge couplings in the MSSM. Taken from [40] . . . . . . . 20

2.3 An illustration of the SUSY breaking mechanism. Taken from [8]. . . . . . . . . 21

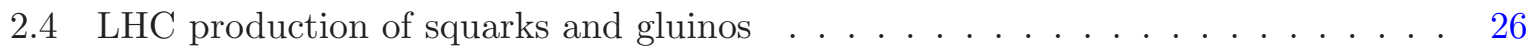

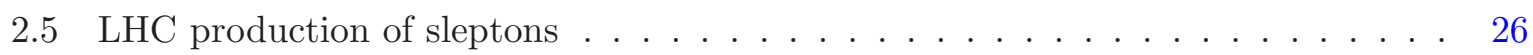

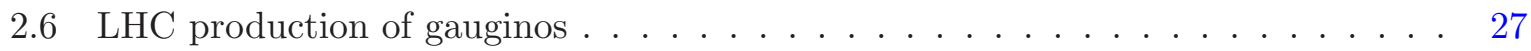

2.7 Two different diagrams contributing to the process $b \rightarrow s \gamma \ldots \ldots \ldots$

3.1 The LHC accelerator chain. Taken from $[62] \ldots \ldots \ldots$. . . . . . . . . . 34

3.2 The onion shell structure of modern HEP detectors. Taken from [69] . . . . . . . 35

3.3 Performance of the LHC and the ATLAS detector in 2011 and 2012 . . . . . 36

3.4 The ATLAS detector. Taken from $[69] \ldots \ldots \ldots$. . . . . . . . . . 37

3.5 The ATLAS inner detector. Taken from $[77] \ldots \ldots \ldots \ldots$. . . . . . . . 38

3.6 The ATLAS calorimeters. Taken from [77]. . . . . . . . . . . . . . . . 40

3.7 The ATLAS muon systems. Taken from [77]. . . . . . . . . . . . . . 42

3.8 The ATLAS trigger system. Taken from [81]. . . . . . . . . . . . . . . . 43

3.9 The ATLAS L1 muon trigger . . . . . . . . . . . . . . . . . 45

3.10 Electron E/p measurements with early data. Taken from [82] and [83] . . . . . 47

3.11 Efficiency of ATLAS $b$-tagging algorithms. Taken from $[86] \ldots \ldots$

3.12 Muon resolution and efficiency plots $[87] \ldots \ldots \ldots \ldots$

4.1 Comparison of different statistical interpretations of the same example fit based on [105]. (a) Frequentist profile likelihood; (b) Bayesian marginalisation with flat prior; (c) and (d): two different non-flat priors, as described in the Text. The black lines indicate the $2 \sigma$ (Frequentist) and 95\% CL (Bayesian) contours, respectively $[94] . \ldots \ldots \ldots \ldots \ldots \ldots \ldots \ldots$

$4.2 \chi^{2}$ contribution from the LHC SUSY search implementation compared to the published ATLAS and CMS limits $[122,126]$. Good agreement of the estimated limit with the expected limit of the LHC collaborations is achieved [94] . . . . . 57

4.3 Fittino Results $2012 \ldots \ldots$. . . . . . . . . . . . . . . 60

4.4 The minimal amount of fine-tuning as a function of $M_{0}$ and $M_{1 / 2}$, where $A_{0}$ and $\tan \beta$ are profiled [94], for (a) the pre-LHC fit and (b) the LHC fit. (c) shows the difference, $(\mathrm{b})-(\mathrm{a})$, between both fits, which is mostly close to vanishing in the overlap of the $95 \%$-CL regions. The last bin on the z-axis in (a), (b), and (c) contains all points with $\Delta>500$. The smallest values for the $\Delta$ parameter are 39.7 (193.5) for the pre-LHC (LHC) fit. . . . . . . . . . . . . 61 
4.5 Minimum value of $\varrho_{\max }$ as a function of $M_{0}$ and $M_{1 / 2}$, where $A_{0}$ and $\tan \beta$ are profiled [94]. (a) shows the minimum correlation in the 95\%-CL region of the preLHC fit, (b) shows the minimum correlation in the 95\%-CL region of the LHC fit, (c) shows the difference of both distributions. For nearly all values of $M_{0}$ and $M_{1 / 2}$, the pre-LHC fit shows a larger minimum correlation. The smallest values of $\varrho_{\max }$ are $0.62(0.29)$ for the pre-LHC (LHC) fit. . . . . . . . . . . . . . .

4.6 Minimum value of $\varrho_{\max }$ as a function of $M_{0}$ and $M_{1 / 2}$, where $A_{0}$ and $\tan \beta$ are profiled [94]. (a) and (b) show the minimum correlation for a fit of the CMSSM model with a fixed (free) $m_{t}$. For all points, the minimum correlation increases for a free $m_{t}$. The minimum value for $\left[\varrho_{\max }\right]_{\min }$ is $0.59(0.70)$ for the fit with fixed (free) $m_{t}$. In addition, (c) shows the $\varrho_{\max }$ as a function of $M_{0}$ and $M_{1 / 2}$ for a fit of the NUHM1 model, where $A_{0}, \tan \beta$ and $m_{H^{0}}$ are profiled. The minimum value of $\varrho_{\max }$ for this fit is 0.45 . All fits use $m_{h}=126 \pm 3 \mathrm{GeV}$. . . . . . . . .

4.7 The value of $\varrho_{\max }$ as a function of $M_{0}$ and $M_{1 / 2}$ at the point with the lowest $\chi^{2}$, where $A_{0}$ and $\tan \beta$ are profiled [94]. (a) shows the minimum value for a fit of the CMSSM model where $m_{t}$ is fixed, (b) shows the minimum value for a fit of the CMSSM model where $m_{t}$ is free. (c) shows the difference of the two distributions. Near the best-fit point, the minimum of $\varrho_{\max }$ is increased if $m_{t}$ is fixed, while at the boundaries of the 95\%-CL region the minimum value is decreased. Both fits are constrained by $m_{h}=126 \pm 3 \mathrm{GeV} \ldots \ldots \ldots \ldots$

5.1 Final states with leptons in the SM and in the MSSM . . . . . . . . . . 71

5.2 Example for a trigger turn-on curve . . . . . . . . . . . . . . 74

5.3 Efficiencies of EF_mu18 in $m c 10 b \ldots \ldots \ldots \ldots$. . . . . . . . . . . . . . . .

5.4 Result of the trigger re-weighting closure test on a $W \rightarrow \mu \nu$ sample . . . . . . 88

5.5 More detailed comparisons of the $p_{T}, \eta, \phi$ and $\mathbb{E}_{T}$ distributions in the $W \rightarrow \mu \nu$ sample . . . . . . . . . . . . . . . . . . 89

5.6 More detailed comparison of the $\eta$ and $\phi$ distribution, using a finer binning $\ldots \quad$. 89

5.7 Details for the pull distributions for the closure test $\ldots \ldots \ldots$. . . . . 90

5.8 More detailed comparisons of the $p_{T}, \eta, \phi$ and $\mathbb{E}_{T}$ distributions in the $W W$ sample 93

5.9 Probability distributions functions for trigger efficiencies . . . . . . . . . . . 95

5.10 Comparisons of the approximation for the correlation coefficient with the actual correlation coefficient. . . . . . . . . . . . . . . . 97

5.11 Outcome of the ensemble test using a realistic physics sample . . . . . . . . . . 99

5.12 Outcome of the ensemble test using a realistic physics sample . . . . . . . . 100

5.13 Efficiency of EF_mu18 during data taking in 2011, data taking periods A to I . . 105

5.14 Efficiency of EF_mu18_medium during data taking in 2011, periods J to M . . . . 106

5.15 Efficiency of EF_mu6 during data taking in 2011, periods A to M . . . . . . . 106

5.16 Conditional efficiencies for EF_mu10_loose . . . . . . . . . . . . . 107

6.1 Dominant vertices for direct gaugino production with a neutralino . . . . . . . 112

6.2 Relevant vertices for the decay of sleptons . . . . . . . . . . . . . . . 113

6.3 Neutralino pair production cross sections in the pMSSM . . . . . . . . . . 113

6.4 Four lepton cross section in the pMSSM . . . . . . . . . . . . . 116

6.5 The ratio $\mathcal{R}$ in the pMSSM. . . . . . . . . . . . . . . 118

6.6 Characteristics of the potentially interesting points in the pMSSM . . . . . 120

6.7 Mass spectra of some of the potentially interesting points in the pMSSM . . . . . 121 
6.8 Production cross section and estimated acceptance for the simplified models $v 2 A$

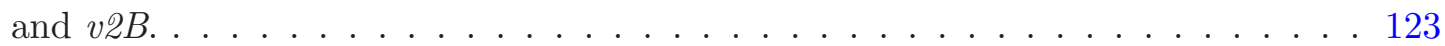

6.9 Several distributions limiting the acceptance in the simplified model v2A. . . . . 124

6.10 Estimated expected limit in the simplified model v2A . . . . . . . . . . . 125

6.11 Estimated expected limit in the simplified model v2B . . . . . . . . . . 126

6.12 Optimisation of the signal region . . . . . . . . . . . . . . 135

6.13 The selection efficiency for the signal region (top), the expected number of signal events (middle) and expected significance $Z_{N}$ (bottom) across the simplified signal models for $\tilde{\chi}_{3}^{0}-\tilde{\chi}_{2}^{0}$ production in the signal region. For the grid points where the signal is much larger than the SM background, $Z_{N}$ is set by hand to a value of 5 . Bilinear interpolation is used to calculate the values in between the discrete grid points. . . . . . . . . . . . . . . . . . 136

6.14 The total systematic uncertainty on the expected number of signal events. For most points, the signal uncertainty is roughly $10 \%-20 \%$. Points with a small acceptance and efficiency exhibit a larger uncertainty, mainly due to the small statistics after selection in the MC samples. . . . . . . . . . . . . 137

6.15 Electron Fake Ratios and Scale Factors . . . . . . . . . . . . . . . . . . . 143

6.16 Muon Fake Ratios and Scale Factors . . . . . . . . . . . . . . . . . . . . . . . 144

6.17 Validation Region VRnoZ . . . . . . . . . . . . . . . . . . 146

6.18 Validation Region VRZ . . . . . . . . . . . . . . . . . . 147

6.19 Signal Region . . . . . . . . . . . . . . . . . . . . . . . . 149

6.20 Limits in Simplified Model v2A . . . . . . . . . . . . . . . . 151

6.21 Limits in Simplified Model $v 2 B$. . . . . . . . . . . . . . . . 152 



\section{List of Tables}

2.1 The fermions in the $\mathrm{SM} \ldots \ldots \ldots \ldots \ldots \ldots \ldots$

2.2 The vector bosons in the $\mathrm{SM} \ldots \ldots \ldots \ldots \ldots$

2.3 Quantum numbers of SM fields . . . . . . . . . . . . . . . . . 11

2.4 Particle Content in the MSSM . . . . . . . . . . . . . . . . . . . 19

3.1 ATLAS data taking periods in 2011 and $2012 \ldots \ldots \ldots$. . . . . . . . . 36

4.1 Results of an example fit for the Frequentist and the Bayesian interpretation as published in [94]. For the Frequentist interpretation the point with the smallest $\chi^{2}$ is given with the corresponding 1-dimensional $1 \sigma$ uncertainties. For the Bayesian interpretation, the global mode of the full posterior pdf in the case of flat priors is given, i.e. the point with the highest local $4 \mathrm{D}$ point density. In addition the maxima of the marginalised 1-dimensional posteriors are shown with the boundaries of the smallest interval covering $68 \%$ around the maximum. . . . 53

4.2 Standard Model parameters . . . . . . . . . . . . . . . . . . 55

4.3 Low-energy observables employed. In general, experimental and theoretical uncertainties have been added in quadrature. The top quark mass $m_{t}$ is only used as an observable for the fit where $m_{t}$ is also floating as SM input parameter [94].

4.4 Summary of fit results . . . . . . . . . . . . . . . . . .

5.1 Result of the closure test using $20 \mathrm{~W} \rightarrow \mu \nu$ samples. The quoted uncertainties reflect the binomial distribution accounting the trigger decision (first term) and the statistically limited efficiency measurements (second term). The asymmetric Gaussian approximation was used to calculate the second part of the uncertainties. 91

5.2 Result of the closure test using 20 inclusive $W W$ samples requiring exactly 2 muons in the final state. Again, two sources of the uncertainties were considered, as quoted in the table. Here, the symmetric Gaussian approximation was used. On average the reweighting approach overestimates the event yield, as expected.

5.3 Outcome of the sanity checks using various samples and requiring various muon multiplicities in the final state. The overall agreement is good, however in particular in the 1 muon channels relatively large differences are observed. These are of the order of $1 \%$ and can most likely be accounted to the used binning. Note that the estimated effect of the defect can only give a very rough idea of the actual impact, which tends to increase the discrepancy in the 1 muon channels while it tends to decrease the discrepancies in the channels with more than 1 muon in the final state. . . . . . . . . . . . . . . . . . . . . 94

5.4 Results for test of quality of equation $(5.34) \ldots \ldots \ldots \ldots$

6.1 A list of potentially interesting points in the pMSSM for a four lepton search . . 119

6.2 Summary of used triggers . . . . . . . . . . . . . . . . . 128

6.3 Background expectation in signal region from $\mathrm{MC}$ only . . . . . . . . . . . 140 
6.4 Average Fake Ratios, Scale Factors and Fraction of Events in SR . . . . . . . . 142

6.5 Comparison of Expectations and Observed Data in Validation Regions . . . . . . 145

6.6 The epected and observed number of events in the signal region. . . . . . . . . 148 


\section{Acknowledgements}

First of all, I would like to thank Dr. Carsten Hensel and Prof. Dr. Arnulf Quadt for the opportunity to work in the Emmy-Noether research group at the II. Institute of Physics.

I would like to express my gratitude to Carsten in particular for his constant help and advice, and continous support. Thank you!

I would also like to thank Dr. Kevin Kröninger, who has been a great help during my work at the II. Institute.

My special thanks also go to Dr. Julien Morel, Dr. Fabian Kohn, and Dr. Alexander Mann: It was a pleasure to work with you.

I have much enjoyed my work in the Fittino Collaboration, thanks to all of you, in particular Dr. Philip Bechtle.

I am also grateful for the experiences I have made in the ATLAS SUSY Multilepton Group and the ATLAS ECAL Team - thanks to all of you.

I want to thank Dr. Philip Bechtle, Dr. Kevin Kröninger, Prof. Dr. Steffen Schumann and Dr. Jens Weingarten for proofreading significant parts of this thesis.

On the personal side, I would like to express my thankfulness to Philipp, Simon, Fabian, Martina and Elisabeth, and all the other great people I had a great time knowing during my time in Göttingen - sorry I don't list all of you by name!

Finally, I sincerely thank my parents for continuously supporting me during my time in Göttingen. Thank you, Monika and Josef! 



\section{Lebenslauf}

Lebenslauf von Matthias Hamer

$06 / 2005$

$10 / 2005-03 / 2010$

$03 / 2010$

04/2010 - heute geboren am 28.01.1986 in Paderborn als deutscher Staaatsürger

Abitur am Liebfrauen-Gymnasium Büren

Studium der Physik an der Georg-AugustUniversität Göttingen

Diplom im Fach Physik an der Goerg-AugustUniversität Göttingen mit einer Diplomarbeit zum Thema "Studies of the Inverse Problem in Supersymmetry in Particle Physics"

Doktorand am II. Physikalischen Institut der Goerg-August-Universität Göttingen 